\title{
AN EFFICIENT PREPARATION OF NEW SULFONYL FLUORIDES AND LITHIUM SULFONATES
}

Fabien Toulgoat, ${ }^{[\mathrm{a}]}$ Bernard. R. Langlois, ${ }^{[\mathrm{a}]} *$ Maurice Médebielle, ${ }^{[\mathrm{a}]} *$ Jean-Yves Sanchez ${ }^{[\mathrm{b}]}$

[a] ICBMS, Institut de Chimie et Biochimie Moléculaires et Supramoléculaires, Equipe SERCOF, 43 boulevard du 11 novembre 1918, Villeurbanne, F-69622, France ; CNRS, UMR5246, Villeurbanne, F-69622, France ; université de Lyon, Lyon, F69622, France ; université Lyon 1, Lyon, F-69622, France ; INSA-Lyon, Villeurbanne, F-69622, France ; CPE Lyon, Villeurbanne, F-69616, France.

[b] Laboratoire d'Electrochimie et de Physico-chimie des Matériaux et des Interfaces LEPMI, UMR 5631 CNRS-INPG-UJF, BP.75. 38402 Saint-Martin-d'Hères, France.

langlois@univ-lyon1.fr; medebiel@univ-lyon1.fr

\section{SUPPORTING INFORMATION}

\section{$\underline{\text { Table of Contents }}$}

\begin{tabular}{|c|c|}
\hline Information & Page \\
\hline General Experimental Methods & S4 \\
\hline Description of compounds & S5-S10 \\
\hline${ }^{1} \mathrm{H}$ NMR spectrum of $\mathbf{1 a}$ in $\mathrm{CDCl}_{3}$ at $300 \mathrm{MHz}$ & S11 \\
\hline${ }^{1} \mathrm{H}$ NMR spectrum of $\mathbf{2 a}$ in $\mathrm{CDCl}_{3}$ at $300 \mathrm{MHz}$ & S12 \\
\hline${ }^{1} \mathrm{H}$ NMR spectrum of $\mathbf{2 b}$ in $\mathrm{CDCl}_{3}$ at $300 \mathrm{MHz}$ & S13 \\
\hline${ }^{19} \mathrm{~F}$ NMR spectrum of $\mathbf{2 b}$ in $\mathrm{CDCl}_{3}$ at $282 \mathrm{MHz}$ & S14 \\
\hline${ }^{13} \mathrm{C} \mathrm{NMR}$ spectrum of $\mathbf{2 b}$ in $\mathrm{CDCl}_{3}$ at $75 \mathrm{MHz}$ & S15 \\
\hline${ }^{1} \mathrm{H}$ NMR spectrum of $\mathbf{3 a}$ in $\mathrm{CDCl}_{3}$ at $300 \mathrm{MHz}$ & S16 \\
\hline${ }^{19} \mathrm{~F} \mathrm{NMR}$ spectrum of $\mathbf{3 a}$ in $\mathrm{CDCl}_{3}$ at $282 \mathrm{MHz}$ & S17 \\
\hline${ }^{13} \mathrm{C}$ NMR spectrum of $\mathbf{3 a}$ in $\mathrm{CDCl}_{3}$ at $75 \mathrm{MHz}$ & S18 \\
\hline${ }^{1} \mathrm{H}$ NMR spectrum of $\mathbf{4 a}$ in $\mathrm{CDCl}_{3}$ at $300 \mathrm{MHz}$ & S19 \\
\hline${ }^{1} \mathrm{H}$ NMR spectrum of $\mathbf{4 b}$ in $\mathrm{CDCl}_{3}$ at $300 \mathrm{MHz}$ & S20 \\
\hline${ }^{19} \mathrm{~F} \mathrm{NMR}$ spectrum of $\mathbf{4 b}$ in $\mathrm{CDCl}_{3}$ at $282 \mathrm{MHz}$ & S21 \\
\hline${ }^{13} \mathrm{C} \mathrm{NMR}$ spectrum of $\mathbf{4 b}$ in $\mathrm{CDCl}_{3}$ at $75 \mathrm{MHz}$ & S22 \\
\hline${ }^{1} \mathrm{H}$ NMR spectrum of $\mathbf{5 a}$ in $\mathrm{CDCl}_{3}$ at $300 \mathrm{MHz}$ & S23 \\
\hline
\end{tabular}




\begin{tabular}{|c|c|}
\hline${ }^{19} \mathrm{~F}$ NMR spectrum of $\mathbf{5 a}$ in $\mathrm{CDCl}_{3}$ at $282 \mathrm{MHz}$ & S24 \\
\hline${ }^{13} \mathrm{C}$ NMR spectrum of $\mathbf{5 a}$ in $\mathrm{CDCl}_{3}$ at $75 \mathrm{MHz}$ & S25 \\
\hline${ }^{1} \mathrm{H}$ NMR spectrum of $\mathbf{5 b}$ in $\mathrm{CDCl}_{3}$ at $300 \mathrm{MHz}$ & S26 \\
\hline${ }^{19} \mathrm{~F}$ NMR spectrum of $\mathbf{5 b}$ in $\mathrm{CDCl}_{3}$ at $282 \mathrm{MHz}$ & S27 \\
\hline${ }^{13} \mathrm{C}$ NMR spectrum of $\mathbf{5 b}$ in $\mathrm{CDCl}_{3}$ at $75 \mathrm{MHz}$ & S28 \\
\hline${ }^{1} \mathrm{H}$ NMR spectrum of $\mathbf{5 c}$ in $\mathrm{CDCl}_{3}$ at $300 \mathrm{MHz}$ & S29 \\
\hline${ }^{19} \mathrm{~F}$ NMR spectrum of $\mathbf{5 c}$ in $\mathrm{CDCl}_{3}$ at $282 \mathrm{MHz}$ & S30 \\
\hline${ }^{13} \mathrm{C}$ NMR spectrum of $\mathbf{5 c}$ in $\mathrm{CDCl}_{3}$ at $75 \mathrm{MHz}$ & S31 \\
\hline${ }^{1} \mathrm{H}$ NMR spectrum of $\mathbf{5 d}$ in $\mathrm{CDCl}_{3}$ at $300 \mathrm{MHz}$ & S32 \\
\hline${ }^{19} \mathrm{~F}$ NMR spectrum of $\mathbf{5 d}$ in $\mathrm{CDCl}_{3}$ at $282 \mathrm{MHz}$ & S33 \\
\hline${ }^{13} \mathrm{C} \mathrm{NMR} \mathrm{spectrum} \mathrm{of} \mathbf{5 d}$ in $\mathrm{CDCl}_{3}$ at $75 \mathrm{MHz}$ & S34 \\
\hline${ }^{1} \mathrm{H}$ NMR spectrum of $\mathbf{6 a}$ in $\mathrm{CDCl}_{3}$ at $300 \mathrm{MHz}$ & S35 \\
\hline${ }^{19} \mathrm{~F}$ NMR spectrum of $\mathbf{6 a}$ in $\mathrm{CDCl}_{3}$ at $282 \mathrm{MHz}$ & S36 \\
\hline${ }^{13} \mathrm{C}$ NMR spectrum of $\mathbf{6 a}$ in $\mathrm{CDCl}_{3}$ at $75 \mathrm{MHz}$ & S37 \\
\hline${ }^{1} \mathrm{H}$ NMR spectrum of $\mathbf{7 a}$ in $\mathrm{CDCl}_{3}$ at $300 \mathrm{MHz}$ & S38 \\
\hline${ }^{19} \mathrm{~F}$ NMR spectrum of $\mathbf{7 a}$ in $\mathrm{CDCl}_{3}$ at $282 \mathrm{MHz}$ & S39 \\
\hline${ }^{13} \mathrm{C}$ NMR spectrum of $\mathbf{7 a}$ in $\mathrm{CDCl}_{3}$ at $75 \mathrm{MHz}$ & $\mathrm{S} 40$ \\
\hline${ }^{\mathrm{I}} \mathrm{H} \mathrm{NMR}$ spectrum of $\mathbf{8 a}$ in $\mathrm{CDCl}_{3}$ at $300 \mathrm{MHz}$ & S41 \\
\hline${ }^{19} \mathrm{~F} \mathrm{NMR} \mathrm{spectrum} \mathrm{of} \mathbf{8 a}$ in $\mathrm{CDCl}_{3}$ at $282 \mathrm{MHz}$ & $\mathrm{S} 42$ \\
\hline${ }^{13} \mathrm{C} \mathrm{NMR} \mathrm{spectrum} \mathrm{of} \mathbf{8 a}$ in $\mathrm{CDCl}_{3}$ at $75 \mathrm{MHz}$ & $\mathrm{S} 43$ \\
\hline${ }^{1} \mathrm{H}$ NMR spectrum of 9 in $\mathrm{CDCl}_{3}$ at $300 \mathrm{MHz}$ & S44 \\
\hline${ }^{1} \mathrm{H}$ NMR spectrum of 11 in $\mathrm{CDCl}_{3}$ at $300 \mathrm{MHz}$ & $\mathrm{S} 45$ \\
\hline${ }^{19} \mathrm{~F}$ NMR spectrum of 11 in $\mathrm{CDCl}_{3}$ at $282 \mathrm{MHz}$ & S46 \\
\hline${ }^{13} \mathrm{C} \mathrm{NMR} \mathrm{spectrum} \mathrm{of} \mathbf{1 1}$ in $\mathrm{CDCl}_{3}$ at $75 \mathrm{MHz}$ & S47 \\
\hline${ }^{1} \mathrm{H}$ NMR spectrum of $\mathbf{1 3 a}$ in $\mathrm{CDCl}_{3}$ at $300 \mathrm{MHz}$ & $\mathrm{S} 48$ \\
\hline${ }^{19} \mathrm{~F}$ NMR spectrum of $\mathbf{1 3 a}$ in $\mathrm{CDCl}_{3}$ at $282 \mathrm{MHz}$ & S49 \\
\hline${ }^{13} \mathrm{C}$ NMR spectrum of $13 a$ in $\mathrm{CDCl}_{3}$ at $75 \mathrm{MHz}$ & S50 \\
\hline${ }^{1} \mathrm{H}$ NMR spectrum of $\mathbf{1 3 b}$ in $\mathrm{CDCl}_{3}$ at $300 \mathrm{MHz}$ & S51 \\
\hline${ }^{19} \mathrm{~F}$ NMR spectrum of $\mathbf{1 3 b}$ in $\mathrm{CDCl}_{3}$ at $282 \mathrm{MHz}$ & S52 \\
\hline${ }^{13} \mathrm{C}$ NMR spectrum of $\mathbf{1 3 b}$ in $\mathrm{CDCl}_{3}$ at $75 \mathrm{MHz}$ & S53 \\
\hline${ }^{1} \mathrm{H}$ NMR spectrum of $\mathbf{1 3 c}$ in $\mathrm{CDCl}_{3}$ at $300 \mathrm{MHz}$ & S54 \\
\hline${ }^{19} \mathrm{~F}$ NMR spectrum of $13 \mathrm{c}$ in $\mathrm{CDCl}_{3}$ at $282 \mathrm{MHz}$ & S55 \\
\hline${ }^{13} \mathrm{C} \mathrm{NMR}$ spectrum of $13 \mathrm{c}$ in $\mathrm{CDCl}_{3}$ at $75 \mathrm{MHz}$ & S56 \\
\hline${ }^{1} \mathrm{H}$ NMR spectrum of $\mathbf{1 3 d}$ in $\mathrm{CDCl}_{3}$ at $300 \mathrm{MHz}$ & S57 \\
\hline${ }^{19} \mathrm{~F}$ NMR spectrum of $\mathbf{1 3 d}$ in $\mathrm{CDCl}_{3}$ at $282 \mathrm{MHz}$ & S58 \\
\hline${ }^{13} \mathrm{C}$ NMR spectrum of $\mathbf{1 3 d}$ in $\mathrm{CDCl}_{3}$ at $75 \mathrm{MHz}$ & S59 \\
\hline${ }^{1} \mathrm{H}$ NMR spectrum of $\mathbf{1 4 a}$ in $\mathrm{CDCl}_{3}$ at $300 \mathrm{MHz}$ & S60 \\
\hline${ }^{19} \mathrm{~F}$ NMR spectrum of $\mathbf{1 4 a}$ in $\mathrm{CDCl}_{3}$ at $282 \mathrm{MHz}$ & S61 \\
\hline${ }^{13} \mathrm{C}$ NMR spectrum of $\mathbf{1 4 a}$ in $\mathrm{CDCl}_{3}$ at $75 \mathrm{MHz}$ & S62 \\
\hline${ }^{1} \mathrm{H}$ NMR spectrum of 15 in $\mathrm{CDCl}_{3}$ at $300 \mathrm{MHz}$ & S63 \\
\hline${ }^{19} \mathrm{~F}$ NMR spectrum of 15 in $\mathrm{CDCl}_{3}$ at $282 \mathrm{MHz}$ & S64 \\
\hline${ }^{13} \mathrm{C}$ NMR spectrum of $\mathbf{1 5}$ in $\mathrm{CDCl}_{3}$ at $75 \mathrm{MHz}$ & S65 \\
\hline${ }^{1} \mathrm{H}$ NMR spectrum of $\mathbf{1 6 a}$ in $\mathrm{CDCl}_{3}$ at $300 \mathrm{MHz}$ & S66 \\
\hline${ }^{19} \mathrm{~F}$ NMR spectrum of $\mathbf{1 6 a}$ in $\mathrm{CDCl}_{3}$ at $282 \mathrm{MHz}$ & S67 \\
\hline${ }^{13} \mathrm{C}$ NMR spectrum of $\mathbf{1 6 a}$ in $\mathrm{CDCl}_{3}$ at $75 \mathrm{MHz}$ & S68 \\
\hline${ }^{1} \mathrm{H}$ NMR spectrum of $\mathbf{1 6 b}$ in $\mathrm{CDCl}_{3}$ at $300 \mathrm{MHz}$ & S69 \\
\hline${ }^{19} \mathrm{~F}$ NMR spectrum of $\mathbf{1 6} \mathbf{b}$ in $\mathrm{CDCl}_{3}$ at $282 \mathrm{MHz}$ & $\mathrm{S} 70$ \\
\hline${ }^{13} \mathrm{C}$ NMR spectrum of $\mathbf{1 6 b}$ in $\mathrm{CDCl}_{3}$ at $75 \mathrm{MHz}$ & S71 \\
\hline
\end{tabular}




\begin{tabular}{|c|c|}
\hline${ }^{1} \mathrm{H}$ NMR spectrum of $\mathbf{1 7 a}$ in $\mathrm{CDCl}_{3}$ at $300 \mathrm{MHz}$ & S72 \\
\hline${ }^{19} \mathrm{~F}$ NMR spectrum of $\mathbf{1 7 a}$ in $\mathrm{CDCl}_{3}$ at $282 \mathrm{MHz}$ & S73 \\
\hline${ }^{13} \mathrm{C}$ NMR spectrum of $\mathbf{1 7 a}$ in $\mathrm{CDCl}_{3}$ at $75 \mathrm{MHz}$ & S74 \\
\hline${ }^{1} \mathrm{H}$ NMR spectrum of $\mathbf{1 8 a}$ in $\mathrm{CDCl}_{3}$ at $300 \mathrm{MHz}$ & S75 \\
\hline${ }^{19} \mathrm{~F}$ NMR spectrum of $\mathbf{1 8 a}$ in $\mathrm{CDCl}_{3}$ at $282 \mathrm{MHz}$ & S76 \\
\hline${ }^{13} \mathrm{C}$ NMR spectrum of $\mathbf{1 8 a}$ in $\mathrm{CDCl}_{3}$ at $75 \mathrm{MHz}$ & S77 \\
\hline${ }^{1} \mathrm{H}$ NMR spectrum of $\mathbf{5 c}$ in $\mathrm{CDCl}_{3}$ at $300 \mathrm{MHz}$ & S78 \\
\hline${ }^{19} \mathrm{~F}$ NMR spectrum of $\mathbf{5 c}$ in $\mathrm{CDCl}_{3}$ at $282 \mathrm{MHz}$ & S79 \\
\hline${ }^{13} \mathrm{C} \mathrm{NMR} \mathrm{spectrum} \mathrm{of} \mathbf{5 c}$ in $\mathrm{CDCl}_{3}$ at $75 \mathrm{MHz}$ & S80 \\
\hline${ }^{1} \mathrm{H}$ NMR spectrum of $\mathbf{1 9 a}$ in $\mathrm{CDCl}_{3}$ at $300 \mathrm{MHz}$ & S81 \\
\hline${ }^{19} \mathrm{~F}$ NMR spectrum of $\mathbf{1 9 a}$ in $\mathrm{CDCl}_{3}$ at $282 \mathrm{MHz}$ & S82 \\
\hline${ }^{13} \mathrm{C}$ NMR spectrum of $19 a$ in $\mathrm{CDCl}_{3}$ at $75 \mathrm{MHz}$ & S83 \\
\hline${ }^{1} \mathrm{H}$ NMR spectrum of $\mathbf{2 0 a}$ in $\mathrm{CDCl}_{3}$ at $300 \mathrm{MHz}$ & S84 \\
\hline${ }^{19} \mathrm{~F}$ NMR spectrum of $\mathbf{2 0 a}$ in $\mathrm{CDCl}_{3}$ at $282 \mathrm{MHz}$ & S85 \\
\hline${ }^{13} \mathrm{C}$ NMR spectrum of $\mathbf{2 0 a}$ in $\mathrm{CDCl}_{3}$ at $75 \mathrm{MHz}$ & S86 \\
\hline${ }^{1} \mathrm{H}$ NMR spectrum of 21 in $\mathrm{CDCl}_{3}$ at $300 \mathrm{MHz}$ & S87 \\
\hline${ }^{19} \mathrm{~F}$ NMR spectrum of 21 in $\mathrm{CDCl}_{3}$ at $282 \mathrm{MHz}$ & S88 \\
\hline${ }^{13} \mathrm{C} \mathrm{NMR} \mathrm{spectrum} \mathrm{of} 21$ in $\mathrm{CDCl}_{3}$ at $75 \mathrm{MHz}$ & S89 \\
\hline${ }^{1} \mathrm{H}$ NMR spectrum of 22 in $\mathrm{CDCl}_{3}$ at $300 \mathrm{MHz}$ & S90 \\
\hline${ }^{19} \mathrm{~F}$ NMR spectrum of 22 in $\mathrm{CDCl}_{3}$ at $282 \mathrm{MHz}$ & S91 \\
\hline${ }^{13} \mathrm{C}$ NMR spectrum of 22 in $\mathrm{CDCl}_{3}$ at $75 \mathrm{MHz}$ & S92 \\
\hline${ }^{1} \mathrm{H}$ NMR spectrum of 23 in $\mathrm{CDCl}_{3}$ at $300 \mathrm{MHz}$ & S93 \\
\hline${ }^{19} \mathrm{~F}$ NMR spectrum of 23 in $\mathrm{CDCl}_{3}$ at $282 \mathrm{MHz}$ & S94 \\
\hline${ }^{13} \mathrm{C}$ NMR spectrum of 23 in $\mathrm{CDCl}_{3}$ at $75 \mathrm{MHz}$ & S95 \\
\hline${ }^{1} \mathrm{H}$ NMR spectrum of 24 in $\mathrm{CDCl}_{3}$ at $300 \mathrm{MHz}$ & S96 \\
\hline${ }^{19} \mathrm{~F}$ NMR spectrum of $\mathbf{2 4}$ in $\mathrm{CDCl}_{3}$ at $282 \mathrm{MHz}$ & S97 \\
\hline${ }^{13} \mathrm{C} \mathrm{NMR} \mathrm{spectrum} \mathrm{of} 24$ in $\mathrm{CDCl}_{3}$ at $75 \mathrm{MHz}$ & S98 \\
\hline${ }^{1} \mathrm{H}$ NMR spectrum of 25 in $\mathrm{CDCl}_{3}$ at $300 \mathrm{MHz}$ & S99 \\
\hline${ }^{19} \mathrm{~F} \mathrm{NMR}$ spectrum of 25 in $\mathrm{CDCl}_{3}$ at $282 \mathrm{MHz}$ & S100 \\
\hline${ }^{13} \mathrm{C}$ NMR spectrum of 25 in $\mathrm{CDCl}_{3}$ at $75 \mathrm{MHz}$ & S101 \\
\hline${ }^{1} \mathrm{H}$ NMR spectrum of 26 in $\mathrm{CDCl}_{3}$ at $300 \mathrm{MHz}$ & S102 \\
\hline${ }^{19} \mathrm{~F}$ NMR spectrum of $\mathbf{2 6}$ in $\mathrm{CDCl}_{3}$ at $282 \mathrm{MHz}$ & S103 \\
\hline${ }^{13} \mathrm{C}$ NMR spectrum of 26 in $\mathrm{CDCl}_{3}$ at $75 \mathrm{MHz}$ & S104 \\
\hline${ }^{1} \mathrm{H}$ NMR spectrum of 27 in $\mathrm{CDCl}_{3}$ at $300 \mathrm{MHz}$ & S105 \\
\hline${ }^{19} \mathrm{~F} \mathrm{NMR}$ spectrum of 27 in $\mathrm{CDCl}_{3}$ at $282 \mathrm{MHz}$ & S106 \\
\hline${ }^{13} \mathrm{C} \mathrm{NMR} \mathrm{spectrum} \mathrm{of} 27$ in $\mathrm{CDCl}_{3}$ at $75 \mathrm{MHz}$ & S107 \\
\hline
\end{tabular}




\section{$\underline{\text { General experimental methods }}$}

General. Solvents were distilled before use. Reagents were obtained commercially and used without further purification. $\mathrm{CsF}$ and $\mathrm{KF}$ were dried in an oven at $250{ }^{\circ} \mathrm{C}$ overnight. ${ }^{1} \mathrm{H},{ }^{19} \mathrm{~F}$ and ${ }^{13} \mathrm{C}$ NMR were recorded at room temperature in $\mathrm{CDCl}_{3}$ (unless stated otherwise cited) at $300 \mathrm{MHz}, 282 \mathrm{MHz}$ and $75 \mathrm{MHz}$ respectively. Chemical shifts are given in ppm relative to residual peak of solvent $\left(\delta_{\mathrm{H}}=7.26 \mathrm{ppm}\right.$ for $\mathrm{CHCl}_{3}, \delta_{\mathrm{H}}=2.50 \mathrm{ppm}$ for $\mathrm{Me}_{2} \mathrm{SO}, \delta_{\mathrm{H}}=2.05 \mathrm{ppm}$ for acetone, $\delta_{\mathrm{C}}=77.0 \mathrm{ppm}$ for $\mathrm{CDCl}_{3}, \delta_{\mathrm{C}}=39.52 \mathrm{ppm}$ for $\mathrm{Me}_{2} \mathrm{SO}-\mathrm{d}_{6}$ and $\delta_{\mathrm{C}}=29.84 \mathrm{ppm}$ for acetone- $\left.\mathrm{d}_{6}\right)$ and $\mathrm{CFCl}_{3}\left({ }^{19} \mathrm{~F}\right)$. Coupling constants are given in Hertz. TLC analyses were carried out on silica gel deposited on aluminum plates, detection being done by UV. Silica gel chromatography was performed with silica gel (230 - 400 mesh). Mass spectrometric data (MS) were obtained by electron ionization (EI, $70 \mathrm{eV}$ ), chemical ionization $\left(\mathrm{CI}, \mathrm{NH}_{3}\right.$ or $\mathrm{CH}_{4}$ ), or electrospray ionization (ESI). Melting points are uncorrected and were performed in capillary tubes. HRMS or combustion of new sulfinates $7 a$ and $8 a$ is precluded by their thermal stability. As lithium sulfonates 21-27 are hygroscopic solids, their elemental analysis were not determined. 
Synthesis of 1-(Bromo-difluoro-methylsulfanyl)-benzene (1a): ${ }^{1}$ To a suspension of sodium hydride (6 g, $150 \mathrm{mmol})$ in anhydrous DMF (100 mL), was slowly added thiophenol (10.2 $\mathrm{mL}, 100 \mathrm{mmol}$ ) at $0^{\circ} \mathrm{C}$ over a period of $30 \mathrm{~min}$. The reaction mixture was cooled to $-50^{\circ} \mathrm{C}$ for $15 \mathrm{~min}$ before bromodifluoromethane $(27 \mathrm{~mL}, 300 \mathrm{mmol})$ was added. The resulting reaction mixture was maintained at $-50^{\circ} \mathrm{C}$ for $3 \mathrm{~h}$ and stirred $1 \mathrm{~h}$ from $-50^{\circ} \mathrm{C}$ to room temperature. The resulting flask was cooled in an ice-water bath and excess sodium hydride was quenched by dropwise addition of water $(100 \mathrm{~mL})$. The aqueous phase was extracted with $\mathrm{Et}_{2} \mathrm{O}(3 \mathrm{x} 100$ $\mathrm{mL})$, the combined organic layers washed with water $(3 \times 100 \mathrm{~mL})$, brine $(100 \mathrm{~mL})$, and dried over $\mathrm{MgSO}_{4}$. Filtration and solvent evaporation left a crude product that was purified by distillation. 1a was obtained as a colorless liquid (14.3 g, 60\%). B.p. $97^{\circ} \mathrm{C} / 34 \mathrm{mmHg} ; R_{f}=0.7$ (Pentane); ${ }^{1} \mathrm{H}$ NMR: $\delta=7.66\left(\mathrm{~d}, 2 \mathrm{H},{ }^{3} \mathrm{~J}_{\mathrm{H}-\mathrm{H}}=7.4 \mathrm{~Hz}\right), 7.52(\mathrm{~m}, 1 \mathrm{H}), 7.43(\mathrm{~m}, 2 \mathrm{H}) ;{ }^{13} \mathrm{C}$ NMR: $\delta$ $=136.5,131.2,129.6,127.3\left(\mathrm{t},{ }^{3} J_{\mathrm{F}-\mathrm{C}}=1.1 \mathrm{~Hz}\right), 119.4\left(\mathrm{t},{ }^{1} J_{\mathrm{F}-\mathrm{C}}=336.0 \mathrm{~Hz}\right) ;{ }^{19} \mathrm{~F}$ NMR: $\delta=-$ $22.53(\mathrm{~s}, 2 \mathrm{~F})$.

1-(2-Bromo-1,1,2,2-tetrafluoro-ethylsulfanyl)-4-fluoro-benzene (2b): colorless liquid (83\% yield); b.p 85-90 ${ }^{\circ} \mathrm{C} / 30 \mathrm{mmHg} ; R_{f}=0.8$ (Pentane); ${ }^{1} \mathrm{H}$ NMR: $\delta=7.65(\mathrm{~m}, 2 \mathrm{H}), 7.12(\mathrm{~m}$, $2 \mathrm{H}) ;{ }^{13} \mathrm{C}$ NMR: $\delta=164.8\left(\mathrm{~d},{ }^{1} J_{\mathrm{F}-\mathrm{C}}=252.5 \mathrm{~Hz}\right), 139.7\left(\mathrm{~d},{ }^{3} J_{\mathrm{F}-\mathrm{C}}=9.3 \mathrm{~Hz}\right), 122.1\left(\mathrm{ttd},{ }^{1} J_{\mathrm{F}-\mathrm{C}}=\right.$ $\left.290.9 \mathrm{~Hz},{ }^{2} J_{\mathrm{F}-\mathrm{C}}=34.3 \mathrm{~Hz},{ }^{6} J_{\mathrm{F}-\mathrm{C}}=1.5 \mathrm{~Hz}\right), 118.9(\mathrm{~m}), 116.9\left(\mathrm{~d},{ }^{2} J_{\mathrm{F}-\mathrm{C}}=22.0 \mathrm{~Hz}\right) 116.8\left(\mathrm{tt},{ }^{1} J_{\mathrm{F}-\mathrm{C}}\right.$ $\left.=313.4 \mathrm{~Hz},{ }^{2} J_{\mathrm{F}-\mathrm{C}}=40.4 \mathrm{~Hz}\right) ;{ }^{19} \mathrm{~F}$ NMR: $\delta=-62.71\left(\mathrm{t}, 2 \mathrm{~F},{ }^{3} J_{\mathrm{F}-\mathrm{F}}=8.0 \mathrm{~Hz}\right),-108.88\left(\mathrm{t}, 2 \mathrm{~F},{ }^{3} J_{\mathrm{F}-\mathrm{F}}=\right.$ $8.0 \mathrm{~Hz}$ ) -108.88 (m, 1F). Anal. Calcd for $\mathrm{C}_{8} \mathrm{H}_{4} \mathrm{BrF}_{5} \mathrm{~S}$ : C, 31.29; H, 1.31. Found C, 31.64; H, 1.58 .

1-Bromo-4-(2-Bromo-1,1,2,2-tetrafluoro-ethylsulfanyl)-benzene (2c): colorless liquid (90\% yield); $R_{f}=0.8$ (Pentane); ${ }^{1} \mathrm{H}$ NMR: $\delta=7.57-7.49(\mathrm{~m}, 4 \mathrm{H}) ;{ }^{13} \mathrm{C} \mathrm{NMR}: \delta=138.7,132.8$, $126.4,122.6\left(\mathrm{t},{ }^{3} J_{\mathrm{F}-\mathrm{C}}=2.7 \mathrm{~Hz}\right), 121.9\left(\mathrm{tt},{ }^{1} J_{\mathrm{F}-\mathrm{C}}=291.2 \mathrm{~Hz},{ }^{2} J_{\mathrm{F}-\mathrm{C}}=34.2 \mathrm{~Hz}\right), 116.69\left(\mathrm{tt},{ }^{1} J_{\mathrm{F}-\mathrm{C}}=\right.$ $\left.312.9 \mathrm{~Hz},{ }^{2} J_{\mathrm{F}-\mathrm{C}}=40.2 \mathrm{~Hz}\right) ;{ }^{19} \mathrm{~F}$ NMR: $\delta=-62.75\left(\mathrm{t}, 2 \mathrm{~F},{ }^{3} J_{\mathrm{F}-\mathrm{F}}=8.0 \mathrm{~Hz}\right),-85.59\left(\mathrm{t}, 2 \mathrm{~F},{ }^{3} J_{\mathrm{F}-\mathrm{F}}=\right.$ 8.0 Hz). Anal. Calcd for $\mathrm{C}_{8} \mathrm{H}_{4} \mathrm{Br}_{2} \mathrm{~F}_{4} \mathrm{~S}$ : C, 26.11; H, 1.10. Found C, 26.38; H, 1.38.

2-(2-Bromo-1,1,2,2-tetrafluoro-ethylsulfanyl)-pyridine (3a): yellow liquid (85\% yield); $R_{f}=0.2$ (Pentane/ $\left.\mathrm{CH}_{2} \mathrm{Cl}_{2} 1: 1\right) ;{ }^{1} \mathrm{H}$ NMR: $\delta=8.66(\mathrm{~m}, 1 \mathrm{H}), 7.77-7.66(\mathrm{~m}, 2 \mathrm{H}), 7.36(\mathrm{ddd}, 1 \mathrm{H}$, $\left.{ }^{3} J_{\mathrm{H}-\mathrm{H}}=7.3 \mathrm{~Hz},{ }^{3} J_{\mathrm{H}-\mathrm{H}}=4.8 \mathrm{~Hz},{ }^{4} J_{\mathrm{H}-\mathrm{H}}=1.3 \mathrm{~Hz}\right) ;{ }^{13} \mathrm{C}$ NMR: $\delta=150.8,148.0,137.6,130.9$,

\footnotetext{
${ }^{1}$ a) Prakash, G. K. S.; Hu, J.; Olah, G. A. J. Org. Chem. 2003, 68, 4457-4463. b) Reutrakul, V.; Thongpaisanwong, T.; Tuchinda, P.; Kuhakarn, C.; Pohmakotr, M. J. Org. Chem. 2004, 69, 6913-6915.
} 
124.5, $123.1\left(\mathrm{tt},{ }^{1} J_{\mathrm{F}-\mathrm{C}}=292.3 \mathrm{~Hz},{ }^{2} J_{\mathrm{F}-\mathrm{C}}=34.3 \mathrm{~Hz}\right), 116.8\left(\mathrm{tt},{ }^{1} J_{\mathrm{F}-\mathrm{C}}=313.0 \mathrm{~Hz},{ }^{2} J_{\mathrm{F}-\mathrm{C}}=39.7 \mathrm{~Hz}\right)$; ${ }^{19} \mathrm{~F}$ NMR: $\delta=-63.12\left(\mathrm{t}, 2 \mathrm{~F},{ }^{3} J_{\mathrm{F}-\mathrm{F}}=8.0 \mathrm{~Hz}\right),-84.55\left(\mathrm{t}, 2 \mathrm{~F},{ }^{3} J_{\mathrm{F}-\mathrm{F}}=8.0 \mathrm{~Hz}\right)$. Anal. Calcd for $\mathrm{C}_{7} \mathrm{H}_{4} \mathrm{BrF}_{4} \mathrm{NS}$ : C, 28.98; H, 1.39. Found C, 29.12; H, 1.56.

[Difluoro-(4-fluoro-phenylsulfanyl)-methyl]-trimethyl-silane (4b): yellow liquid (95\% yield); $R_{f}=0.6$ (Pentane); ${ }^{1} \mathrm{H}$ NMR: $\delta=7.56(\mathrm{~m}, 2 \mathrm{H}), 7.06(\mathrm{~m}, 2 \mathrm{H}), 0.26(\mathrm{~s}, 9 \mathrm{H}) ;{ }^{13} \mathrm{C} \mathrm{NMR}$ : $\delta=169.9\left(\mathrm{~d},{ }^{1} J_{\mathrm{F}-\mathrm{C}}=249.7 \mathrm{~Hz}\right), 138.8\left(\mathrm{~d},{ }^{3} J_{\mathrm{F}-\mathrm{C}}=8.8 \mathrm{~Hz}\right), 133.9\left(\mathrm{td},{ }^{1} J_{\mathrm{F}-\mathrm{C}}=300.5 \mathrm{~Hz},{ }^{6} J_{\mathrm{F}-\mathrm{C}}=\right.$ $1.1 \mathrm{~Hz}), 121.5\left(\mathrm{td},{ }^{3} J_{\mathrm{F}-\mathrm{C}}=4.4 \mathrm{~Hz},{ }^{4} J_{\mathrm{F}-\mathrm{C}}=3.3 \mathrm{~Hz}\right), 116.1\left(\mathrm{~d},{ }^{2} J_{\mathrm{F}-\mathrm{C}}=21.9 \mathrm{~Hz}\right),-4.1\left(\mathrm{t},{ }^{3} J_{\mathrm{F}-\mathrm{C}}=\right.$ $1.4 \mathrm{~Hz}) ;{ }^{19} \mathrm{~F}$ NMR: $\delta=-88.41(\mathrm{~s}, 2 \mathrm{~F}),-112.06(\mathrm{~m}, 1 \mathrm{~F})$. Anal. Calcd for $\mathrm{C}_{10} \mathrm{H}_{13} \mathrm{~F}_{3} \mathrm{SSi}: \mathrm{C}, 47.97$; H, 5.23. Found C, 48.12; H, 5.49.

Trimethyl-(1,1,2,2-tetrafluoro-2-phenylsulfanyl-ethyl)-silane (5a): yellow liquid (90\% yield); $R_{f}=0.7$ (Pentane); ${ }^{1} \mathrm{H}$ NMR: $\delta=7.65(\mathrm{~m}, 2 \mathrm{H}), 7.48-7.36(\mathrm{~m}, 3 \mathrm{H}), 0.27(\mathrm{~m}, 9 \mathrm{H}) ;{ }^{13} \mathrm{C}$ NMR: $\delta=137.3,130.3,129.2,127.5\left(\mathrm{tt},{ }^{1} J_{\mathrm{F}-\mathrm{C}}=281.8 \mathrm{~Hz},{ }^{2} J_{\mathrm{F}-\mathrm{C}}=32.5 \mathrm{~Hz}\right), 124.6(\mathrm{~m}), 123.0$ $\left(\mathrm{tt},{ }^{1} J_{\mathrm{F}-\mathrm{C}}=273.2 \mathrm{~Hz},{ }^{2} J_{\mathrm{F}-\mathrm{C}}=45.3 \mathrm{~Hz}\right),-4.0(\mathrm{~m}) ;{ }^{19} \mathrm{~F}$ NMR: $\delta=-82.88\left(\mathrm{t}, 2 \mathrm{~F},{ }^{3} J_{\mathrm{F}-\mathrm{F}}=4.6 \mathrm{~Hz}\right),-$ $122.56\left(\mathrm{t}, 2 \mathrm{~F},{ }^{3} \mathrm{~J}_{\mathrm{F}-\mathrm{F}}=4.6 \mathrm{~Hz}\right)$. Anal. Calcd for $\mathrm{C}_{11} \mathrm{H}_{14} \mathrm{~F}_{4} \mathrm{SSi}$ : C, 46.79; H, 5.00. Found C, 47.01; H, 5.28.

Trimethyl-[1,1,2,2-tetrafluoro-2-(4-fluoro-phenyl-sulfanyl)-ethyl]-silane $\quad(5 \mathrm{~b})$ : yellow liquid (88\% yield); $R_{f}=0.6$ (Pentane); ${ }^{1} \mathrm{H}$ NMR: $\delta=7.64(\mathrm{~m}, 2 \mathrm{H}), 7.09(\mathrm{~m}, 2 \mathrm{H}), 0.27(\mathrm{~s}, 9 \mathrm{H})$; ${ }^{13} \mathrm{C}$ NMR: $\delta=164.4\left(\mathrm{~d},{ }^{1} J_{\mathrm{F}-\mathrm{C}}=251.4 \mathrm{~Hz}\right), 139.5\left(\mathrm{~d},{ }^{3} J_{\mathrm{F}-\mathrm{C}}=8.8 \mathrm{~Hz}\right), 127.1\left(\mathrm{ttd},{ }^{1} J_{\mathrm{F}-\mathrm{C}}=281.2\right.$ $\left.\mathrm{Hz},{ }^{2} J_{\mathrm{F}-\mathrm{C}}=32.9 \mathrm{~Hz},{ }^{6} J_{\mathrm{F}-\mathrm{C}}=1.4 \mathrm{~Hz}\right), 122.8\left(\mathrm{tt},{ }^{1} J_{\mathrm{F}-\mathrm{C}}=272.2 \mathrm{~Hz},{ }^{2} J_{\mathrm{F}-\mathrm{C}}=45.3 \mathrm{~Hz}\right), 119.9(\mathrm{~m})$, $116.5\left(\mathrm{~d},{ }^{2} J_{\mathrm{F}-\mathrm{C}}=22.0 \mathrm{~Hz}\right)-4.0(\mathrm{~m}) ;{ }^{19} \mathrm{~F}$ NMR: $\delta=-83.29\left(\mathrm{t}, 2 \mathrm{~F},{ }^{3} J_{\mathrm{F}-\mathrm{F}}=4.6 \mathrm{~Hz}\right),-110.55(\mathrm{~m}$, $1 \mathrm{~F}),-122.52\left(\mathrm{t}, 2 \mathrm{~F},{ }^{3} J_{\mathrm{F}-\mathrm{F}}=4.6 \mathrm{~Hz}\right)$. Anal. Calcd for $\mathrm{C}_{11} \mathrm{H}_{13} \mathrm{~F}_{5} \mathrm{SSi}$ : C, 43.99; H, 4.36. Found C, $44.22 ; \mathrm{H}, 4.48$.

2-(1,1,2,2-Tetrafluoro-2-trimethylsilanyl-ethylsulfanyl)-pyridine (6a): yellow liquid (76\% yield); $R_{f}=0.5$ (Pentane/ $\left.\mathrm{CH}_{2} \mathrm{Cl}_{2} 1: 2\right) ;{ }^{1} \mathrm{H} \mathrm{NMR}: \delta=8.68\left(\mathrm{ddd}, 1 \mathrm{H},{ }^{3} J_{\mathrm{H}-\mathrm{H}}=4.8 \mathrm{~Hz},{ }^{4} J_{\mathrm{H}-\mathrm{H}}=1.6\right.$ $\left.\mathrm{Hz},{ }^{5} J_{\mathrm{H}-\mathrm{H}}=1.1 \mathrm{~Hz}\right), 7.71-7.63(\mathrm{~m}, 2 \mathrm{H}), 7.26\left(\mathrm{ddd}, 1 \mathrm{H},{ }^{3} J_{\mathrm{H}-\mathrm{H}}=6.5 \mathrm{~Hz},{ }^{3} J_{\mathrm{H}-\mathrm{H}}=4.8 \mathrm{~Hz},{ }^{4} J_{\mathrm{H}-\mathrm{H}}=\right.$ $2.2 \mathrm{~Hz}), 0.28(\mathrm{~s}, 9 \mathrm{H}) ;{ }^{13} \mathrm{C} \mathrm{NMR}: \delta=150.5,149.8,137.3,130.2,127.8\left(\mathrm{tt},{ }^{1} J_{\mathrm{F}-\mathrm{C}}=283.4 \mathrm{~Hz}\right.$, $\left.{ }^{2} J_{\mathrm{F}-\mathrm{C}}=32.4 \mathrm{~Hz}\right), 123.6,122.5\left(\mathrm{tt},{ }^{1} J_{\mathrm{F}-\mathrm{C}}=273.0 \mathrm{~Hz},{ }^{2} J_{\mathrm{F}-\mathrm{C}}=44.7 \mathrm{~Hz}\right),-4.1(\mathrm{~m}) ;{ }^{19} \mathrm{~F}$ NMR: $\delta=-$ $82.15\left(\mathrm{t}, 2 \mathrm{~F},{ }^{3} J_{\mathrm{F}-\mathrm{F}}=4.6 \mathrm{~Hz}\right),-122.48\left(\mathrm{t}, 2 \mathrm{~F},{ }^{3} \mathrm{~J}_{\mathrm{F}-\mathrm{F}}=4.6 \mathrm{~Hz}\right)$. Anal. Calcd for $\mathrm{C}_{10} \mathrm{H}_{13} \mathrm{~F}_{4} \mathrm{NSSi}: \mathrm{C}$, 42.39; H, 4.62. Found C, 42.54; H, 4.76. 
Synthesis of (2,2-Difluoro-1-phenyl-vinyloxy)-trimethyl-silane (9): ${ }^{2}$ Into a mixture of $\mathrm{Mg}$ turnings $(0.2 \mathrm{~g}, 8.3 \mathrm{mmol})$ and $\mathrm{TMSCl}(2 \mathrm{~mL}, 16 \mathrm{mmol})$ in anhydrous $\mathrm{THF}(10 \mathrm{~mL})$ at $0^{\circ} \mathrm{C}$, was slowly added $\mathrm{PhCOCF}_{3}(0.56 \mathrm{~mL}, 4 \mathrm{mmol})$. The reaction mixture was stirred at $0^{\circ} \mathrm{C}$ for 4 h. After removal of the solvent and excess TMSCl under vacuum, pentane was added. Solids were filtrated and the resulting filtrate was concentrated to give $0.92 \mathrm{~g}$ of crude $\mathbf{9}$ as a colorless liquid. The product is pure enough to be used for the next step. $R_{f}=0.7$ (Petroleum ether/ $\left.\mathrm{CH}_{2} \mathrm{Cl}_{2} 7: 3\right) ;{ }^{1} \mathrm{H}$ NMR: $\delta=7.62(\mathrm{~m}, 2 \mathrm{H}), 7.48(\mathrm{~m}, 2 \mathrm{H}), 7.38(\mathrm{~m}, 1 \mathrm{H}), 0.22(\mathrm{~s}, 9 \mathrm{H}) ;{ }^{19} \mathrm{~F}$ NMR: $\delta=-100.29\left(\mathrm{~d}, 1 \mathrm{~F},{ }^{2} J_{\mathrm{F}-\mathrm{F}}=67.7 \mathrm{~Hz}\right),-112.06\left(\mathrm{~d}, 1 \mathrm{~F},{ }^{2} J_{\mathrm{F}-\mathrm{F}}=67.7 \mathrm{~Hz}\right)$.

1,1,2,2-Tetrafluoro-2-(4-fluoro-phenylsulfanyl)-ethane-sulfonyl fluoride (13b): yellow liquid (A: 67\% yield, B: 64\% yield); $R_{f}=0.7$ (Pentane); ${ }^{1} \mathrm{H}$ NMR: $\delta=7.66(\mathrm{~m}, 2 \mathrm{H}), 7.14(\mathrm{~m}$, $2 \mathrm{H}) ;{ }^{13} \mathrm{C}$ NMR: $\delta=165.4\left(\mathrm{~d},{ }^{1} J_{\mathrm{F}-\mathrm{C}}=253.6 \mathrm{~Hz}\right), 140.0\left(\mathrm{~d},{ }^{3} J_{\mathrm{F}-\mathrm{C}}=9.3 \mathrm{~Hz}\right), 117.6(\mathrm{~m}), 117.4(\mathrm{~d}$, $\left.{ }^{2} J_{\mathrm{F}-\mathrm{C}}=22.0 \mathrm{~Hz}\right), 121.8(\mathrm{~m}), 116.1\left(\mathrm{ttd},{ }^{1} J_{\mathrm{F}-\mathrm{C}}=300.6 \mathrm{~Hz},{ }^{2} J_{\mathrm{F}-\mathrm{C}}=40.3 \mathrm{~Hz},{ }^{2} J_{\mathrm{F}-\mathrm{C}}=33.8 \mathrm{~Hz}\right) ;{ }^{19} \mathrm{~F}$ NMR: $\delta=46.01(\mathrm{~m}, 1 \mathrm{~F}),-86.99(\mathrm{~m}, 2 \mathrm{~F}),-105.70(\mathrm{~m}, 2 \mathrm{~F}),-107.67(\mathrm{~m}, 1 \mathrm{~F}) ; \mathrm{MS}(\mathrm{EI}): m / z=$ 127.3, 177.3, $310.2\left(\mathrm{M}^{+}\right)$; HRMS: calcd for $\mathrm{C}_{8} \mathrm{H}_{4} \mathrm{~F}_{6} \mathrm{O}_{2} \mathrm{~S}_{2}$ : 309.9557 ; found: 309.9559 .

\section{1,1,2,2-Tetrafluoro-2-(4-trimethylsilanyl-phenylsulfanyl)-ethanesulfonyl fluoride (13c):} colorless liquid (A: $48 \%$ yield, B: $77 \%$ yield); $R_{f}=0.7$ (Pentane); ${ }^{1} \mathrm{H}$ NMR: $\delta=7.64-7.56(\mathrm{~m}$, 4H), 0.29 (s, 9H); ${ }^{13} \mathrm{C}$ NMR: $\delta=145.4,136.5,134.6,122.4\left(\mathrm{t},{ }^{3} J_{\mathrm{F}-\mathrm{C}}=3.0 \mathrm{~Hz}\right), 121.9\left(\mathrm{ttd},{ }^{1} J_{\mathrm{F}-}\right.$ $\left.\mathrm{C}=291.0 \mathrm{~Hz},{ }^{2} J_{\mathrm{F}-\mathrm{C}}=30.8 \mathrm{~Hz},{ }^{3} J_{\mathrm{F}-\mathrm{C}}=1.1 \mathrm{~Hz}\right), 116.0\left(\mathrm{ttd},{ }^{1} J_{\mathrm{F}-\mathrm{C}}=300.5 \mathrm{~Hz},{ }^{2} J_{\mathrm{F}-\mathrm{C}}=40.8 \mathrm{~Hz},{ }^{2} J_{\mathrm{F}-\mathrm{C}}\right.$ $=32.6 \mathrm{~Hz}),-1.2 ;{ }^{19} \mathrm{~F}$ NMR: $\delta=46.01(\mathrm{~m}, 1 \mathrm{~F}),-86.40$ (m, 2F), -105.54 (m, 2F); MS (EI): $m / z$ $=265,349,364\left(\mathrm{M}^{+}\right)$; HRMS: calcd for $\mathrm{C}_{11} \mathrm{H}_{13} \mathrm{~F}_{5} \mathrm{O}_{2} \mathrm{~S}_{2} \mathrm{Si}$ : 364.0046; found: 364.0045 .

2-(4-Bromo-phenylsulfanyl)-1,1,2,2-tetrafluoro-ethane-sulfonyl fluoride (13d): colorless liquid (B: $62 \%$ yield); $R_{f}=0.7$ (Pentane); ${ }^{1} \mathrm{H}$ NMR: $\delta=7.64-7.51(\mathrm{~m}, 4 \mathrm{H}) ;{ }^{13} \mathrm{C}$ NMR: $\delta=$ 138.8, 133.2, $127.1,121.5\left(\mathrm{ttd},{ }^{1} J_{\mathrm{F}-\mathrm{C}}=291.6 \mathrm{~Hz},{ }^{2} J_{\mathrm{F}-\mathrm{C}}=30.8 \mathrm{~Hz},{ }^{3} J_{\mathrm{F}-\mathrm{C}}=1.0 \mathrm{~Hz}\right), 121.1(\mathrm{~m})$, $115.8\left(\mathrm{ttd},{ }^{1} J_{\mathrm{F}-\mathrm{C}}=300.6 \mathrm{~Hz},{ }^{2} J_{\mathrm{F}-\mathrm{C}}=40.4 \mathrm{~Hz},{ }^{2} J_{\mathrm{F}-\mathrm{C}}=32.9 \mathrm{~Hz}\right) ;{ }^{19} \mathrm{~F}$ NMR: $\delta=46.06(\mathrm{~m}, 1 \mathrm{~F}),-$ $86.59(\mathrm{~m}, 2 \mathrm{~F}),-105.72(\mathrm{~m}, 2 \mathrm{~F})$; MS (EI): $\mathrm{m} / z=187,189,237,239,370\left(\mathrm{M}^{+}\right), 372$; HRMS: calcd for $\mathrm{C}_{8} \mathrm{H}_{4} \mathrm{BrF}_{5} \mathrm{O}_{2} \mathrm{~S}_{2}: 369.8756$; found: 369.8757 .

1,1,2,2-Tetrafluoro-2-(pyridin-2-ylsulfanyl)-ethane-sulfonyl fluoride (14a): yellow liquid (A: $70 \%$ yield, B: $72 \%$ yield); $R_{f}=0.3$ (Pentane); ${ }^{1} \mathrm{H}$ NMR: $\delta=8.68\left(\mathrm{ddd}, 1 \mathrm{H},{ }^{3} J_{\mathrm{H}-\mathrm{H}}=4.8 \mathrm{~Hz}\right.$, $\left.{ }^{4} J_{\mathrm{H}-\mathrm{H}}=1.9 \mathrm{~Hz},{ }^{5} J_{\mathrm{H}-\mathrm{H}}=0.9 \mathrm{~Hz}\right), 7.78\left(\mathrm{ddd}, 1 \mathrm{H},{ }^{3} J_{\mathrm{H}-\mathrm{H}}={ }^{3} J_{\mathrm{H}-\mathrm{H}}=7.5 \mathrm{~Hz},{ }^{4} J_{\mathrm{H}-\mathrm{H}}=1.9 \mathrm{~Hz}\right), 7.72(\mathrm{~m}$,

2 a) Amii, H.; Kobayashi, T.; Hatamoto, Y.; Uneyama, K. Chem. Commun. 1999, 1323-1324. b) Prakash, G. K. S.; Hu, J.; Simon, J.; Bellew, D. R.; Olah, G. A. J. Fluorine. Chem. 2004, 125, 595-601. 
$1 \mathrm{H}), 7.41\left(\mathrm{ddd}, 1 \mathrm{H},{ }^{3} J_{\mathrm{H}-\mathrm{H}}=7.5 \mathrm{~Hz},{ }^{3} J_{\mathrm{H}-\mathrm{H}}=4.8 \mathrm{~Hz},{ }^{4} J_{\mathrm{H}-\mathrm{H}}=1.2 \mathrm{~Hz}\right) ;{ }^{13} \mathrm{C}$ NMR: $\delta=151.2$, 146.5 (m), 138.0, 131.5, 125.1, 126.7-114.7 (m, 2C); ${ }^{19} \mathrm{~F} \mathrm{NMR:} \delta=46.34$ (m, 1F), -85.23 (m, 2F), -105.74 (m, 2F); MS (EI): $m / z=293.4\left(\mathrm{M}^{+}\right)$; HRMS: calcd for $\mathrm{C}_{7} \mathrm{H}_{4} \mathrm{~F}_{5} \mathrm{NO}_{2} \mathrm{~S}_{2}: 292.9604$; found: 292.9608 .

1,1-Difluoro-2-oxo-2-phenyl-ethanesulfonyl fluoride (15): colorless liquid (A: 45\% yield, B: $67 \%$ yield); $R_{f}=0.6$ (Petroleum ether/ $\mathrm{CH}_{2} \mathrm{Cl}_{2} 9: 1$ ); ${ }^{1} \mathrm{H}$ NMR: $\delta=8.10(\mathrm{~m}, 2 \mathrm{H}), 7.79$ (m, $1 \mathrm{H}), 7.60(\mathrm{~m}, 2 \mathrm{H}) ;{ }^{13} \mathrm{C} \mathrm{NMR}: \delta=181.1\left(\mathrm{td},{ }^{2} J_{\mathrm{F}-\mathrm{C}}=23.9 \mathrm{~Hz},{ }^{3} J_{\mathrm{F}-\mathrm{C}}=1.7 \mathrm{~Hz}\right), 136.8,130.5(\mathrm{t}$, $\left.{ }^{4} J_{\mathrm{F}-\mathrm{C}}=2.7 \mathrm{~Hz}\right), 129.8\left(\mathrm{td},{ }^{3} J_{\mathrm{F}-\mathrm{C}}={ }^{4} J_{\mathrm{F}-\mathrm{C}}=2.6 \mathrm{~Hz}\right), 116.6\left(\mathrm{td},{ }^{1} J_{\mathrm{F}-\mathrm{C}}=309.3 \mathrm{~Hz},{ }^{2} J_{\mathrm{F}-\mathrm{C}}=28.0 \mathrm{~Hz}\right)$; ${ }^{19} \mathrm{~F}$ NMR: $\delta=41.61\left(\mathrm{t}, 1 \mathrm{~F},{ }^{3} J_{\mathrm{F}-\mathrm{F}}=2.3 \mathrm{~Hz}\right),-95.98\left(\mathrm{~d}, 2 \mathrm{~F},{ }^{3} J_{\mathrm{F}-\mathrm{F}}=2.3 \mathrm{~Hz}\right)$; MS (EI): $m / z=77.3$, 105.3, $238.3\left(\mathrm{M}^{+}\right)$; HRMS: calcd for $\mathrm{C}_{8} \mathrm{H}_{5} \mathrm{~F}_{3} \mathrm{O}_{3} \mathrm{~S}$ : 237.9912; found: 237.9913 .

Difluoro-phenylsulfanyl-methanesulfonyl fluoride (16a): colorless liquid (B: 57\% yield); $R_{f}=0.6$ (Pentane); ${ }^{1} \mathrm{H}$ NMR: $\delta=7.71\left(\mathrm{~d}, 2 \mathrm{H},{ }^{3} J_{\mathrm{H}-\mathrm{H}}=7.5 \mathrm{~Hz}\right), 7.56\left(\mathrm{t}, 1 \mathrm{H},{ }^{3} J_{\mathrm{H}-\mathrm{H}}=7.5 \mathrm{~Hz}\right), 7.46$ $\left(\mathrm{dd}, 2 \mathrm{H},{ }^{3} J_{\mathrm{H}-\mathrm{H}}={ }^{3} J_{\mathrm{H}-\mathrm{H}}=7.5 \mathrm{~Hz}\right) ;{ }^{13} \mathrm{C}$ NMR: $\delta=137.5\left(\mathrm{t},{ }^{4} J_{\mathrm{F}-\mathrm{C}}=1.1 \mathrm{~Hz}\right), 132.1,130.0,126.6$ $\left(\mathrm{td},{ }^{1} J_{\mathrm{F}-\mathrm{C}}=323.3 \mathrm{~Hz},{ }^{2} J_{\mathrm{F}-\mathrm{C}}=32.9 \mathrm{~Hz}\right), 121.8\left(\mathrm{t},{ }^{3} J_{\mathrm{F}-\mathrm{C}}=3.0 \mathrm{~Hz}\right) ;{ }^{19} \mathrm{~F}$ NMR: $\delta=35.06\left(\mathrm{t}, 1 \mathrm{~F},{ }^{3} J_{\mathrm{F}-}\right.$ $\mathrm{F}=4.6 \mathrm{~Hz}),-76.08\left(\mathrm{~d}, 2 \mathrm{~F},{ }^{3} J_{\mathrm{F}-\mathrm{F}}=4.6 \mathrm{~Hz}\right) ; \mathrm{MS}(\mathrm{EI}): \mathrm{m} / z=77,109,159,242\left(\mathrm{M}^{+}\right)$; HRMS: calcd for $\mathrm{C}_{7} \mathrm{H}_{5} \mathrm{~F}_{3} \mathrm{O}_{2} \mathrm{~S}_{2}$ : 241.9683; found: 241.9686 .

Difluoro-(4-fluoro-phenylsulfanyl)-methanesulfonyl fluo-ride (16b): colorless liquid (B: $32 \%$ yield); $\quad R_{f}=0.7$ (Pentane); ${ }^{1} \mathrm{H}$ NMR: $\delta=7.70(\mathrm{~m}, 2 \mathrm{H}), 7.16(\mathrm{~m}, 2 \mathrm{H}) ;{ }^{13} \mathrm{C}$ NMR: $\delta=$ $165.3\left(\mathrm{~d},{ }^{1} J_{\mathrm{F}-\mathrm{C}}=254.7 \mathrm{~Hz}\right), 139.9\left(\mathrm{~d},{ }^{3} J_{\mathrm{F}-\mathrm{C}}=9.3 \mathrm{~Hz}\right), 126.7\left(\mathrm{tdd},{ }^{1} J_{\mathrm{F}-\mathrm{C}}=323.4 \mathrm{~Hz},{ }^{2} J_{\mathrm{F}-\mathrm{C}}=32.3\right.$ $\left.\mathrm{Hz},{ }^{6} J_{\mathrm{F}-\mathrm{C}}=2.4 \mathrm{~Hz}\right), 117.4\left(\mathrm{~d},{ }^{2} J_{\mathrm{F}-\mathrm{C}}=22.5 \mathrm{~Hz}\right), 117.1(\mathrm{~m}) ;{ }^{19} \mathrm{~F} \mathrm{NMR}: \delta=35.2\left(\mathrm{t}, 1 \mathrm{~F},{ }^{3} J_{\mathrm{F}-\mathrm{F}}=\right.$ $4.6 \mathrm{~Hz}),-76.80\left(\mathrm{~d}, 2 \mathrm{~F},{ }^{3} J_{\mathrm{F}-\mathrm{F}}=4.6 \mathrm{~Hz}\right),-106.74(\mathrm{~m}, 1 \mathrm{~F}) ; \mathrm{MS}(\mathrm{EI}): \mathrm{m} / z=177,260\left(\mathrm{M}^{+}\right)$; HRMS: calcd for $\mathrm{C}_{7} \mathrm{H}_{4} \mathrm{~F}_{4} \mathrm{O}_{2} \mathrm{~S}_{2}$ : 259.9589; found: 259.9591 .

Benzenesulfinyl-difluoro-methanesulfonyl fluoride (19a): colorless liquid (34\% yield); $R_{f}=0.45$ (Pentane/ $\mathrm{CH}_{2} \mathrm{Cl}_{2}$ 1:1); ${ }^{1} \mathrm{H}$ NMR: $\delta=7.86\left(\mathrm{~d}, 2 \mathrm{H},{ }^{3} J_{\mathrm{H}-\mathrm{H}}=7.3 \mathrm{~Hz}\right), 7.77-7.63(\mathrm{~m}, 3 \mathrm{H})$; ${ }^{13} \mathrm{C}$ NMR: $\delta=134.7,134.1\left(\mathrm{dd},{ }^{3} J_{\mathrm{F}-\mathrm{C}}={ }^{3} J_{\mathrm{F}-\mathrm{C}}=2.5 \mathrm{~Hz}\right), 129.9,126.6\left(\mathrm{dd},{ }^{4} J_{\mathrm{F}-\mathrm{C}}={ }^{4} J_{\mathrm{F}-\mathrm{C}}=1.1\right.$ $\mathrm{Hz}), 123.7\left(\mathrm{ddd},{ }^{1} J_{\mathrm{F}-\mathrm{C}}=348.9 \mathrm{~Hz},{ }^{1} J_{\mathrm{F}-\mathrm{C}}=340.7 \mathrm{~Hz},{ }^{2} J_{\mathrm{F}-\mathrm{C}}=24.8 \mathrm{~Hz}\right) ;{ }^{19} \mathrm{~F}$ NMR: $\delta=49.78(\mathrm{dd}$, $\left.1 \mathrm{~F},{ }^{3} J_{\mathrm{F}-\mathrm{F}}={ }^{3} J_{\mathrm{F}-\mathrm{F}}=5.1 \mathrm{~Hz}\right),-99.61\left(\mathrm{dd}, 1 \mathrm{~F},{ }^{1} J_{\mathrm{F}-\mathrm{F}}=215.7 \mathrm{~Hz},{ }^{3} J_{\mathrm{F}-\mathrm{F}}=5.1 \mathrm{~Hz}\right),-105.45(\mathrm{dd}, 1 \mathrm{~F}$, $\left.{ }^{1} J_{\mathrm{F}-\mathrm{F}}=215.7 \mathrm{~Hz},{ }^{3} J_{\mathrm{F}-\mathrm{F}}=5.1 \mathrm{~Hz}\right) ; \mathrm{MS}(\mathrm{EI}): \mathrm{m} / z=125,258\left(\mathrm{M}^{+}\right)$; HRMS: calcd for $\mathrm{C}_{7} \mathrm{H}_{5} \mathrm{~F}_{3} \mathrm{O}_{3} \mathrm{~S}_{2}$ : 257.9632; found: 257.9630 .

Benzenesulfonyl-difluoro-methanesulfonyl fluoride (20a): colorless liquid (75\% yield); $R_{f}=0.7$ (Pentane); ${ }^{1} \mathrm{H}$ NMR: $\delta=8.09\left(\mathrm{~d}, 2 \mathrm{H},{ }^{3} J_{\mathrm{H}-\mathrm{H}}=7.6 \mathrm{~Hz}\right), 7.90\left(\mathrm{t}, 1 \mathrm{H},{ }^{3} J_{\mathrm{H}-\mathrm{H}}=7.6 \mathrm{~Hz}\right), 7.70$ $\left(\mathrm{dd}, 2 \mathrm{H},{ }^{3} J_{\mathrm{H}-\mathrm{H}}={ }^{3} J_{\mathrm{H}-\mathrm{H}}=7.6 \mathrm{~Hz}\right) ;{ }^{13} \mathrm{C}$ NMR: $\delta=137.7,131.6,131.0,130.2,118.8\left(\mathrm{td},{ }^{1} J_{\mathrm{F}-\mathrm{C}}=\right.$ $\left.336.3 \mathrm{~Hz},{ }^{2} J_{\mathrm{F}-\mathrm{C}}=30.0 \mathrm{~Hz}\right) ;{ }^{19} \mathrm{~F}$ NMR: $\delta=49.28\left(\mathrm{t}, 1 \mathrm{~F},{ }^{3} J_{\mathrm{F}-\mathrm{F}}=5.7 \mathrm{~Hz}\right),-99.40\left(\mathrm{~d}, 2 \mathrm{~F},{ }^{3} J_{\mathrm{F}-\mathrm{F}}=\right.$ 
5.7 Hz); MS (EI): $m / z=77,141,274\left(\mathrm{M}^{+}\right)$; HRMS: calcd for $\mathrm{C}_{7} \mathrm{H}_{5} \mathrm{~F}_{3} \mathrm{O}_{4} \mathrm{~S}_{2}$ : 273.9581; found: 273.9580 .

Lithium 2-benzenesulfinyl-1,1,2,2-tetrafluoro-ethanesulfonate (22): white solid (0.15 g, $71 \%$ yield); m.p. $200^{\circ} \mathrm{C},{ }^{1} \mathrm{H}$ NMR (acetone-d $\left.{ }_{6}\right): \delta=7.87\left(\mathrm{~d}, 2 \mathrm{H},{ }^{3} J_{\mathrm{H}-\mathrm{H}}=7.2 \mathrm{~Hz}\right), 7.70-7.61(\mathrm{~m}$, $3 \mathrm{H}), 4.2$ (br s, $\mathrm{H}_{2} \mathrm{O}$ linked); ${ }^{13} \mathrm{C}$ NMR (acetone-d $\left.{ }_{6}\right): \delta=137.6\left(\mathrm{dd},{ }^{3} J_{\mathrm{F}-\mathrm{C}}={ }^{3} J_{\mathrm{F}-\mathrm{C}}=2.5 \mathrm{~Hz}\right.$ ), 133.9, 130.0, $127.7\left(\mathrm{~d},{ }^{4} J_{\mathrm{F}-\mathrm{C}}=1.6 \mathrm{~Hz}\right), 119.2\left(\mathrm{dddd},{ }^{1} J_{\mathrm{F}-\mathrm{C}}=310.5 \mathrm{~Hz},{ }^{1} J_{\mathrm{F}-\mathrm{C}}=309.6 \mathrm{~Hz},{ }^{2} J_{\mathrm{F}-\mathrm{C}}=\right.$ $\left.{ }^{2} J_{\mathrm{F}-\mathrm{C}}=30.5 \mathrm{~Hz}\right), 115.2\left(\mathrm{dddd},{ }^{1} J_{\mathrm{F}-\mathrm{C}}={ }^{1} J_{\mathrm{F}-\mathrm{C}}=289.5 \mathrm{~Hz},{ }^{2} J_{\mathrm{F}-\mathrm{C}}={ }^{2} J_{\mathrm{F}-\mathrm{C}}=27.8 \mathrm{~Hz}\right) ;{ }^{19} \mathrm{~F} \mathrm{NMR}$ (acetone-d $\left.\mathrm{d}_{6}\right): \delta=-113.47\left(\mathrm{ddd}, 1 \mathrm{~F},{ }^{2} J_{\mathrm{F}-\mathrm{F}}=265.8 \mathrm{~Hz},{ }^{3} J_{\mathrm{F}-\mathrm{F}}={ }^{3} J_{\mathrm{F}-\mathrm{F}}=4.7 \mathrm{~Hz}\right),-113.51(\mathrm{ddd}, 1 \mathrm{~F}$, $\left.{ }^{2} J_{\mathrm{F}-\mathrm{F}}=233.7 \mathrm{~Hz},{ }^{3} J_{\mathrm{F}-\mathrm{F}}={ }^{3} J_{\mathrm{F}-\mathrm{F}}=4.7 \mathrm{~Hz}\right),-114.49\left(\mathrm{ddd}, 1 \mathrm{~F},{ }^{2} J_{\mathrm{F}-\mathrm{F}}=265.8 \mathrm{~Hz},{ }^{3} J_{\mathrm{F}-\mathrm{F}}={ }^{3} J_{\mathrm{F}-\mathrm{F}}=4.7\right.$ $\mathrm{Hz}),-120.80\left(\mathrm{ddd}, 1 \mathrm{~F},{ }^{2} J_{\mathrm{F}-\mathrm{F}}=233.7 \mathrm{~Hz},{ }^{3} J_{\mathrm{F}-\mathrm{F}}={ }^{3} J_{\mathrm{F}-\mathrm{F}}=4.7 \mathrm{~Hz}\right) ; \mathrm{MS}(\mathrm{ESI}-\mathrm{MeOH}): \mathrm{m} / z=304.9$ $\left(\mathrm{M}^{-}\right), 305.9,306.9,616.8$.

Lithium 2-benzenesulfonyl-1,1,2,2-tetrafluoro-ethanesulfonate (23): white solid (0.19 g, $92 \%$ yield); m.p. $75-80{ }^{\circ} \mathrm{C} ;{ }^{1} \mathrm{H}$ NMR (acetone- $\mathrm{d}_{6}$ ): $\delta=8.05$ (d, $2 \mathrm{H},{ }^{3} J_{\mathrm{H}-\mathrm{H}}=7.5 \mathrm{~Hz}$ ), $7.94(\mathrm{~m}$, 1H), 7.79 (m, 2H), 3.01 (br s, $\mathrm{H}_{2} \mathrm{O}$ linked); ${ }^{13} \mathrm{C}$ NMR (acetone-d $\mathrm{d}_{6}$ ): $\delta=137.1,134.2,131.4$, 130.6, $116.4\left(\mathrm{tt},{ }^{1} J_{\mathrm{F}-\mathrm{C}}=279.9 \mathrm{~Hz},{ }^{2} J_{\mathrm{F}-\mathrm{C}}=37.1 \mathrm{~Hz},{ }^{3} J_{\mathrm{F}-\mathrm{C}}=35.0 \mathrm{~Hz}\right), 115.4\left(\mathrm{tt},{ }^{1} J_{\mathrm{F}-\mathrm{C}}=287.8 \mathrm{~Hz}\right.$, ${ }^{2} J_{\mathrm{F}-\mathrm{C}}=33.9 \mathrm{~Hz}$ ); ${ }^{19} \mathrm{~F}$ NMR (acetone- $\left.\mathrm{d}_{6}\right): \delta=-110.65(\mathrm{~m}, 2 \mathrm{~F}),-113.48$ (m, 2F); MS (ESI$\mathrm{MeOH}): m / z=321\left(\mathbf{M}^{-}\right), 322,323$.

Lithium difluoro-phenylsulfanyl-methanesulfonate (24): White solid (1.29 g, $83 \%$ yield); m.p. $175-182^{\circ} \mathrm{C} ;{ }^{1} \mathrm{H}$ NMR (acetone-d $\mathrm{d}_{6}$ ) : $\delta=7.63$ (m, 2H), 7.49-7.39 (m, 3H), 2.88 (br s, $\mathrm{H}_{2} \mathrm{O}$ linked); ${ }^{13} \mathrm{C}$ NMR (acetone- $\left.\mathrm{d}_{6}\right): \delta=137.0,130.5,129.8,128.2\left(\mathrm{t},{ }^{1} J_{\mathrm{F}-\mathrm{C}}=315.3 \mathrm{~Hz}\right), 127.3(\mathrm{t}$, $\left.{ }^{3} J_{\mathrm{F}-\mathrm{C}}=1.9 \mathrm{~Hz}\right) ;{ }^{19} \mathrm{~F}$ NMR $\left(\right.$ acetone-d $\left._{6}\right): \delta=-79.08(\mathrm{~s}, 2 \mathrm{~F}) ; \mathrm{MS}(\mathrm{ESI}-\mathrm{MeOH}): \mathrm{m} / \mathrm{z}=239.0\left(\mathrm{M}^{-}\right)$, 240.0, 241.0, 484.9.

Lithium benzenesulfinyl-difluoro-methanesulfonate (25): White solid ( $0.16 \mathrm{~g}, 51 \%$ yield); m.p. $215-220^{\circ} \mathrm{C} ;{ }^{1} \mathrm{H}$ NMR (acetone- $\mathrm{d}_{6}$ ): $\delta=7.83\left(\mathrm{~d}, 2 \mathrm{H}^{3} J_{\mathrm{H}-\mathrm{H}}=7.7 \mathrm{~Hz}\right), 7.72-7.62(\mathrm{~m}, 3 \mathrm{H})$; ${ }^{13} \mathrm{C}$ NMR (acetone-d $\left.\mathrm{d}_{6}\right): \delta=136.3\left(\mathrm{dd},{ }^{3} J_{\mathrm{F}-\mathrm{C}}={ }^{3} J_{\mathrm{F}-\mathrm{C}}=3.0 \mathrm{~Hz}\right), 133.9,130.0,127.5(\mathrm{~m}), 122.6$ $\left(\mathrm{dd},{ }^{1} J_{\mathrm{F}-\mathrm{C}}=341.9 \mathrm{~Hz},{ }^{1} J_{\mathrm{F}-\mathrm{C}}=329.9 \mathrm{~Hz}\right) ;{ }^{19} \mathrm{~F}$ NMR $\left(\right.$ acetone-d $\left.\mathrm{d}_{6}\right): \delta=-105.90\left(\mathrm{~d}, 1 \mathrm{~F},{ }^{1} J_{\mathrm{F}-\mathrm{F}}=\right.$ $205.9 \mathrm{~Hz}),-113.87\left(\mathrm{~d}, 1 \mathrm{~F},{ }^{1} J_{\mathrm{F}-\mathrm{F}}=205.9 \mathrm{~Hz}\right)$; MS $(\mathrm{ESI}-\mathrm{MeOH}): \mathrm{m} / z=130.0,131.1,255.0\left(\mathrm{M}^{-}\right.$ ), 256.0, 256.9.

Lithium benzenesulfonyl-difluoro-methanesulfonate (26): White solid ( $0.20 \mathrm{~g}, 72 \%$ yield); m.p. $225^{\circ} \mathrm{C} ;{ }^{1} \mathrm{H}$ NMR (acetone- $\left.\mathrm{d}_{6}\right): \delta=8.02(\mathrm{~m}, 2 \mathrm{H}), 7.83(\mathrm{~m}, 1 \mathrm{H}), 7.63(\mathrm{~m}, 2 \mathrm{H}), 3.20$ (br s, $\mathrm{H}_{2} \mathrm{O}$ linked); ${ }^{13} \mathrm{C}$ NMR (acetone- $\left.\mathrm{d}_{6}\right): \delta=136.1,135.2,131.5\left(\mathrm{t},{ }^{4} J_{\mathrm{F}-\mathrm{C}}=1.1 \mathrm{~Hz}\right), 129.9,119.9$ $\left(\mathrm{t},{ }^{1} J_{\mathrm{F}-\mathrm{C}}=324.7 \mathrm{~Hz}\right) ;{ }^{19} \mathrm{~F}$ NMR $\left(\right.$ acetone-d $\left._{6}\right): \delta=-104.76(\mathrm{~s}, 2 \mathrm{~F}) ; \mathrm{MS}(\mathrm{ESI}-\mathrm{MeOH}): \mathrm{m} / \mathrm{z}=$ 130.0, $271.0\left(\mathrm{M}^{-}\right), 272.0,273.0$. 
Lithium 1,1-difluoro-2-oxo-2-phenyl-ethanesulfonate (27): White solid (0.94 g, 77\% yield); m.p. $118-122^{\circ} \mathrm{C} ;{ }^{1} \mathrm{H}$ NMR (acetone- $\left.\mathrm{d}_{6}\right): \delta=8.25(\mathrm{~m}, 2 \mathrm{H}), 7.67(\mathrm{~m}, 1 \mathrm{H}), 7.51(\mathrm{~m}, 2 \mathrm{H})$, 4.06 (br s, $\mathrm{H}_{2} \mathrm{O}$ linked); ${ }^{13} \mathrm{C}$ NMR (acetone- $\left.\mathrm{d}_{6}\right): \delta=188.8\left(\mathrm{t},{ }^{2} J_{\mathrm{F}-\mathrm{C}}=25.3 \mathrm{~Hz}\right), 134.8,133.8$ $(\mathrm{m}), 131.5\left(\mathrm{t},{ }^{4} J_{\mathrm{F}-\mathrm{C}}=3.0 \mathrm{~Hz}\right), 129.0,117.3\left(\mathrm{t},{ }^{1} J_{\mathrm{F}-\mathrm{C}}=287.0 \mathrm{~Hz}\right) ;{ }^{19} \mathrm{~F}$ NMR (acetone-d $\left.\mathrm{d}_{6}\right): \delta=-$ 104.36 (s, 2F); MS (ESI-MeOH): $m / z=235.1\left(\mathrm{M}^{-}\right), 267.0$. 


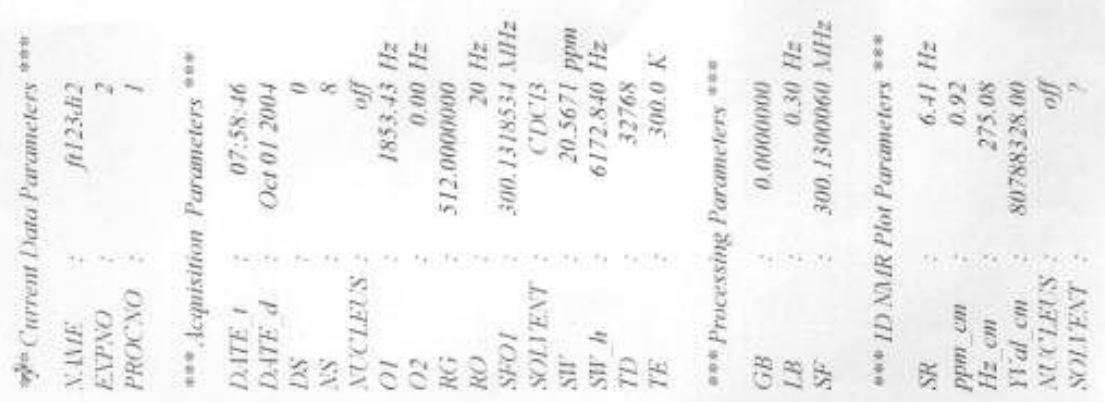

$\overbrace{\frac{1}{4}}^{3}$ thist

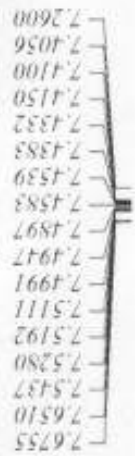
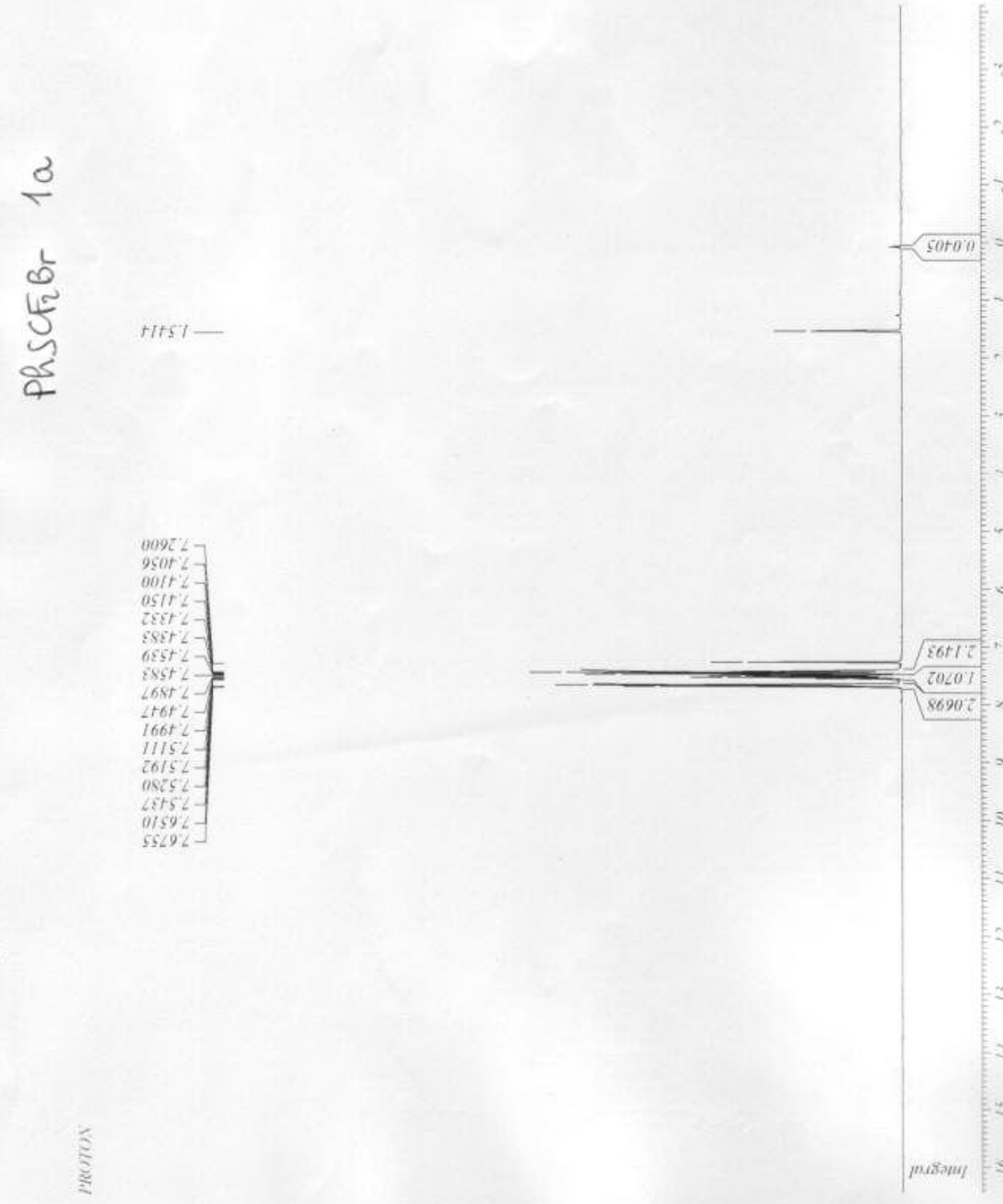


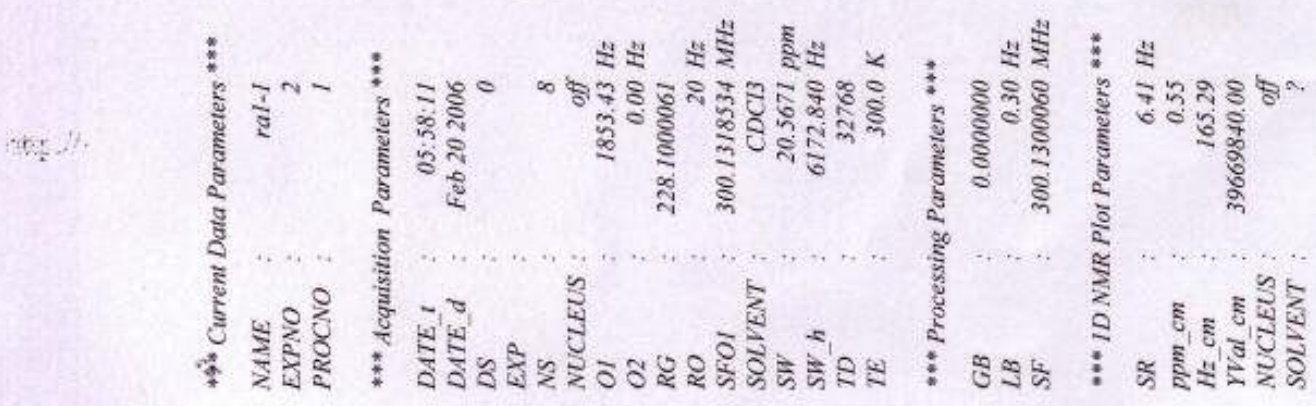
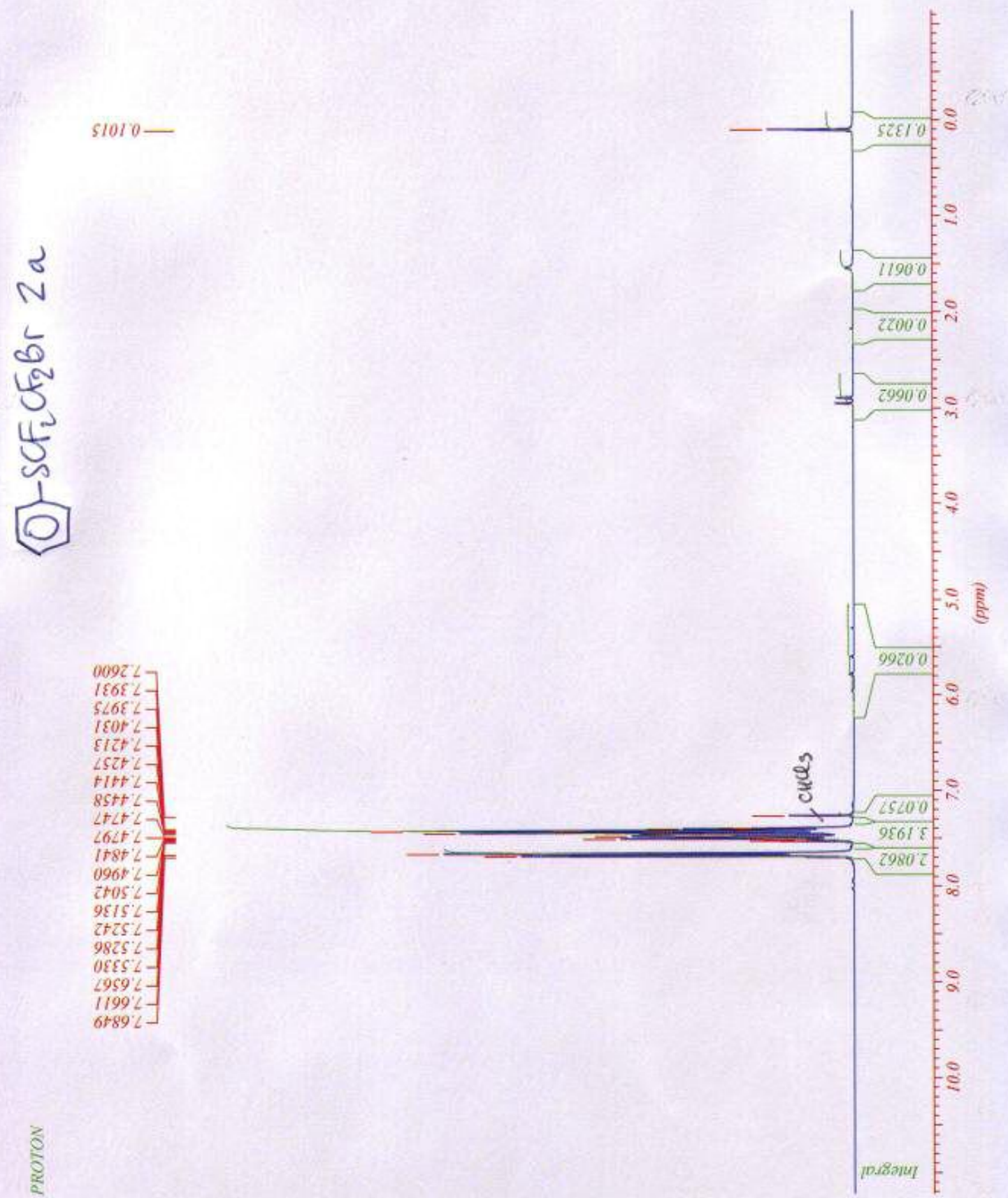

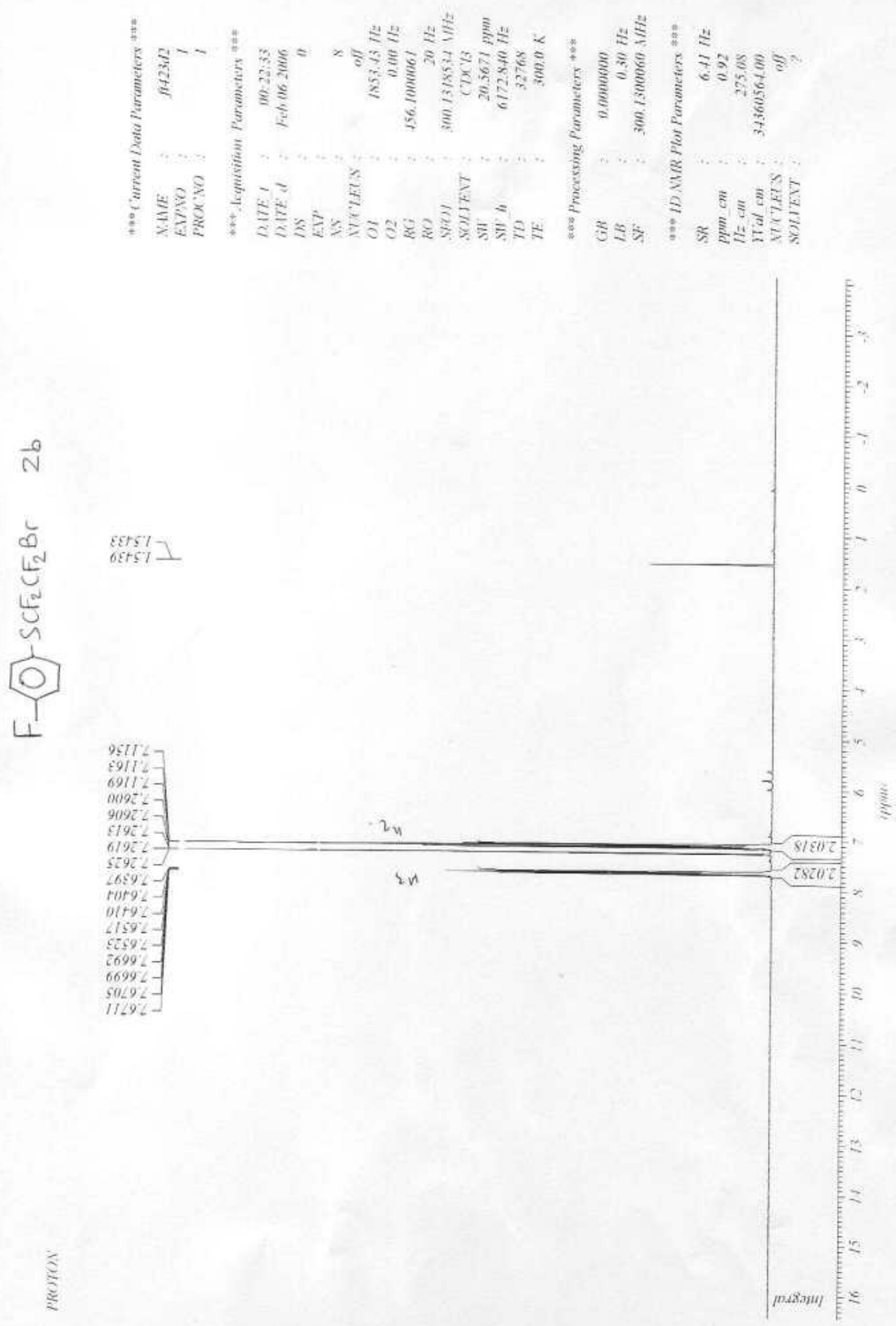


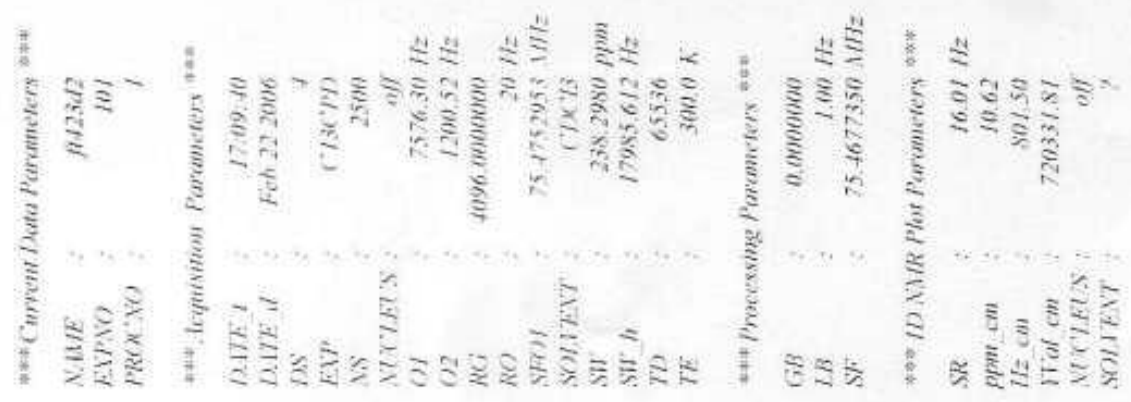

$\sum_{4}^{0}$

009122

tze-9Il

coles 211

9080 $211-$

$1228.811]$

eflosil]

$9126871-$

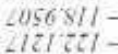

8865651
$+20265]$

58t1 $891-$

it $6+991-$

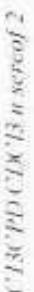

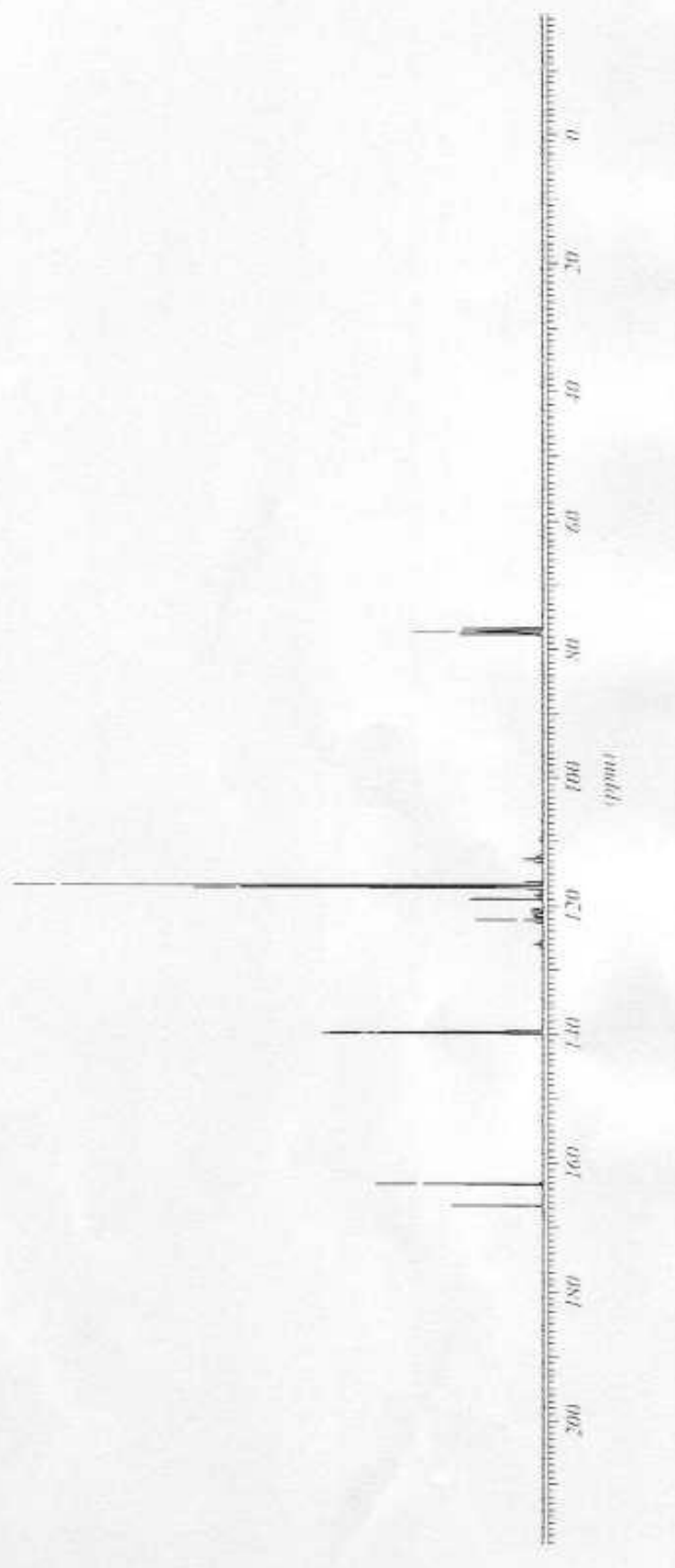



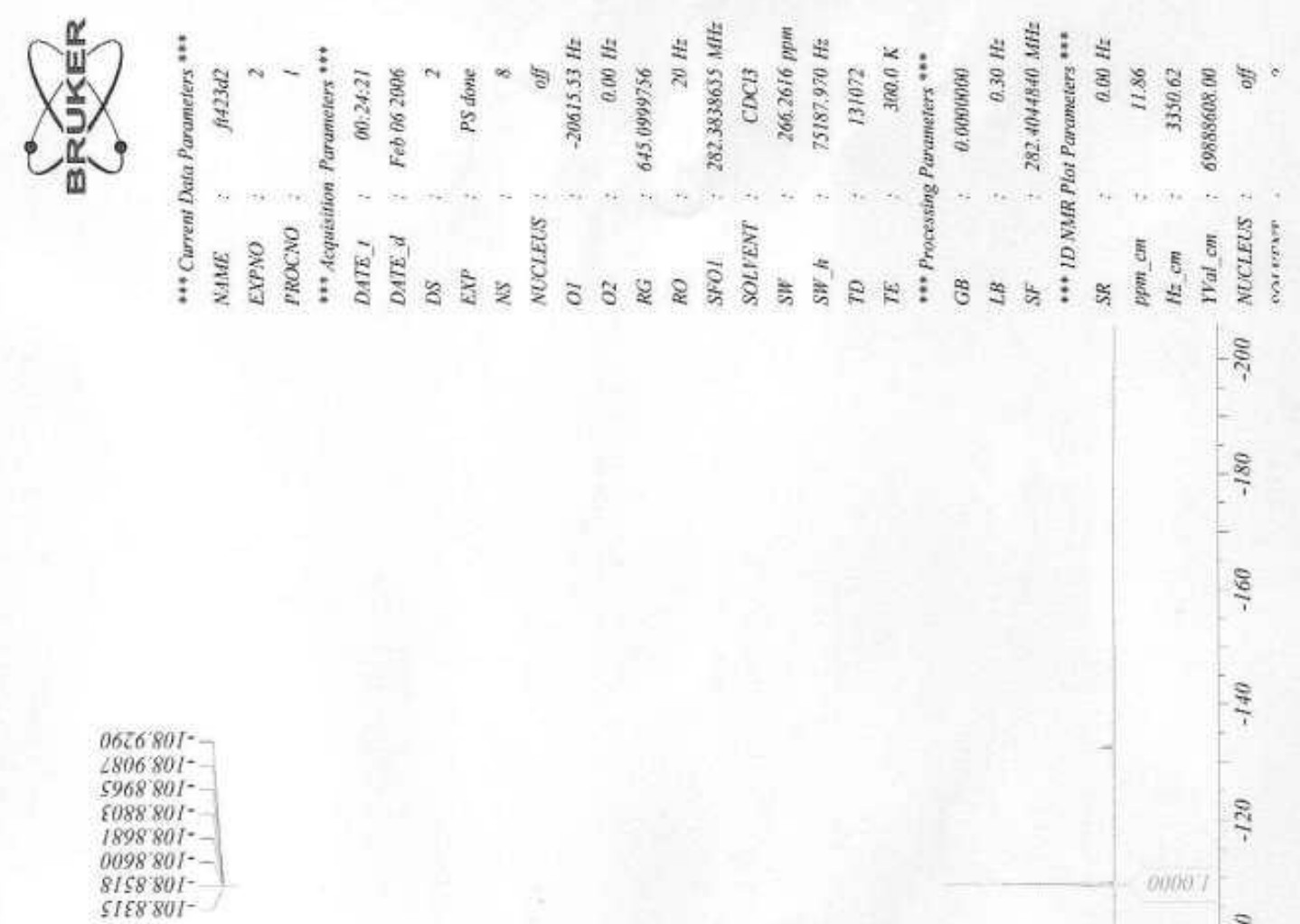

$685098-$

f020 98-

$026658^{\circ}$

$12+209$

$\angle 81229^{\circ}$

$258929-$
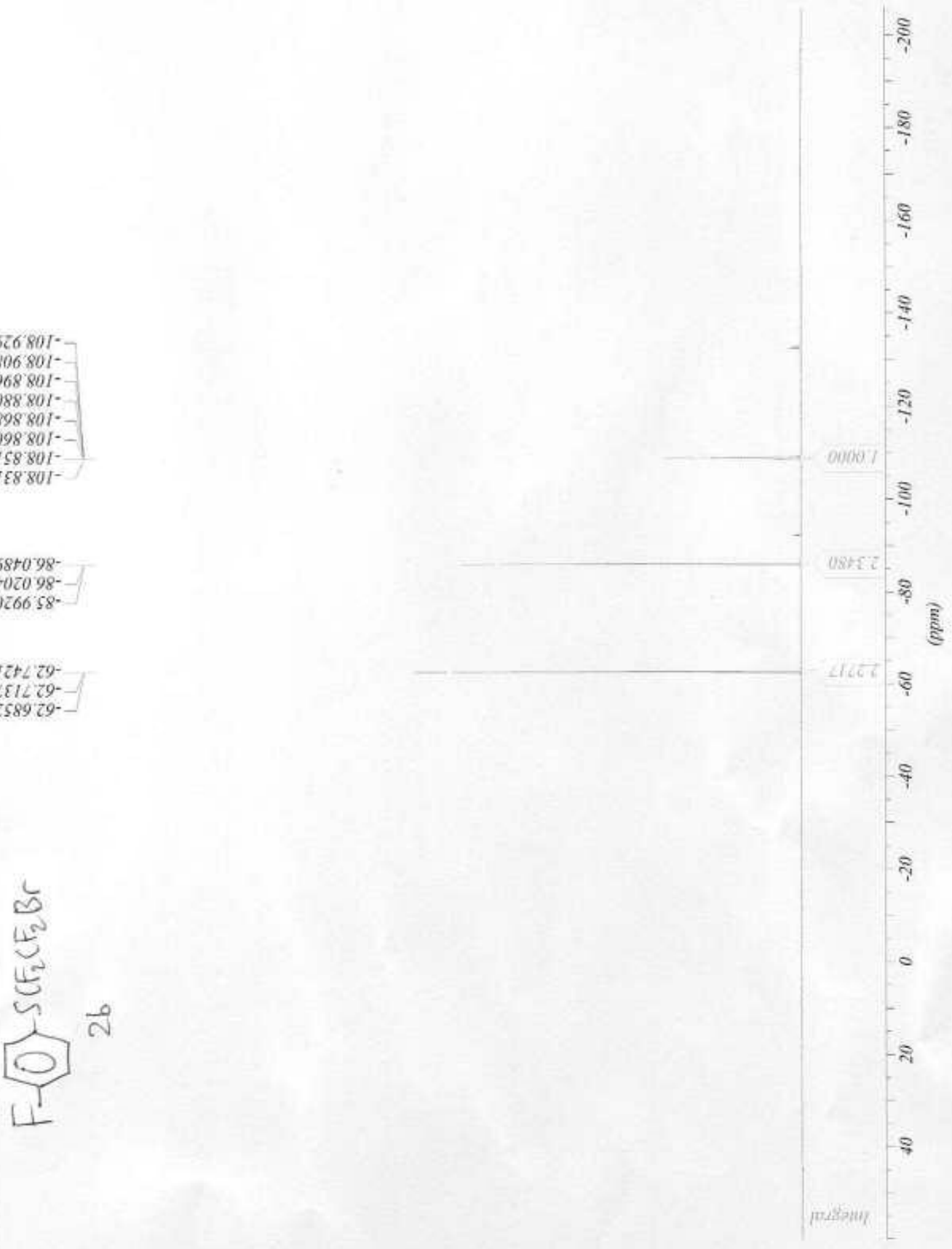

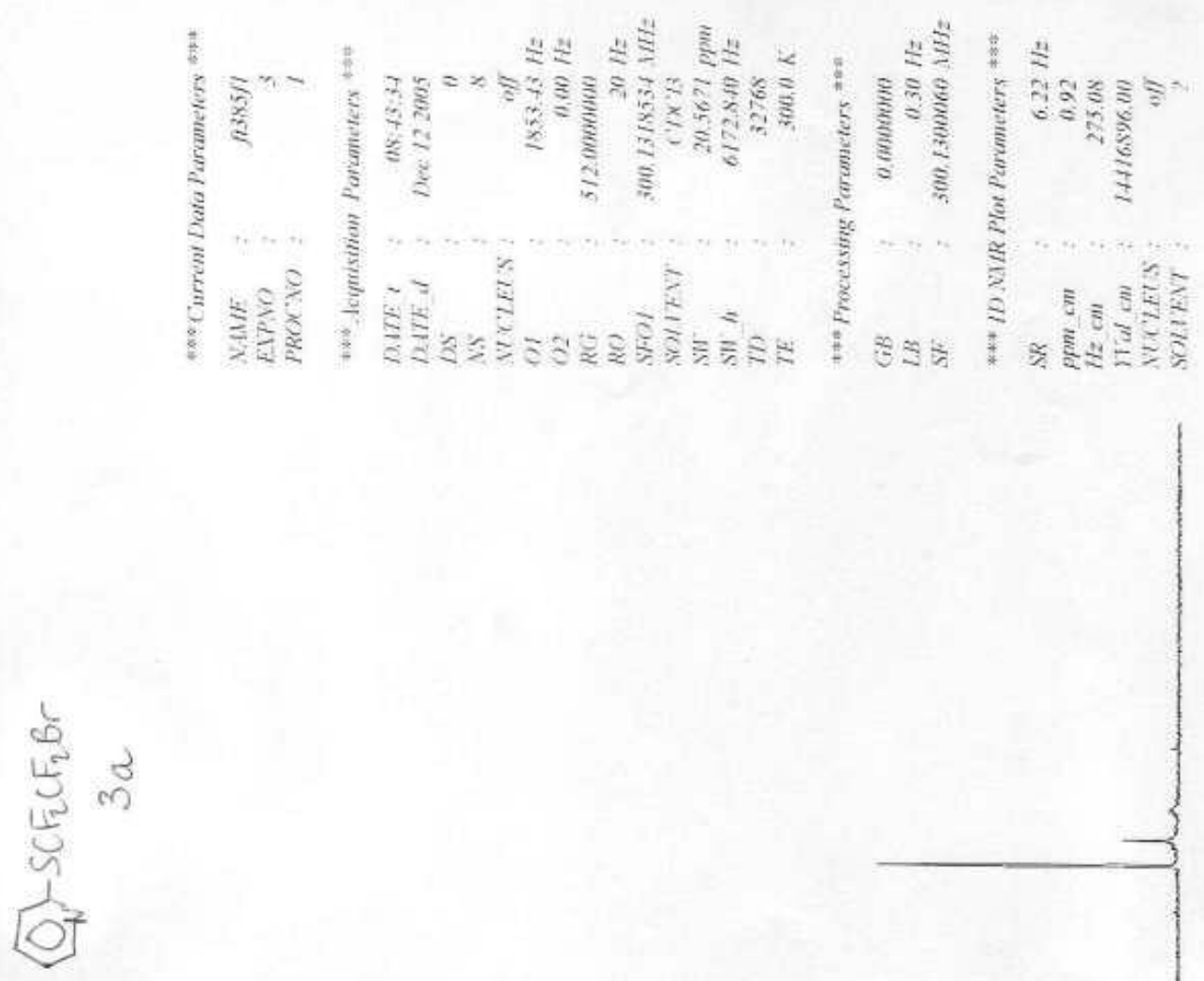

b092:

व1rE

GL5E:

CLSEZ

$+592^{\circ}-2$

722:-

$5509^{\circ}$

2169:

HIL -1

onc.2

68222

is.

SILLC

$82+98$

60598

$18598-$
$601998-$

1+998-

$24998-$

$66998-$

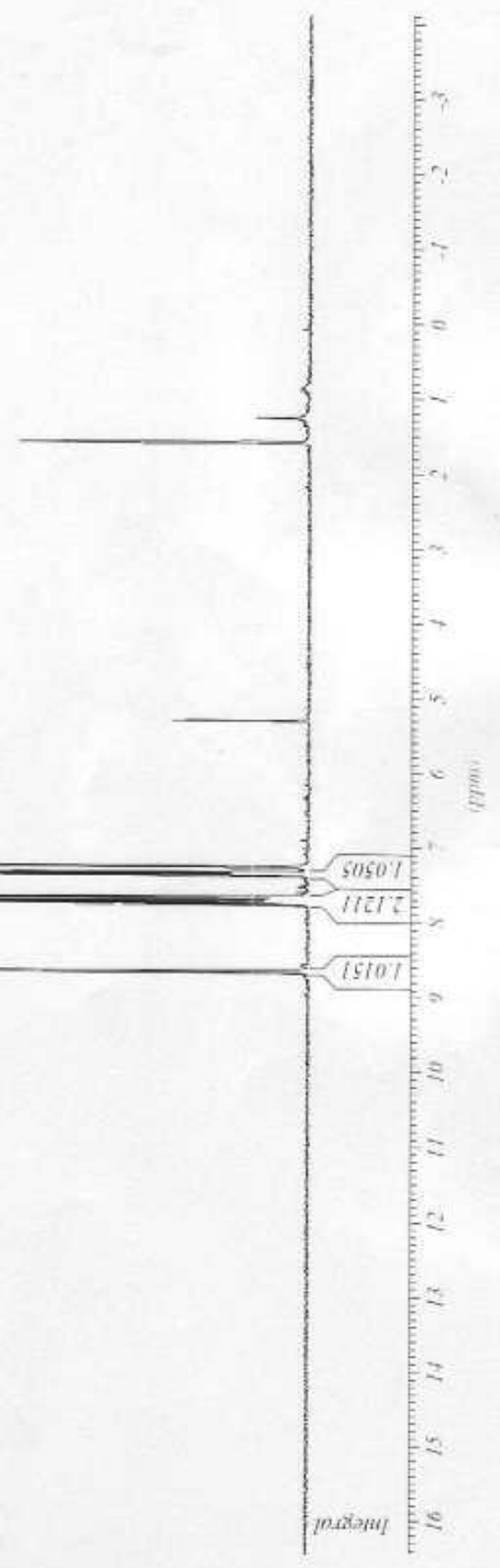



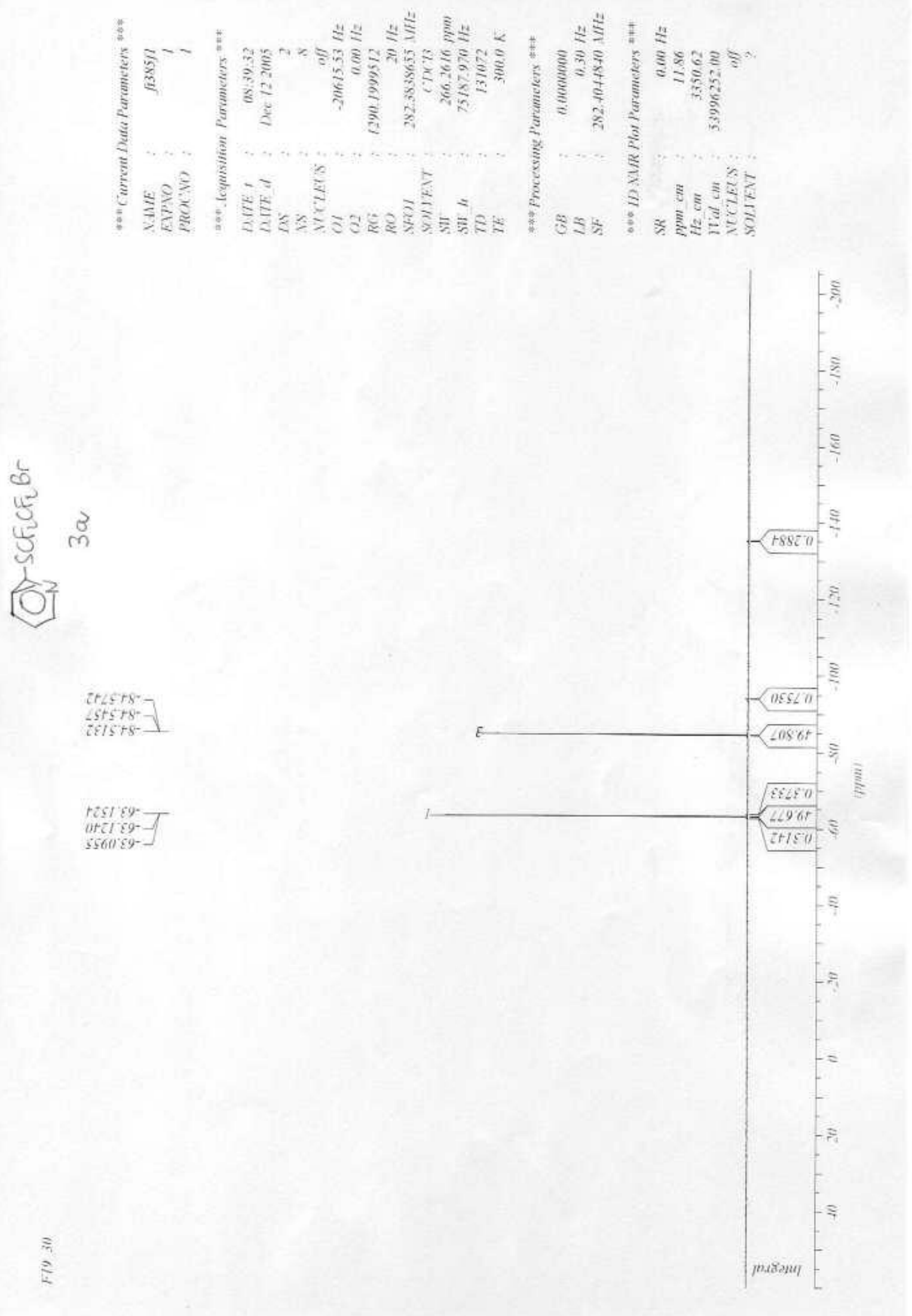


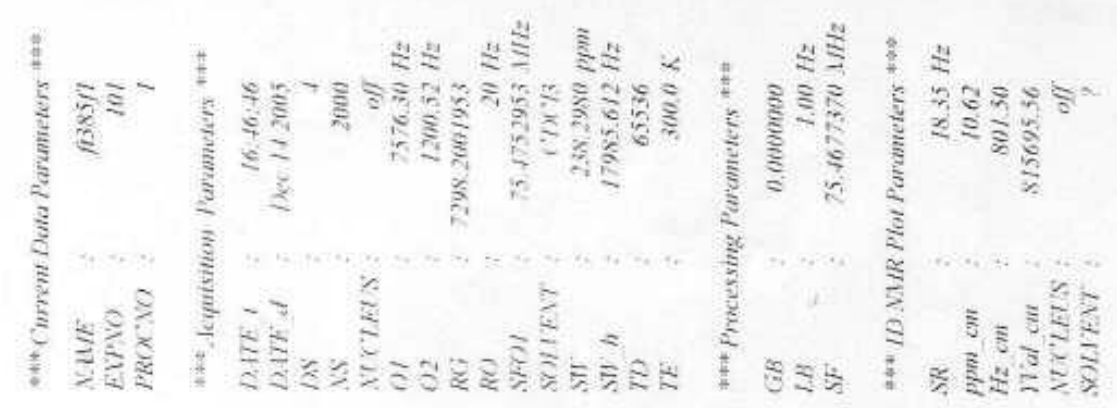

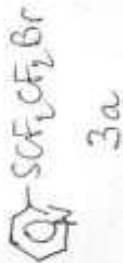

6ues:92-

sise 202

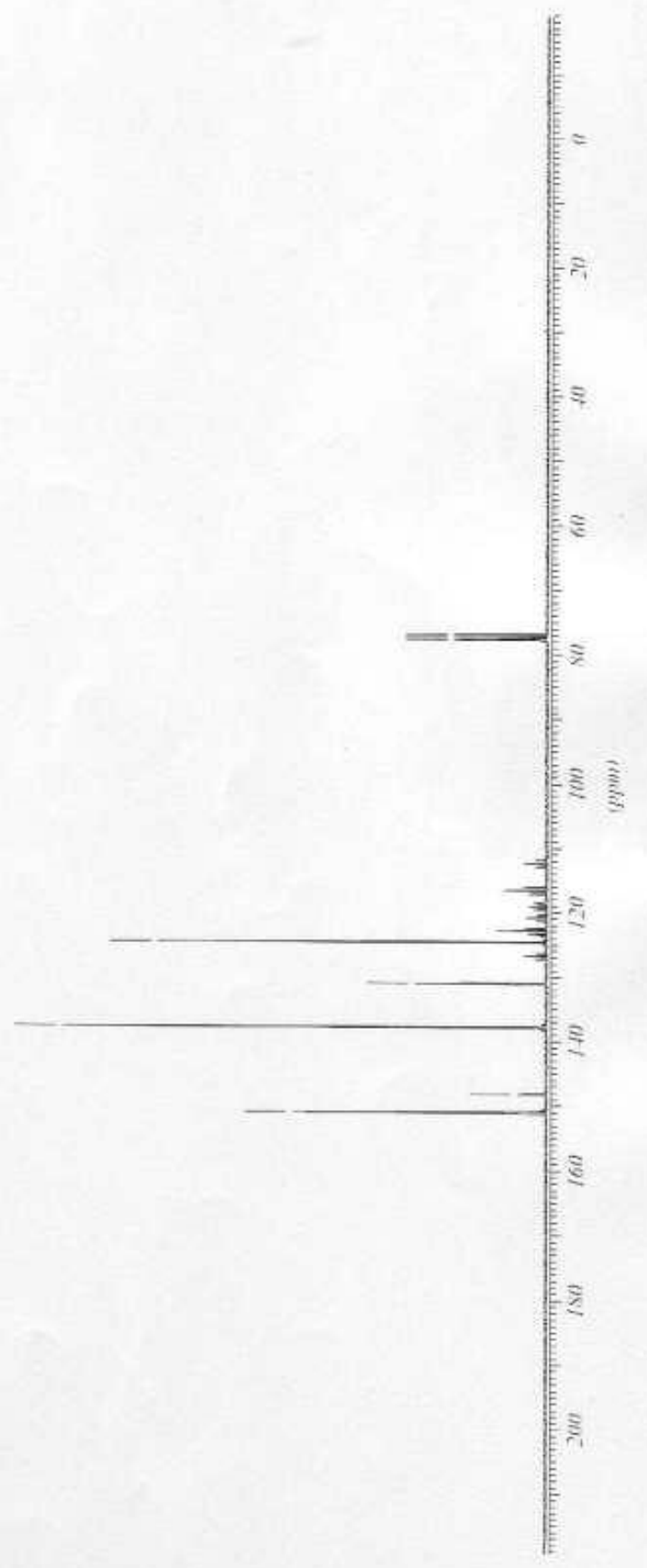

$9295+21-$

c6+6?

aSE 4 \&

6021 SHI -

5826051 - 


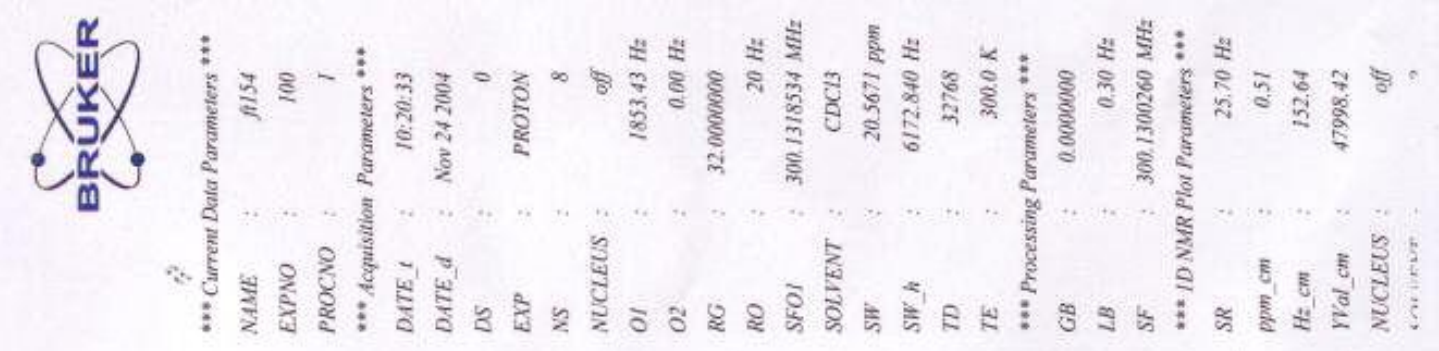

$\angle 8+0^{\circ} 0$

:

8
$\forall$

$\sum_{\substack{n \\ 4}}^{m}$

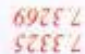

$\angle A F E 2$

stres

$10522-$

tISSE

SORSL

OR8S

29092

$8119 \mathrm{~L}$

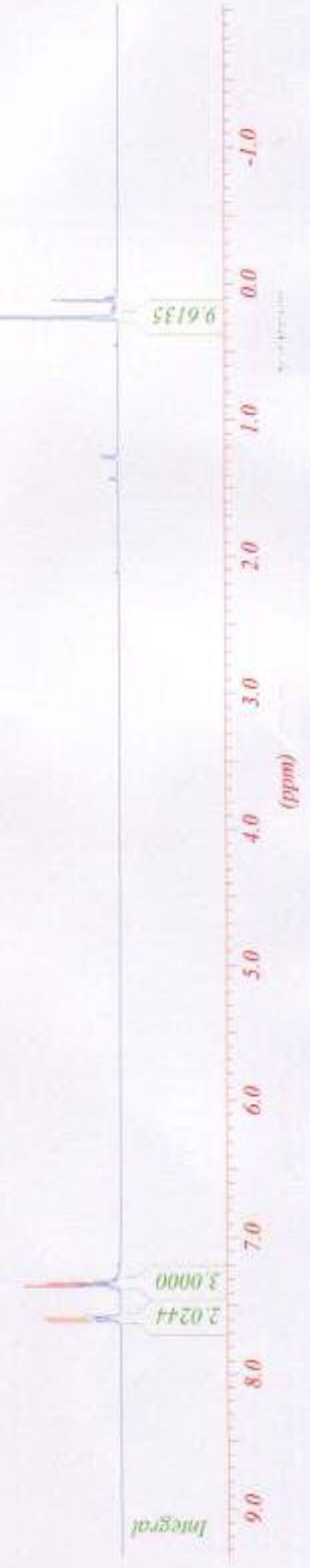




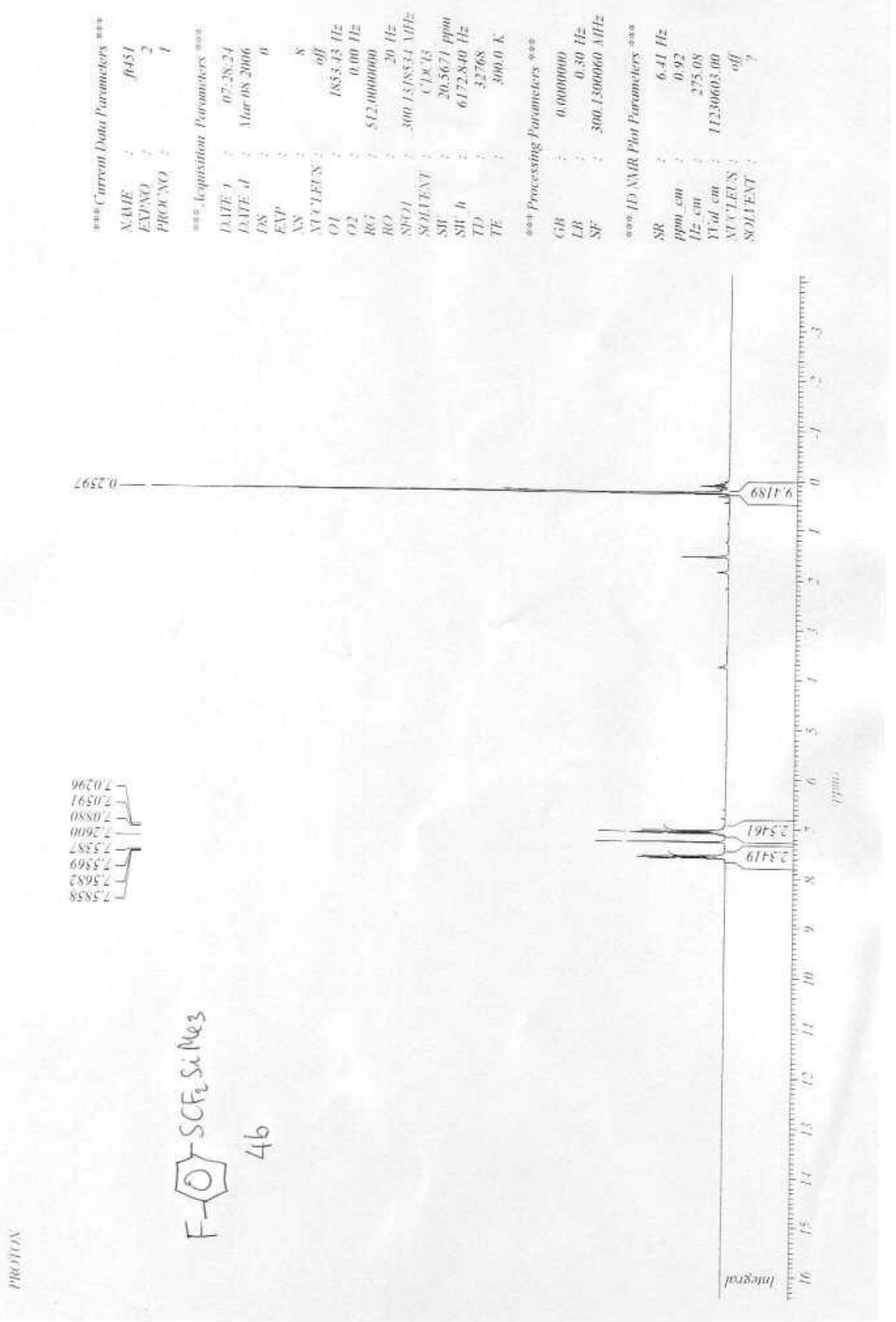




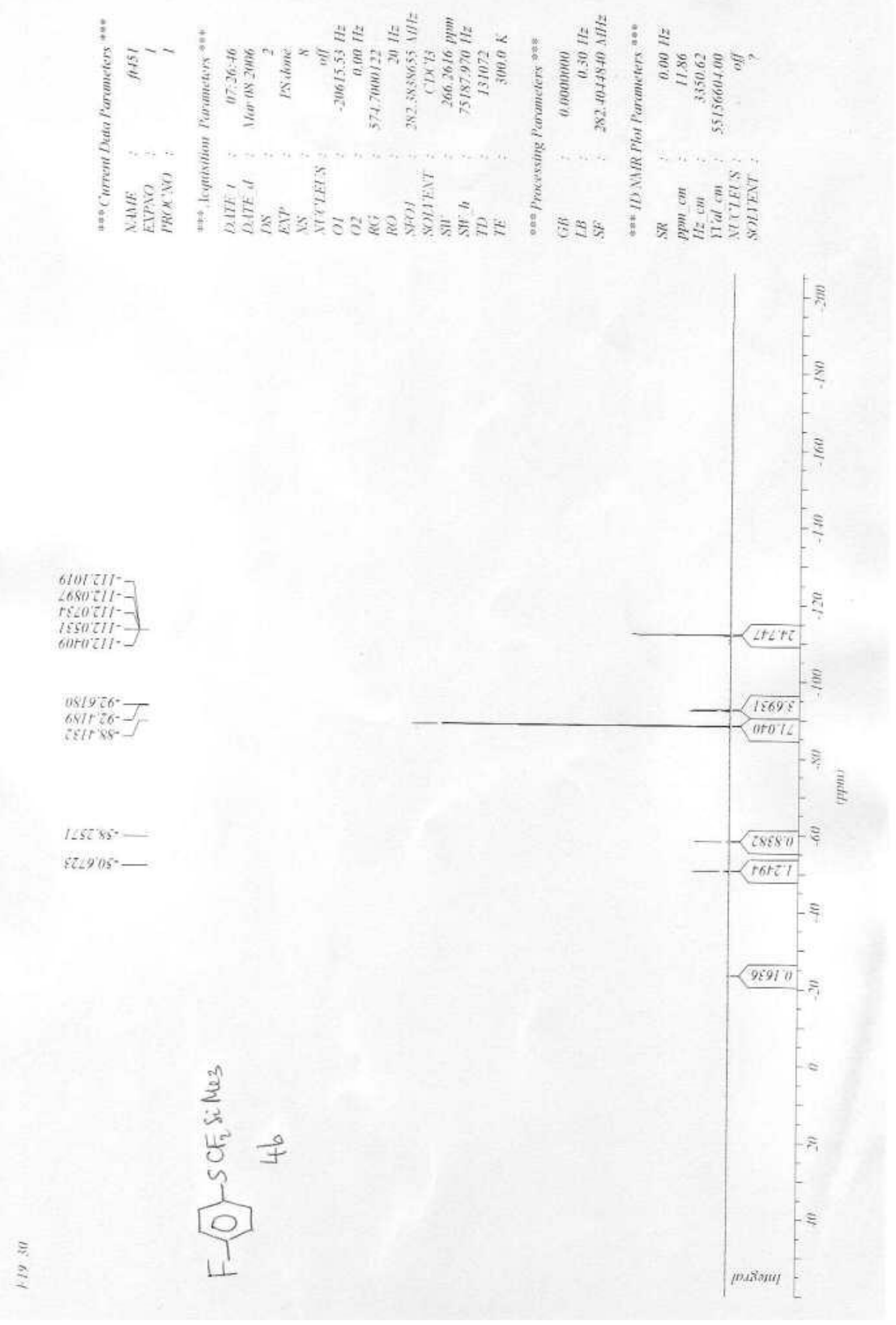



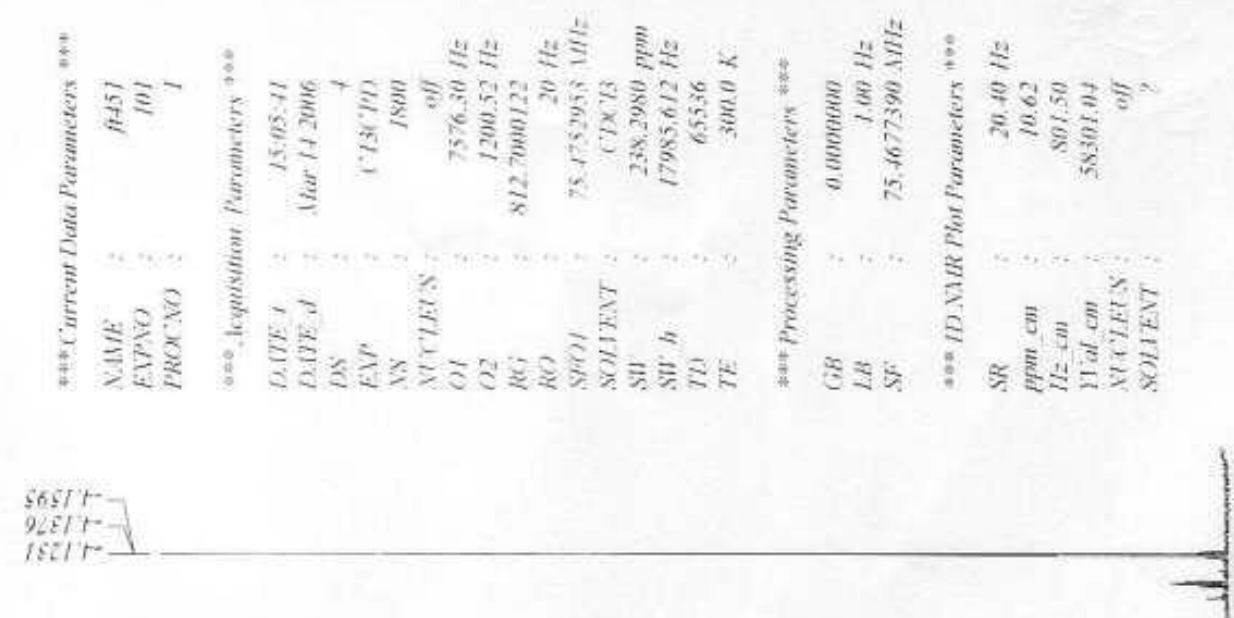

009122

2880211

lsetict -

Listiet -

or 25 हE -

riLtsel

oresel-1

[S6cse]

6892891-

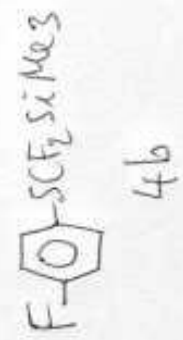



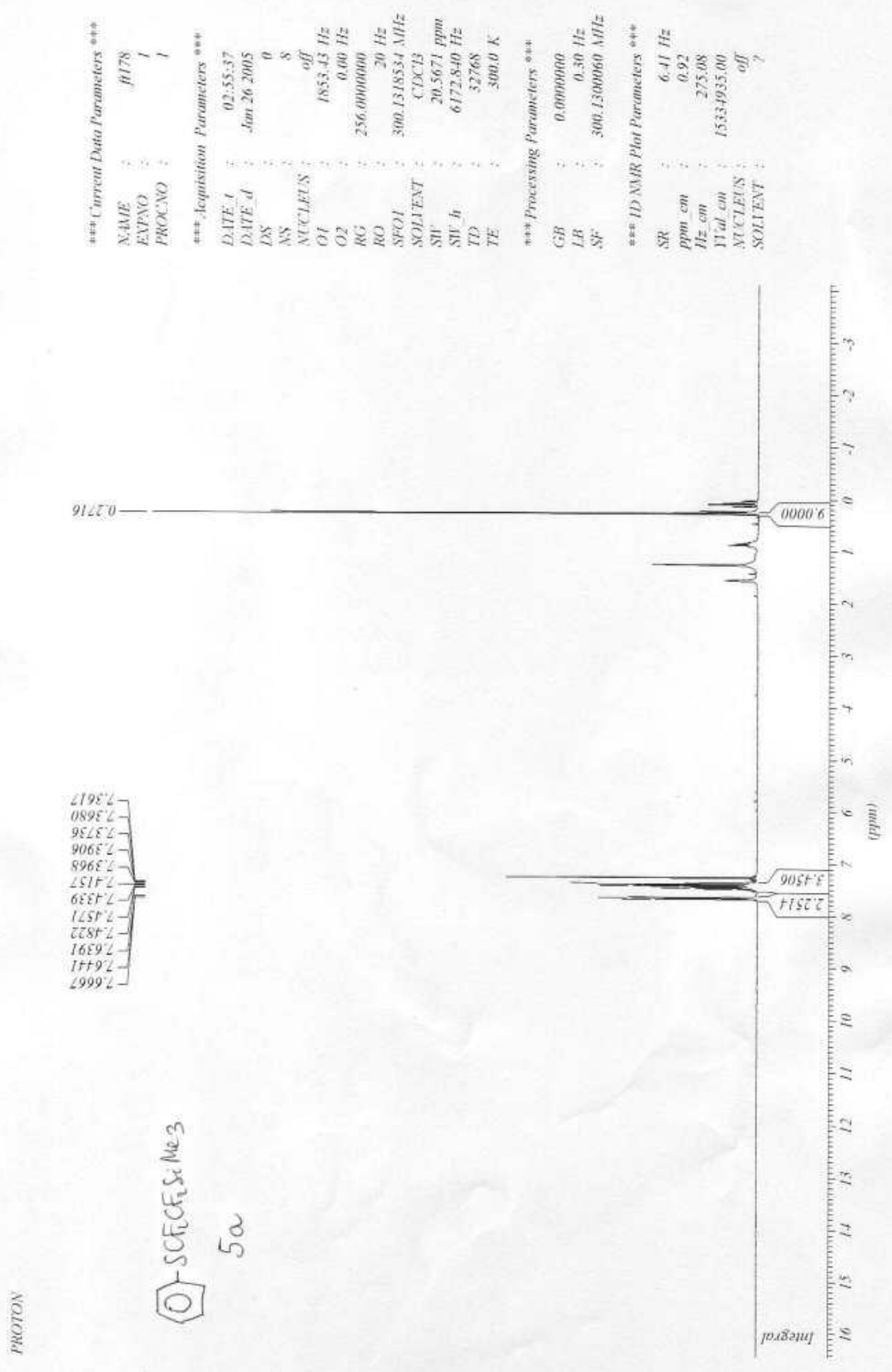

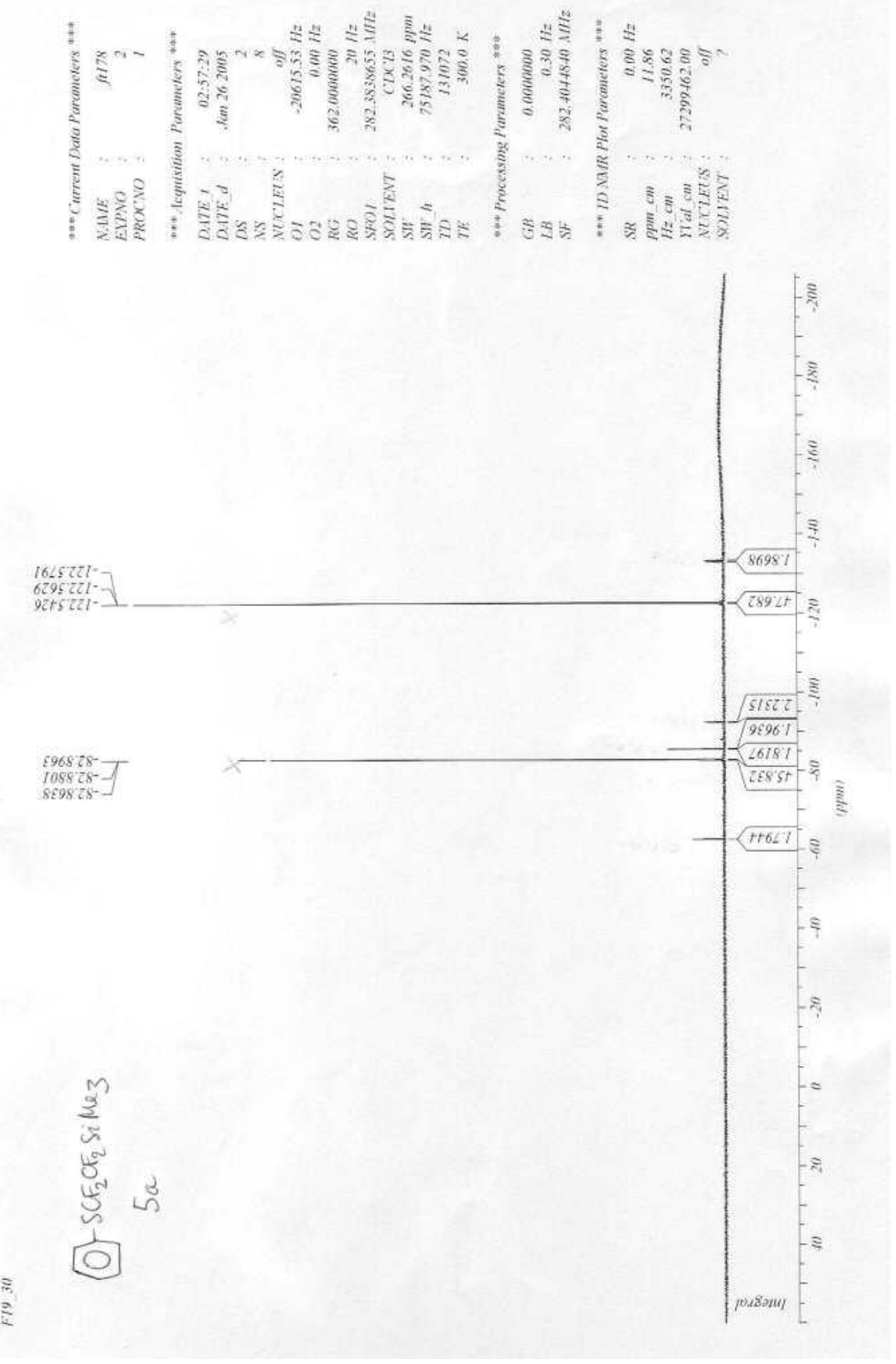

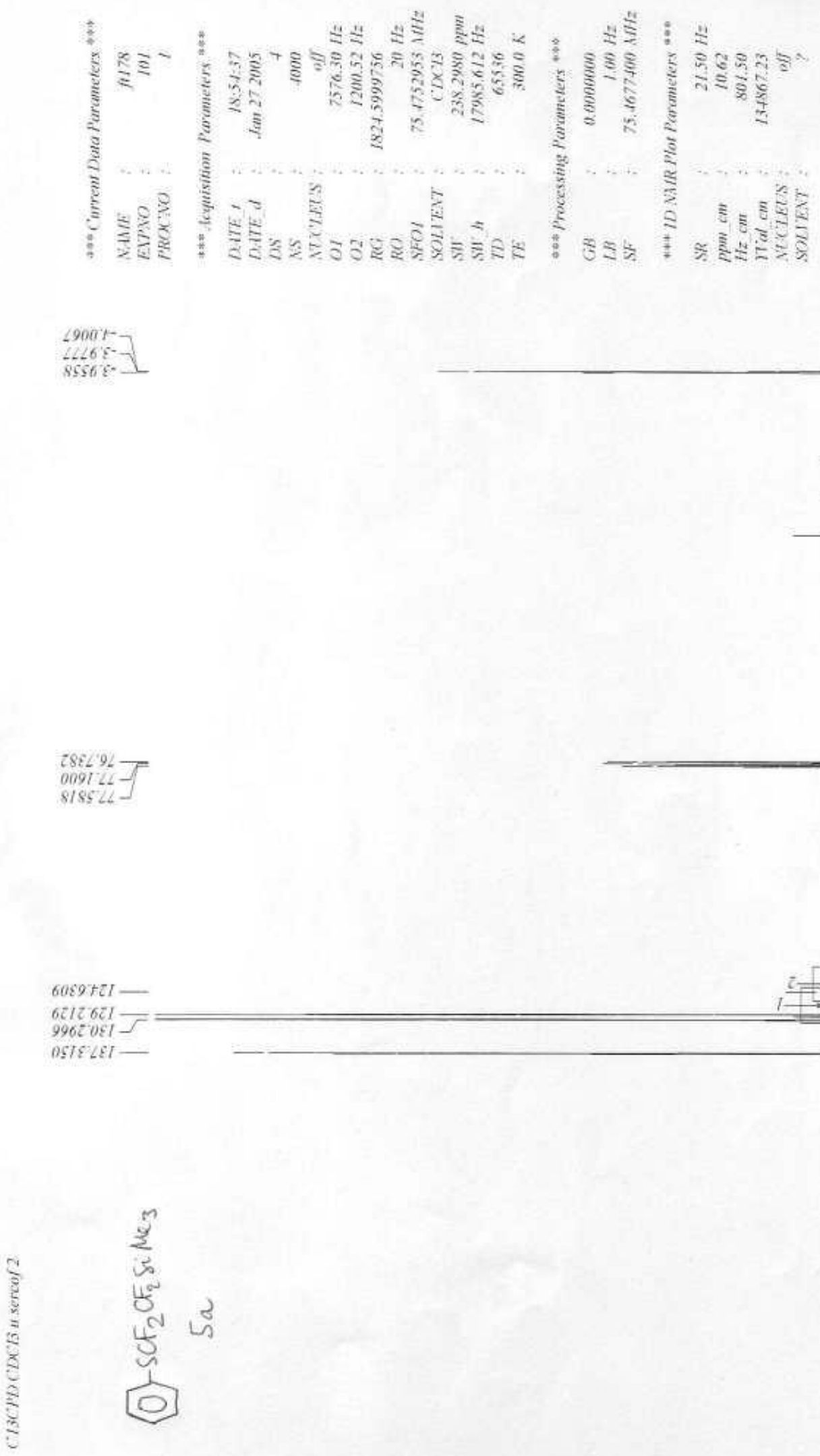


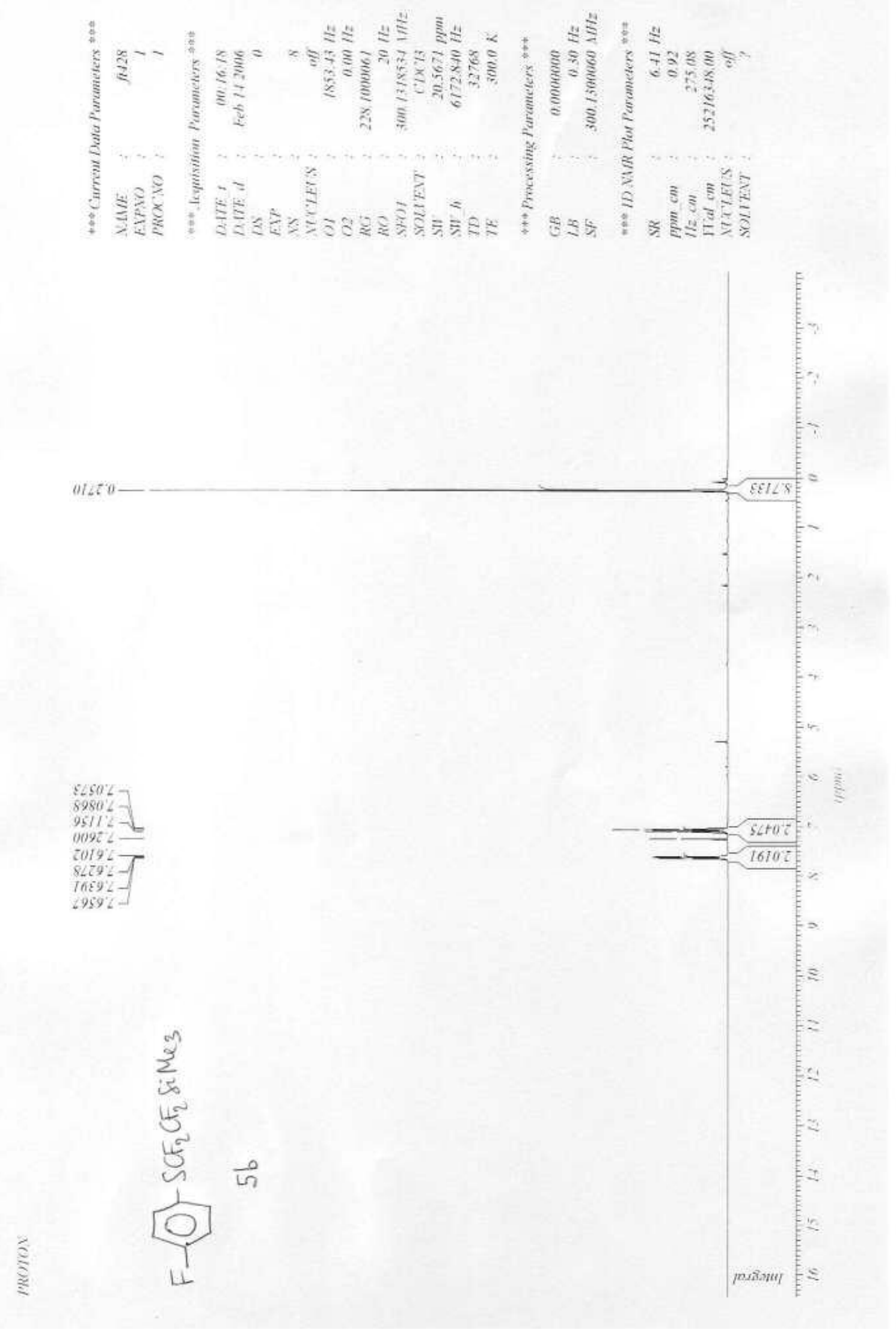




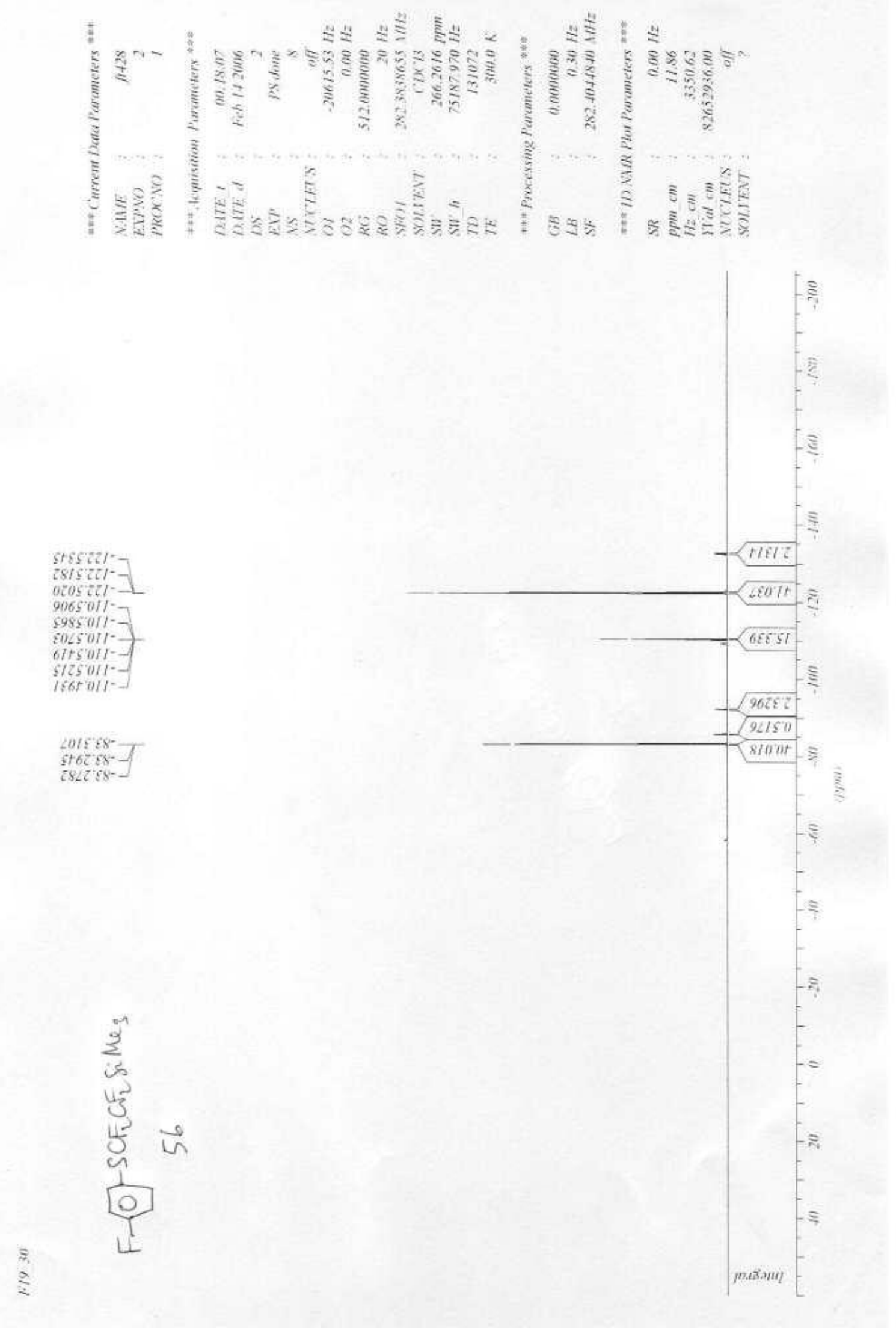



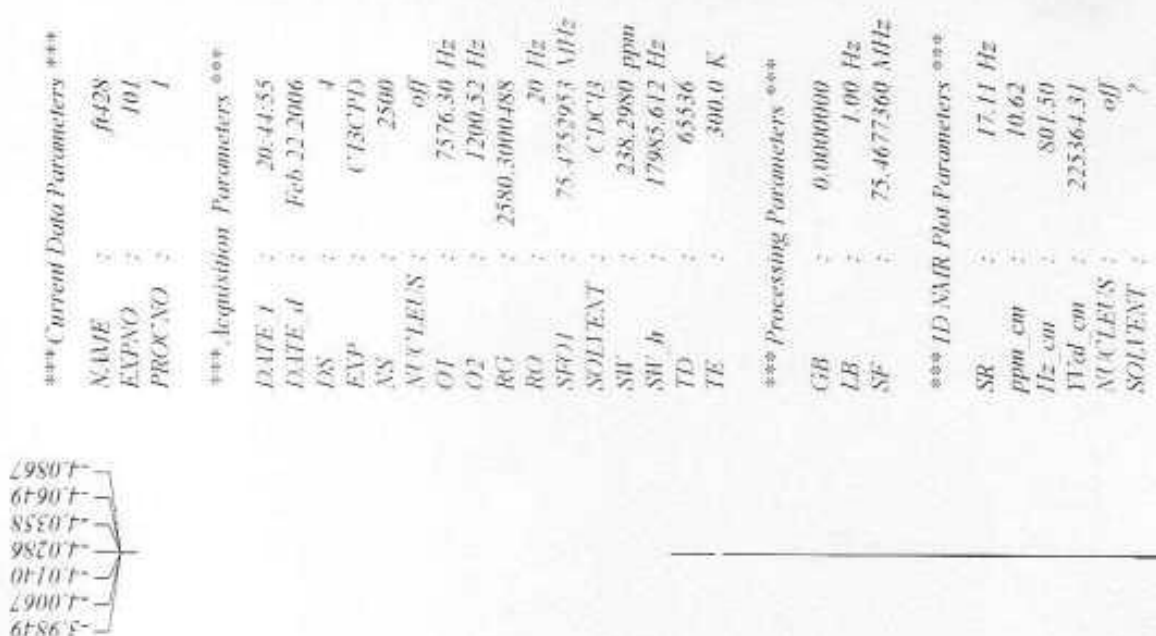

$009122-$
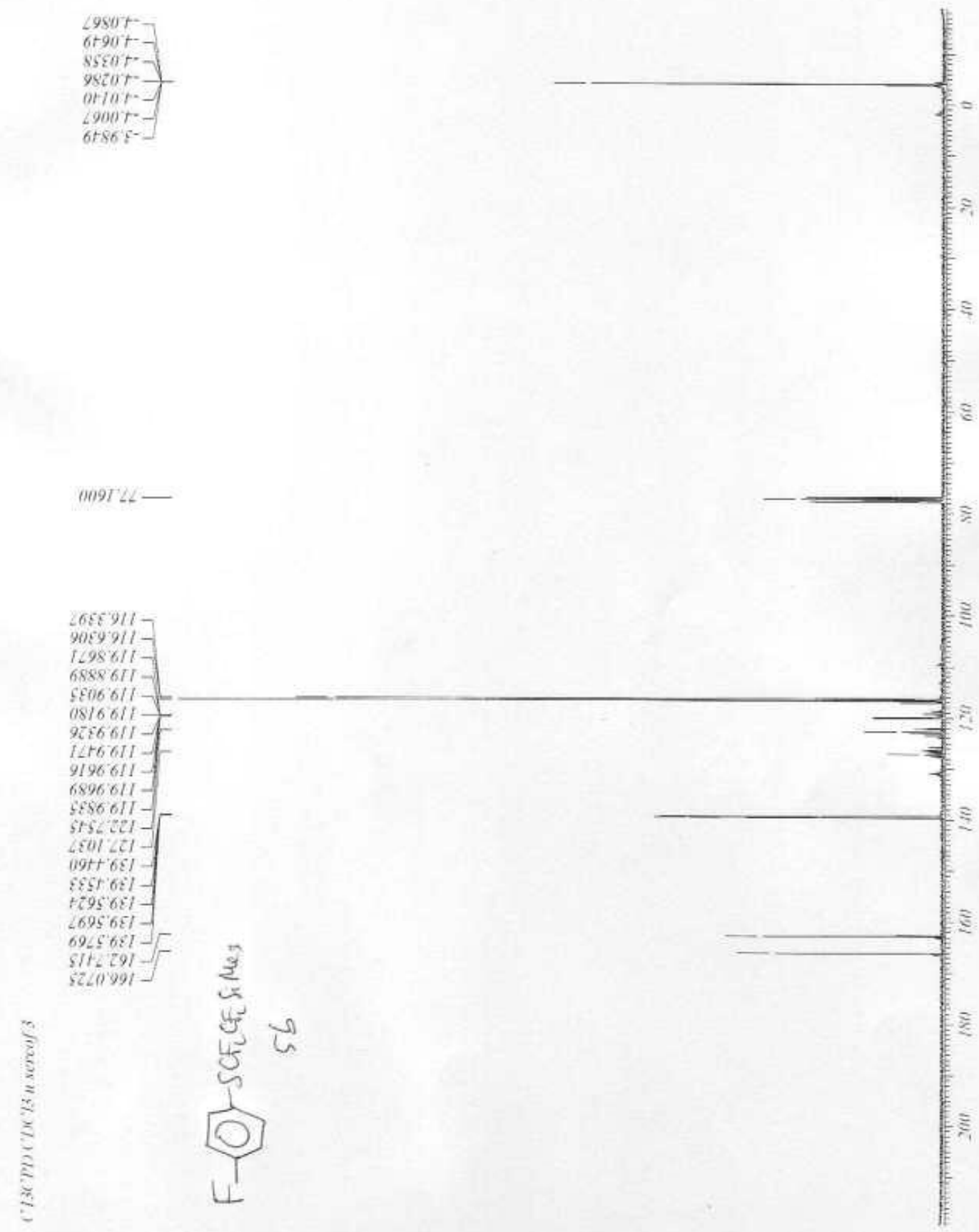


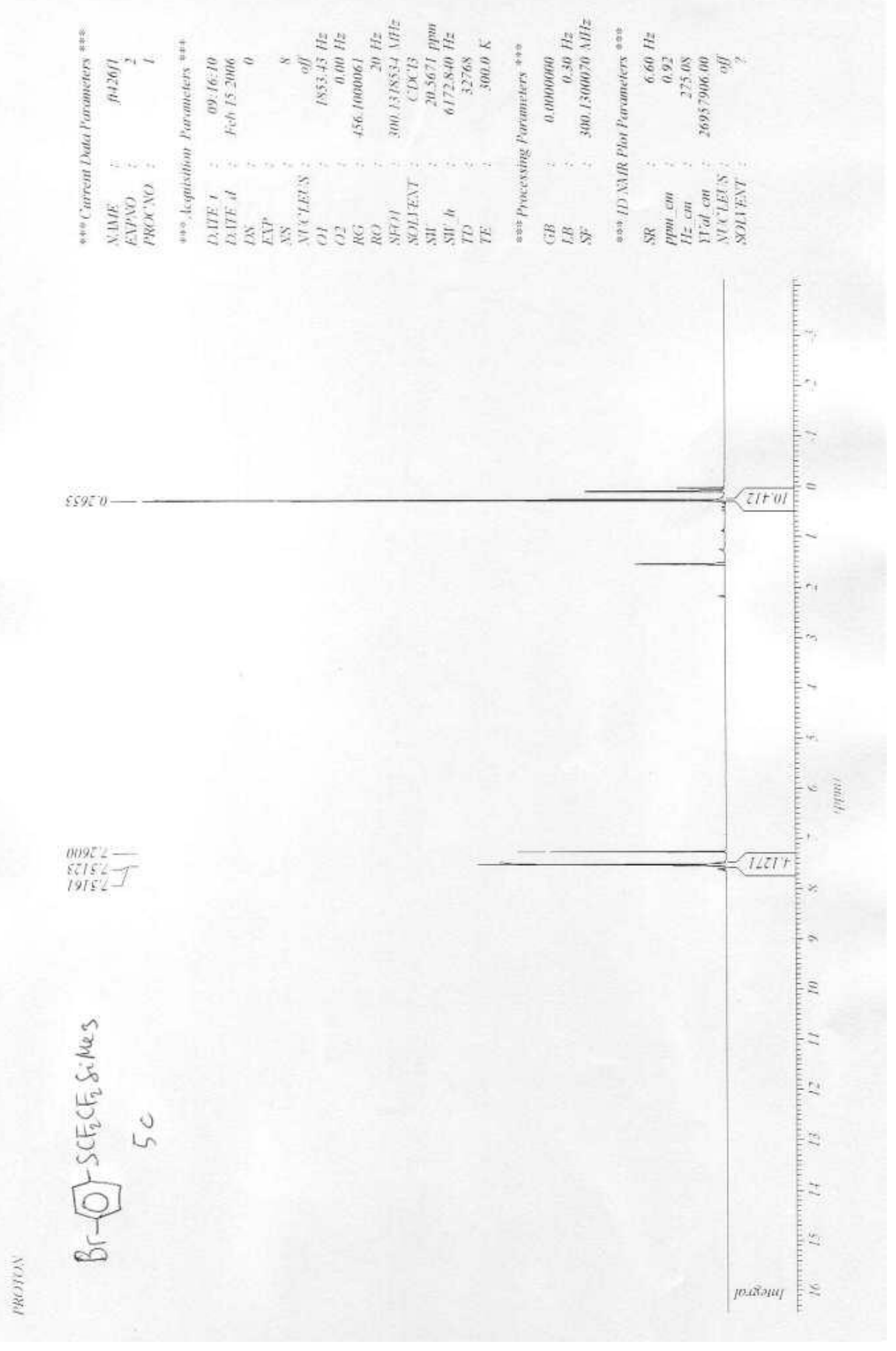



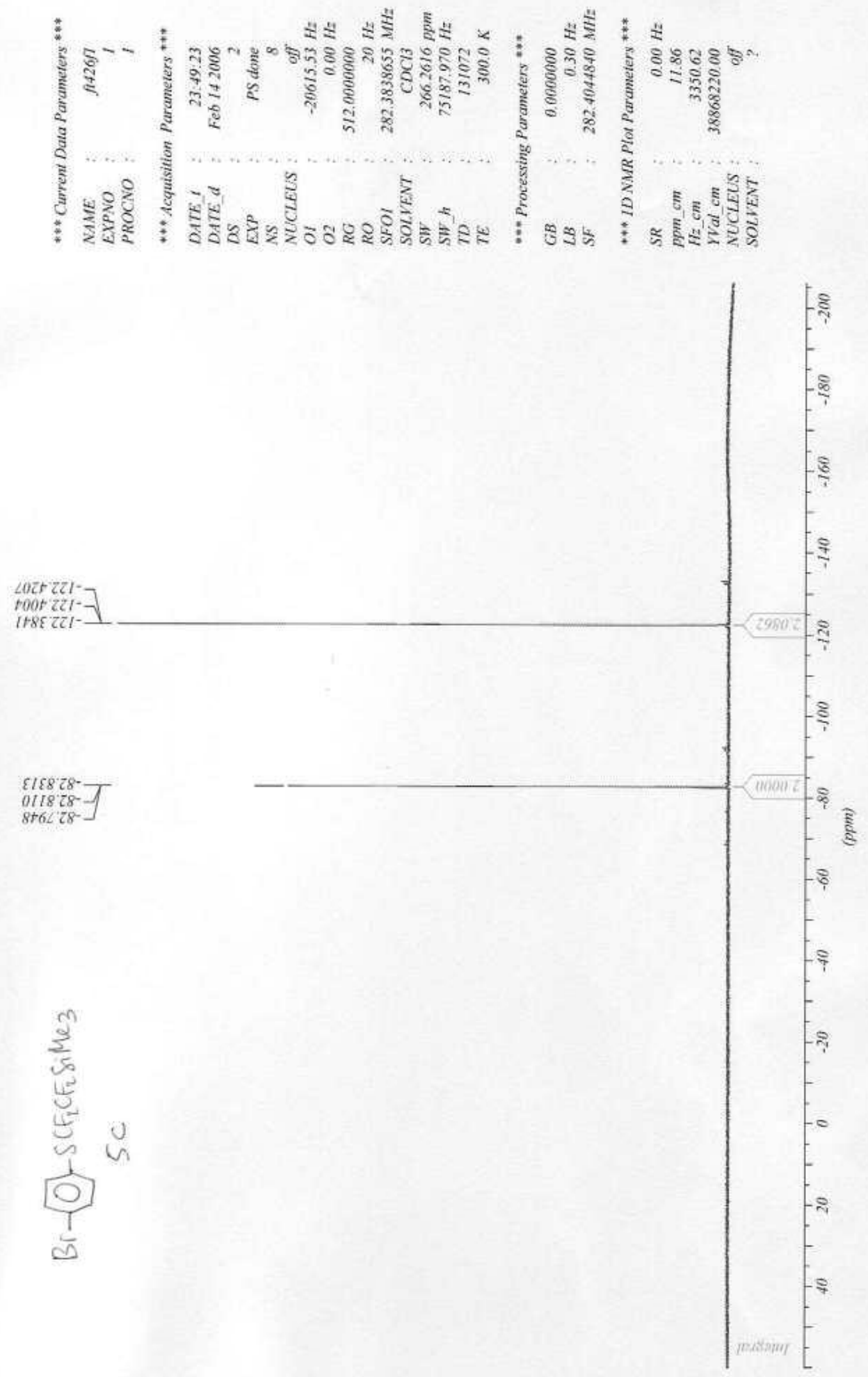


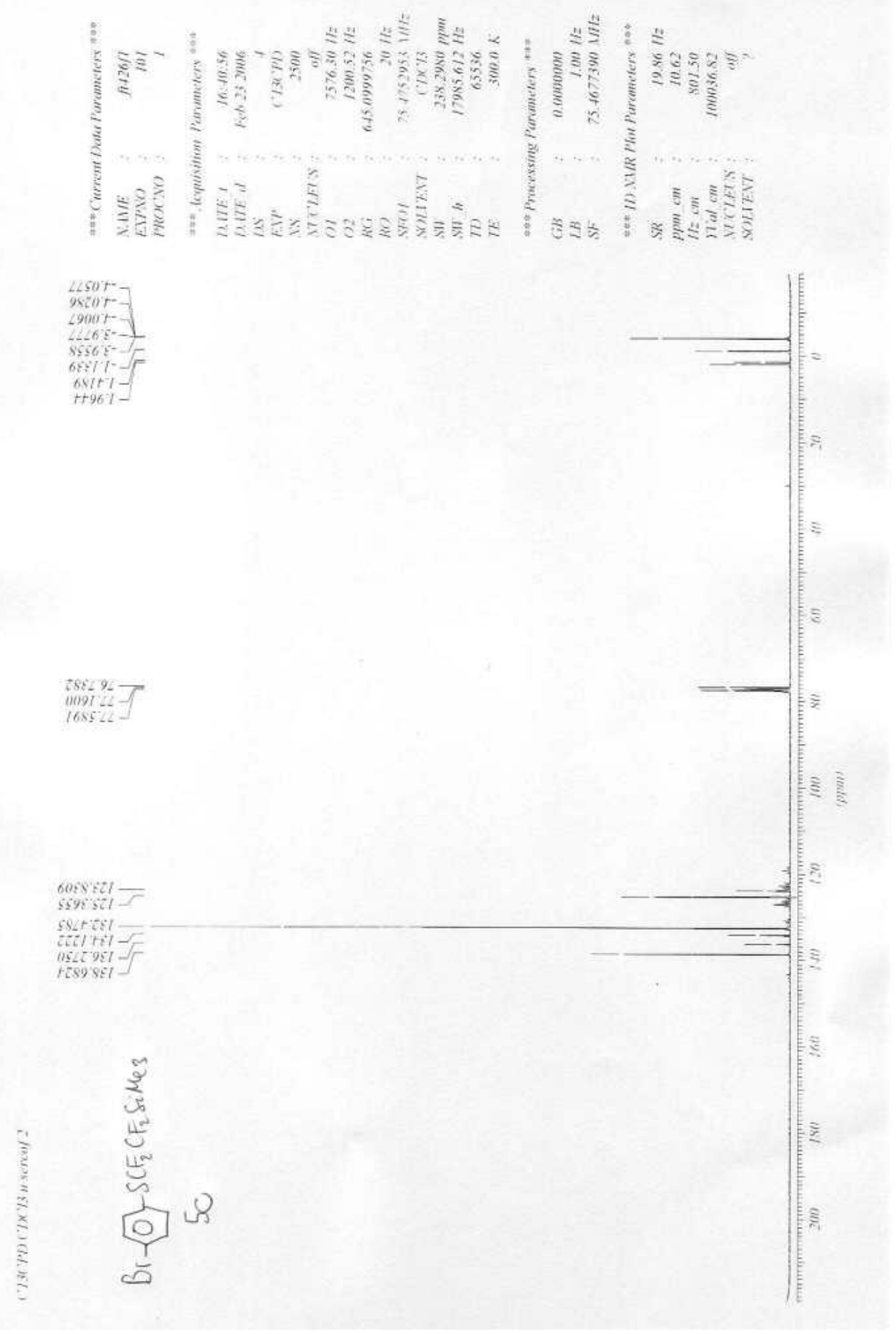




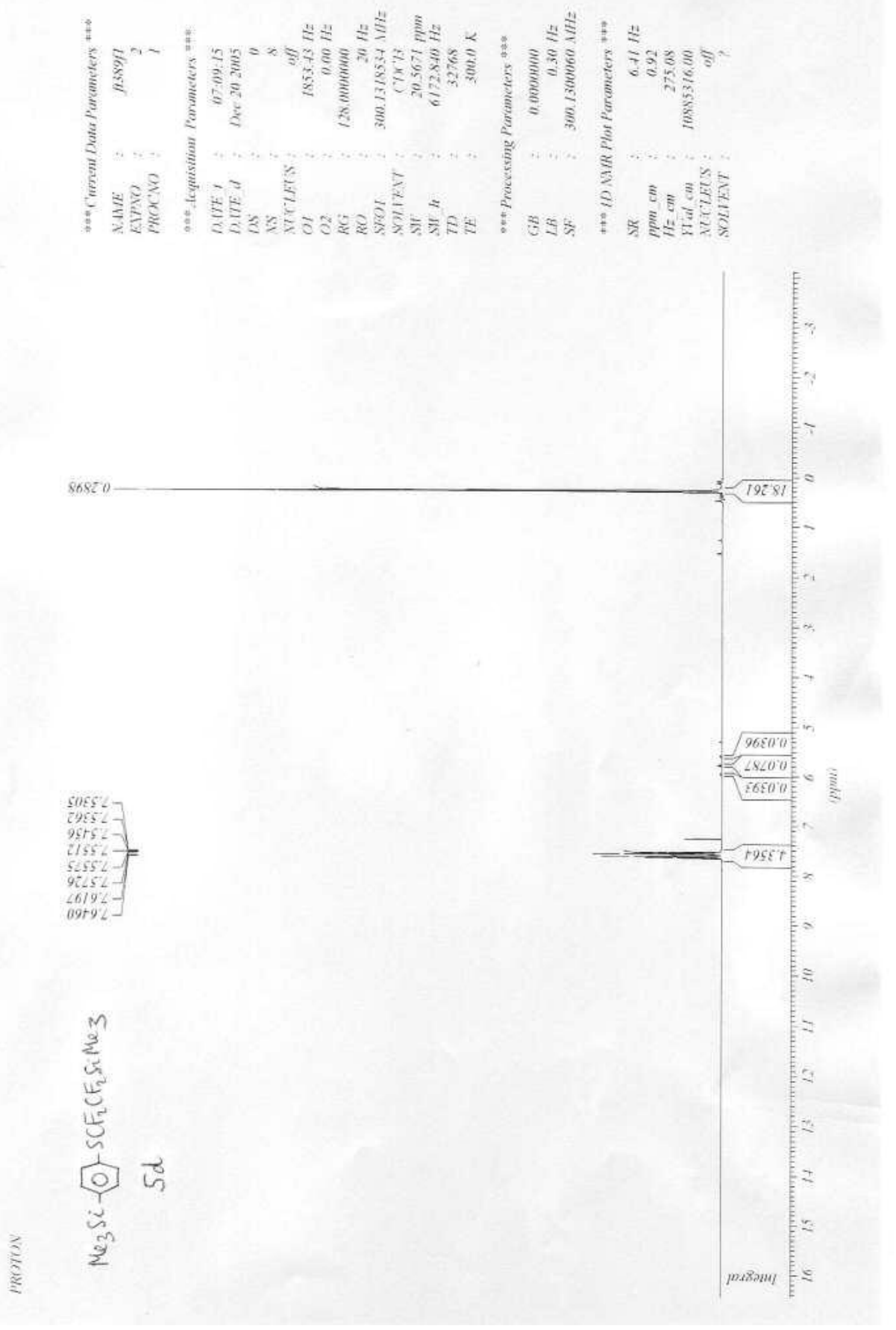



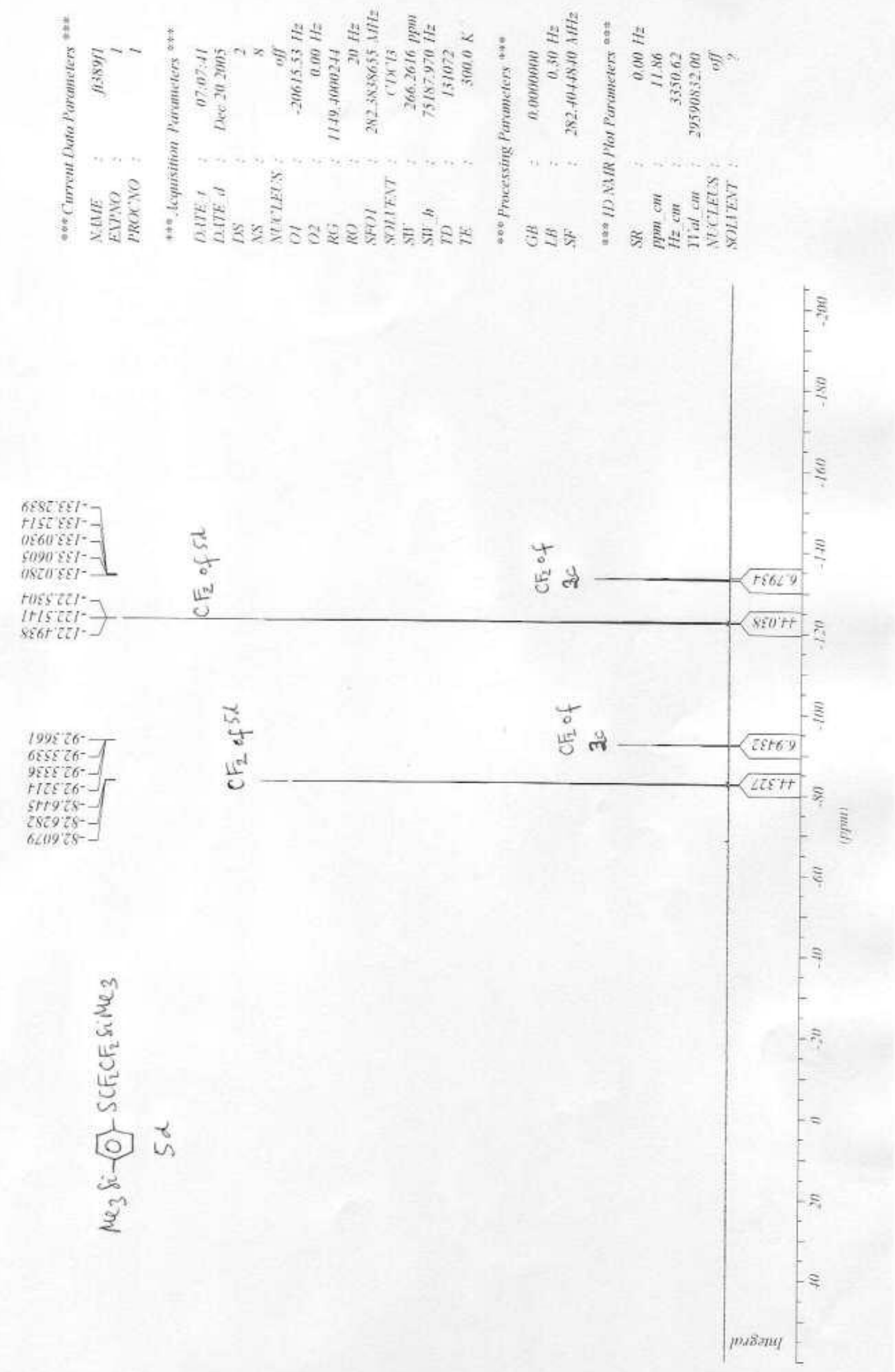


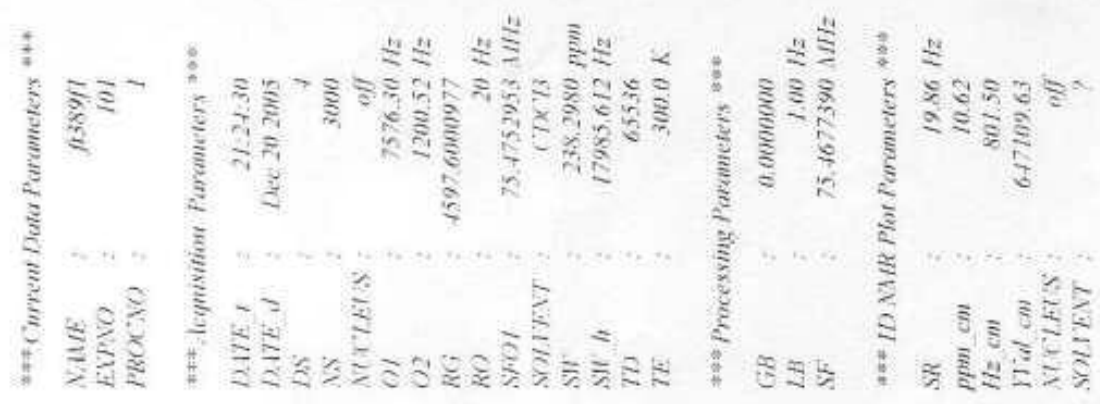

$\frac{1}{4}$

CE66:-

1896 :-

Elto:-

5616 :-

t068\%:-
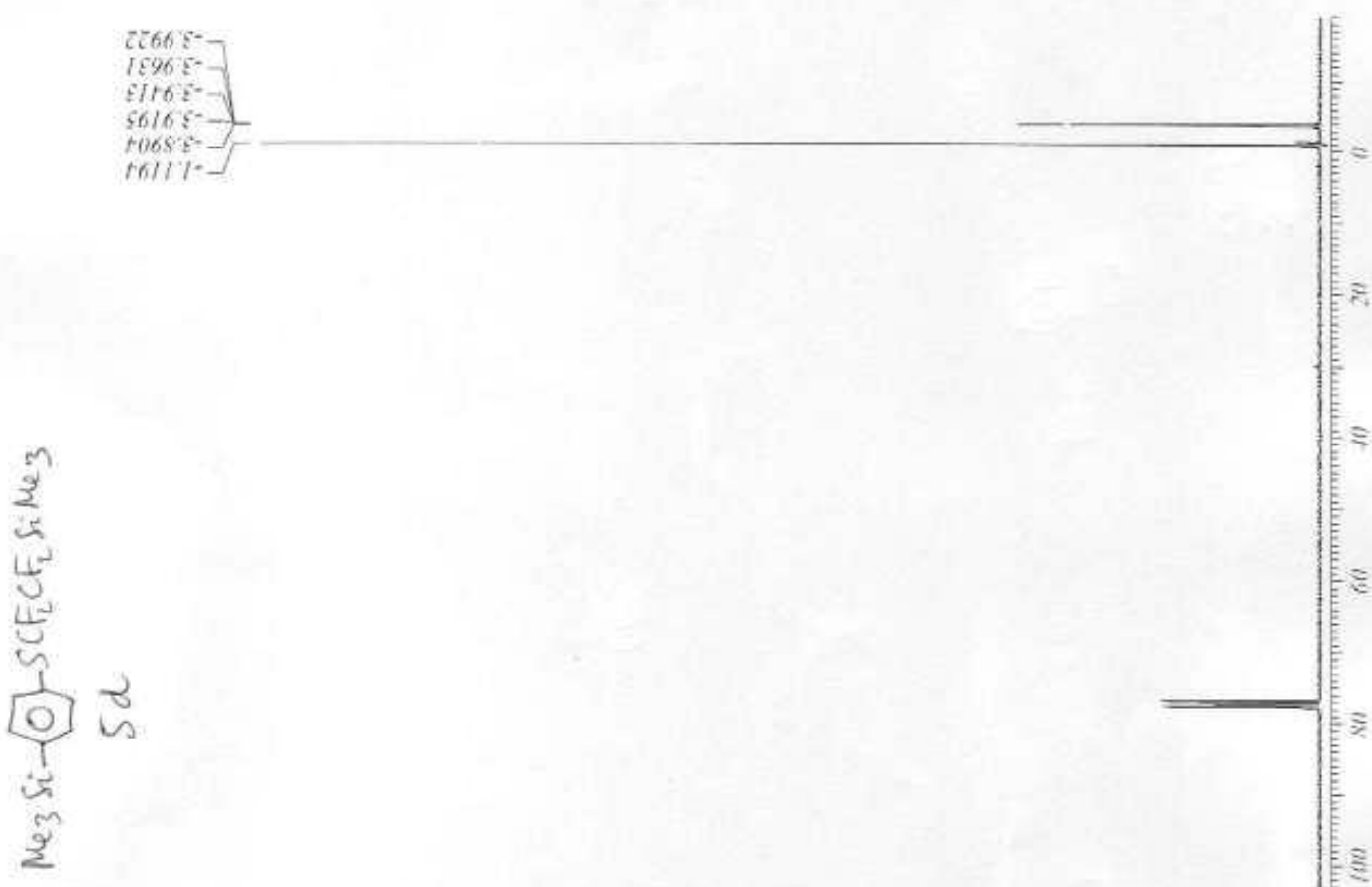

$s s t s t l-$

$5621+E=$

rottel-

EZNE पs I

ESIEET -

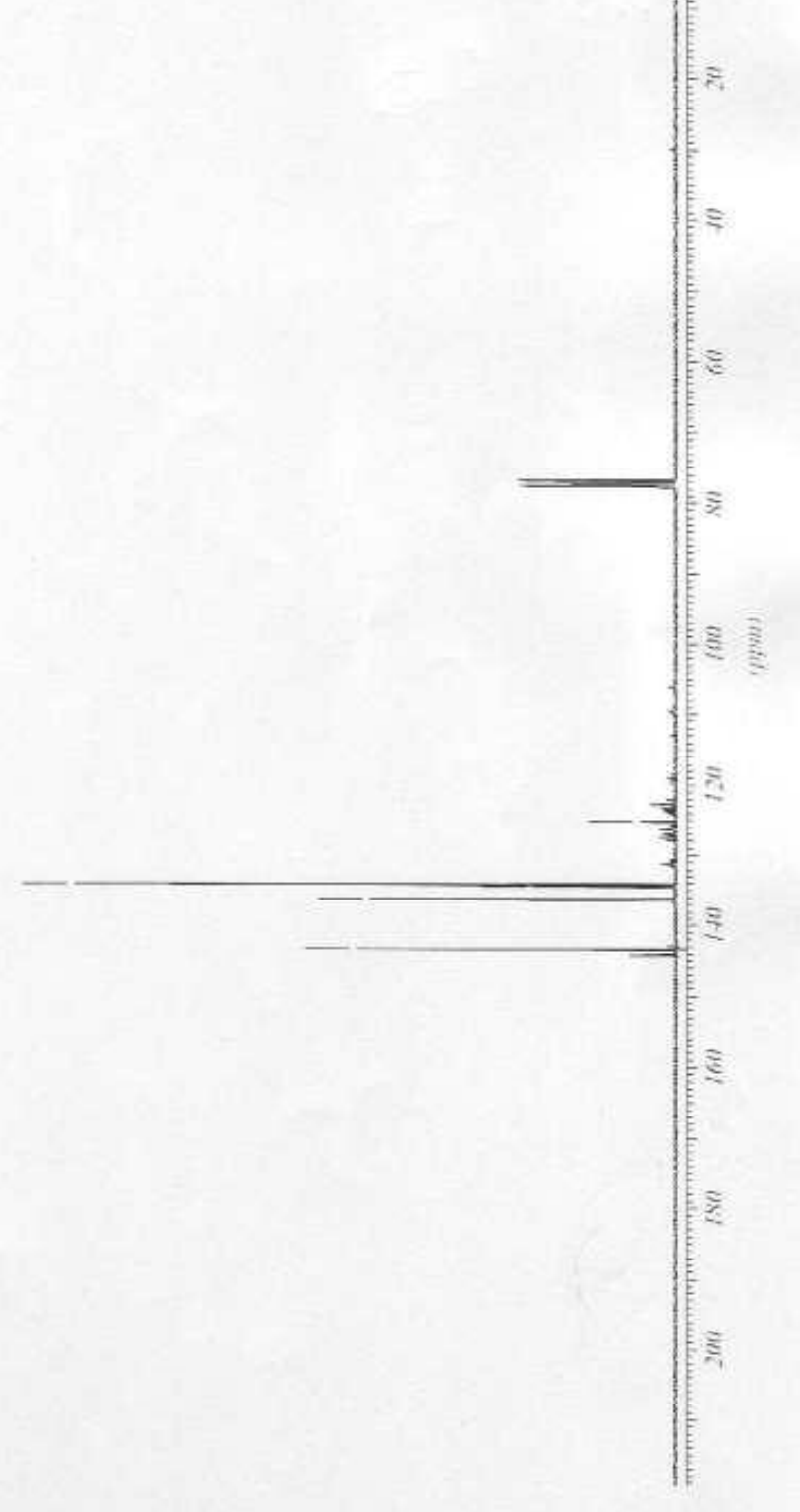



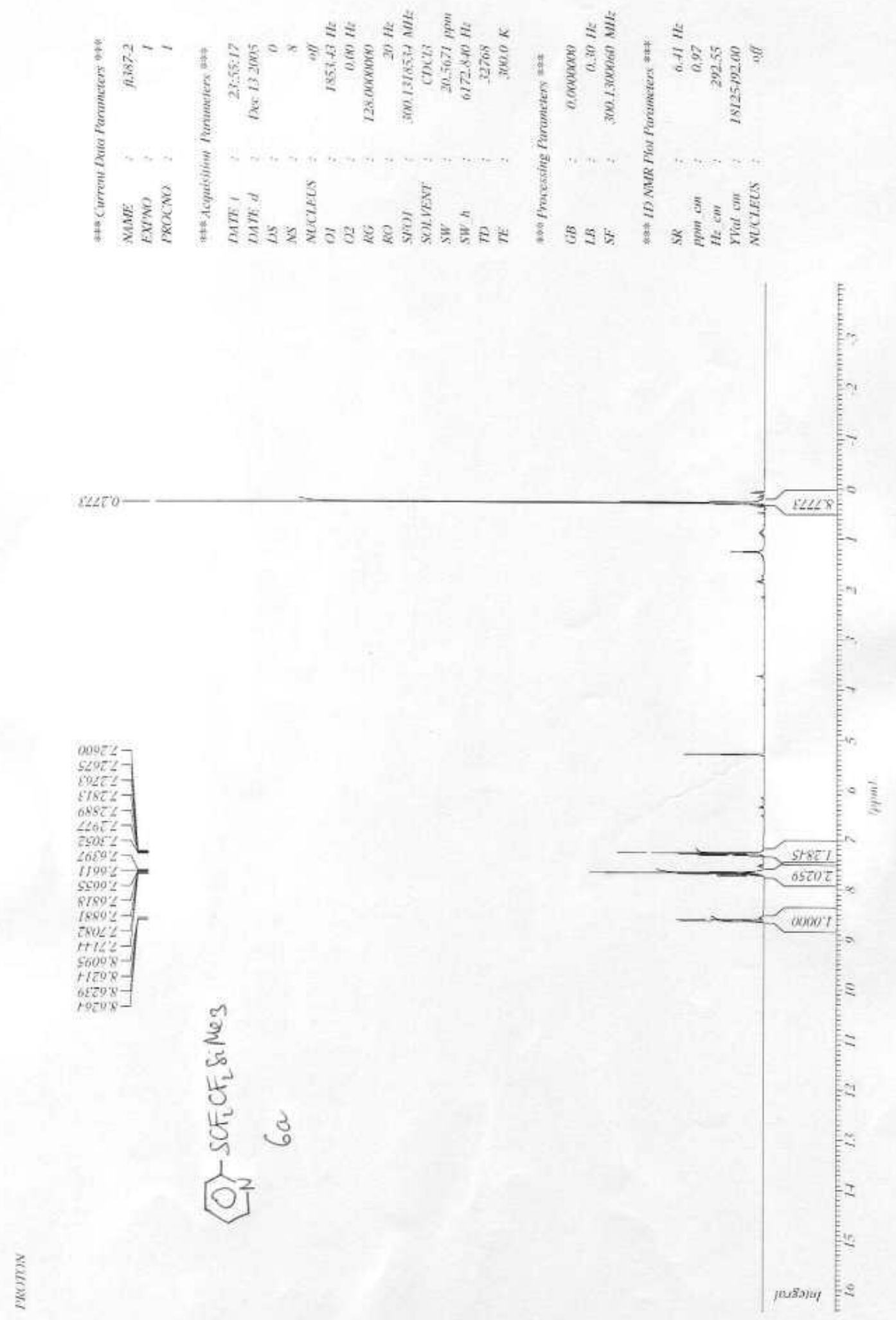


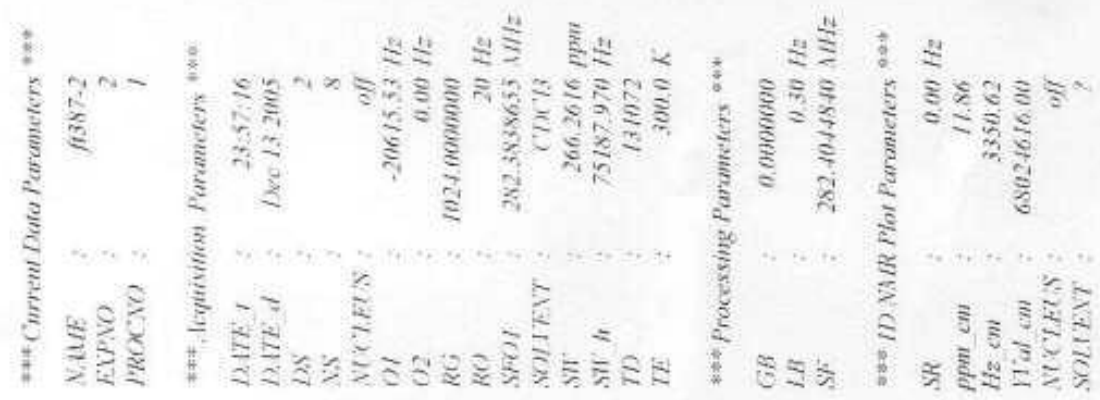

$626+221-7$
$918+221-7$

teyt 221.

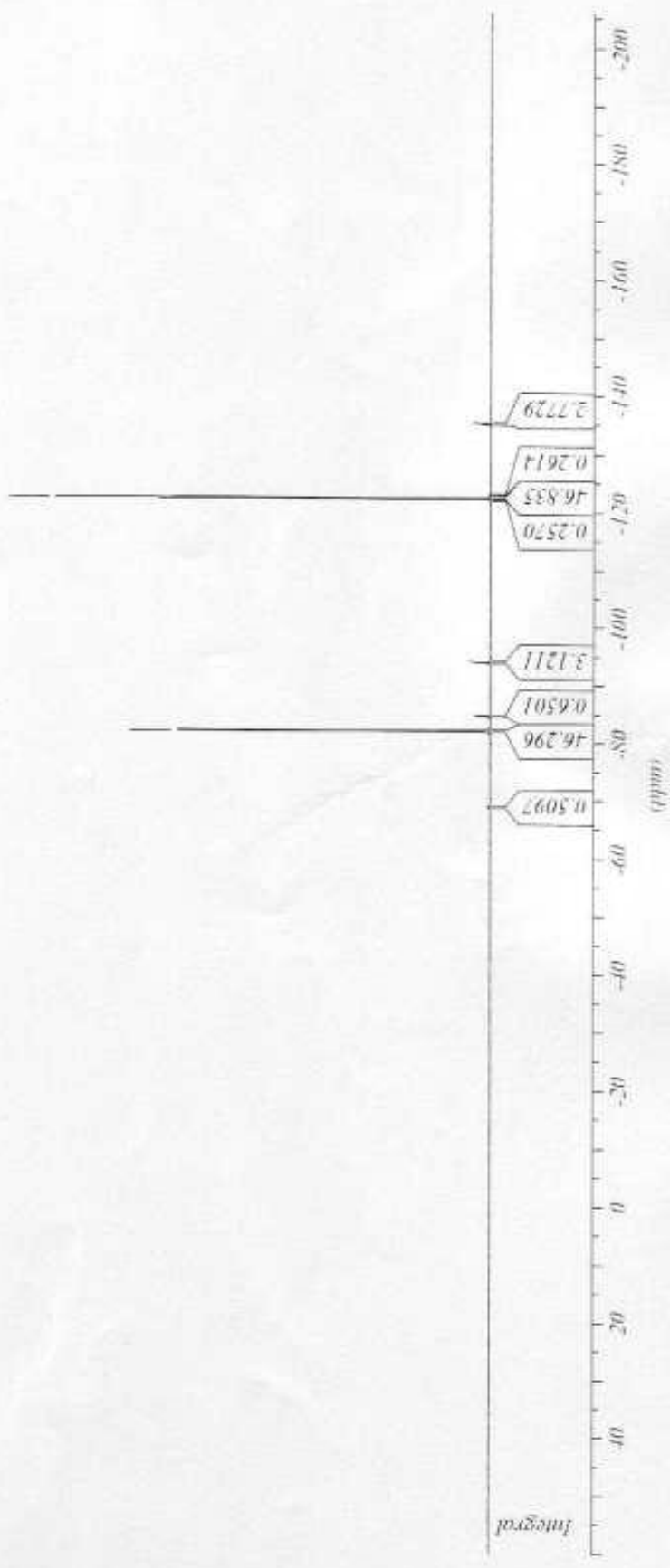




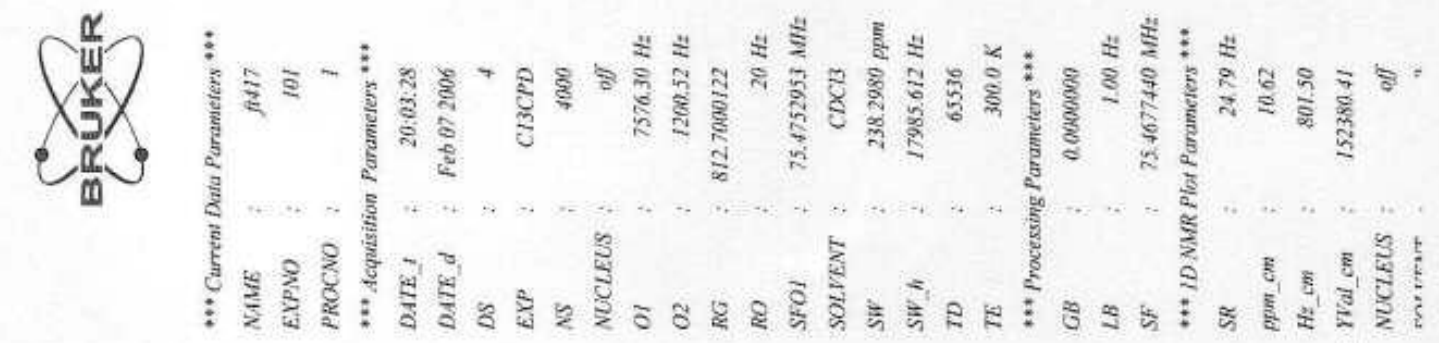
29915 9LET 8511 t $\angle 980^{\circ}$ th $6+90$ '-
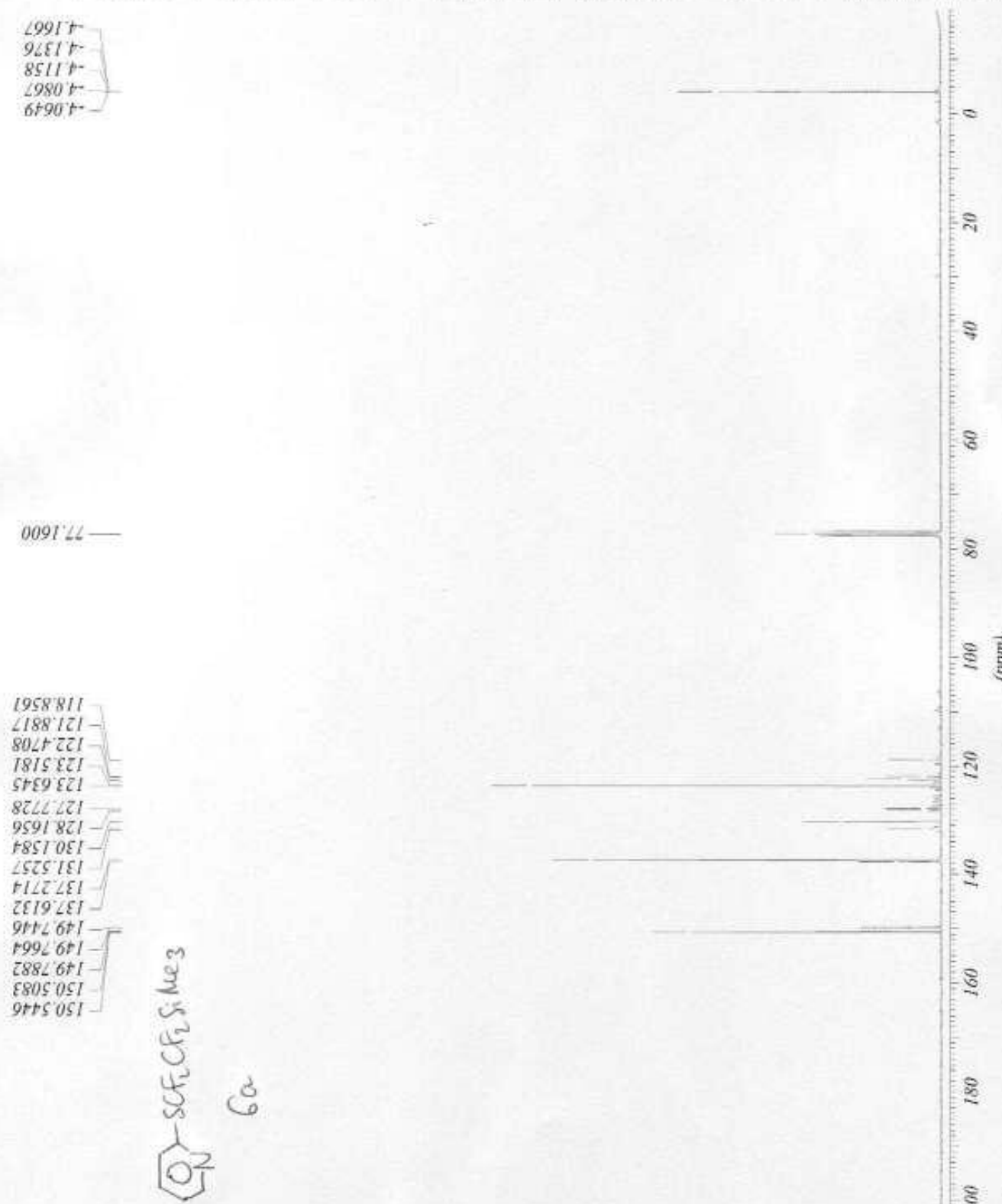

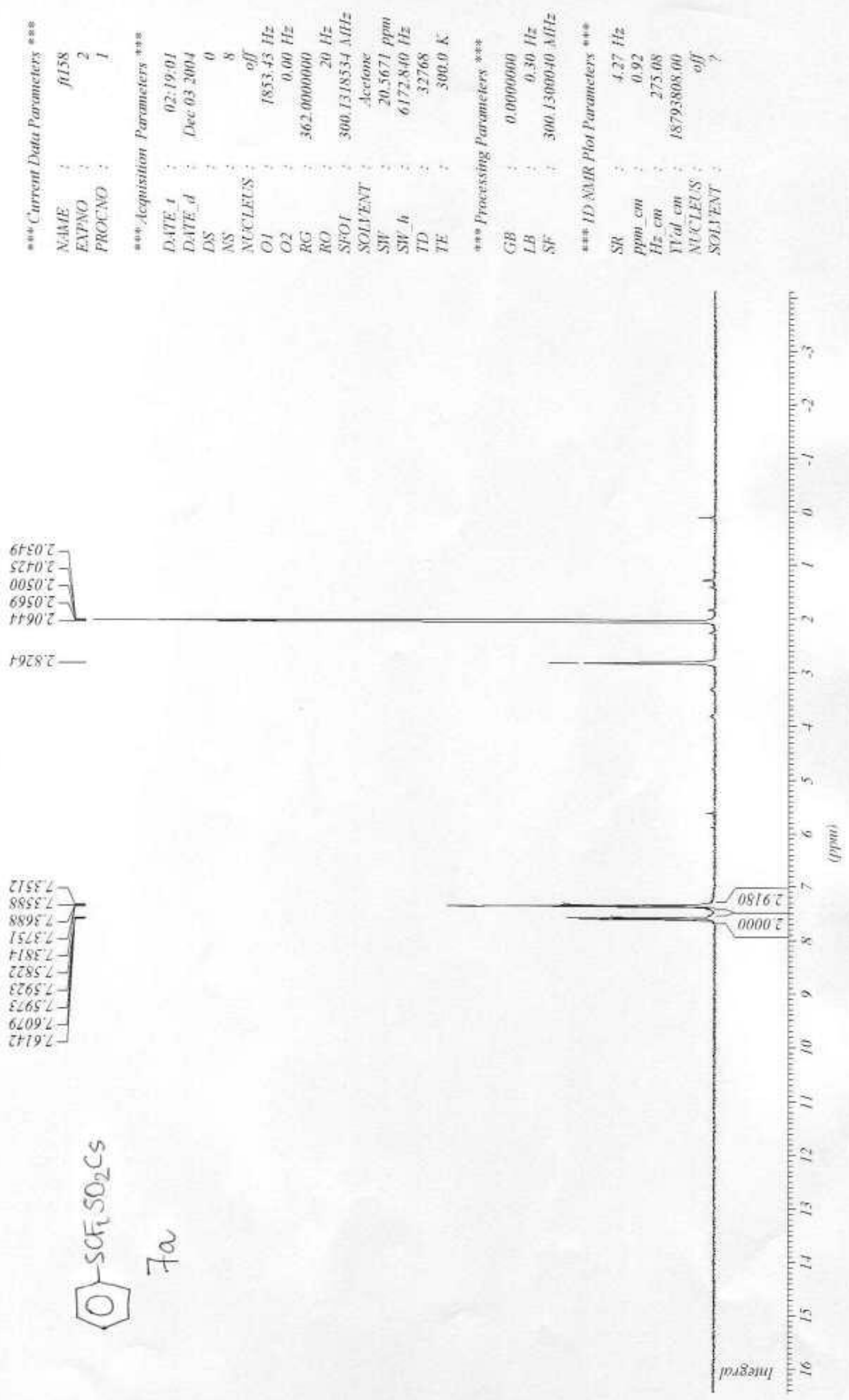

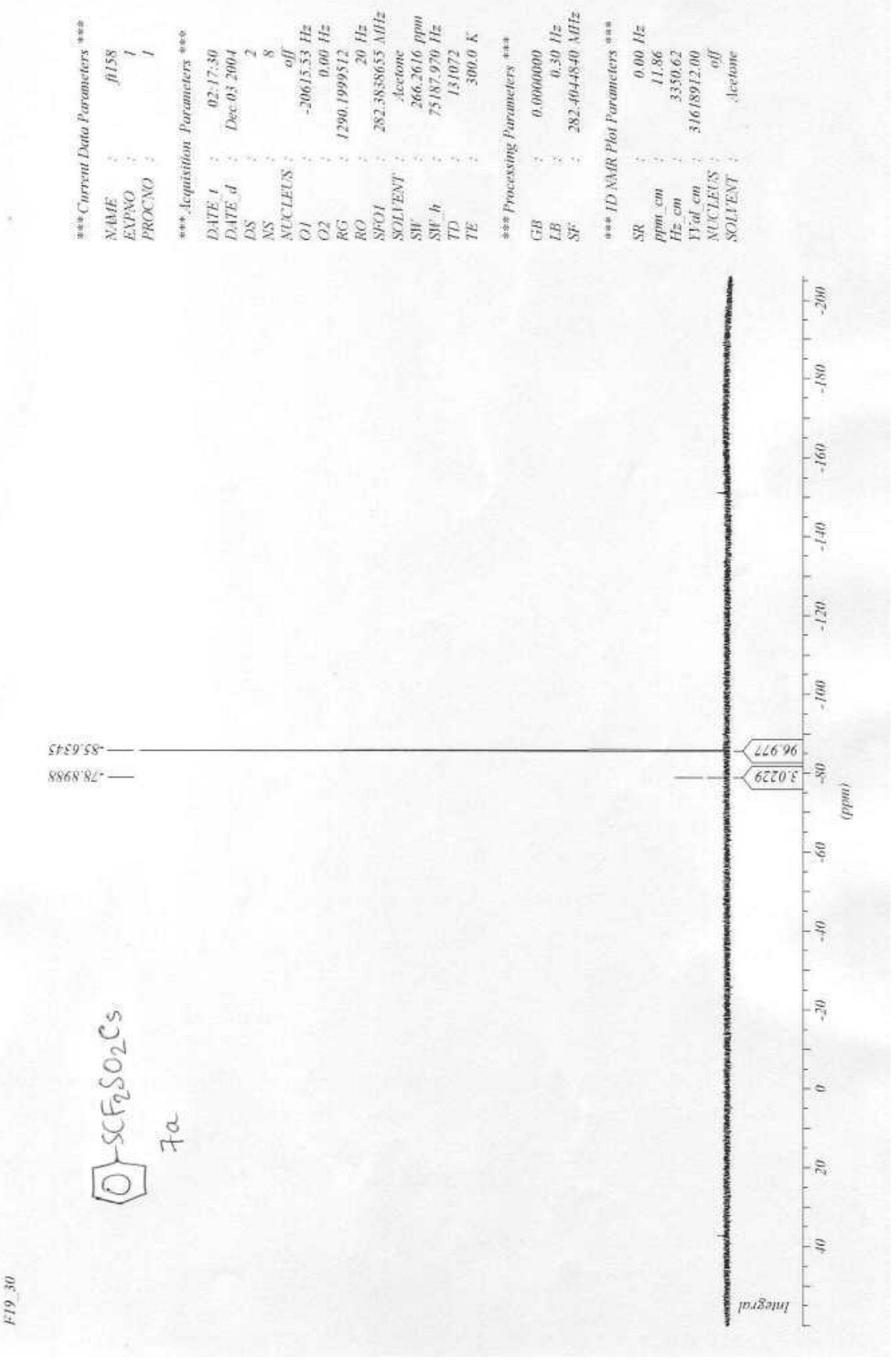

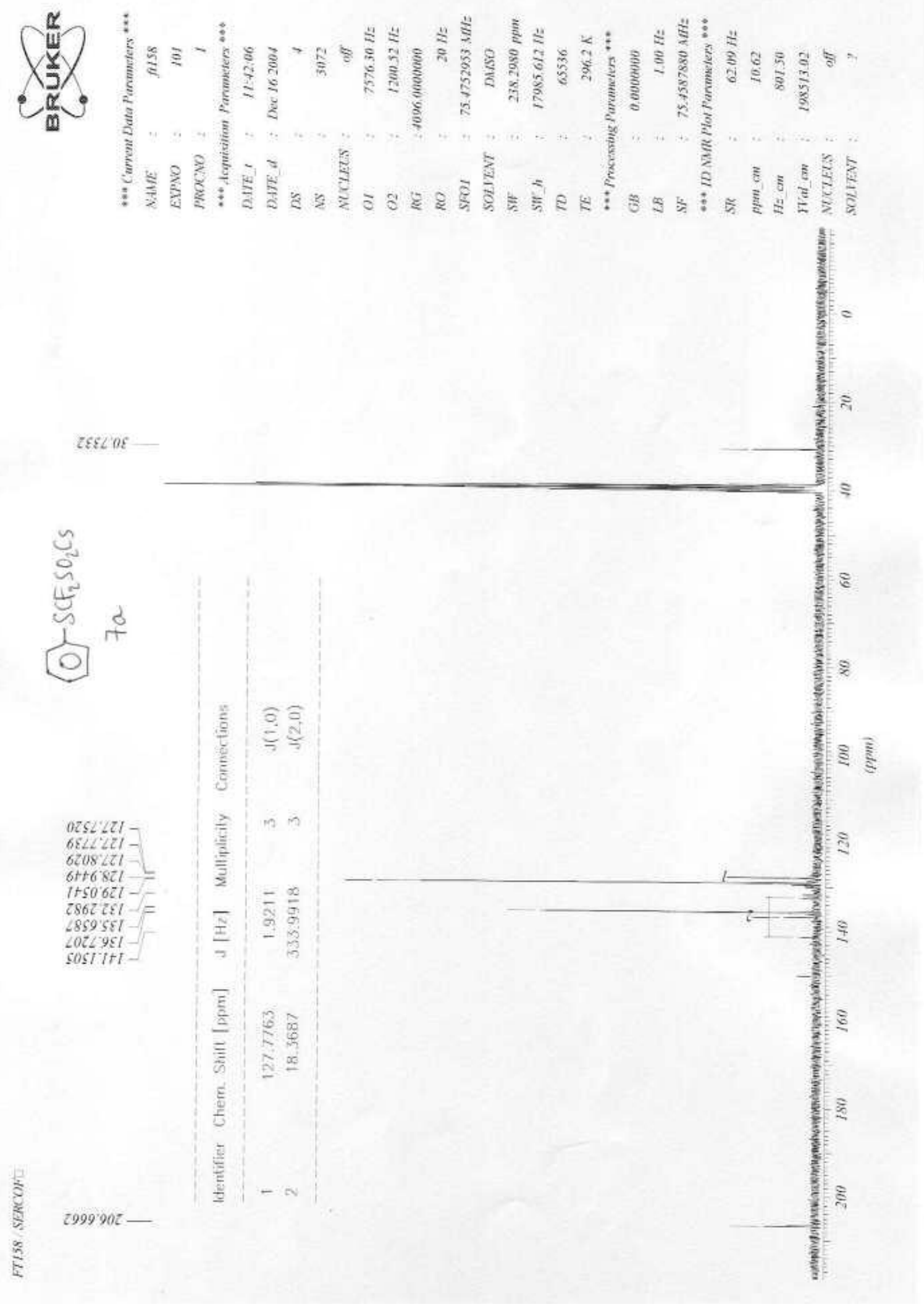


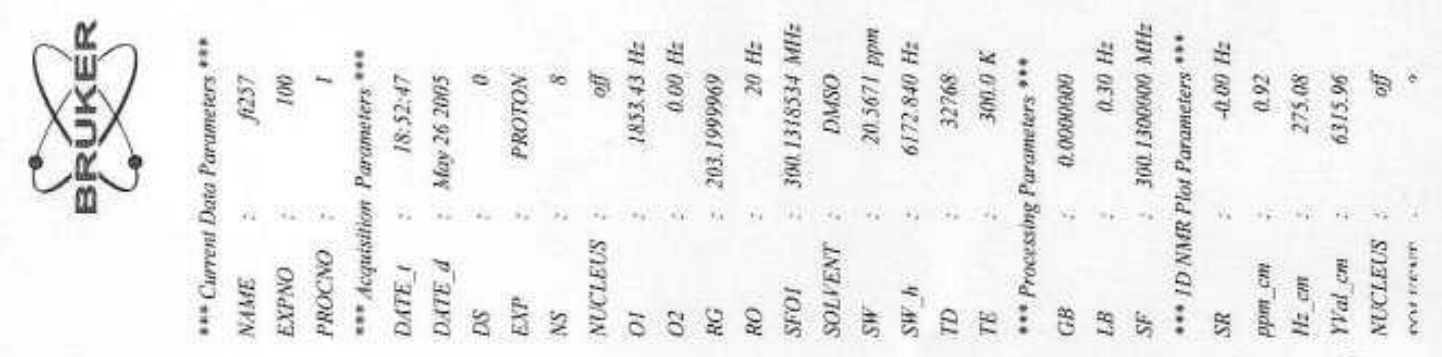

tLos 2

HLEIH -

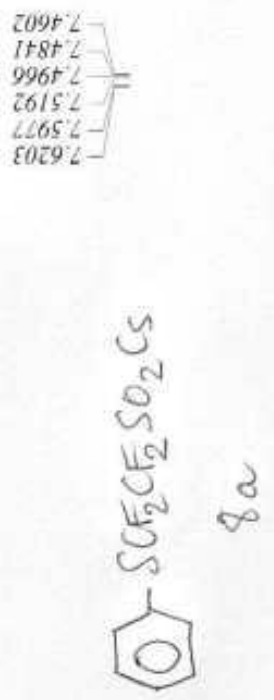



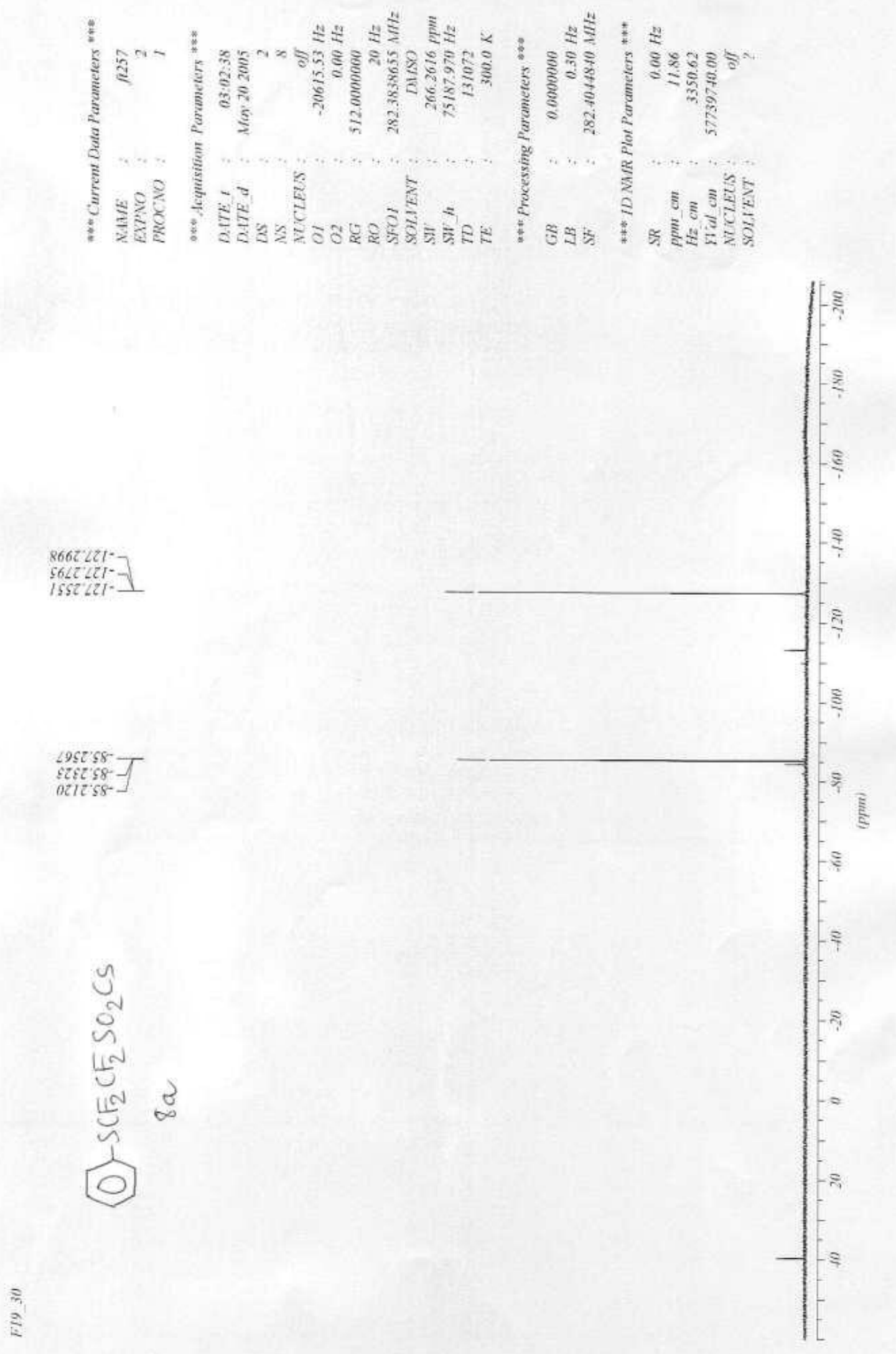

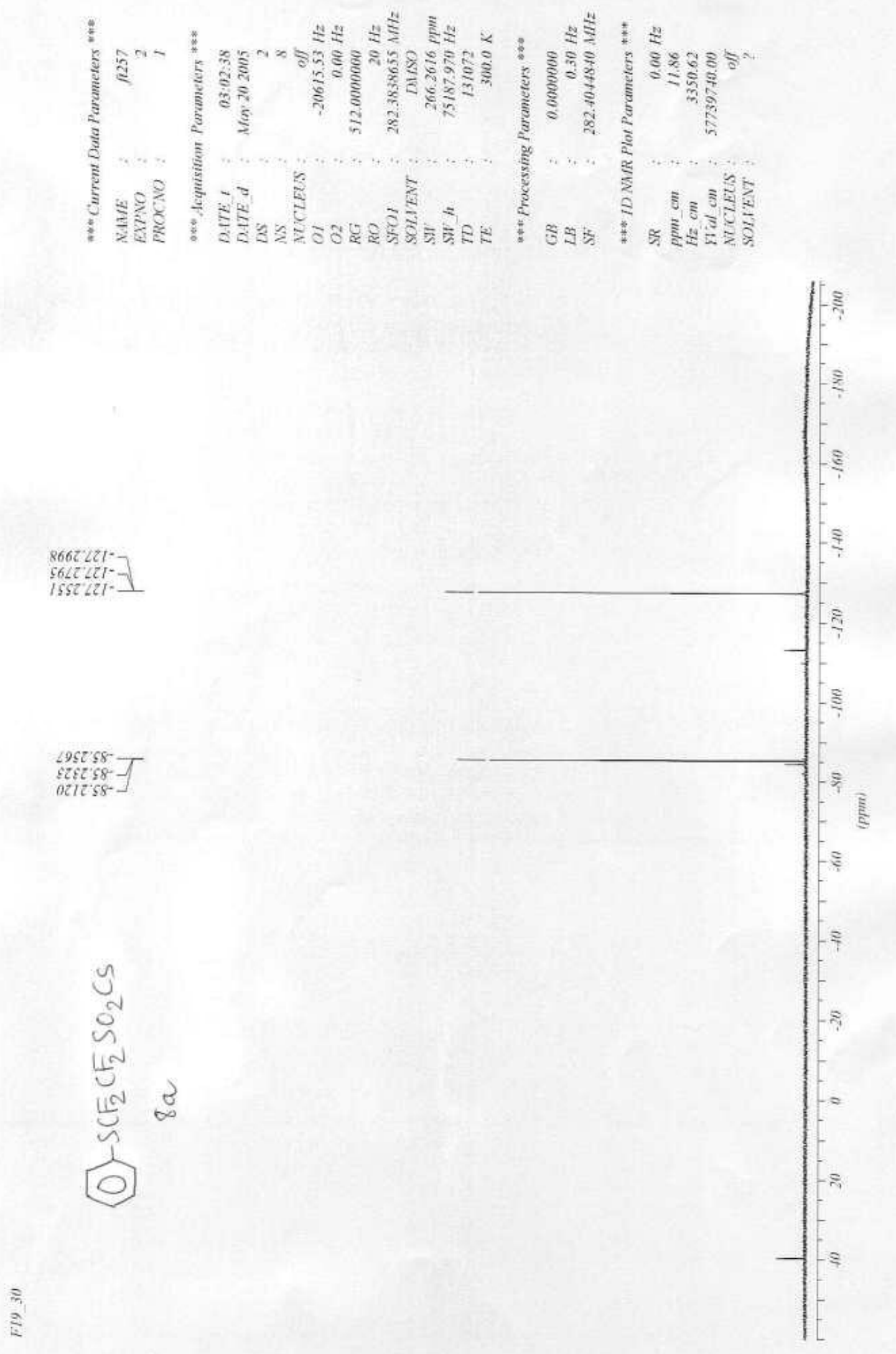


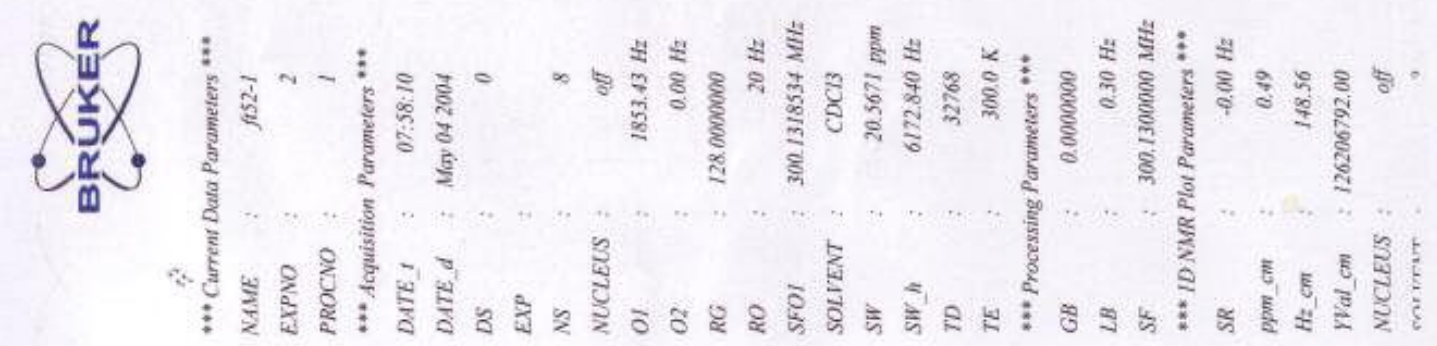

E\&1z०

Heto

$0 / z 6 z$

6226
0586
058

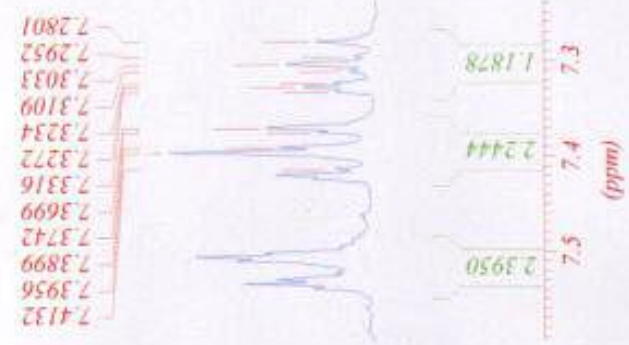

$+5+19$

$\angle 16 E 9$
$606+9$

lose?

¿S62:

EEOE'

60I\&'

TELEL

ZC2E 2

66922

2H 12

6688

())

95682

ZEIT 2

toIt'

cect 2

21052

$+\operatorname{sos} 2$

$860 \mathrm{C}^{\prime} \mathrm{C}$

+225:

zoss

$\angle 8552$

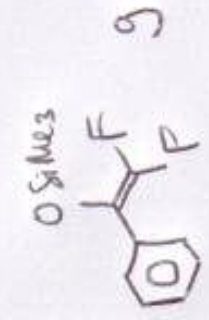

\&

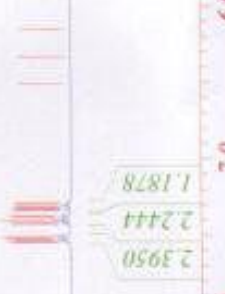

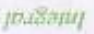



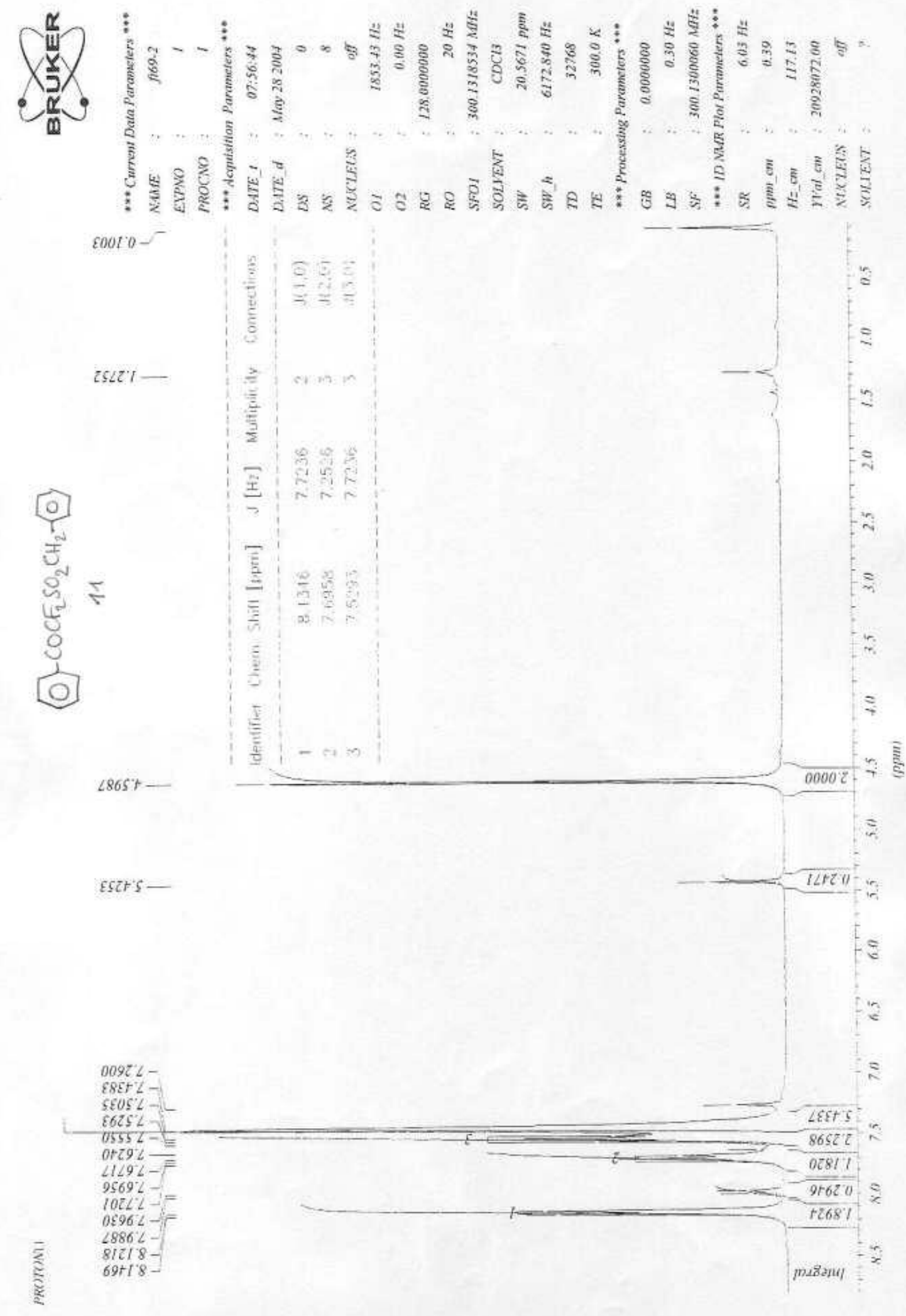

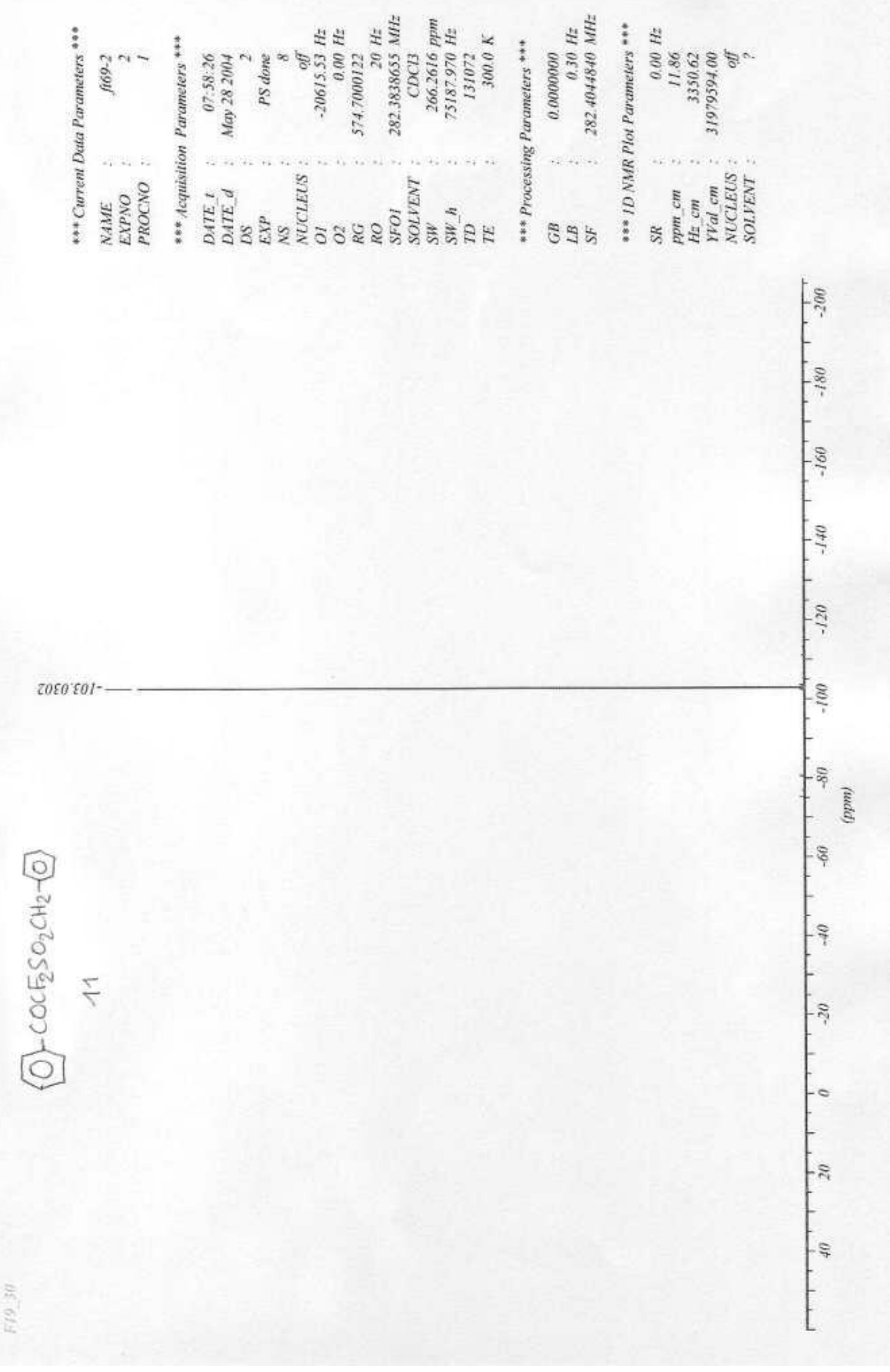


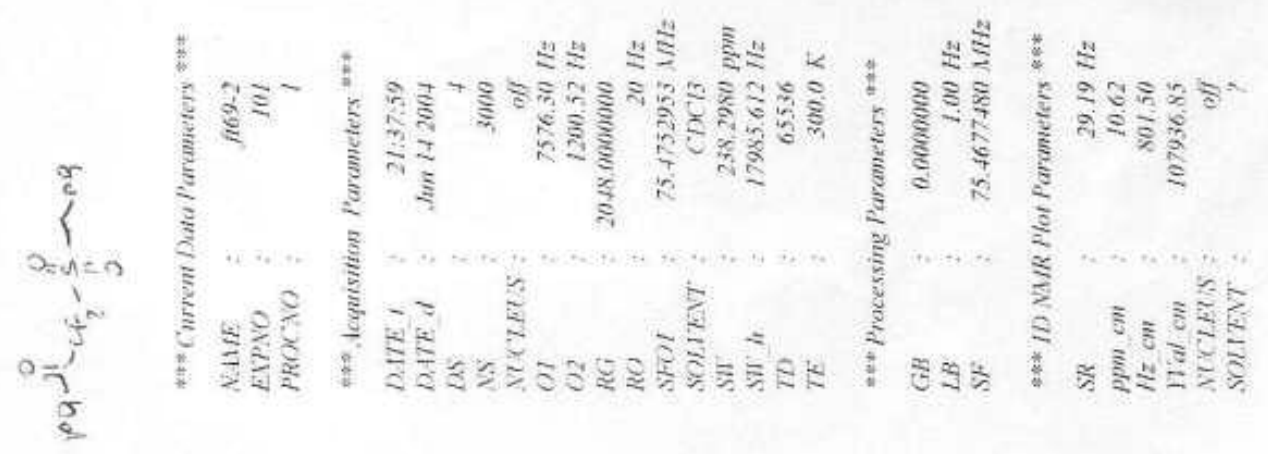

$\angle O E T-$

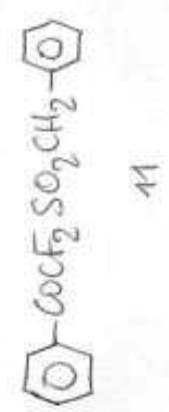

scot 95

$098529-$

esse $92-$

$1609122=$

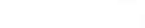

256211
2596911

$9596011-$
$962001]$

$I 8528 Z I$

$2689821-$

20128 $321-$

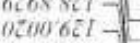

бeEl $6: 1-$

$+8526 z I$

HLWDE-

STE DE -

IIGLOEI -

CSST $75 T-$

IEZ TEI

$99627 E 7$

F8S TEI.

at-o cal

6tz $25 \mathrm{sg}]$

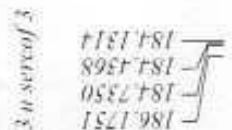

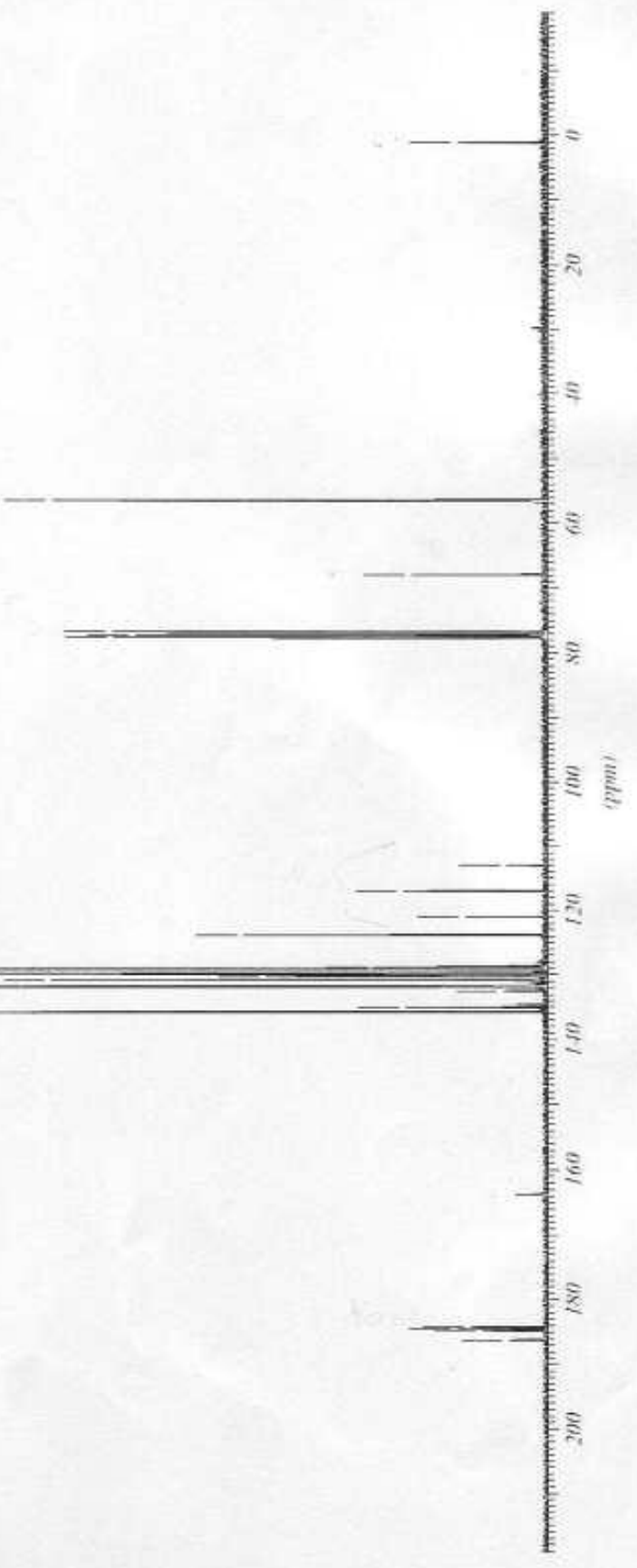



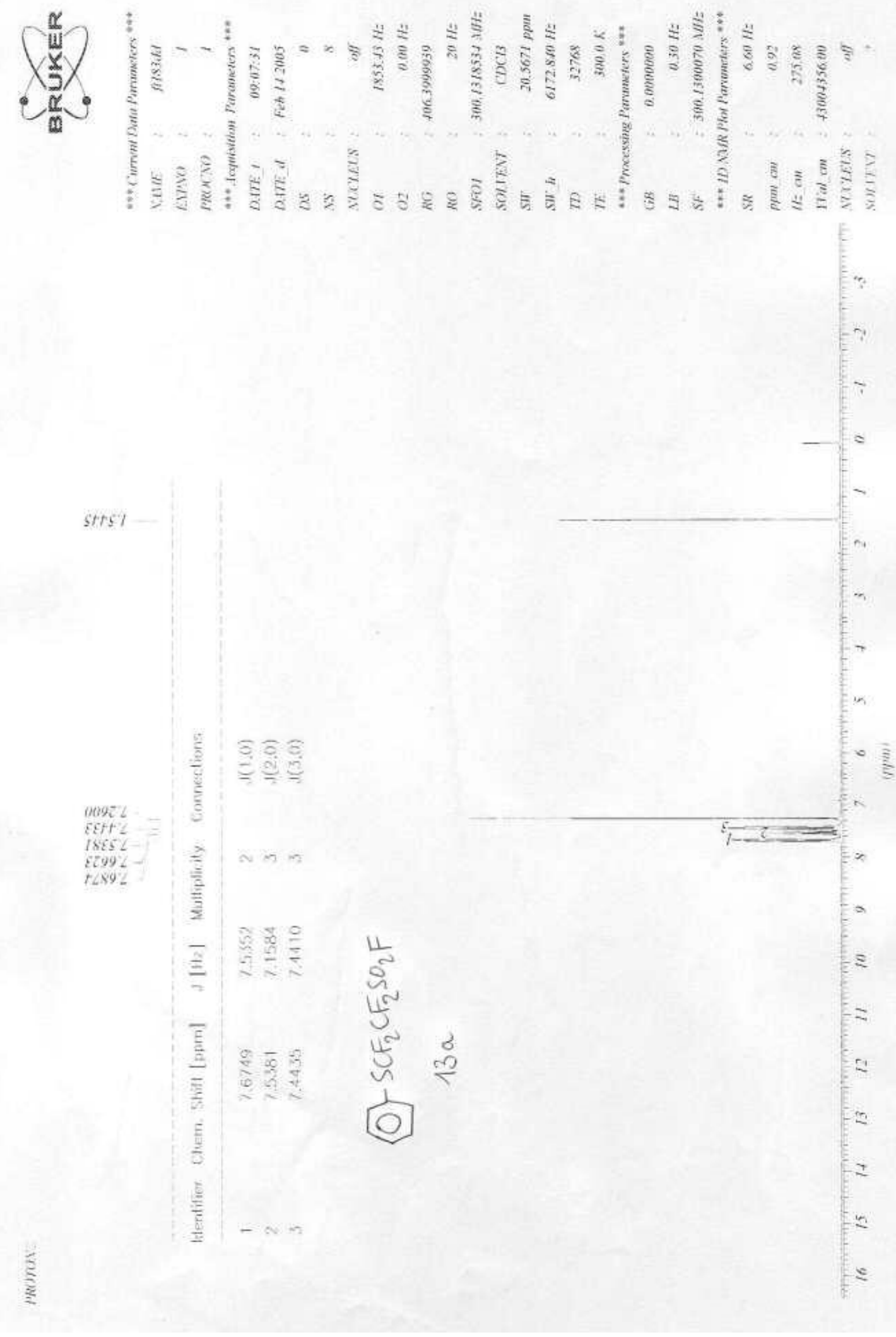

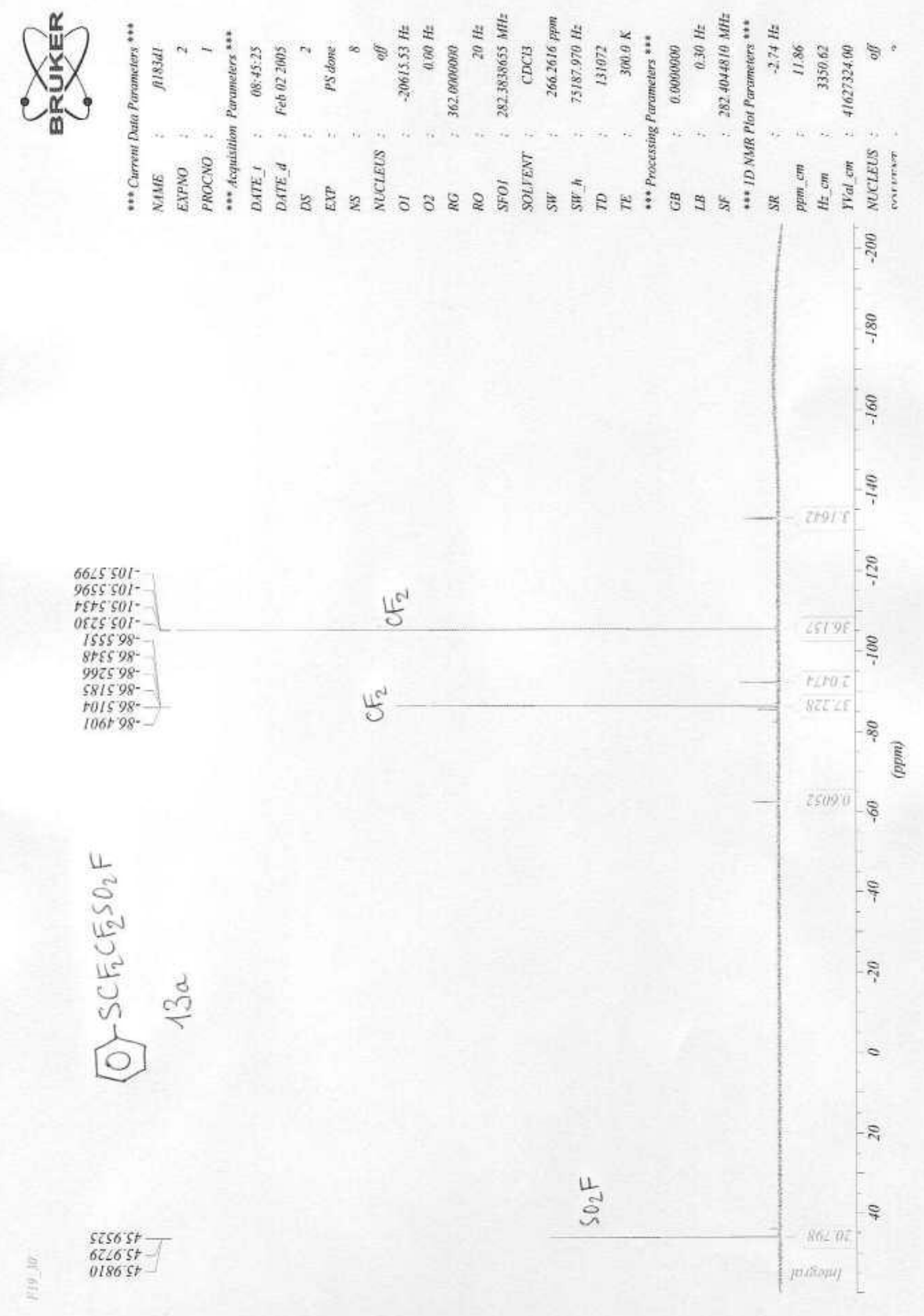


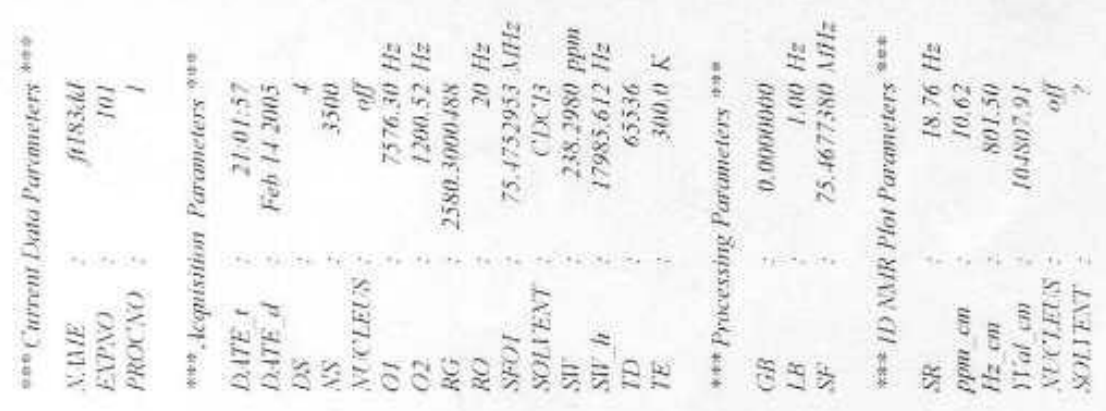

109912L

$2 s+12]$

+269671
cI 6171$]$

ti06 211

$185-721$
$9660221]$

SEl9 द्वा

ocos $621-5$

SYL97E7

i2s 25

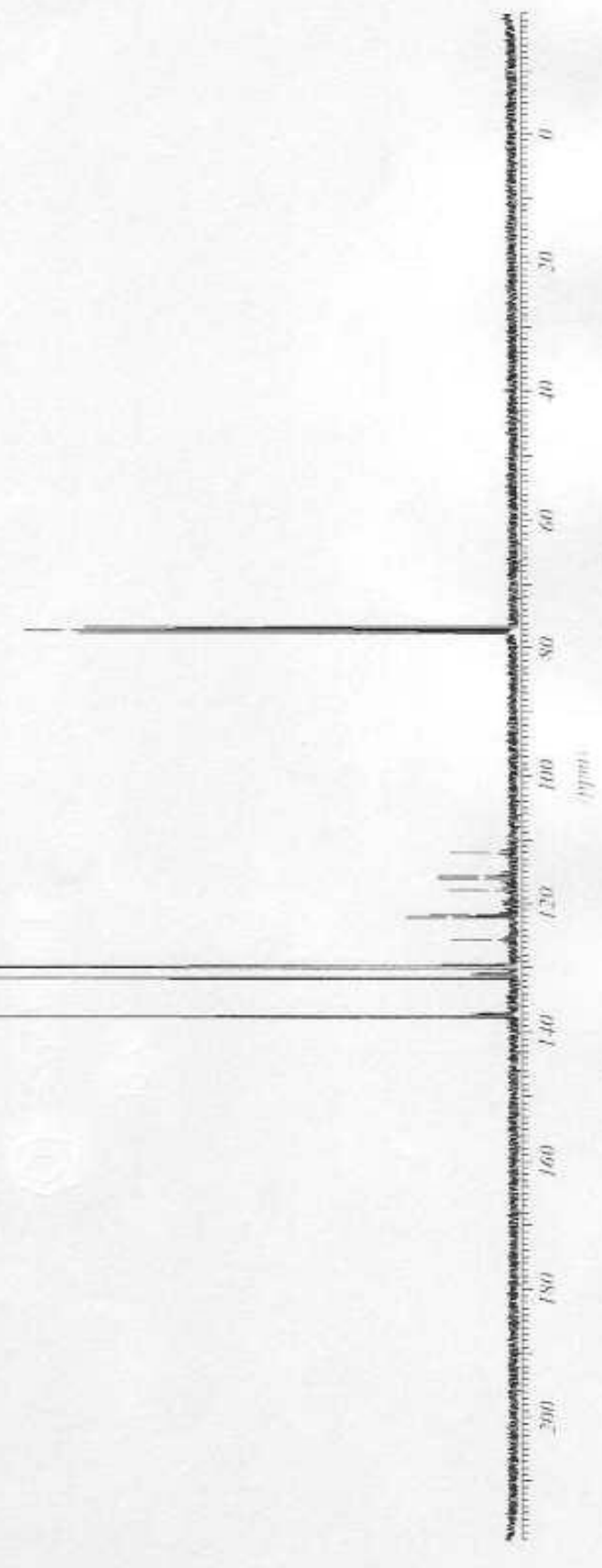




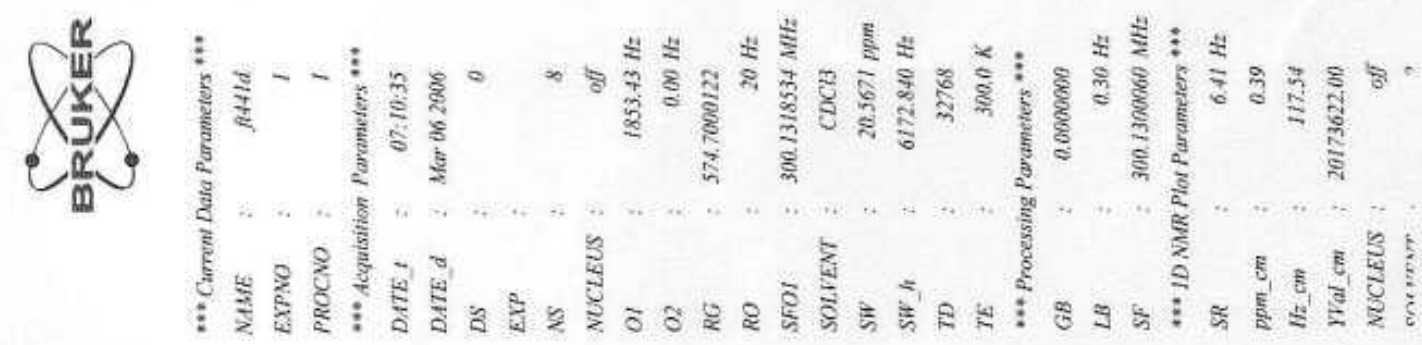

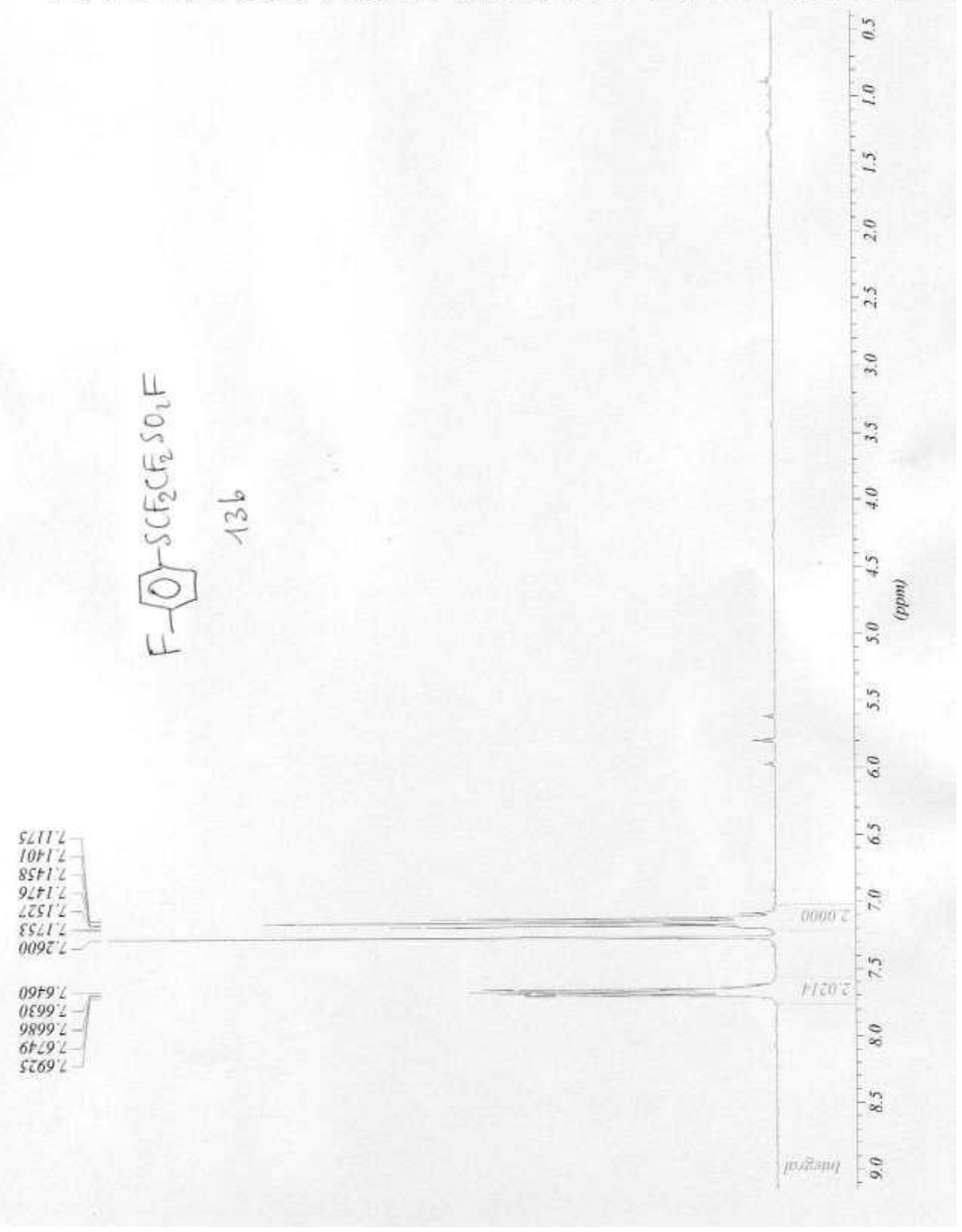




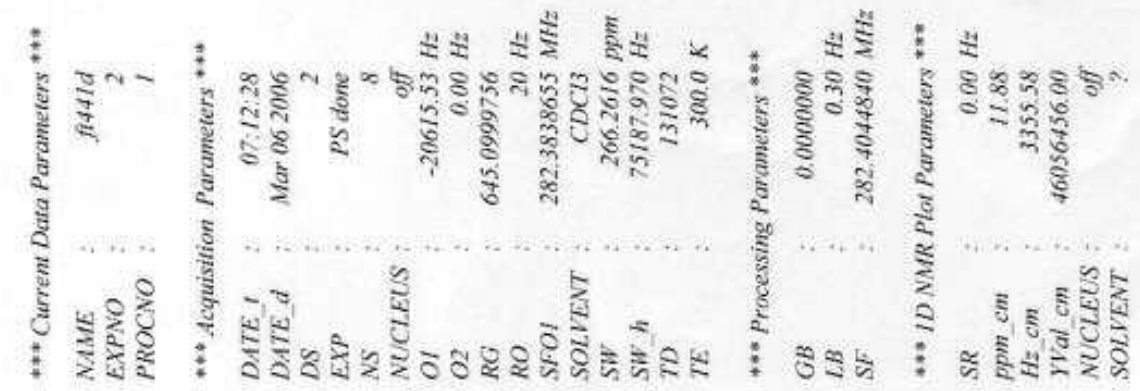

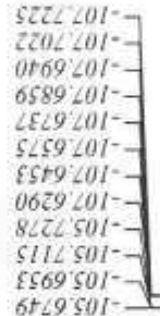

$6 \angle 20 \div 8$

$9<00<8^{-}-1$

$566698^{-}-$

$+16698^{-1}-$

$288698^{-1}-$

679698 -

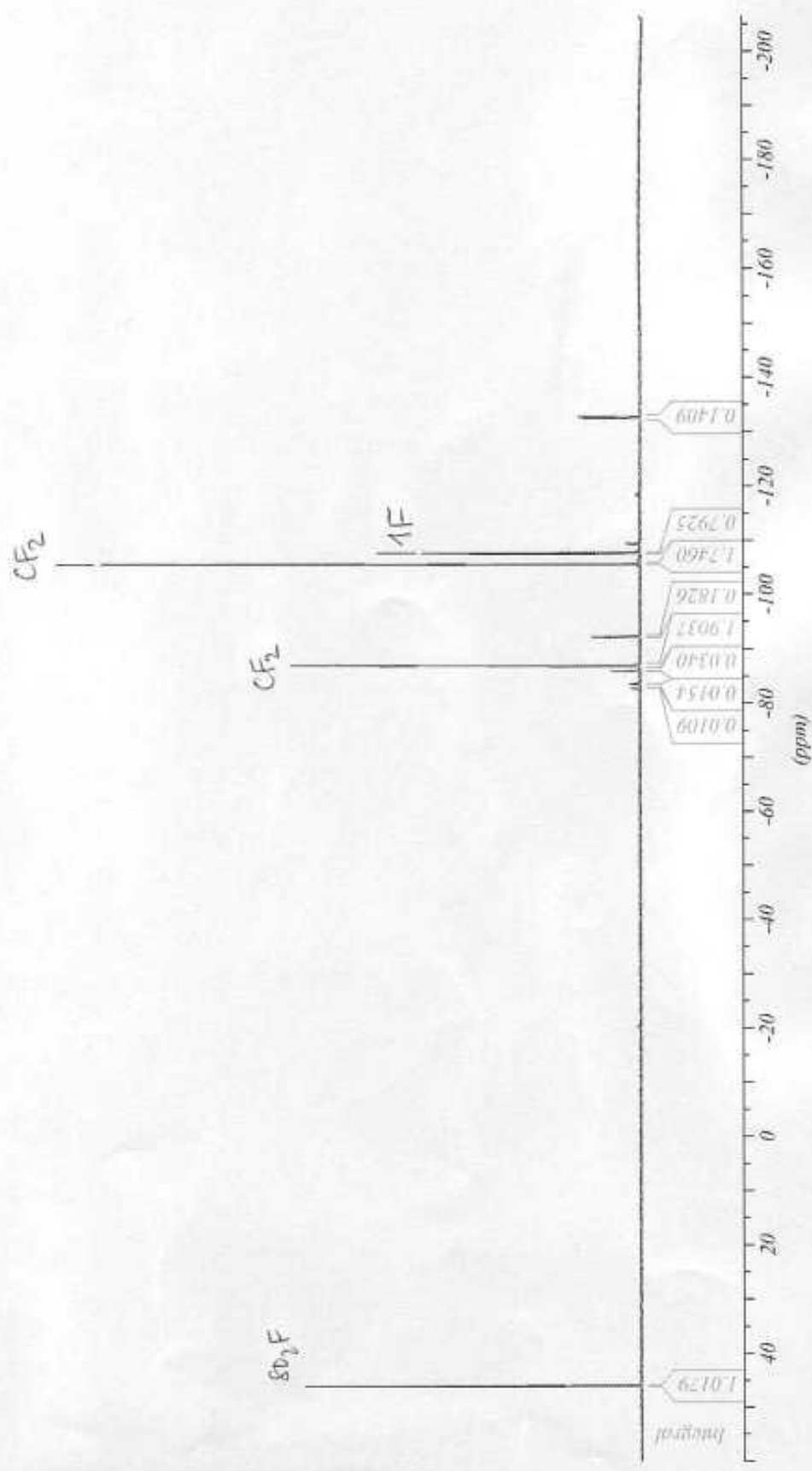



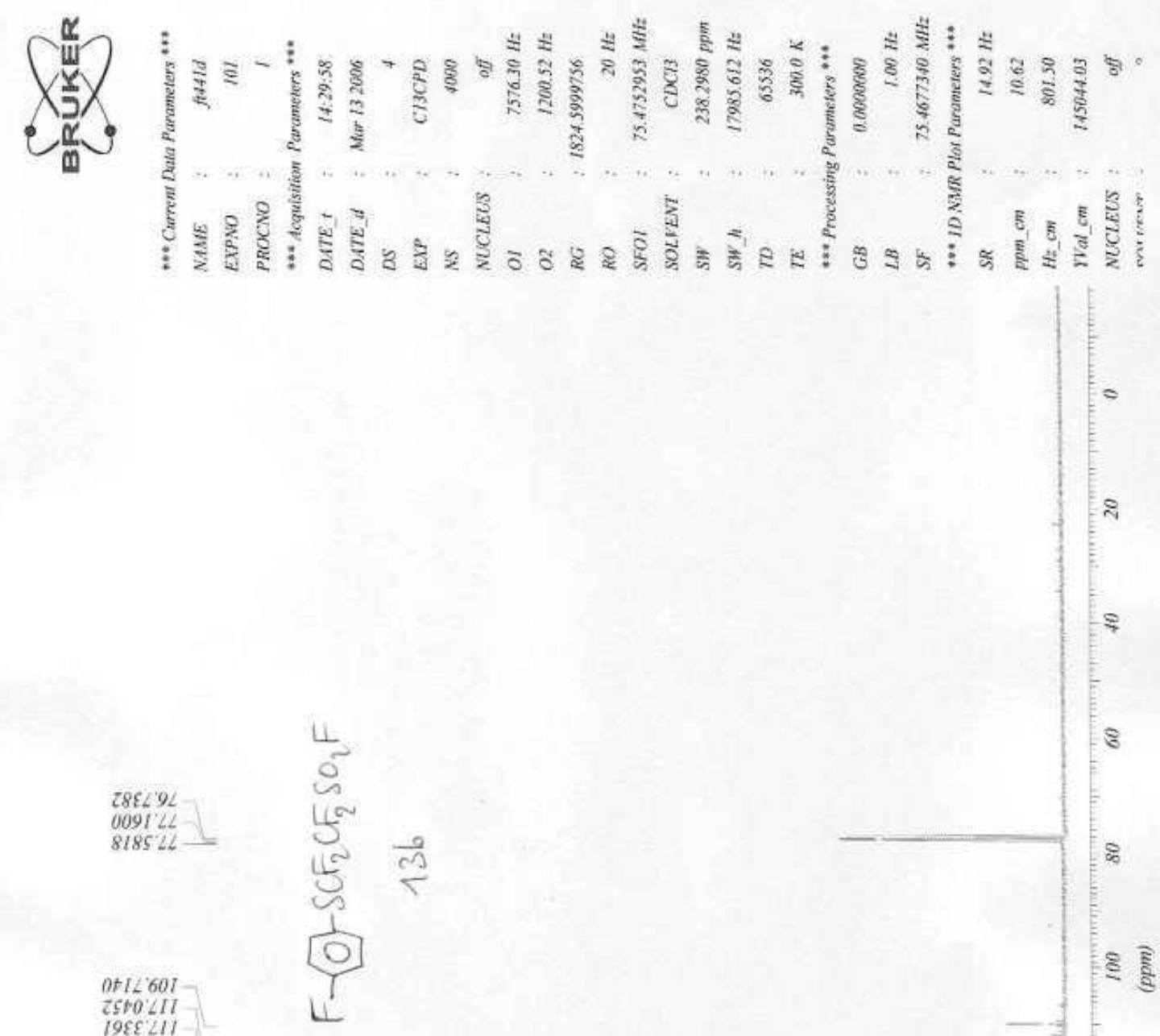

$889+891$ $6828991-$

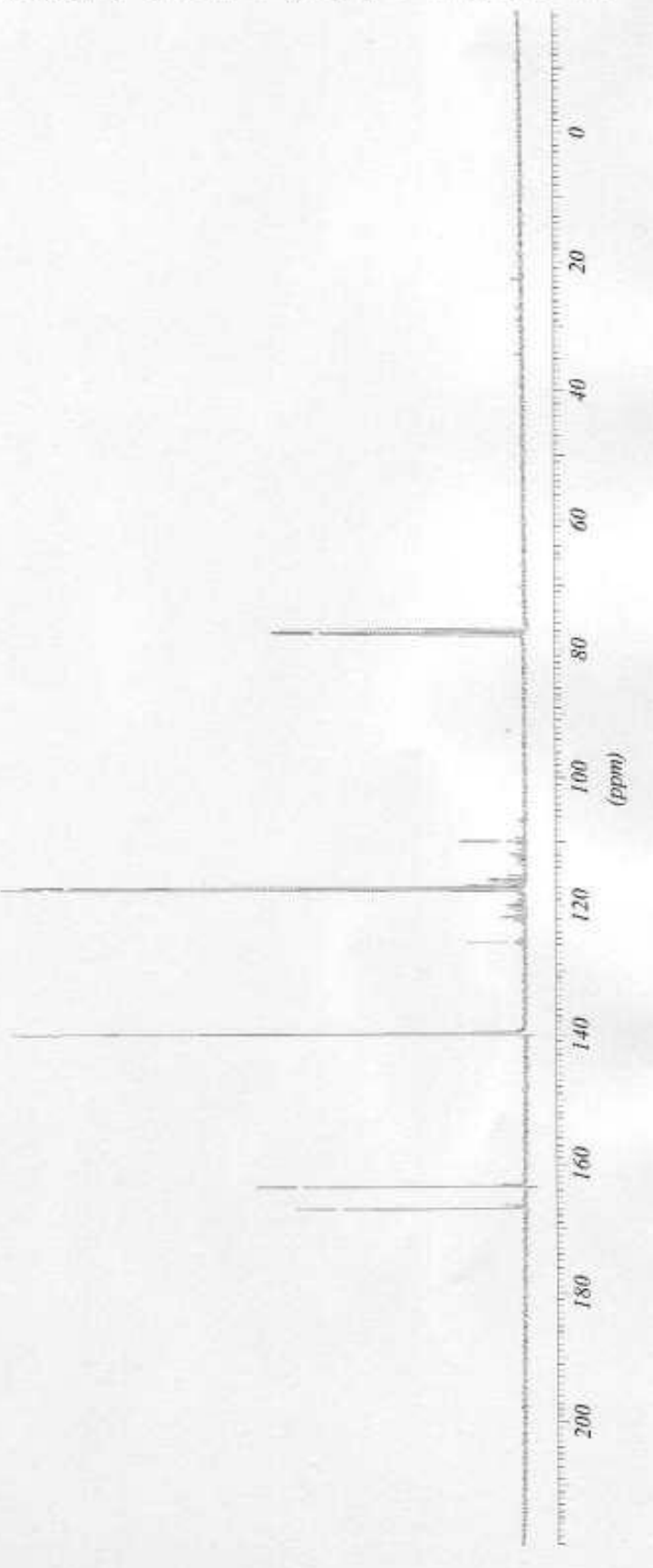




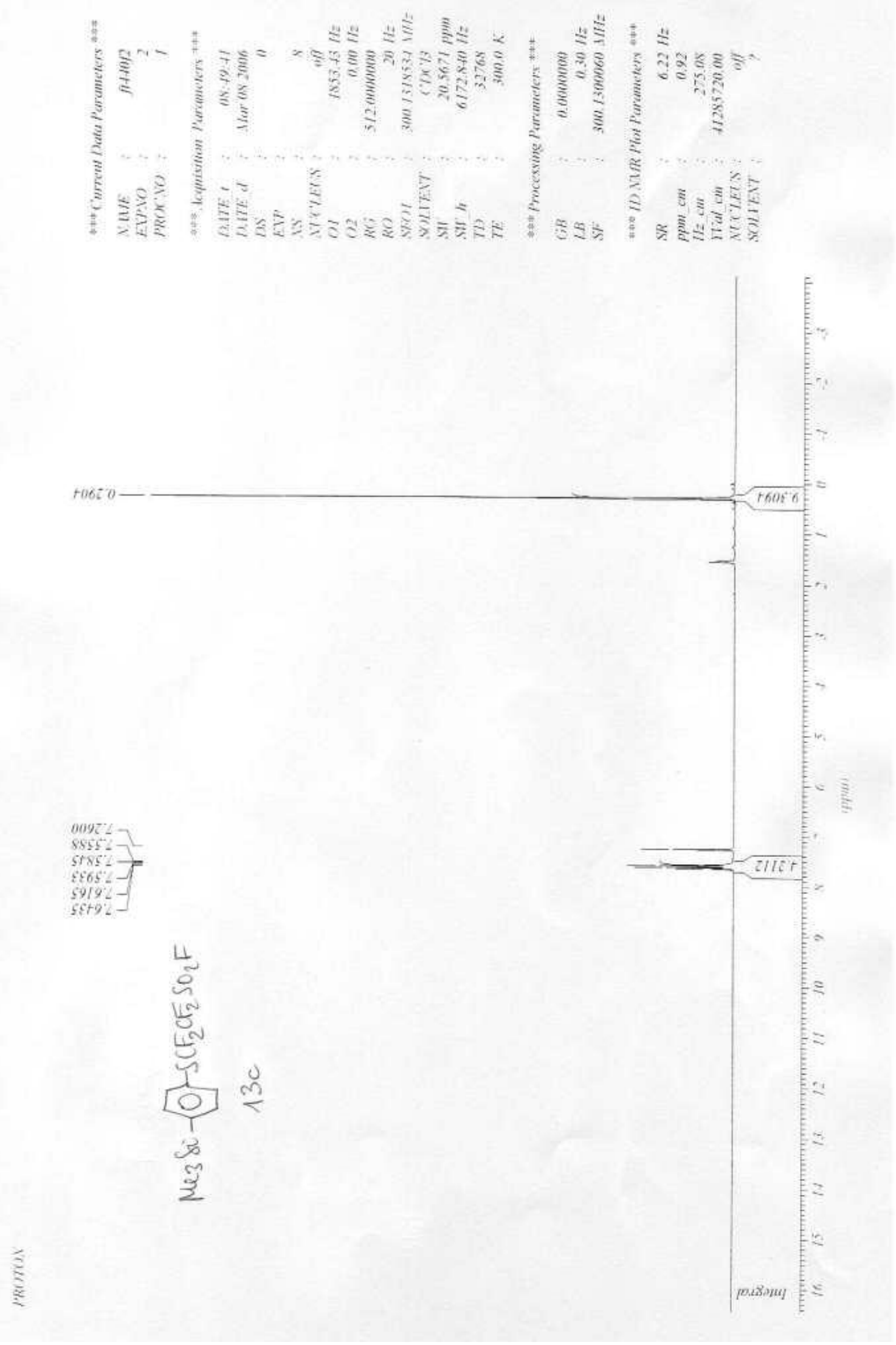



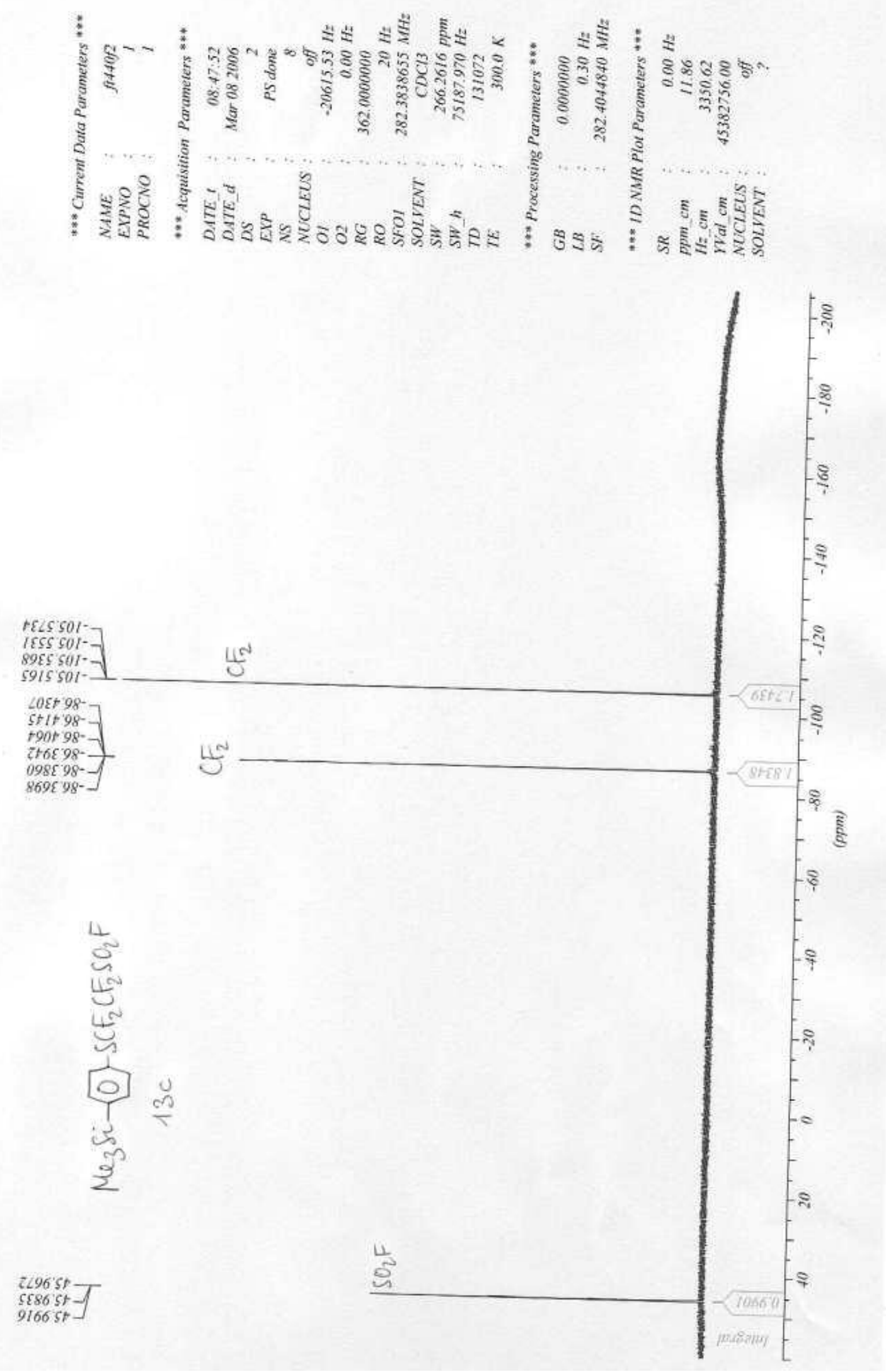

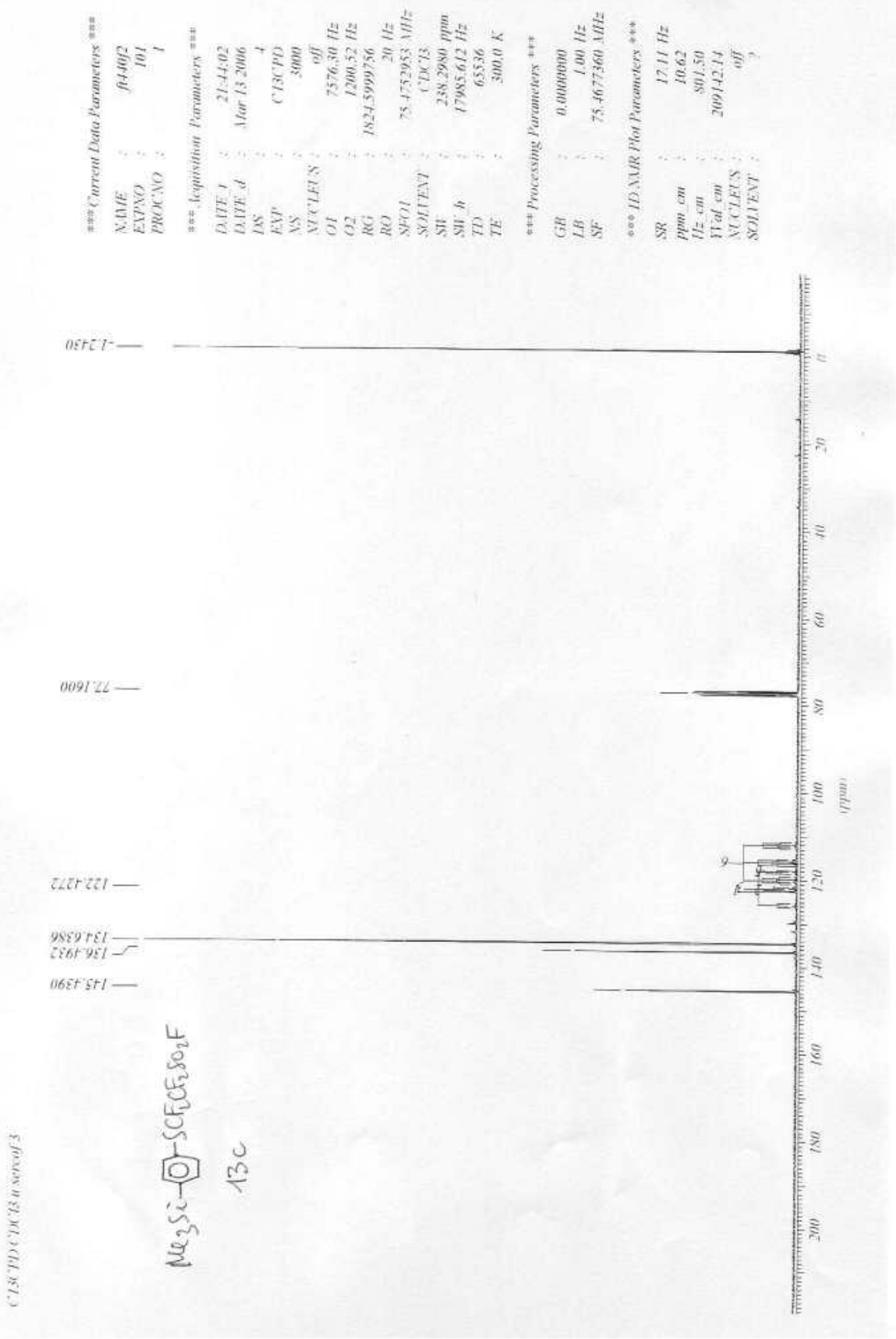

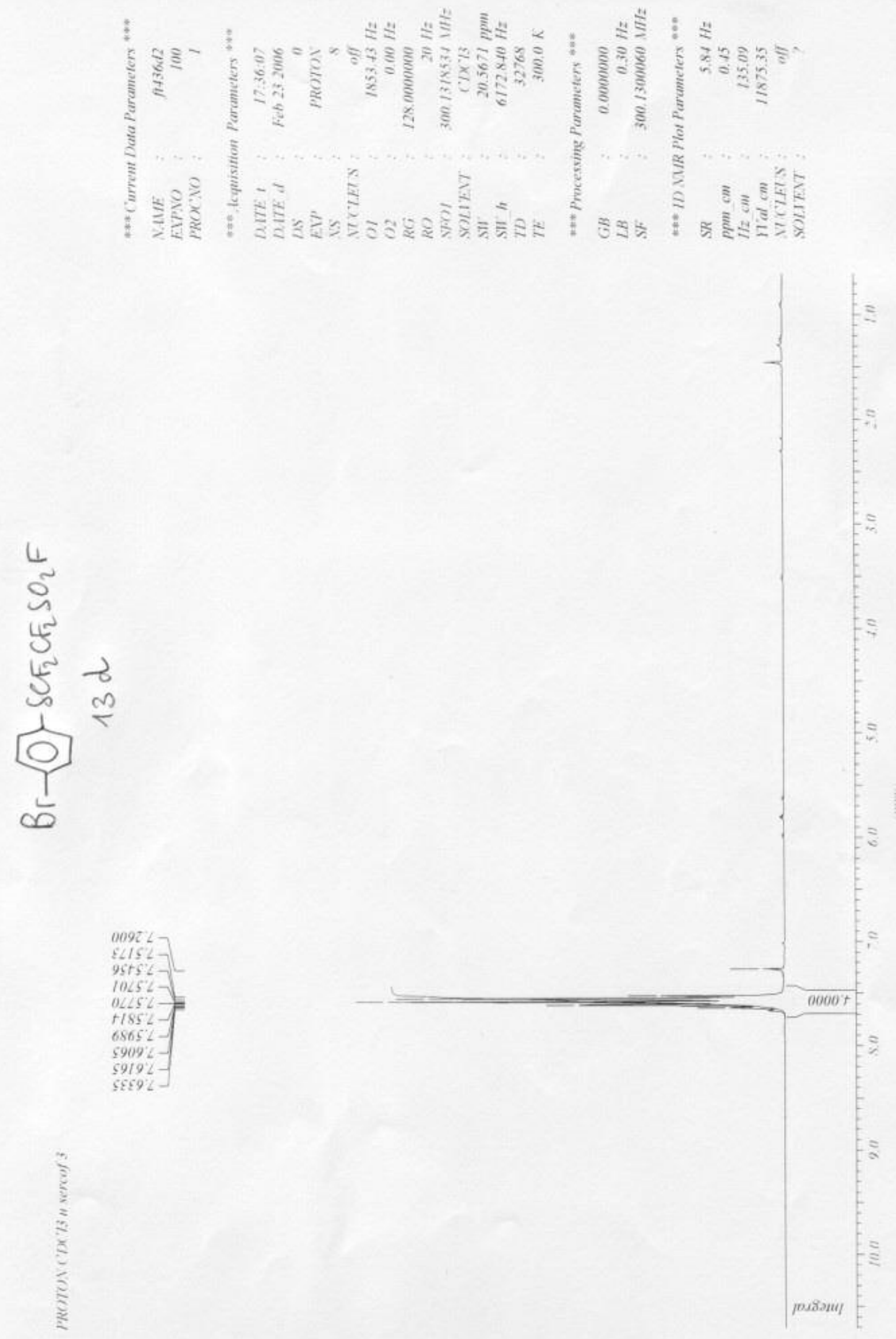

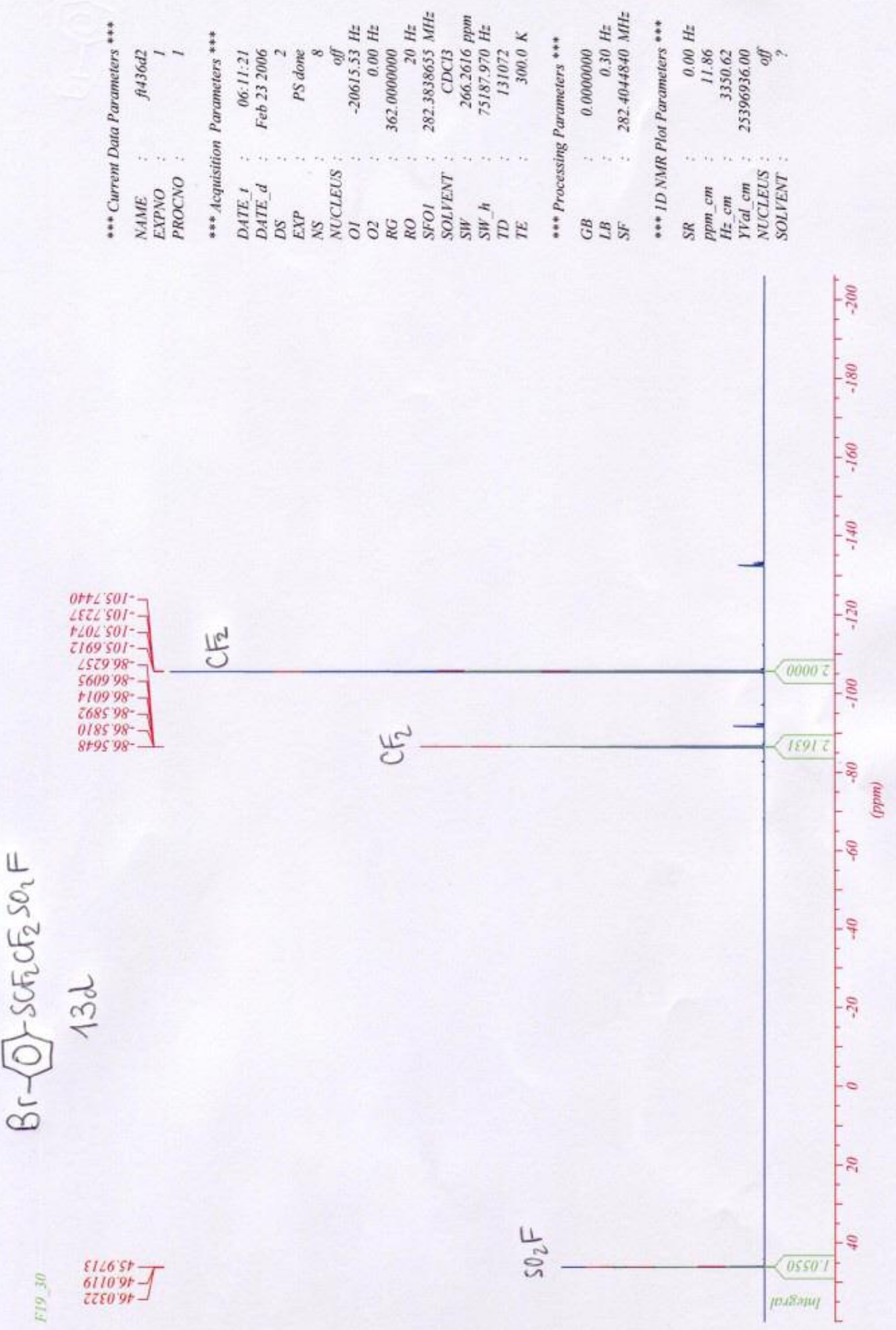

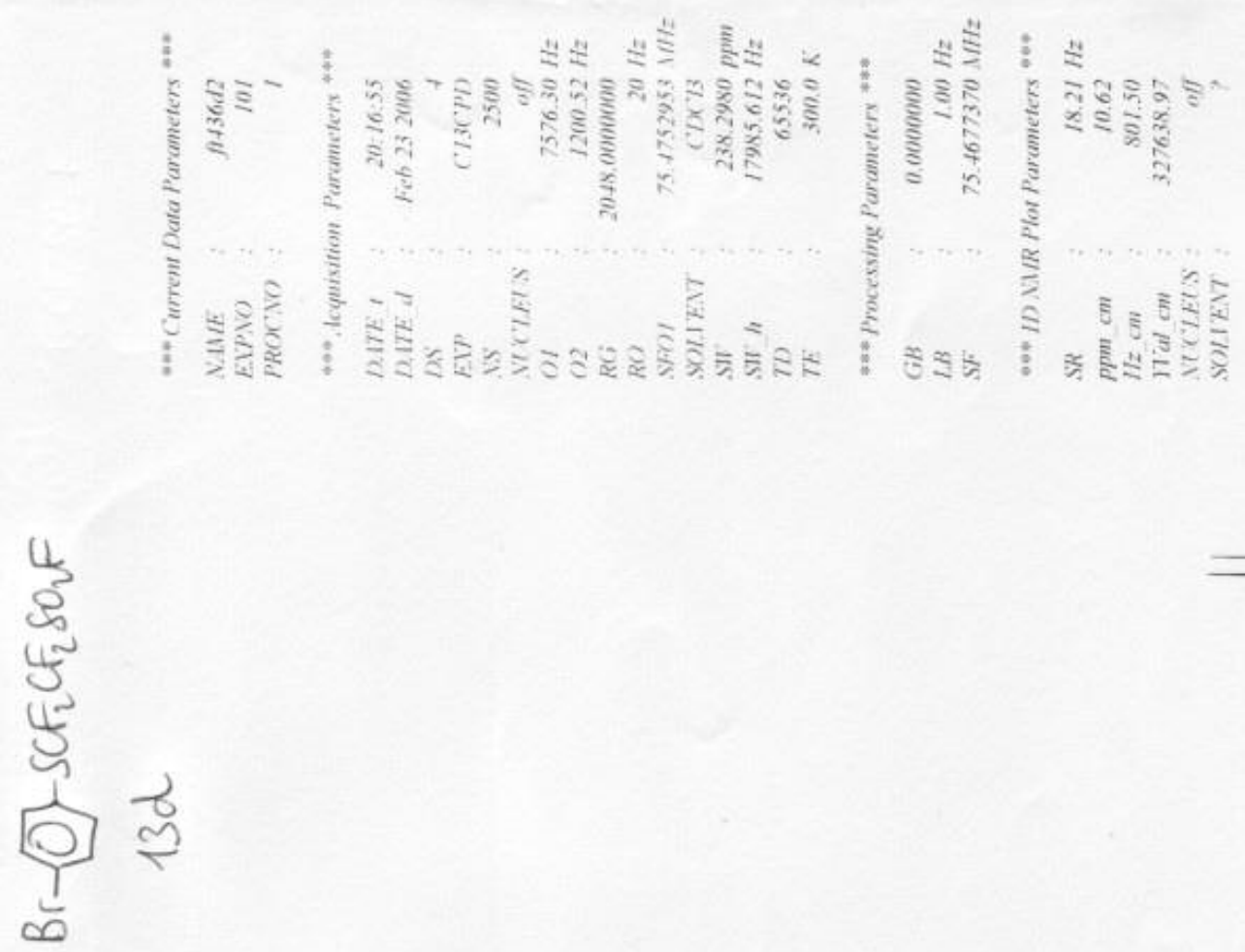

282294
$009126]$

1850721

$2850^{\circ} 721$
$2180721-1$

29607 है।

est $121-$

ZLII $721-$

ELUE $s=1-$

$2+202221-$

6r5l $281-$
Egl 8821

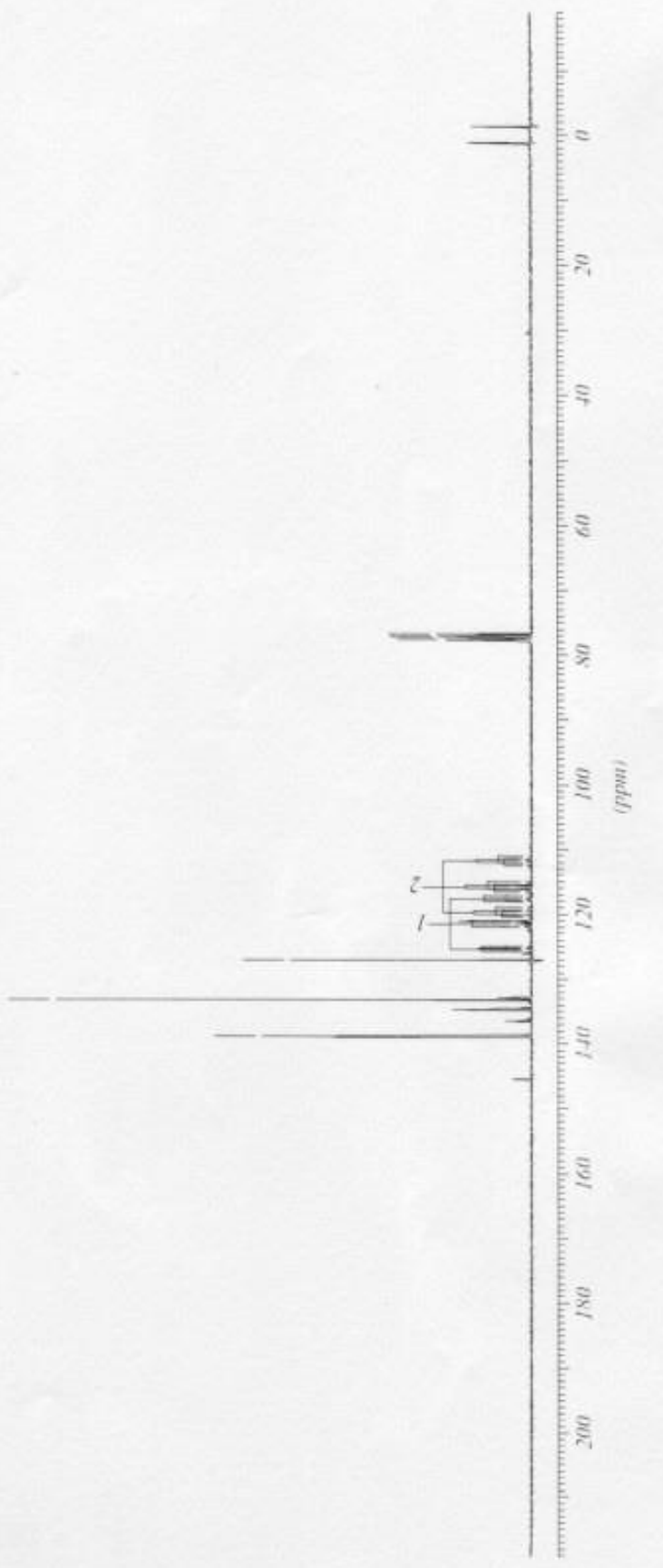




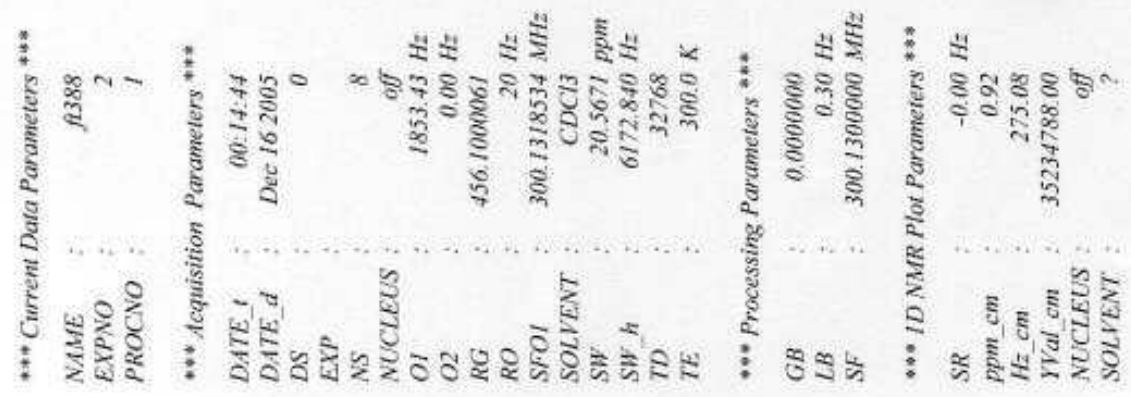

$\sum_{\substack{10\rangle \\ 3}}^{5}=$

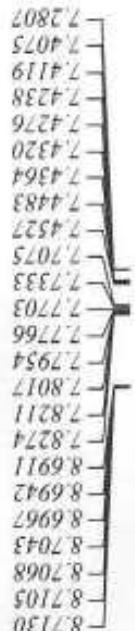

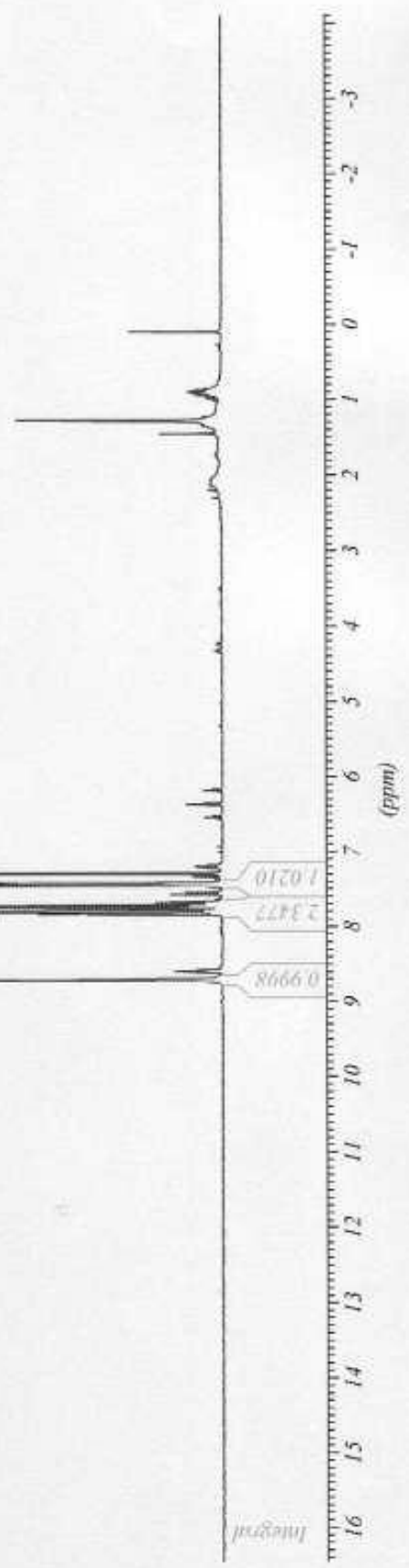



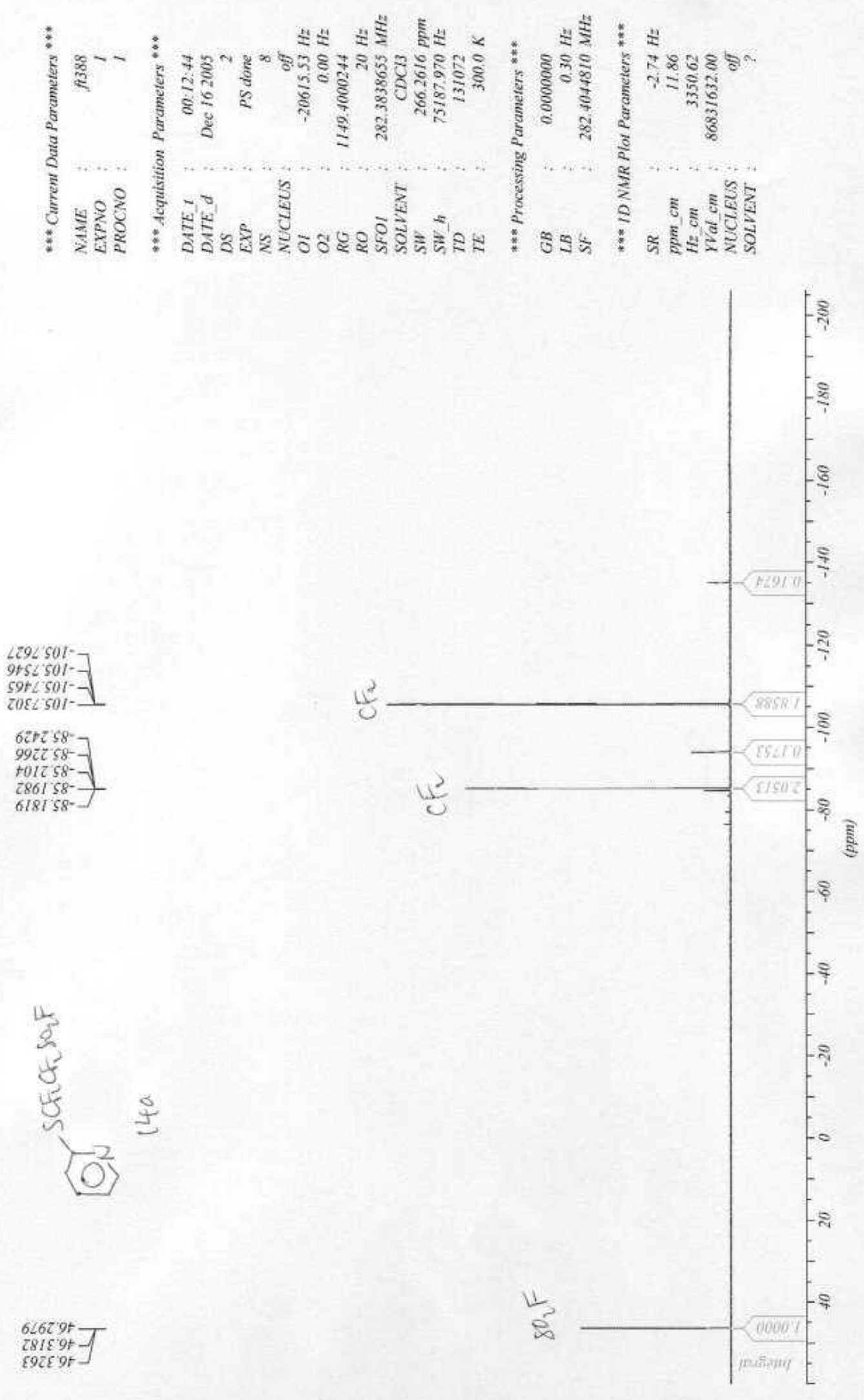


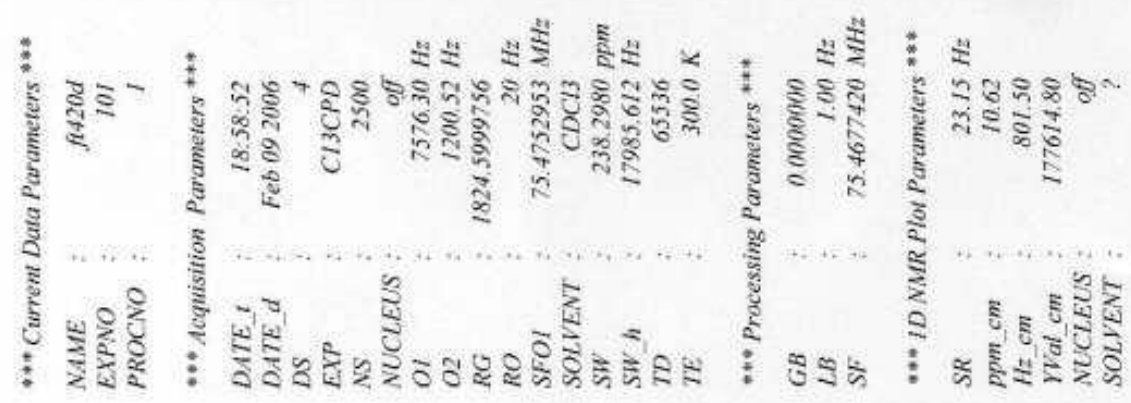

造

$00912 L-$

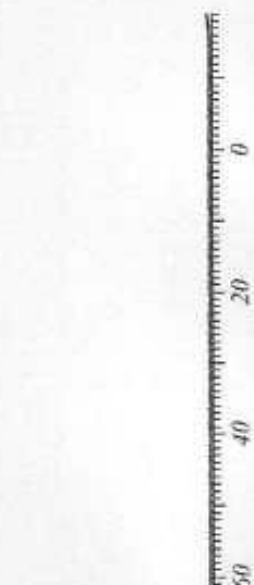

OzLLSOl-

2082901

tro $\mathrm{CH}$

दEZ2ZZI]L

stist

$\angle E O T S Z 1-$

$6125921-1$

$26+0821$

$626082 \mathrm{I}$.

$8 E 92621$ -

$4 s+7 \varepsilon l-$

$5 \angle 9+T E l-[$

$\varepsilon 296 \angle \varepsilon t$

c59t $951-$

$062+951-$

$180595+1-$

L2z59t1-

$\angle I 55971-$

c9959+1-

$6 L+0 \leq l-$

$8201751=$ 


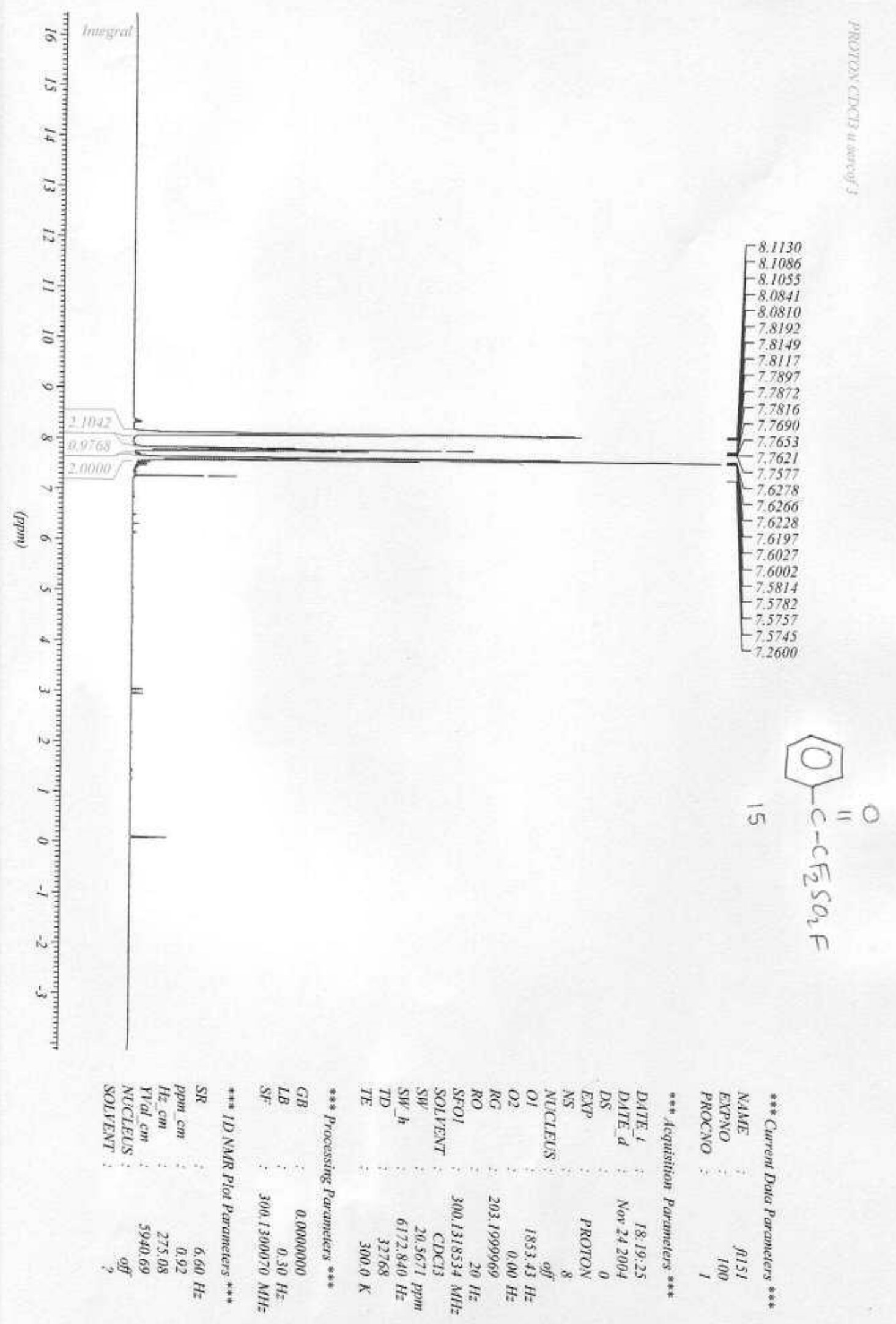



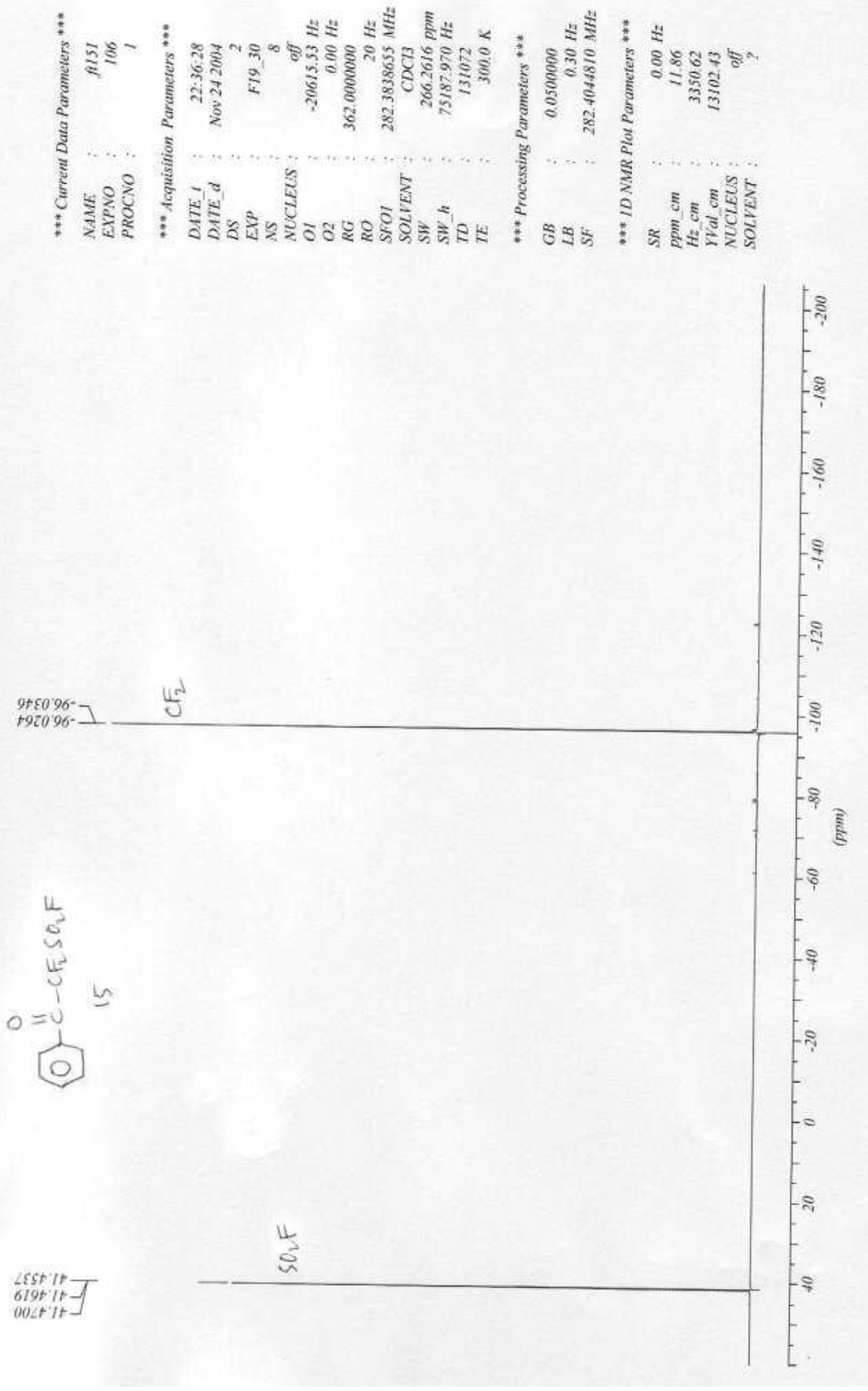


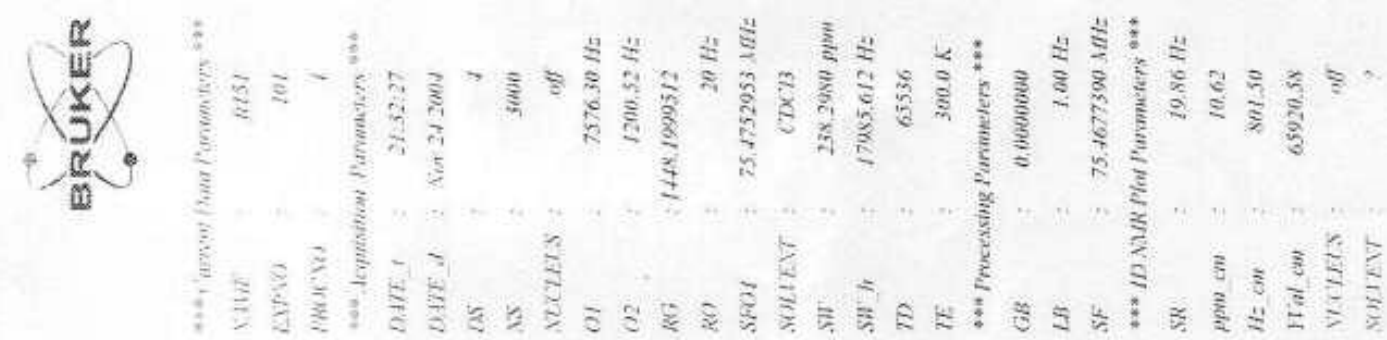

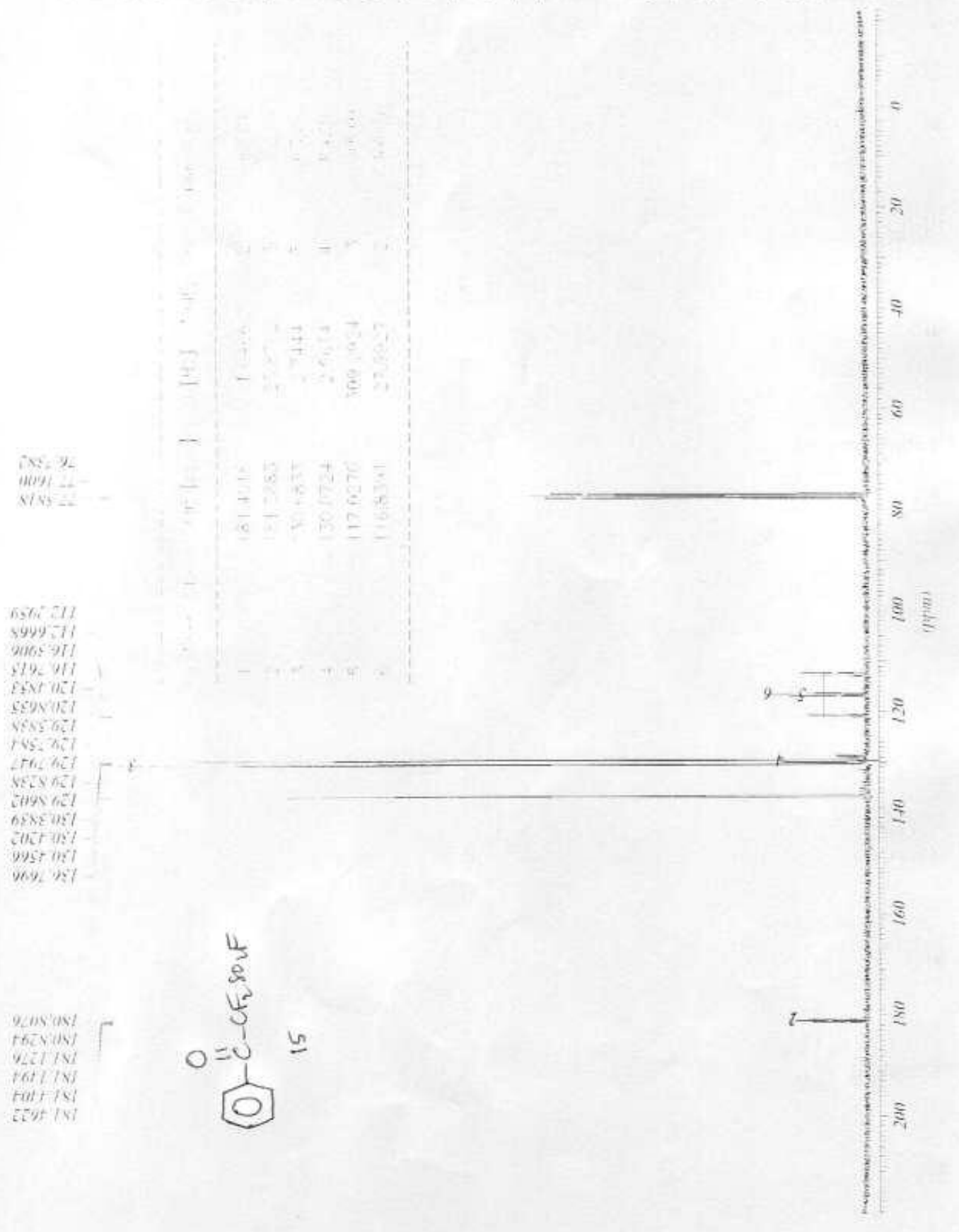



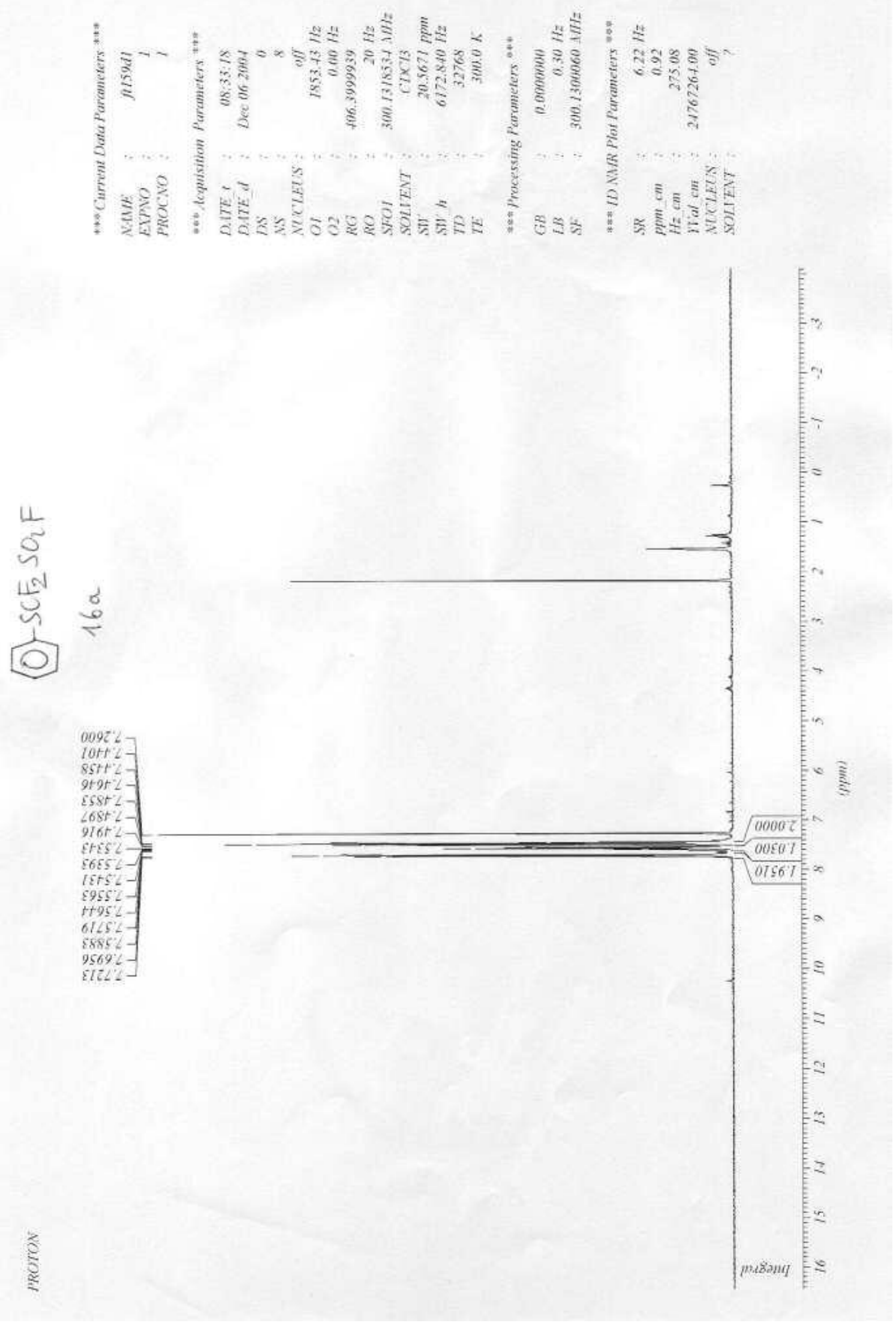


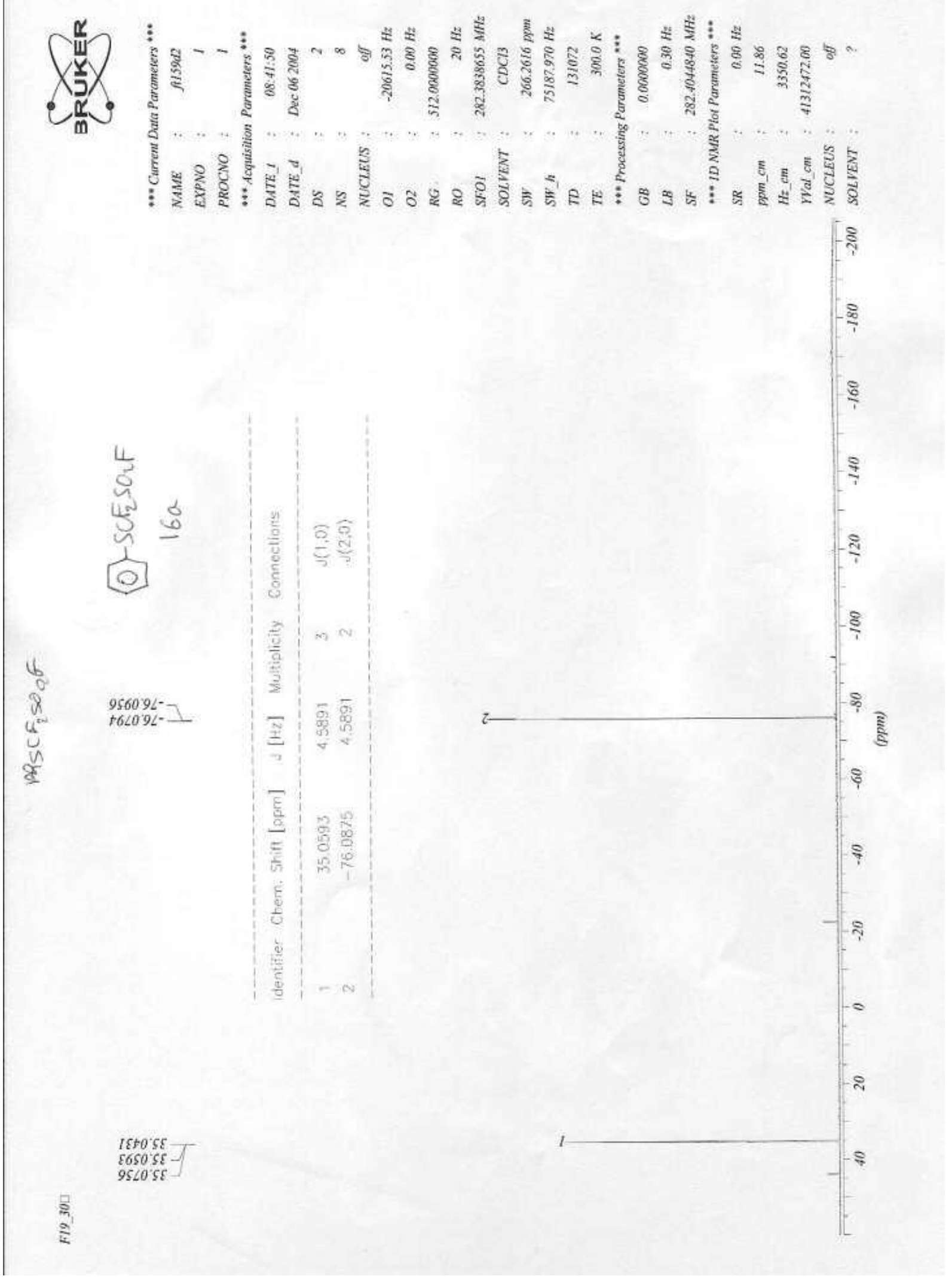




$$
7
$$




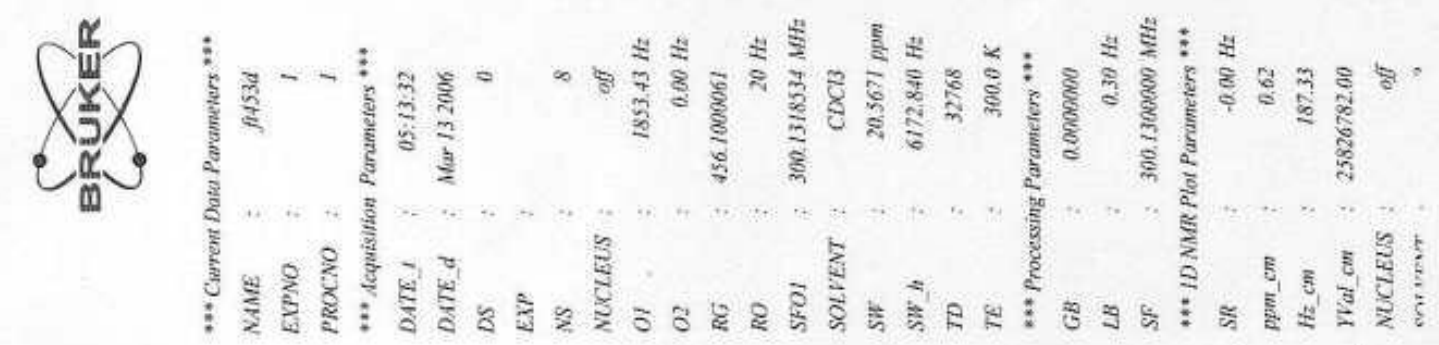

$\prod_{1}^{11}$

$2 \operatorname{css} 2$

+8812
$8 Z 12 \mathrm{~L}$

t18E 2

$5202:-$

56162

ozeL 2

$06+22=$

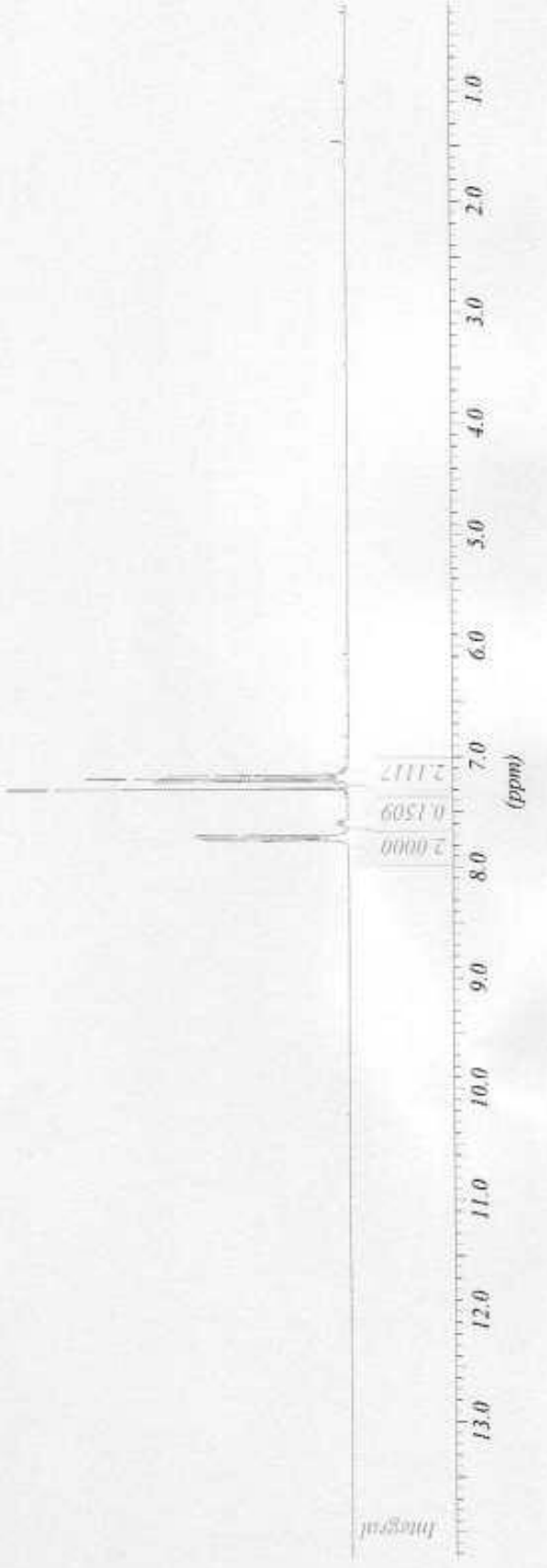



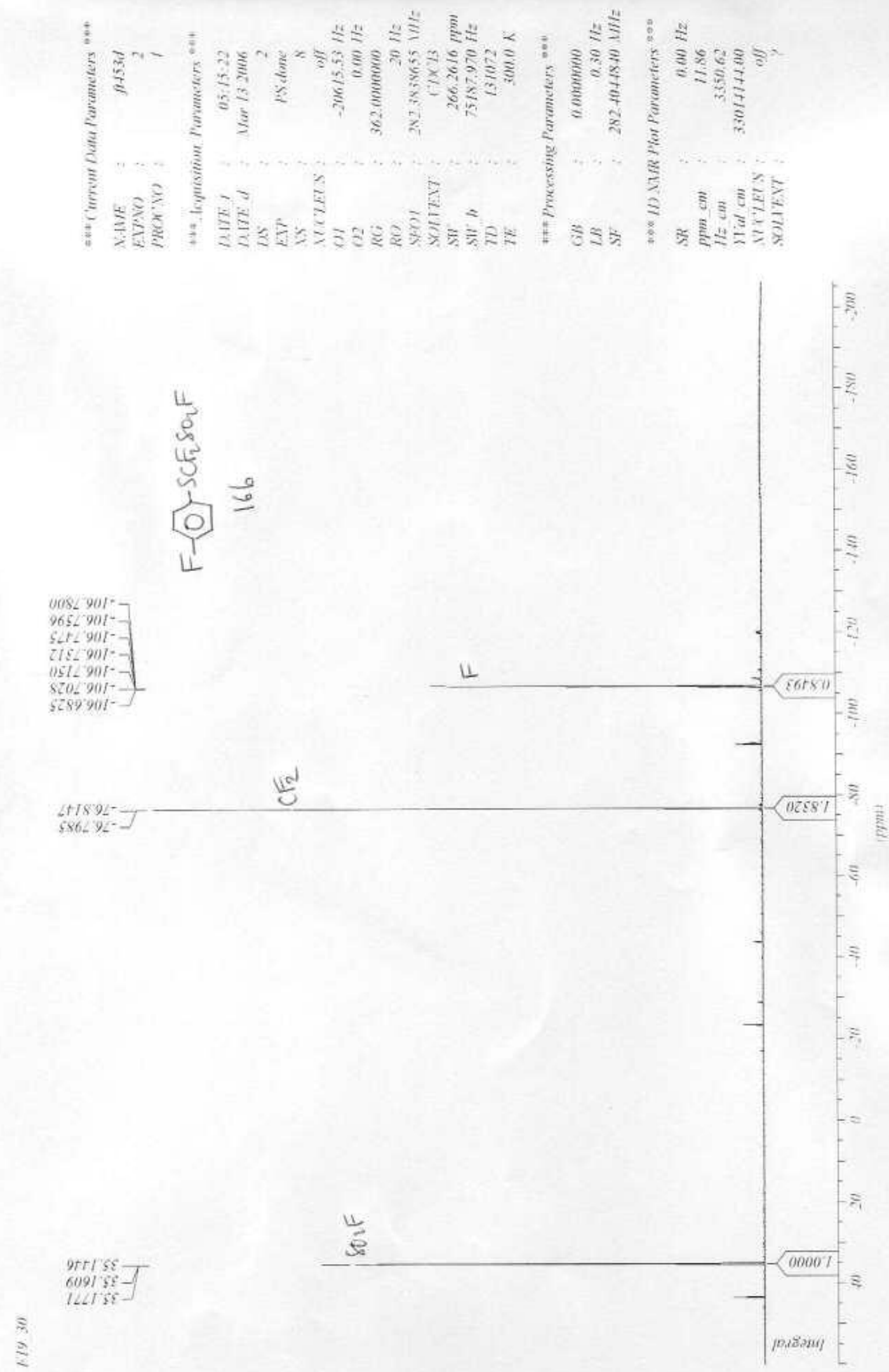


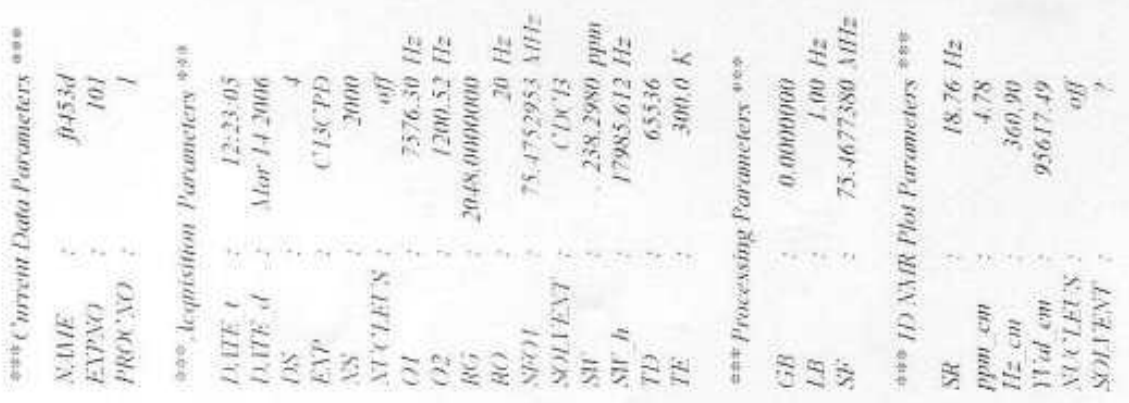

(1) 1212

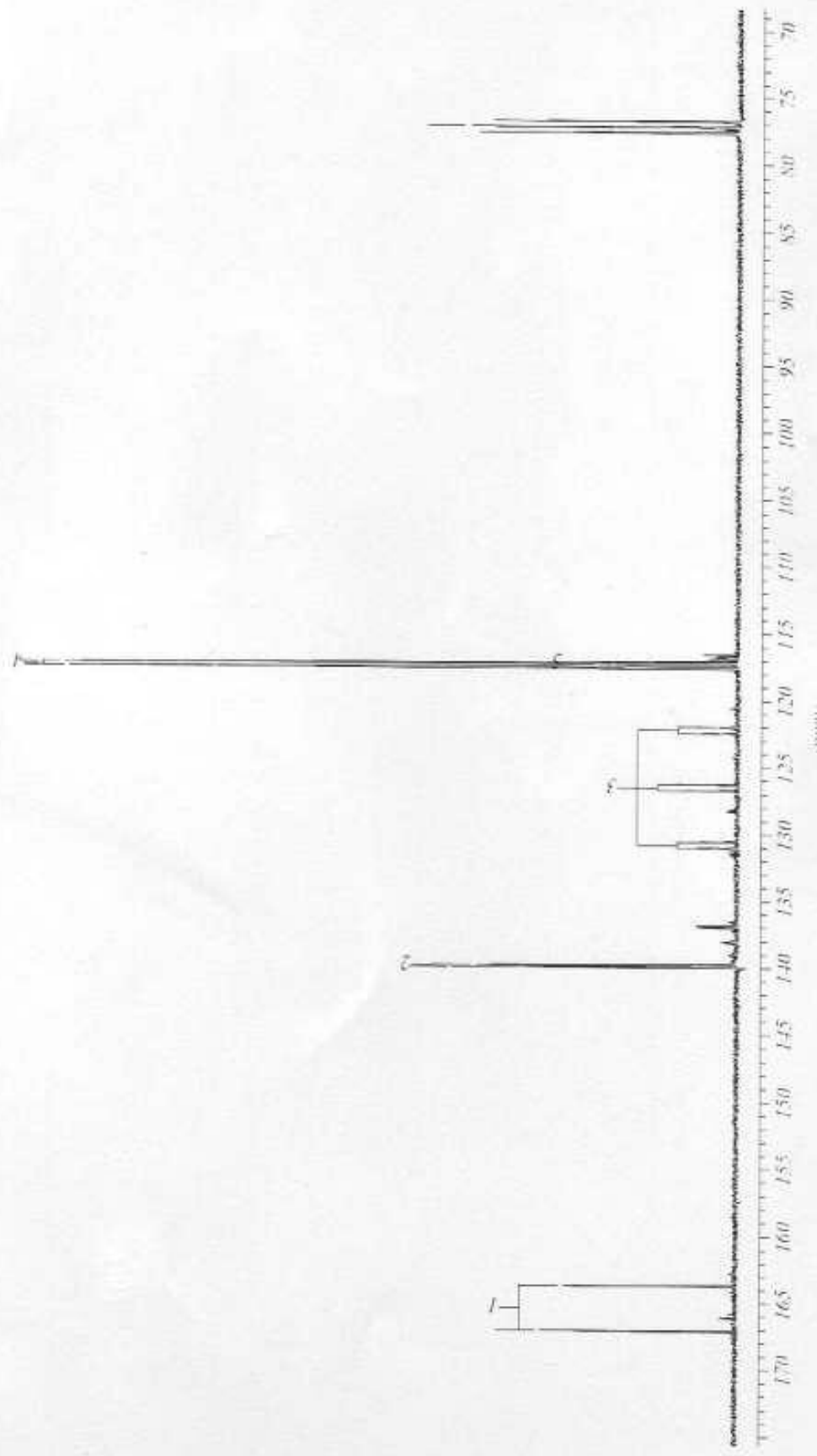

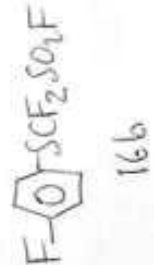

2612:11

$|56265|-$

$5816681]$

cergest -

$0870<91-$ 


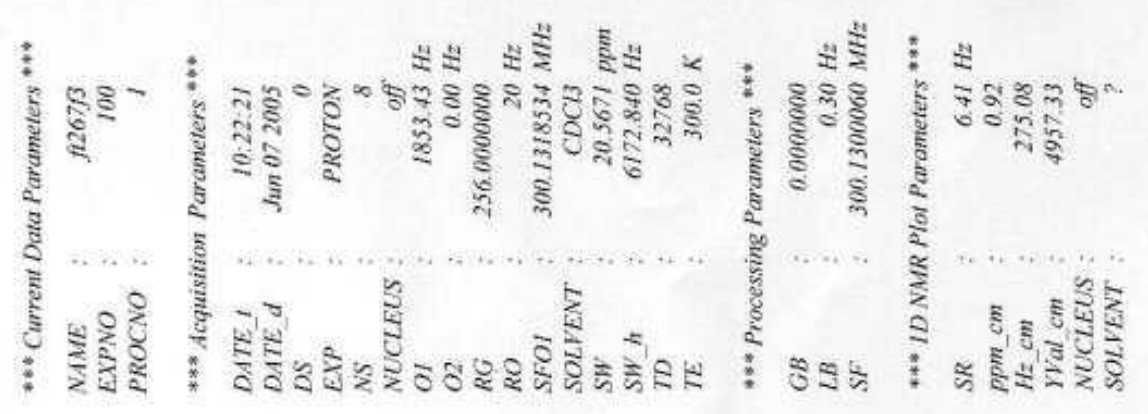

告

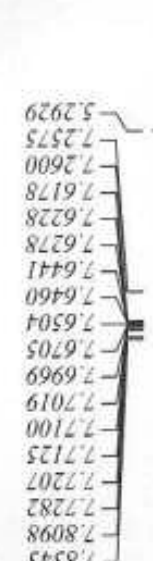

$\frac{8}{5}$

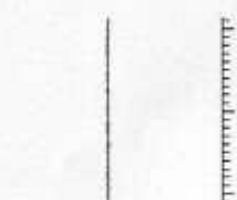



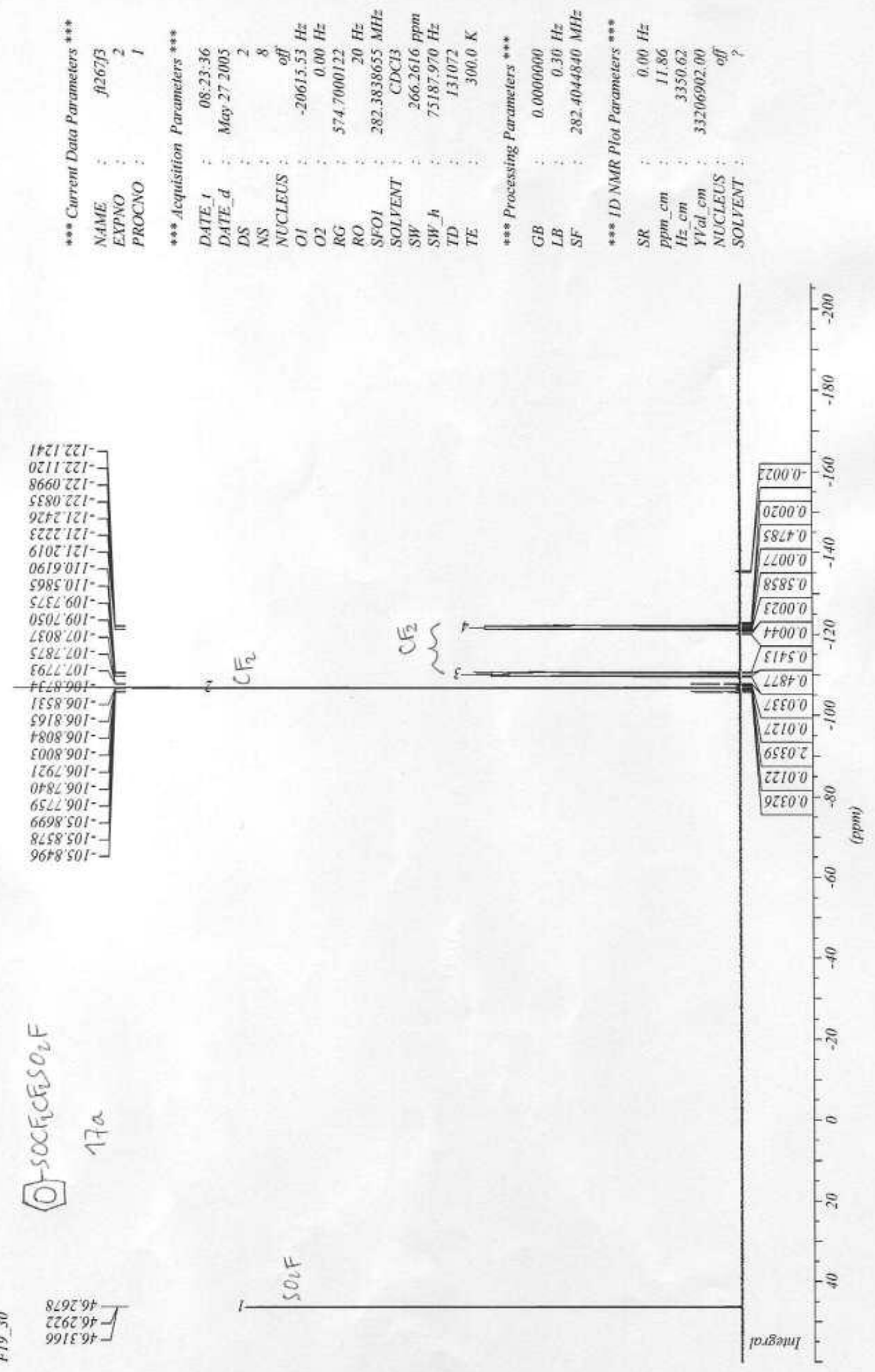

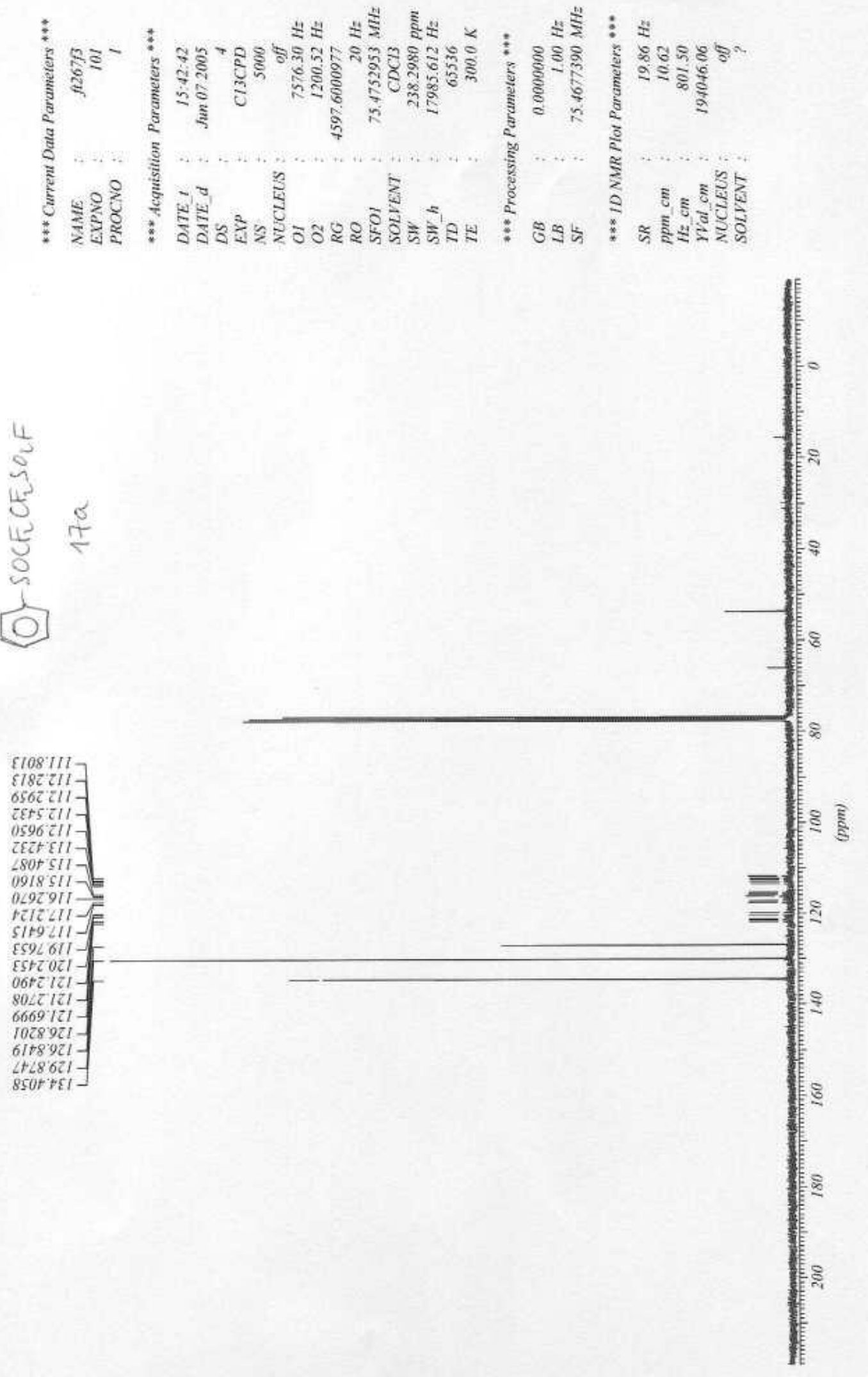

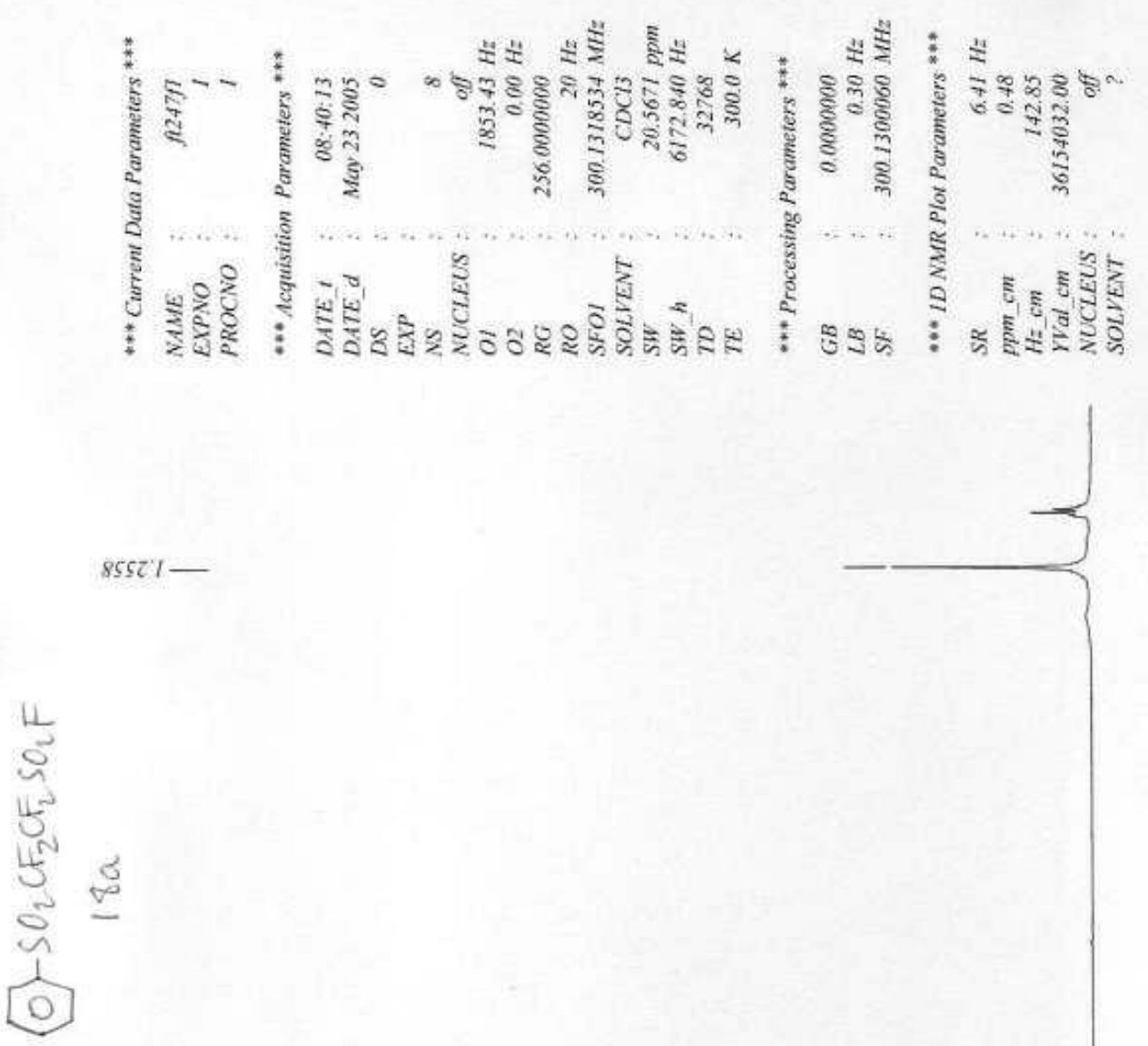

$8+6 z \varsigma-$

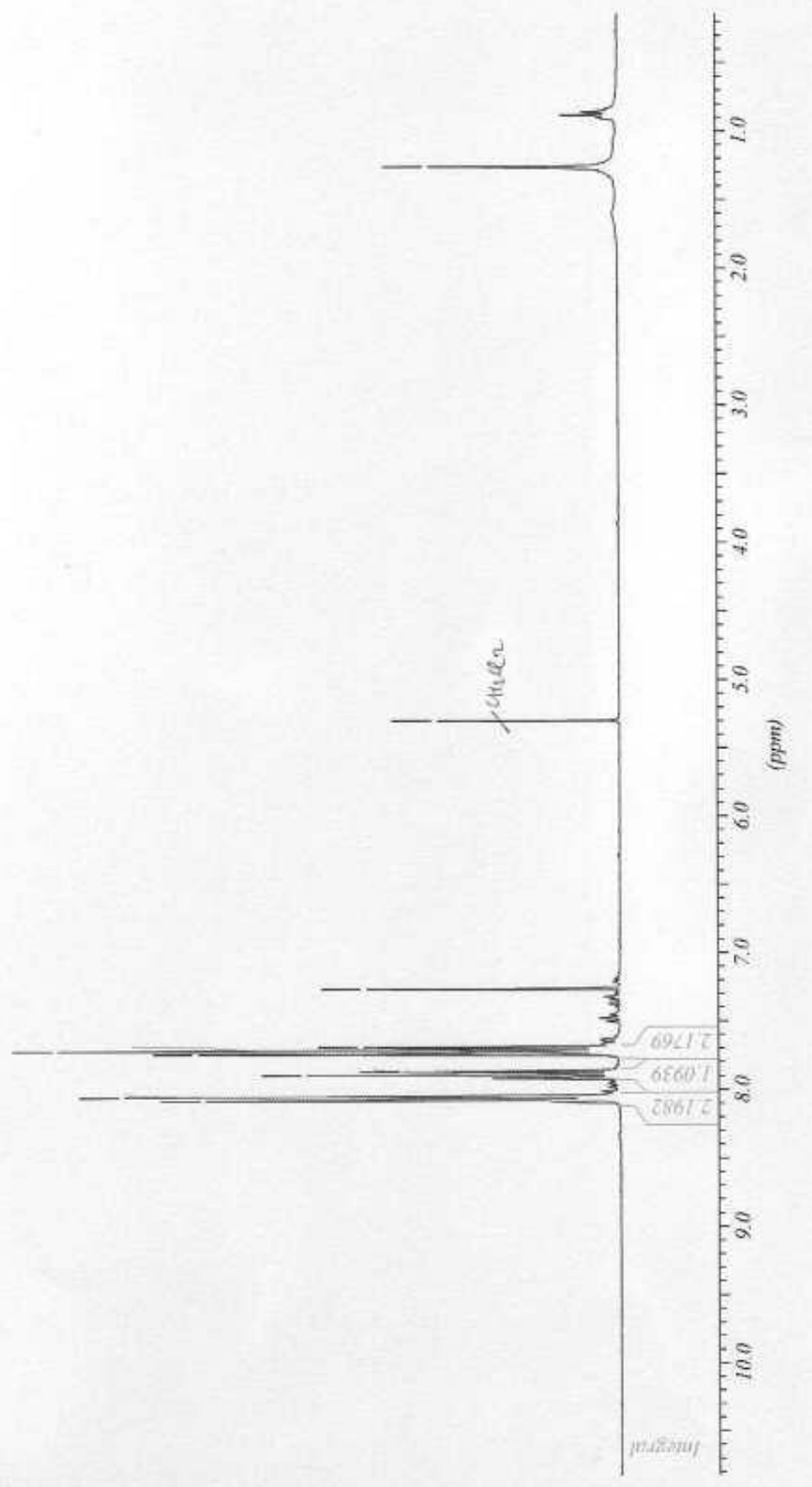



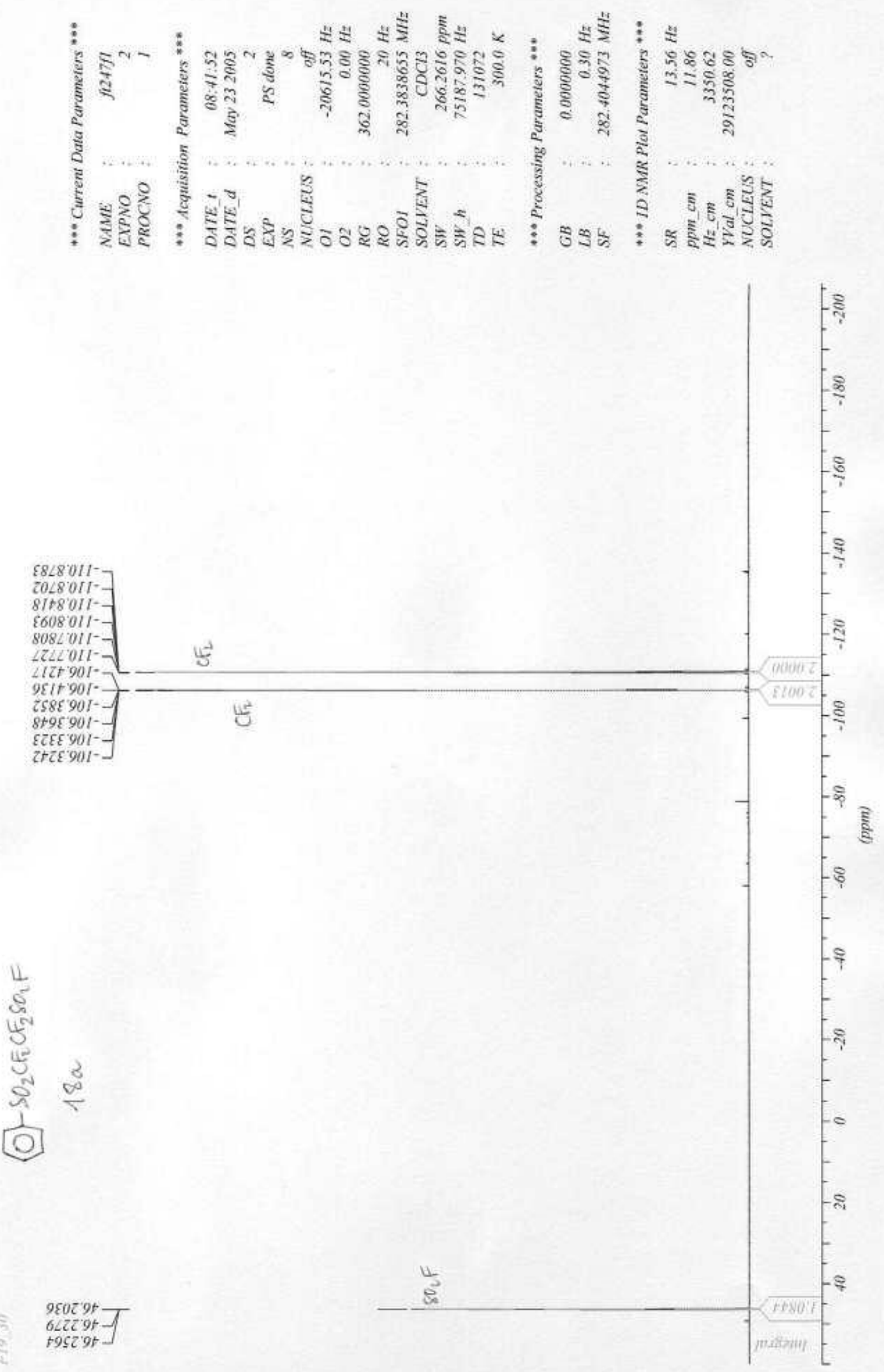

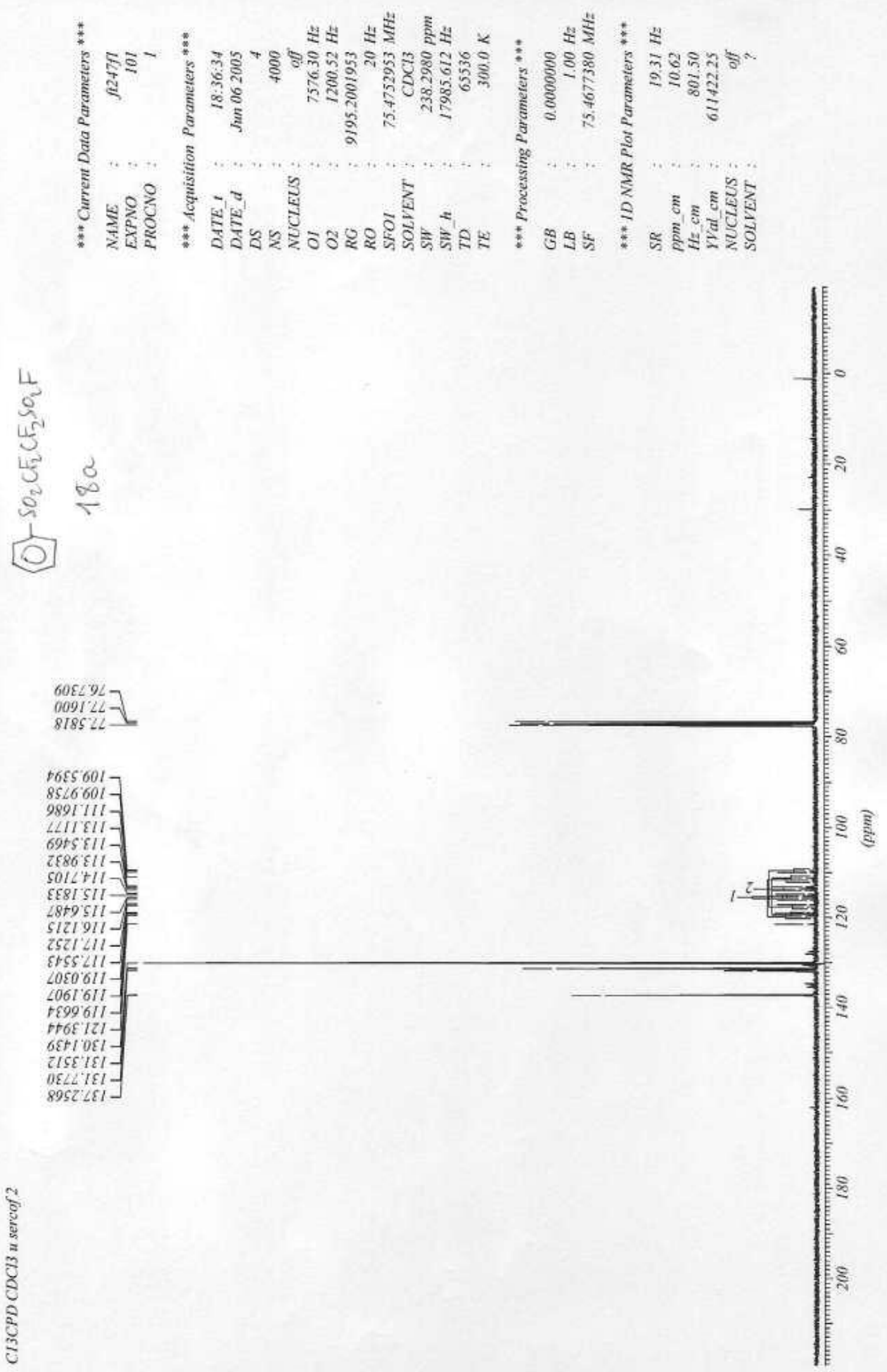


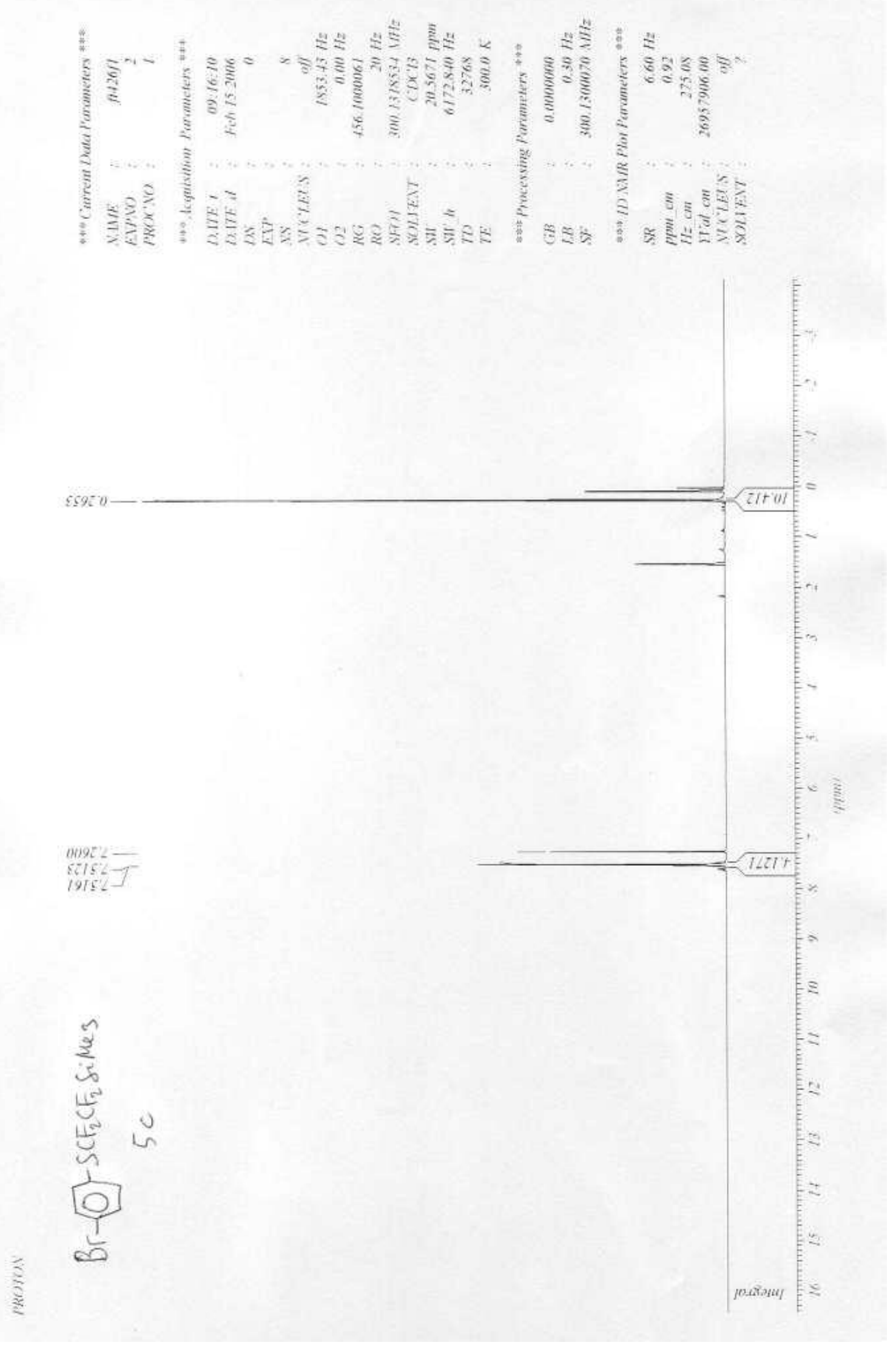



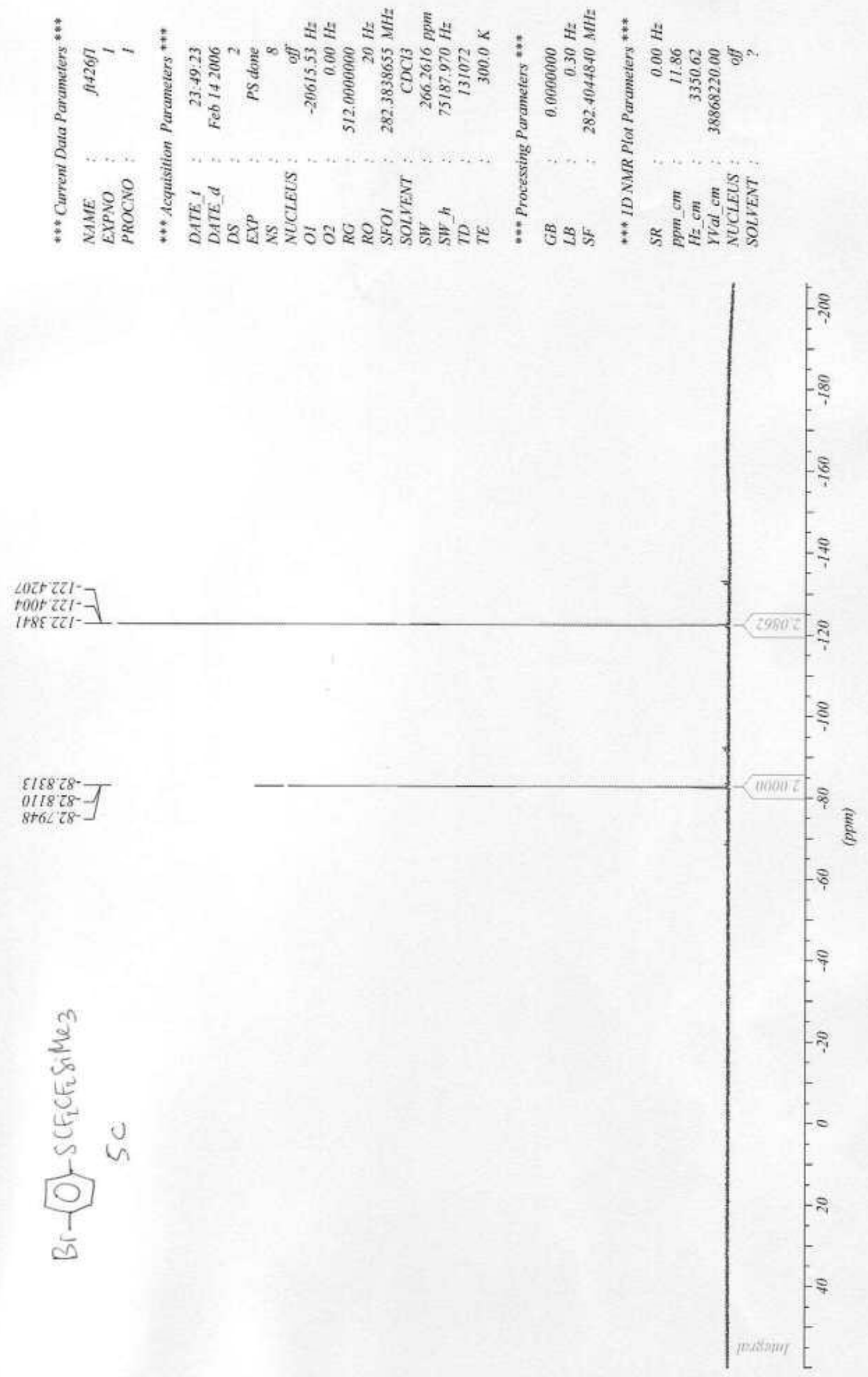


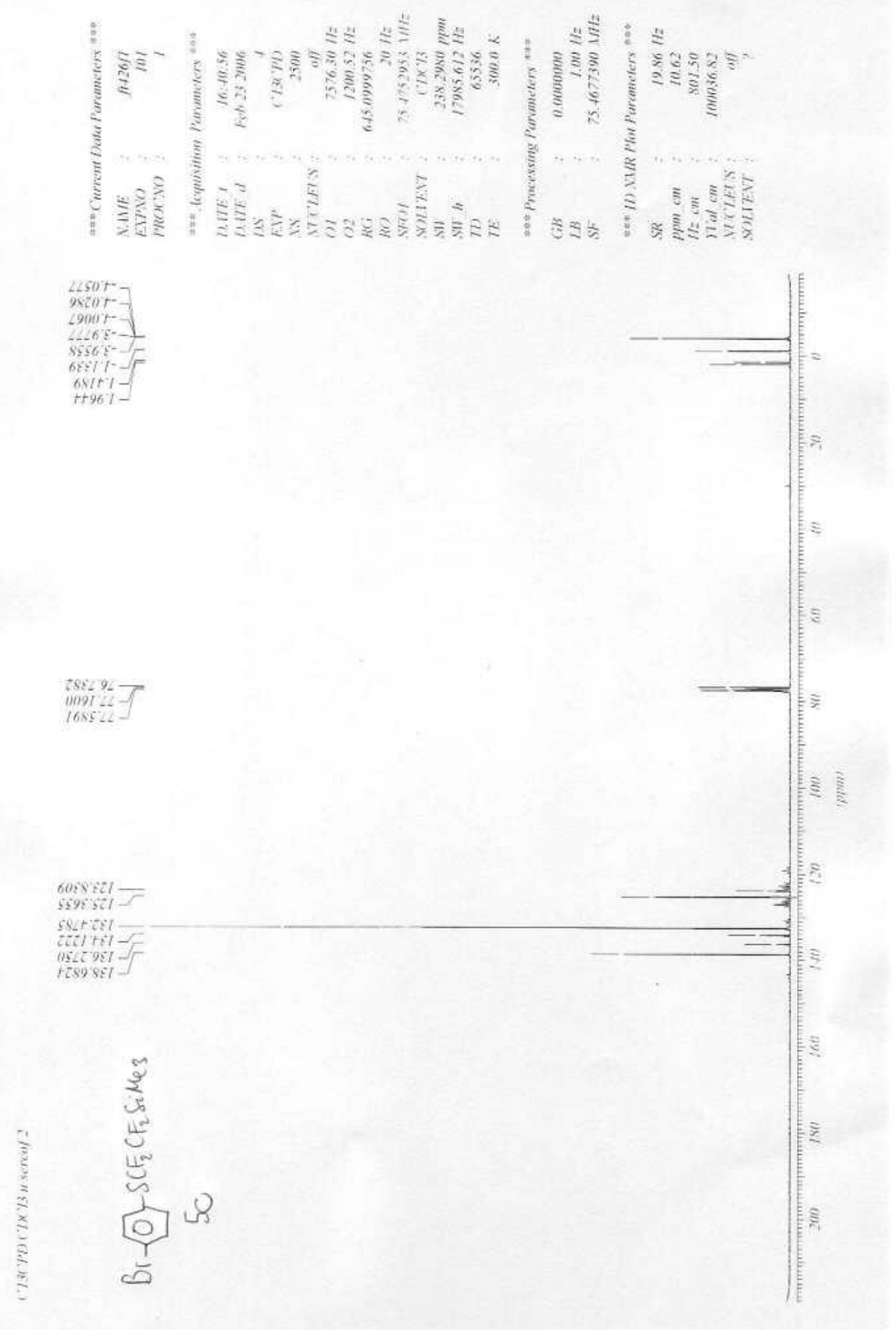



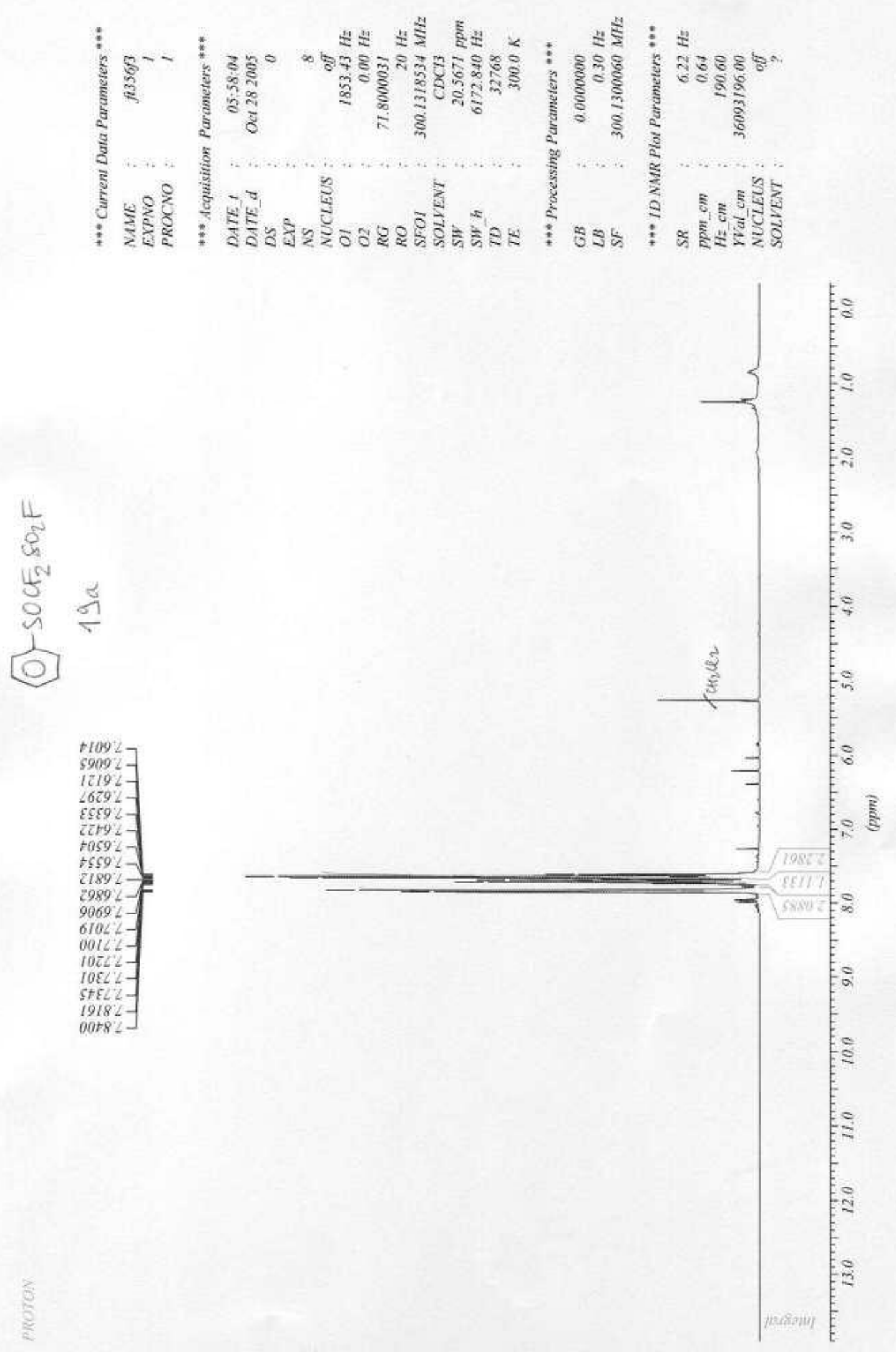

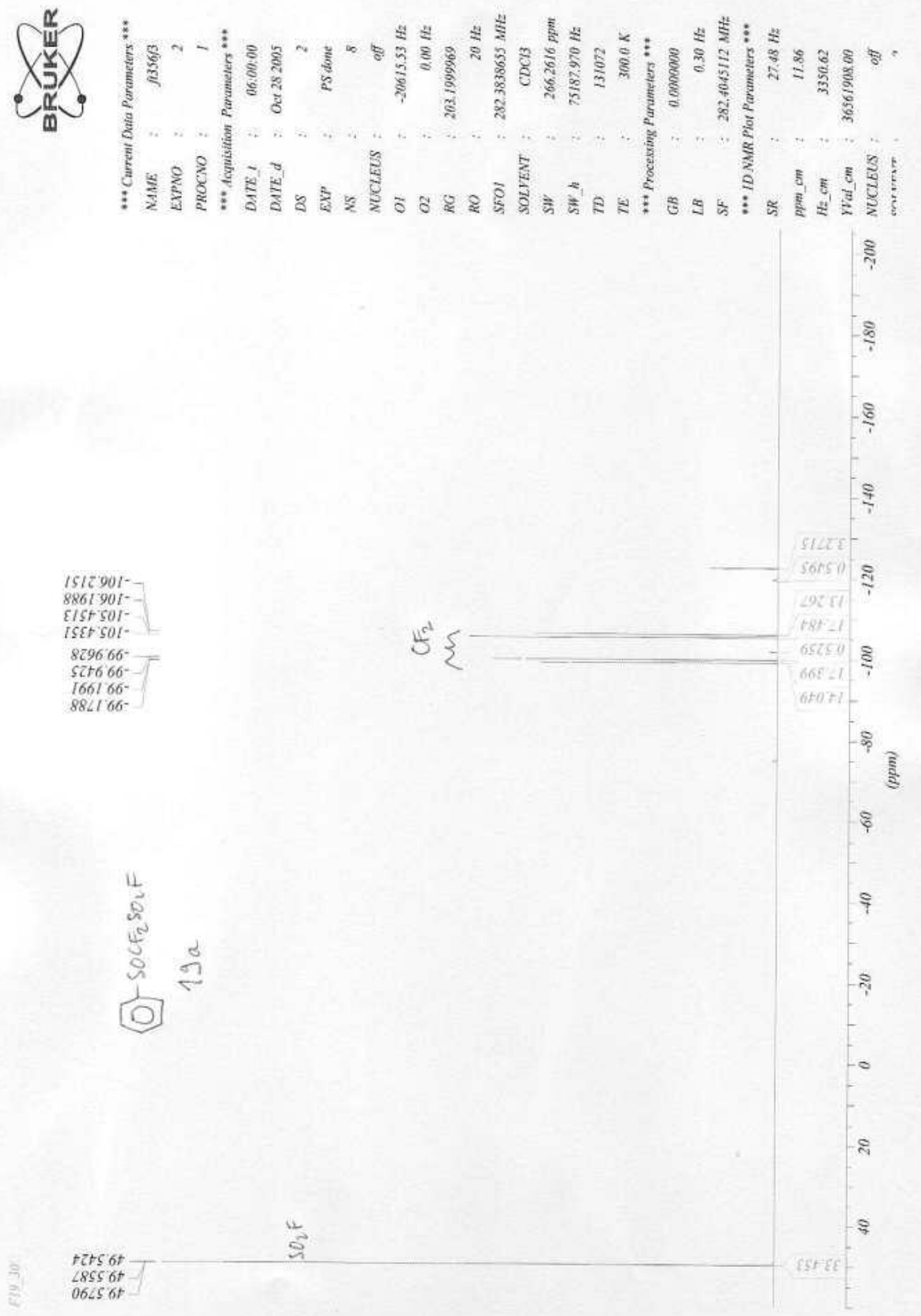


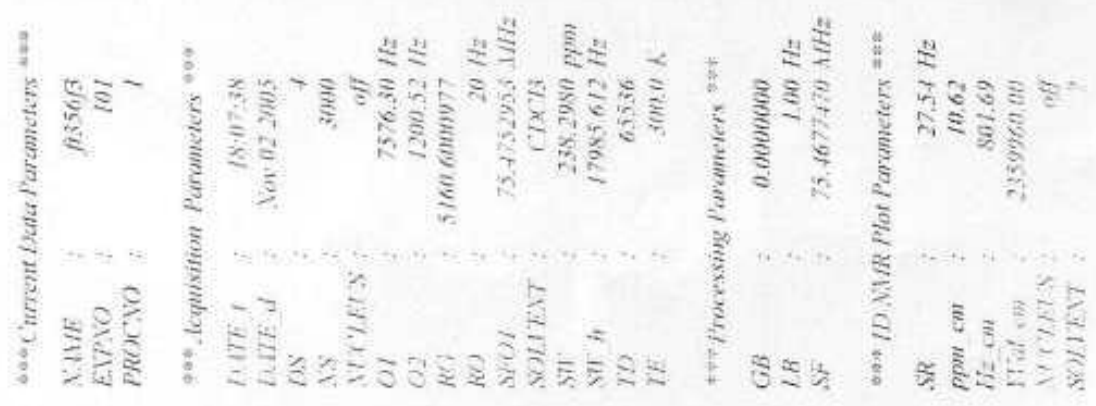

$\frac{1}{5}$
$\frac{5}{5}$
0
0
0
0

po9r6L-

19/06ll-

HEF $611-$

sitio:

megsizl

$1696281-$

6109021

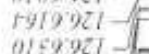

0IS] SEI -

$95 \mathrm{x}-821-$

$1116621-$

at $90+5=$

[260t-s]

gegrs

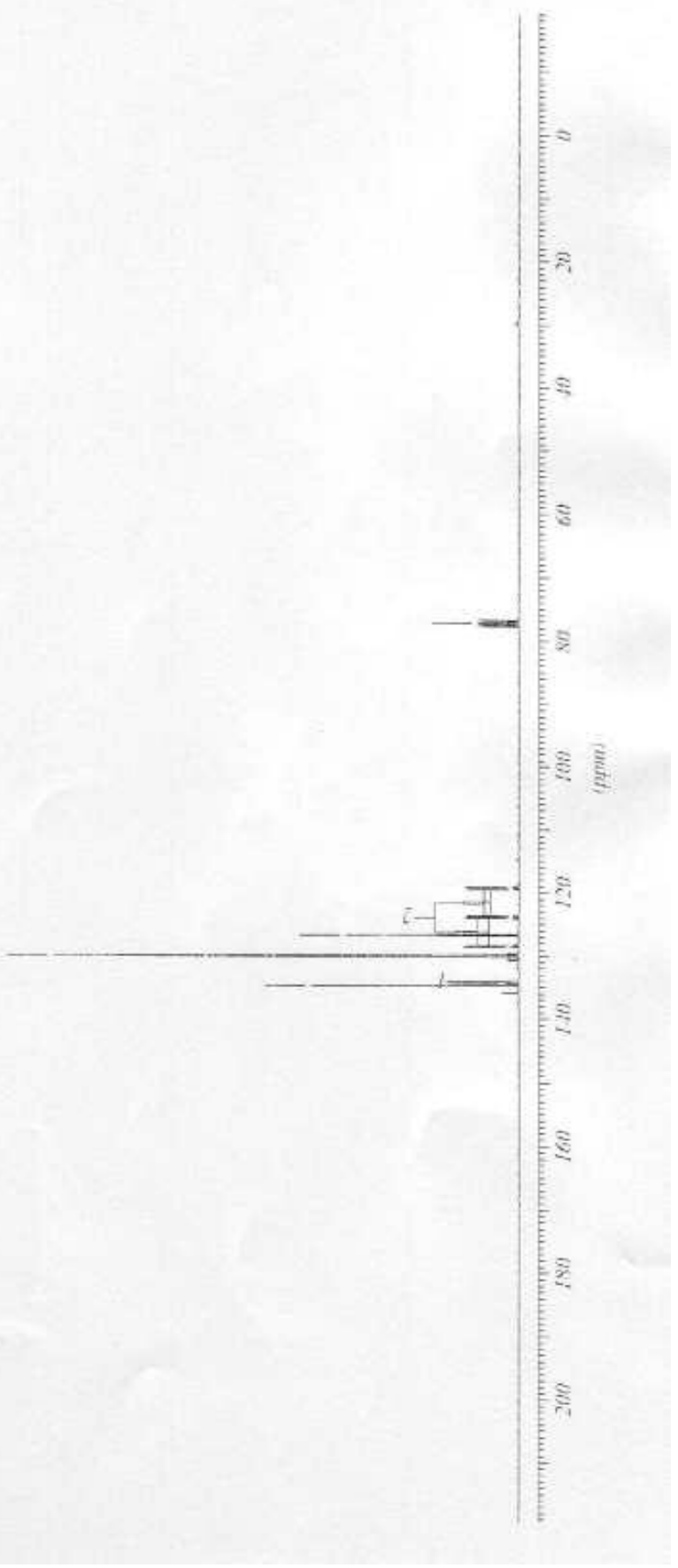




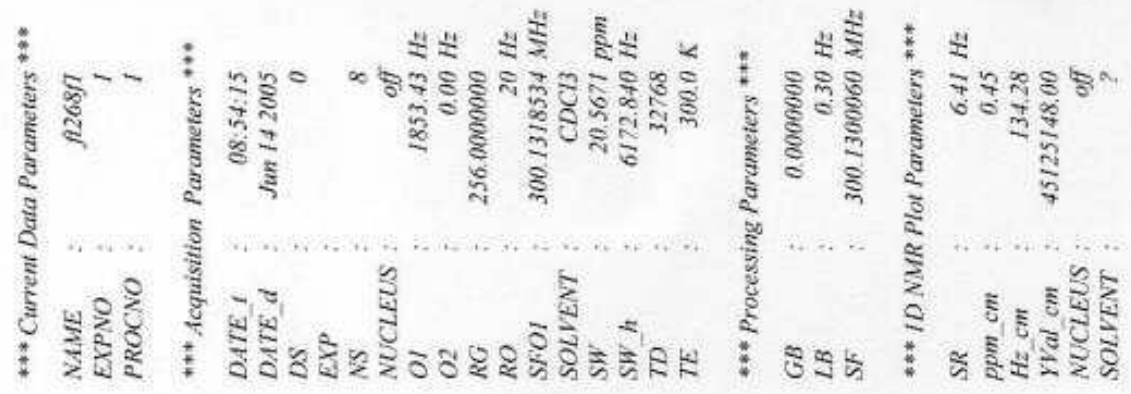

$\frac{1}{3}$
$\pi$
4
0
$\infty$
0

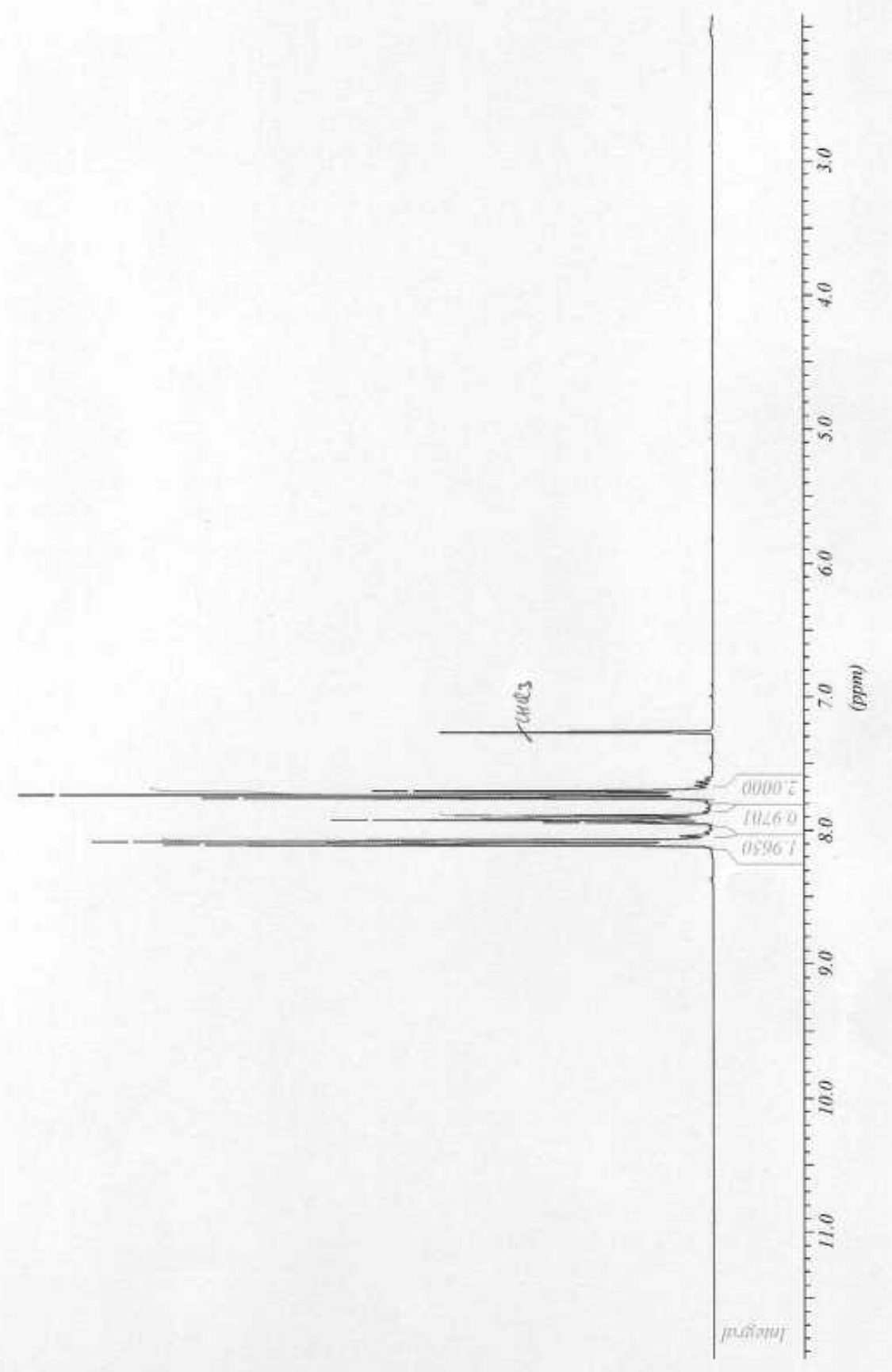


第

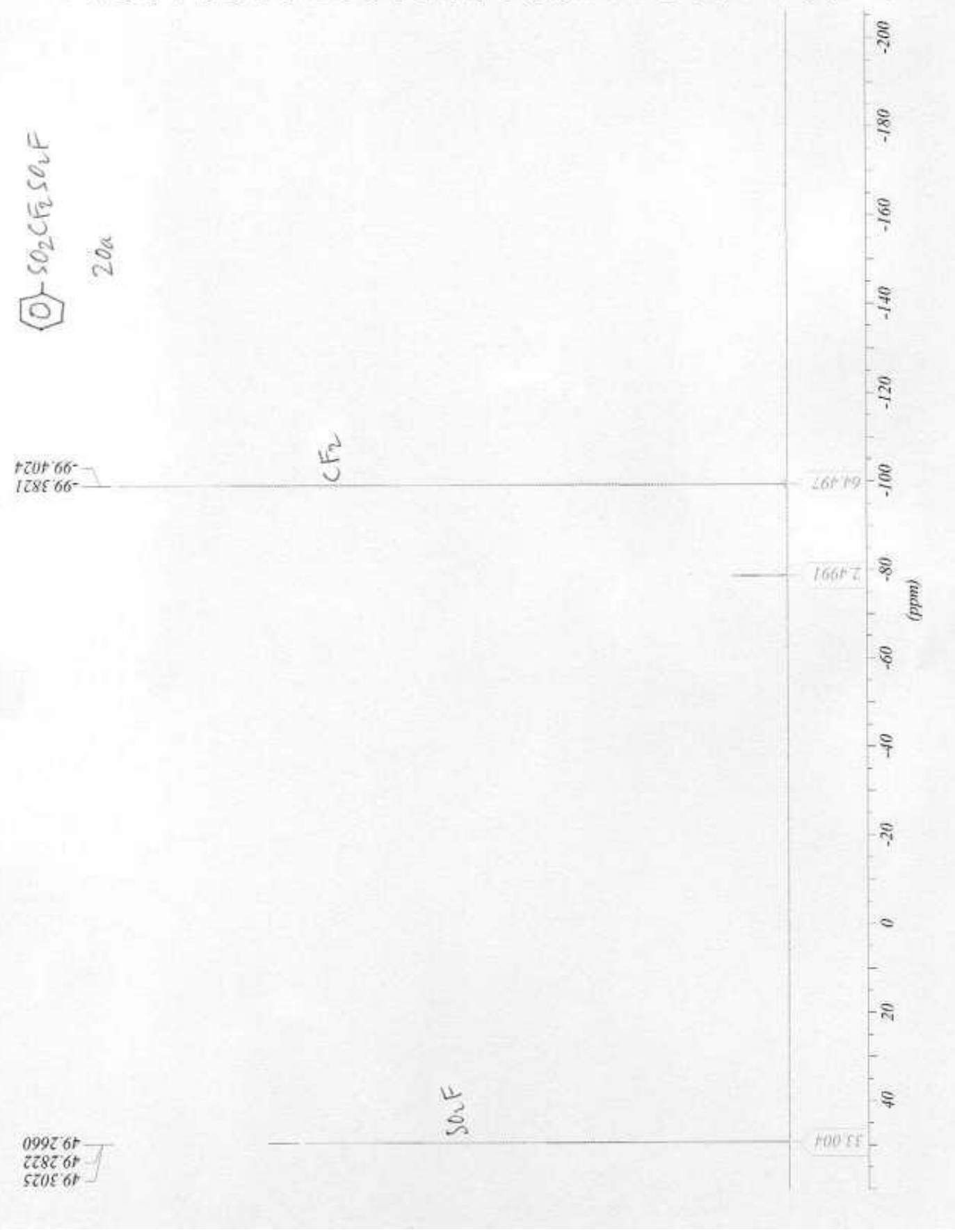




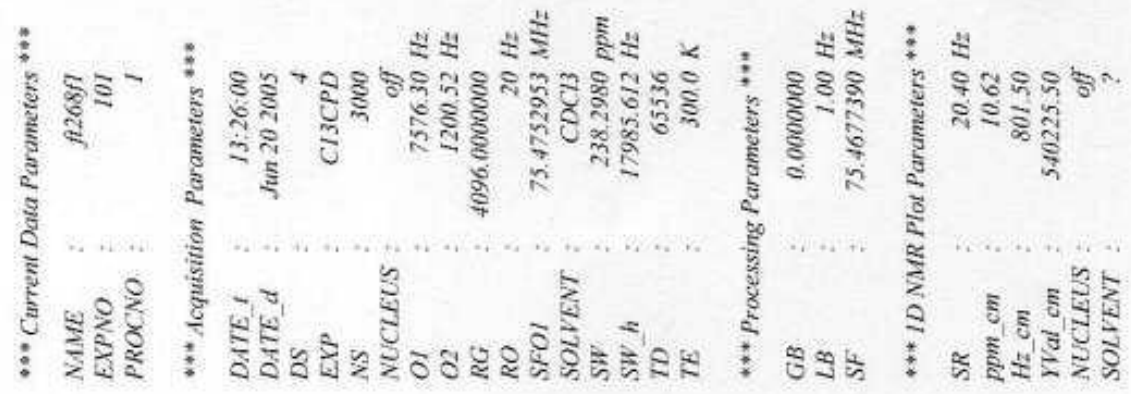

$\overbrace{0}^{14}$ o

$28 \varepsilon 29 L$

168521

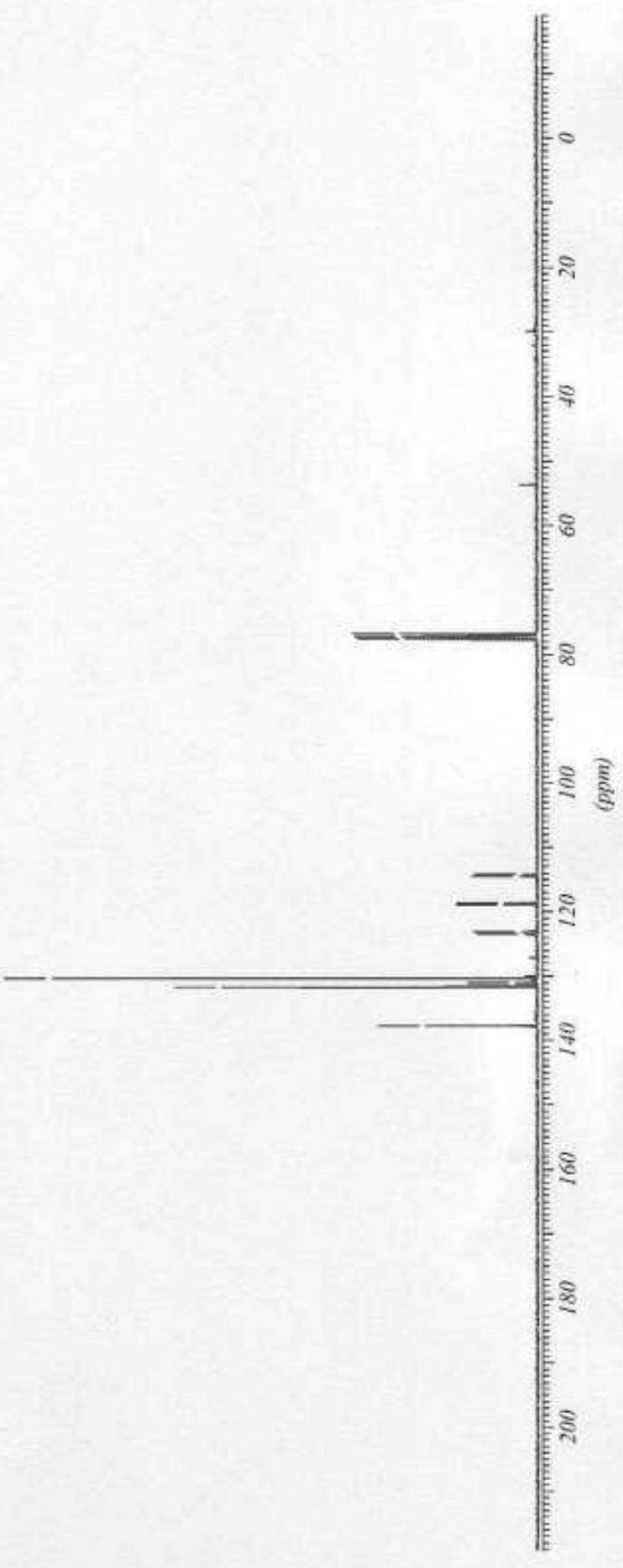




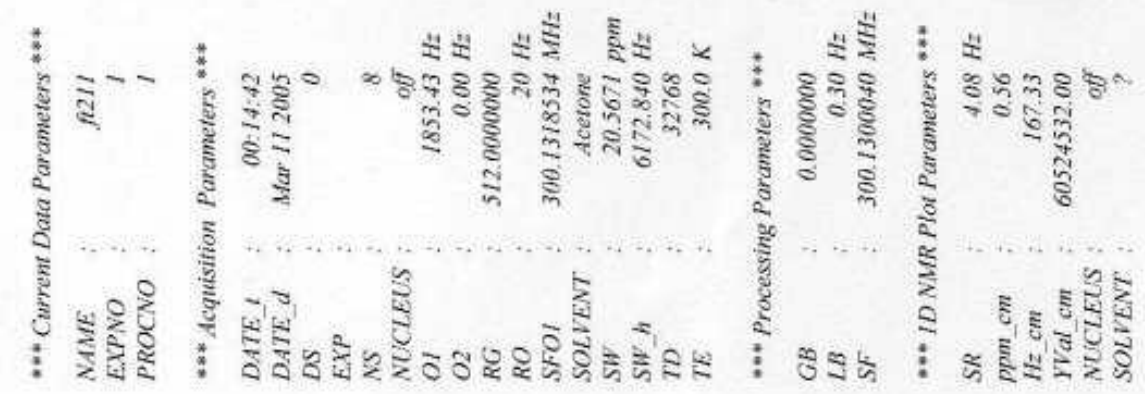

00502

$8511 \%]$

16Et 2

$09 T^{2}-$
$\mathrm{C}$

$1190^{2}-$

$989+2$

$9 E \angle D^{\circ}-2$
$+\angle 8+2$

Sz6t:-

$6105:$

$520 S^{\circ}$

ZEIS

हारS-

Lors 2

$20952-$

29792

61592

$8 E \angle 92-$
$9 \angle \angle 92-$

$\oplus$

(1)

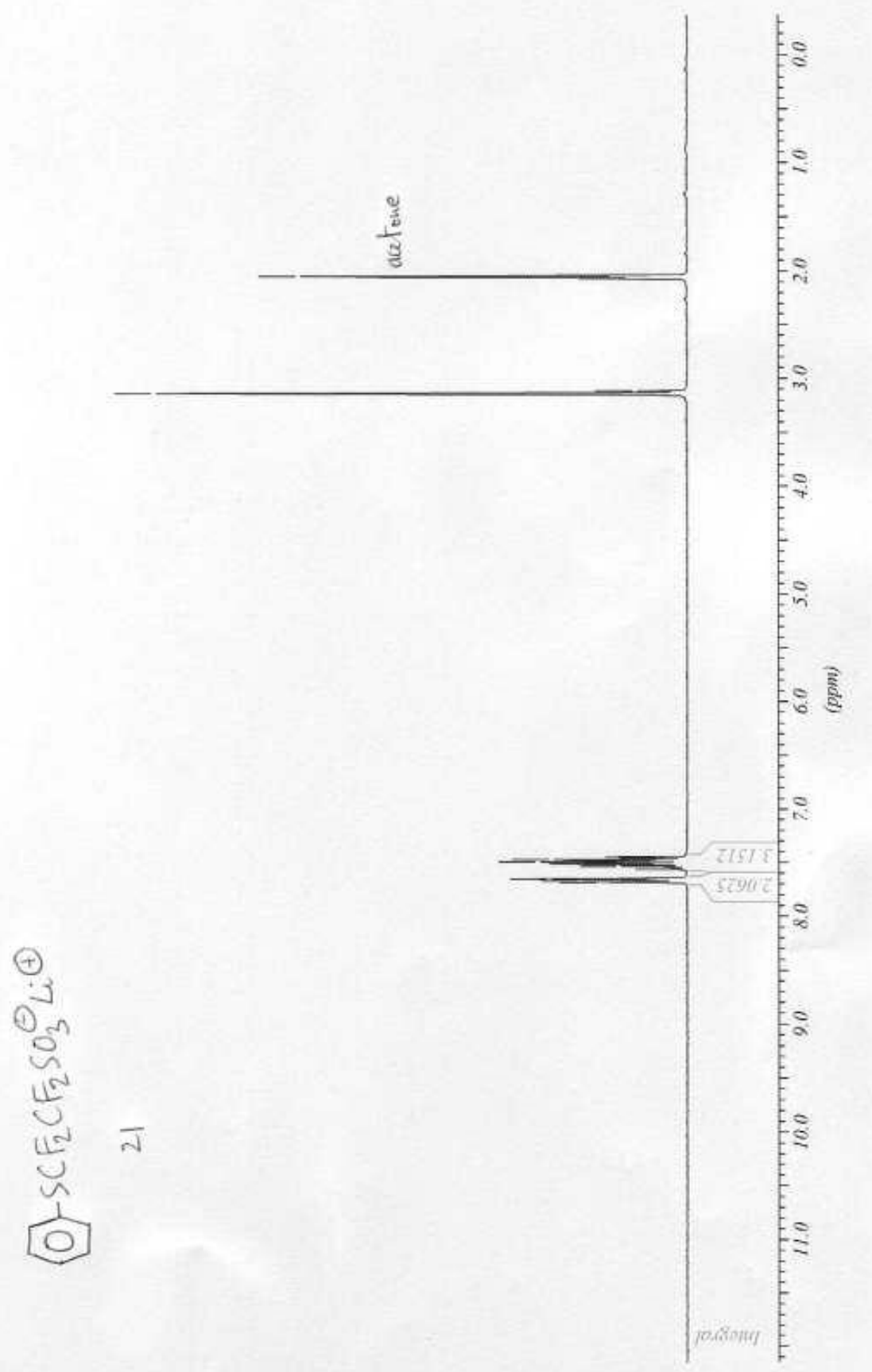




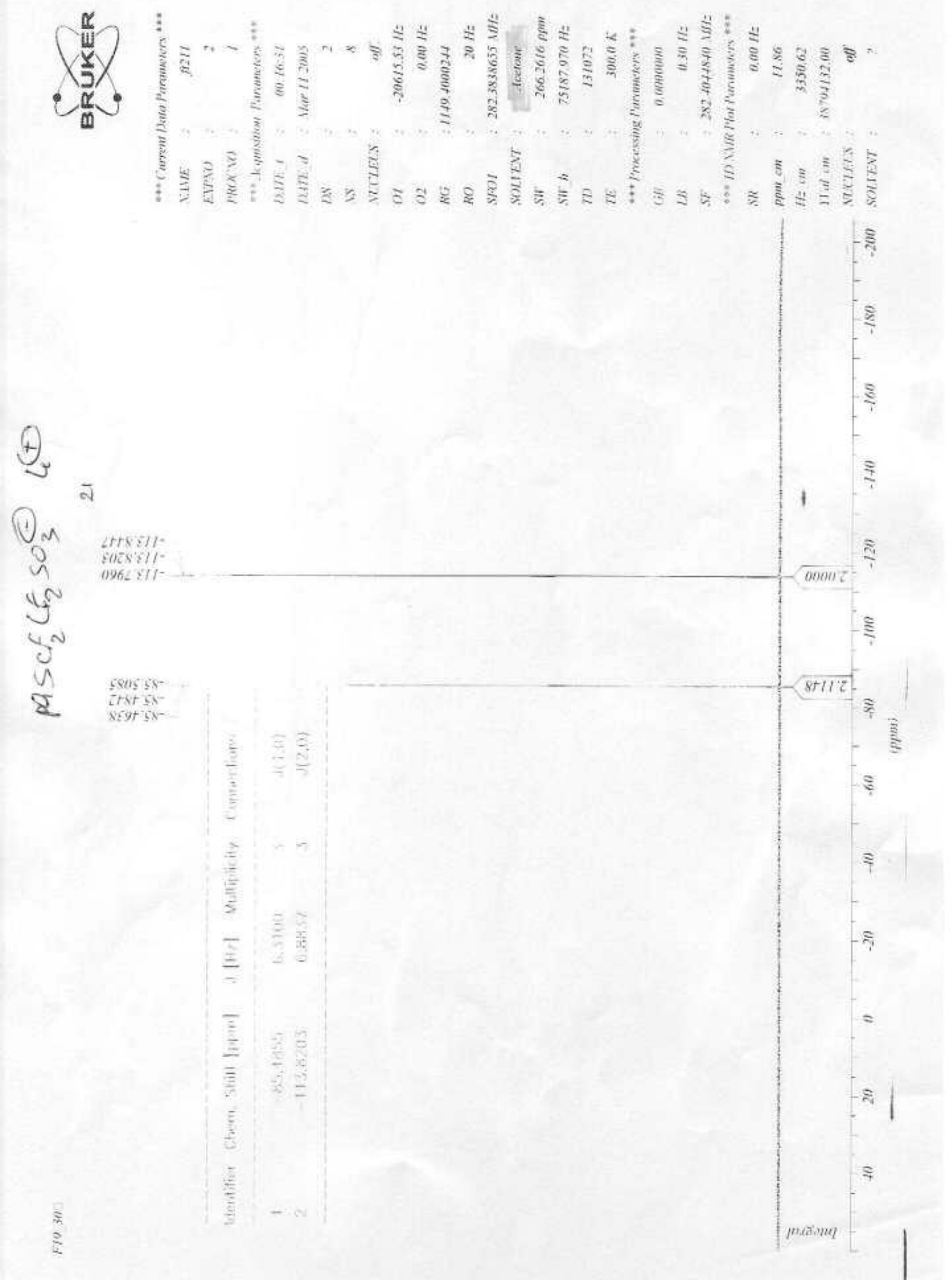




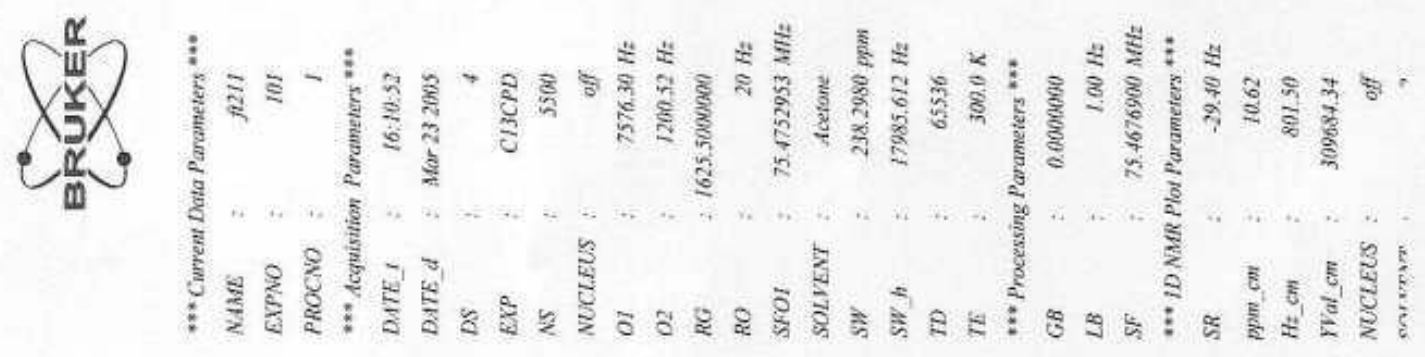

$28 \angle 562$
$00+862-$
976005

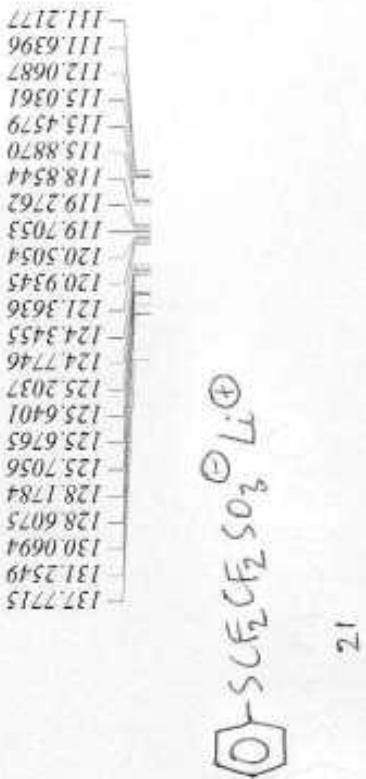

$\$ 256902$ 


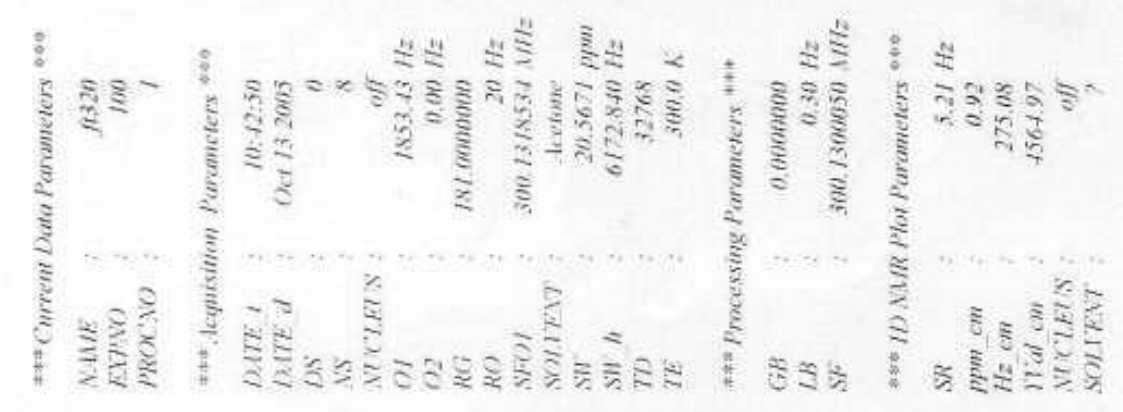

lito 2

009067

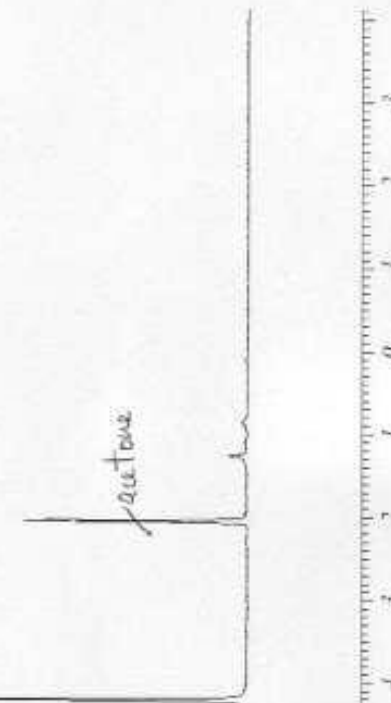

ENo2'

(1)

o

ह

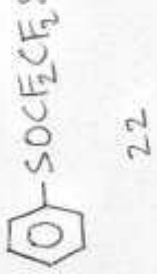



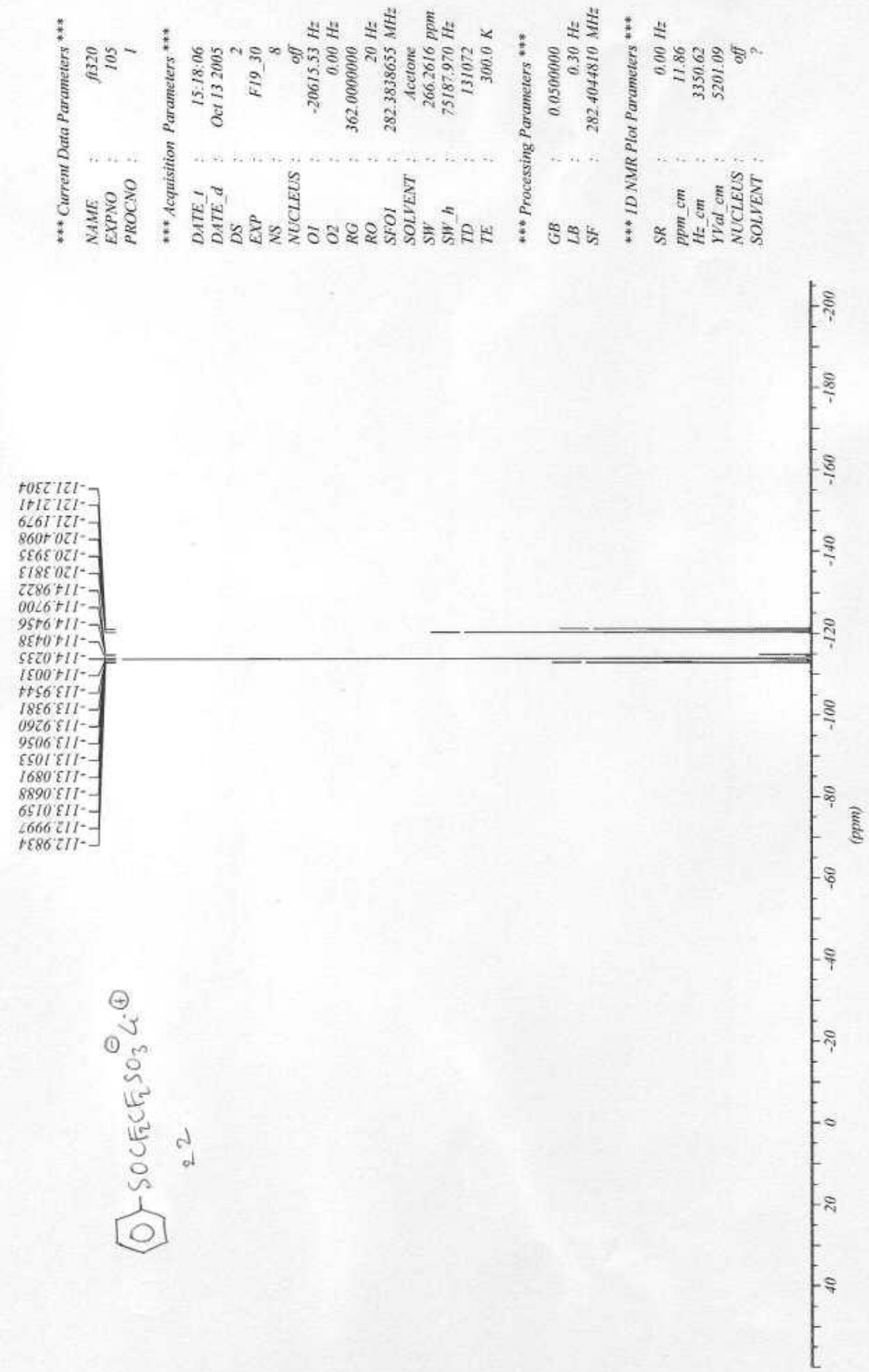

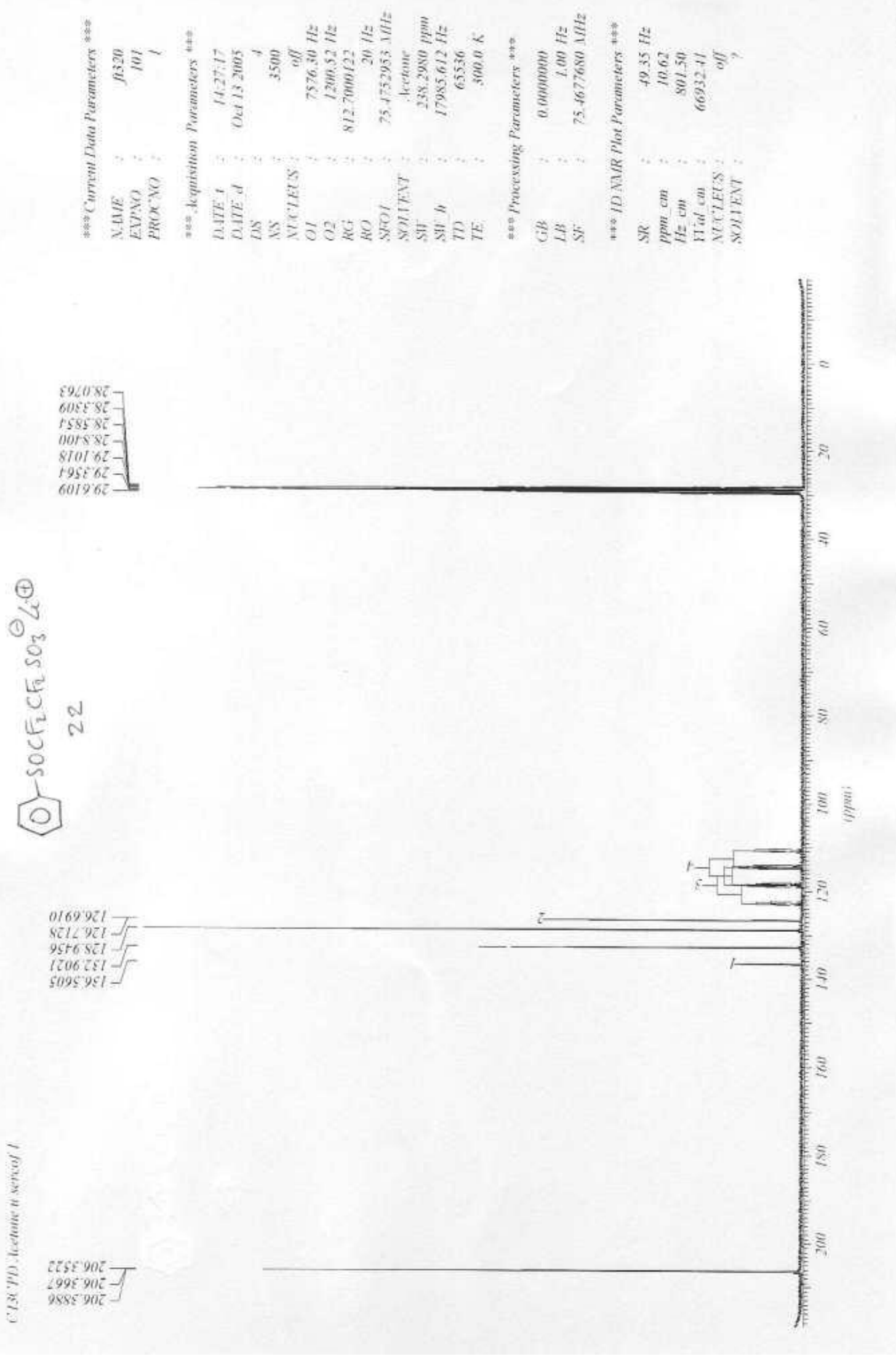

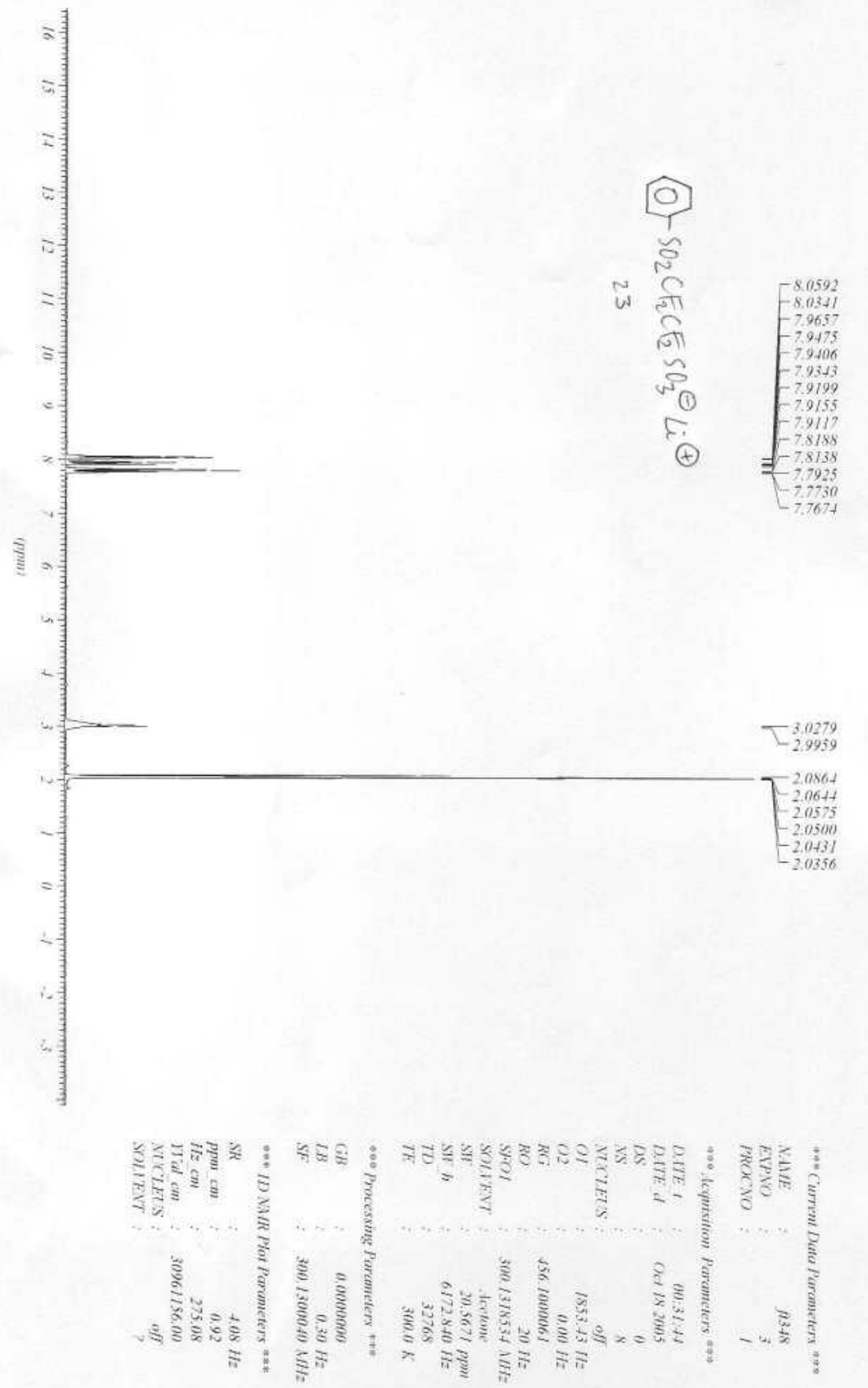

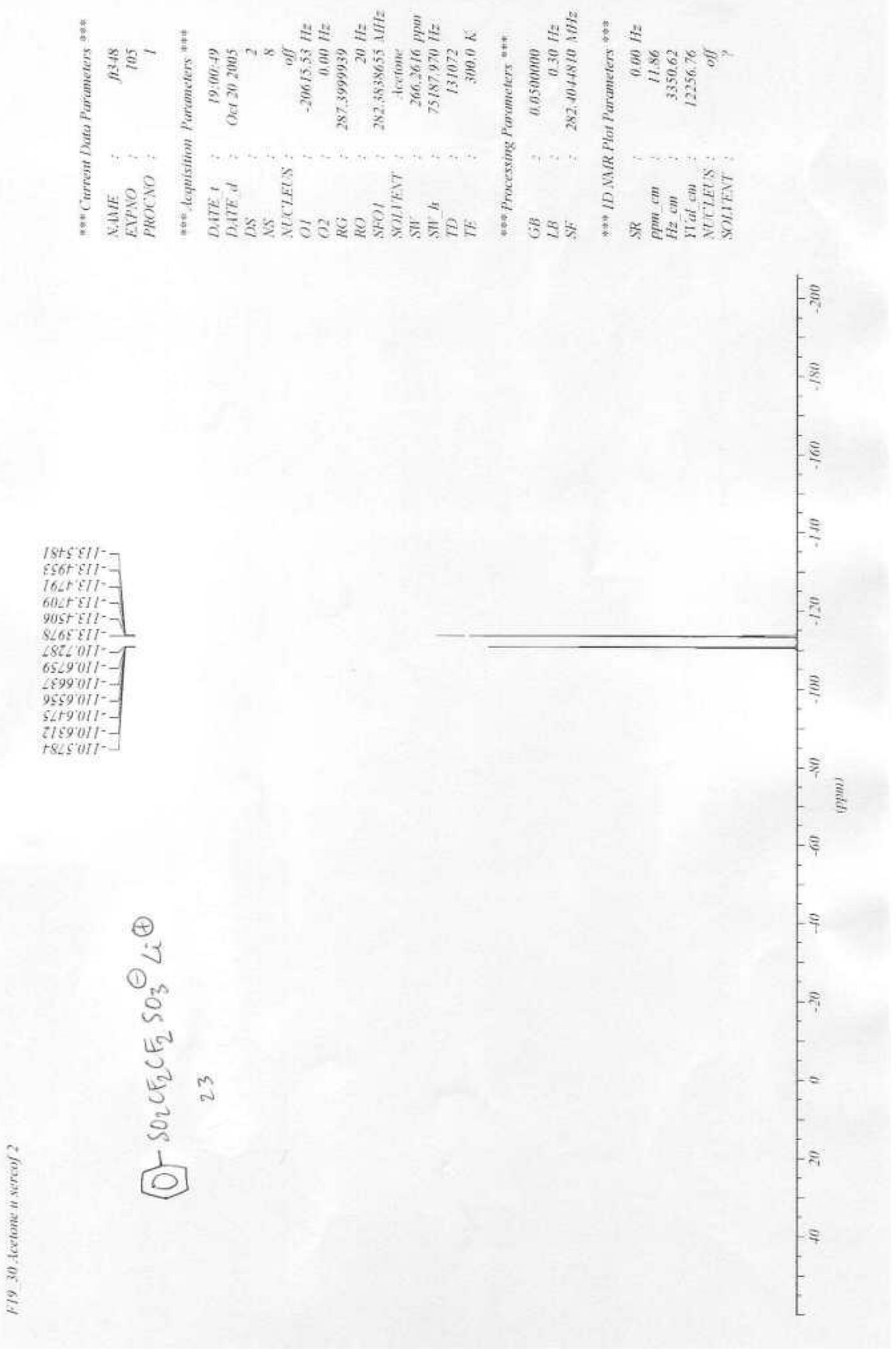

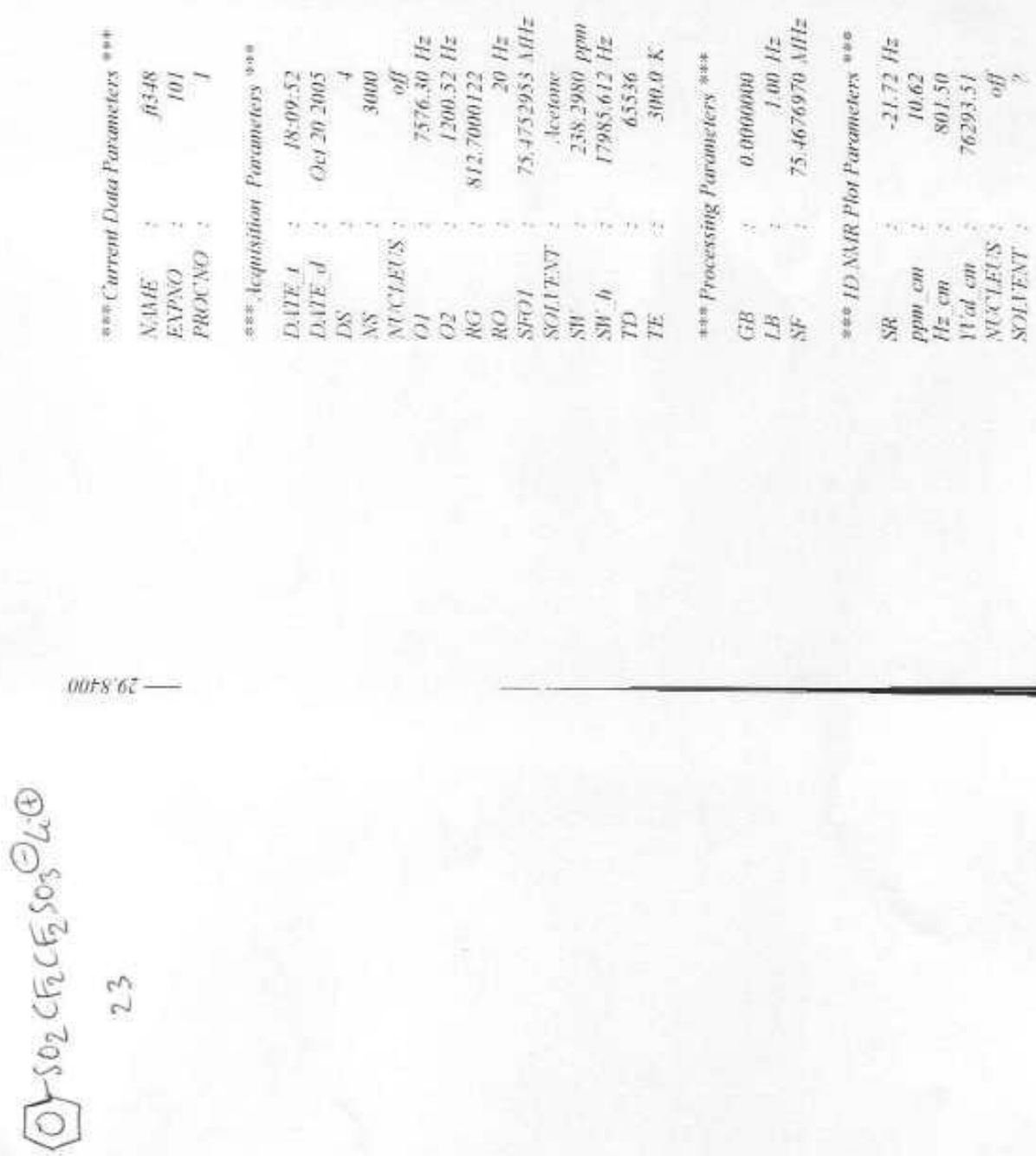

0865057

torel 5

It+Citil-

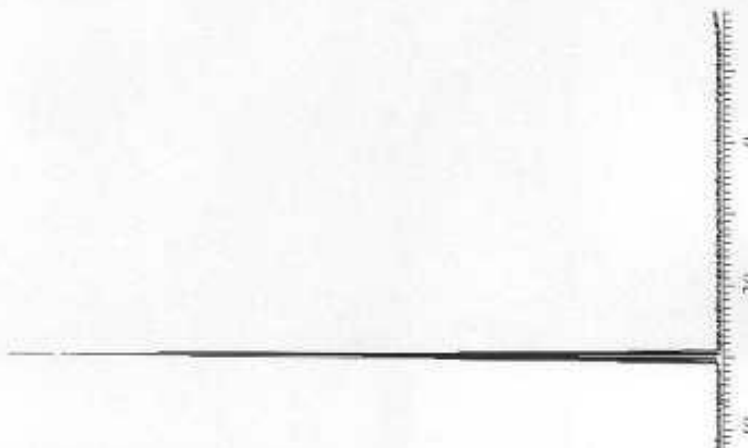

5

6928:206 

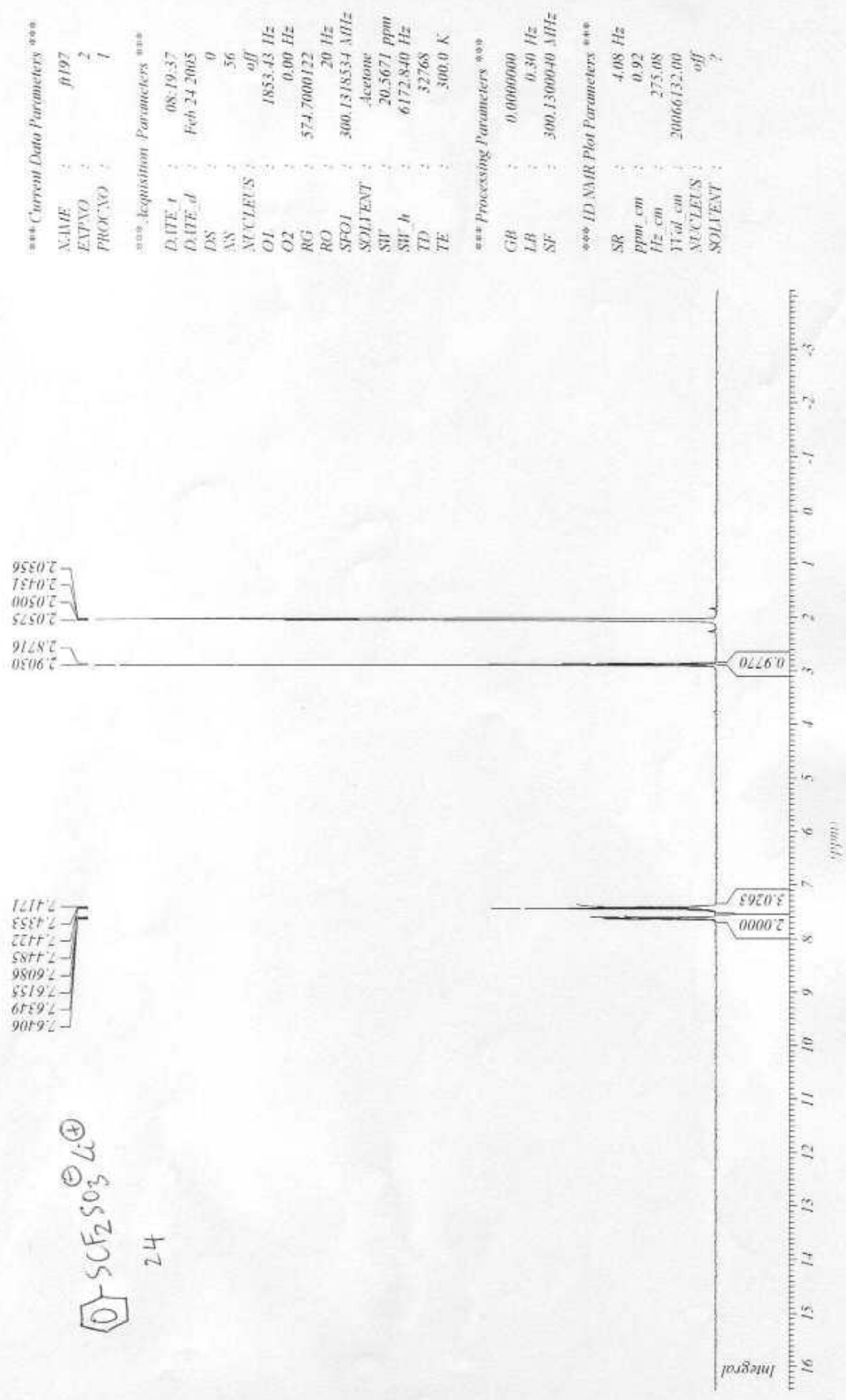


$$
7
$$




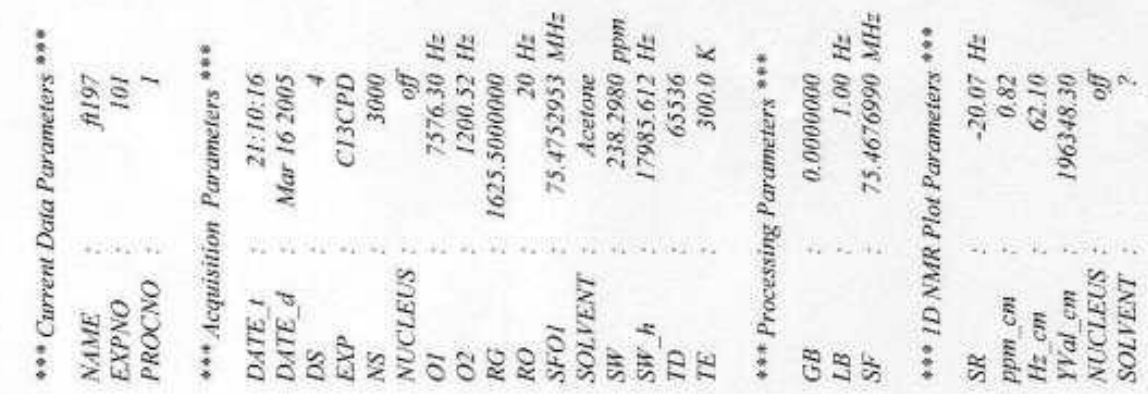

$9+6 \bar{z}+z l-$

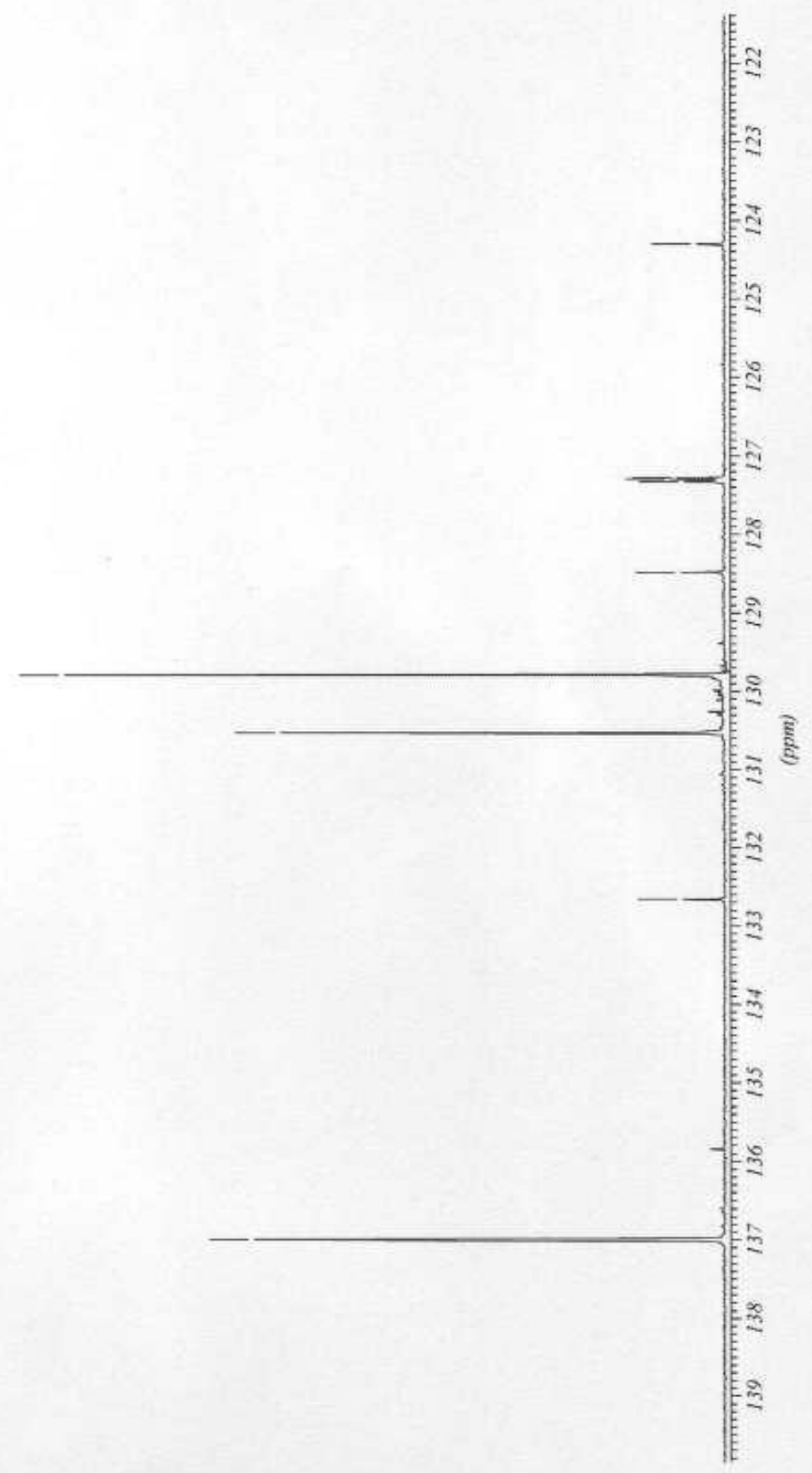



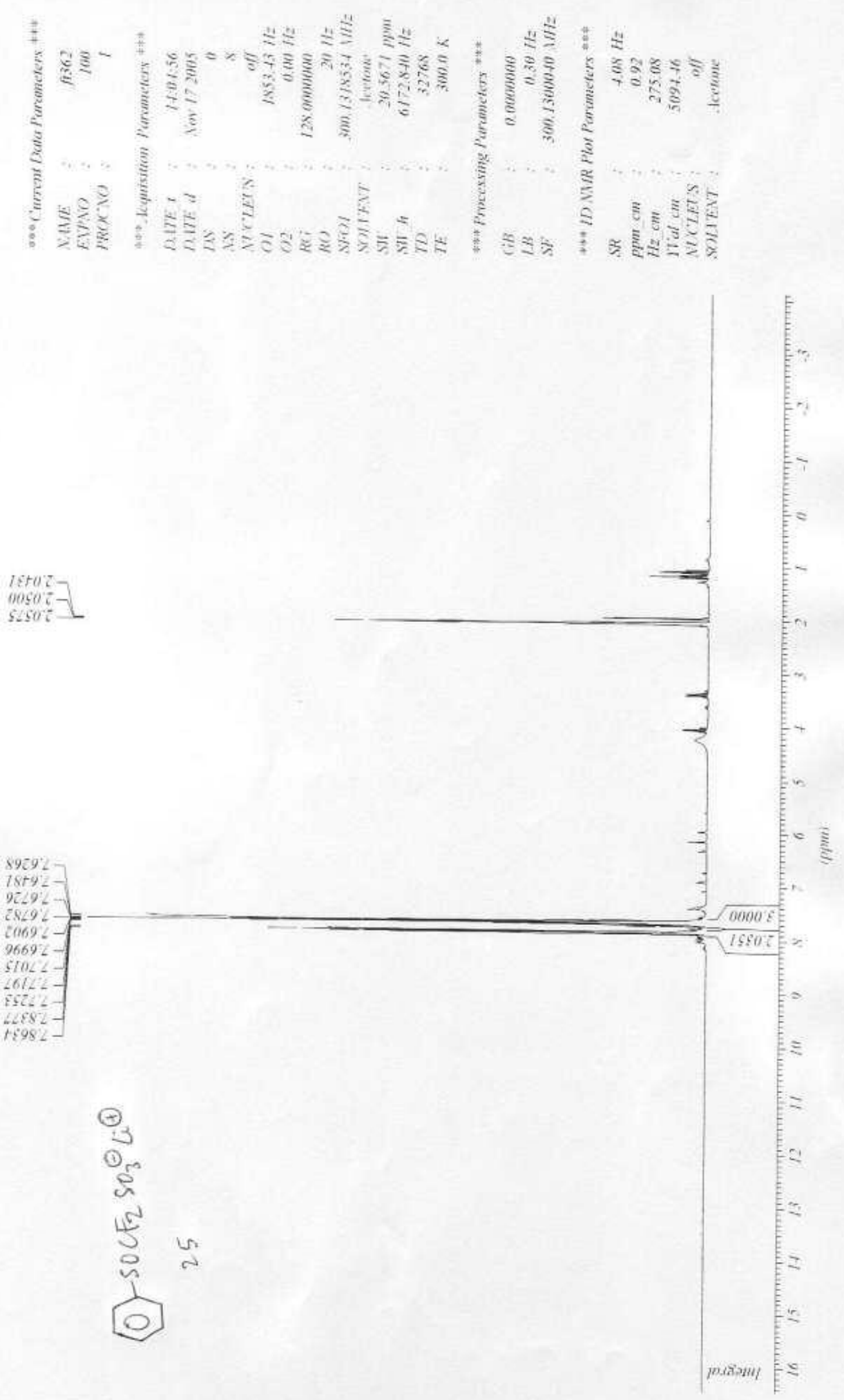


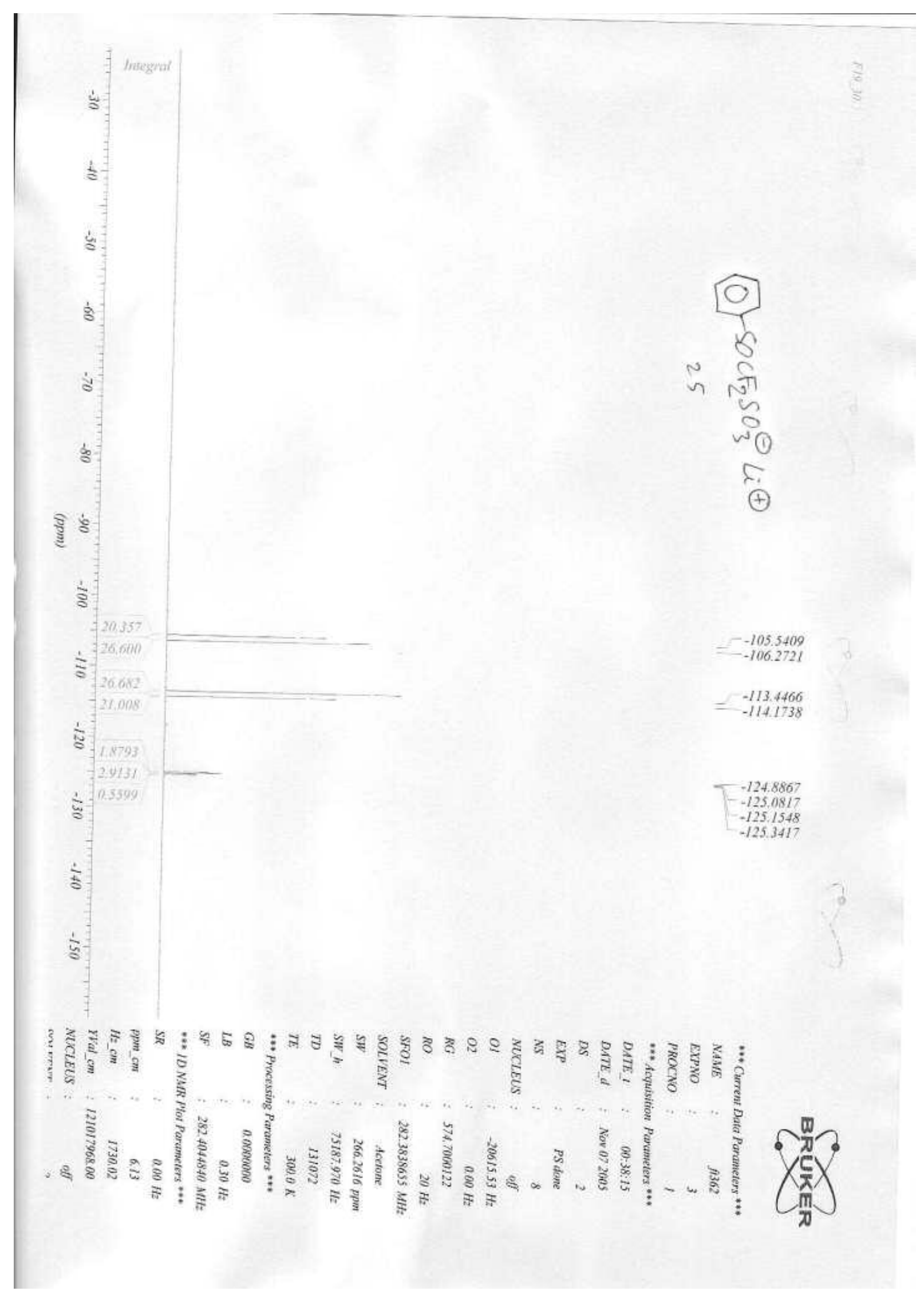




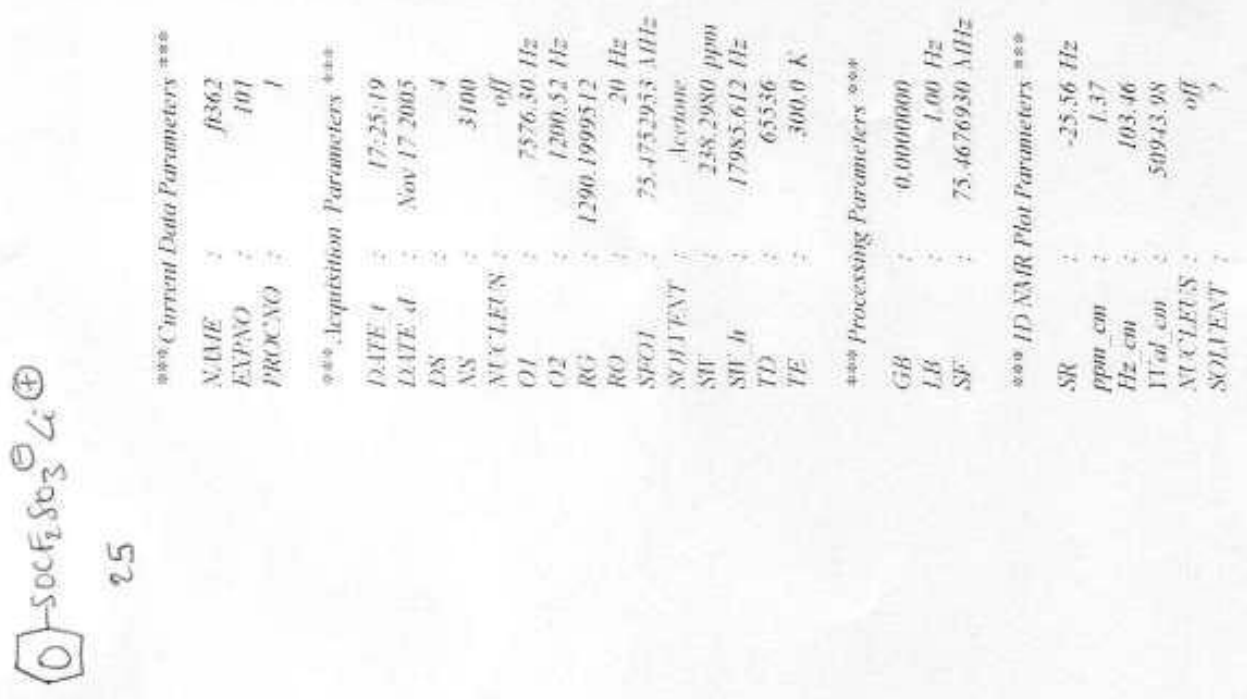

gIgt $911-$

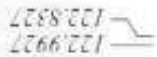

$889: 221-$

atos $2 t]$

5915221 드

$+686607$

$5920021=$

Eloxert-

HCSPE

$8209981-$

$9629.951-$ 

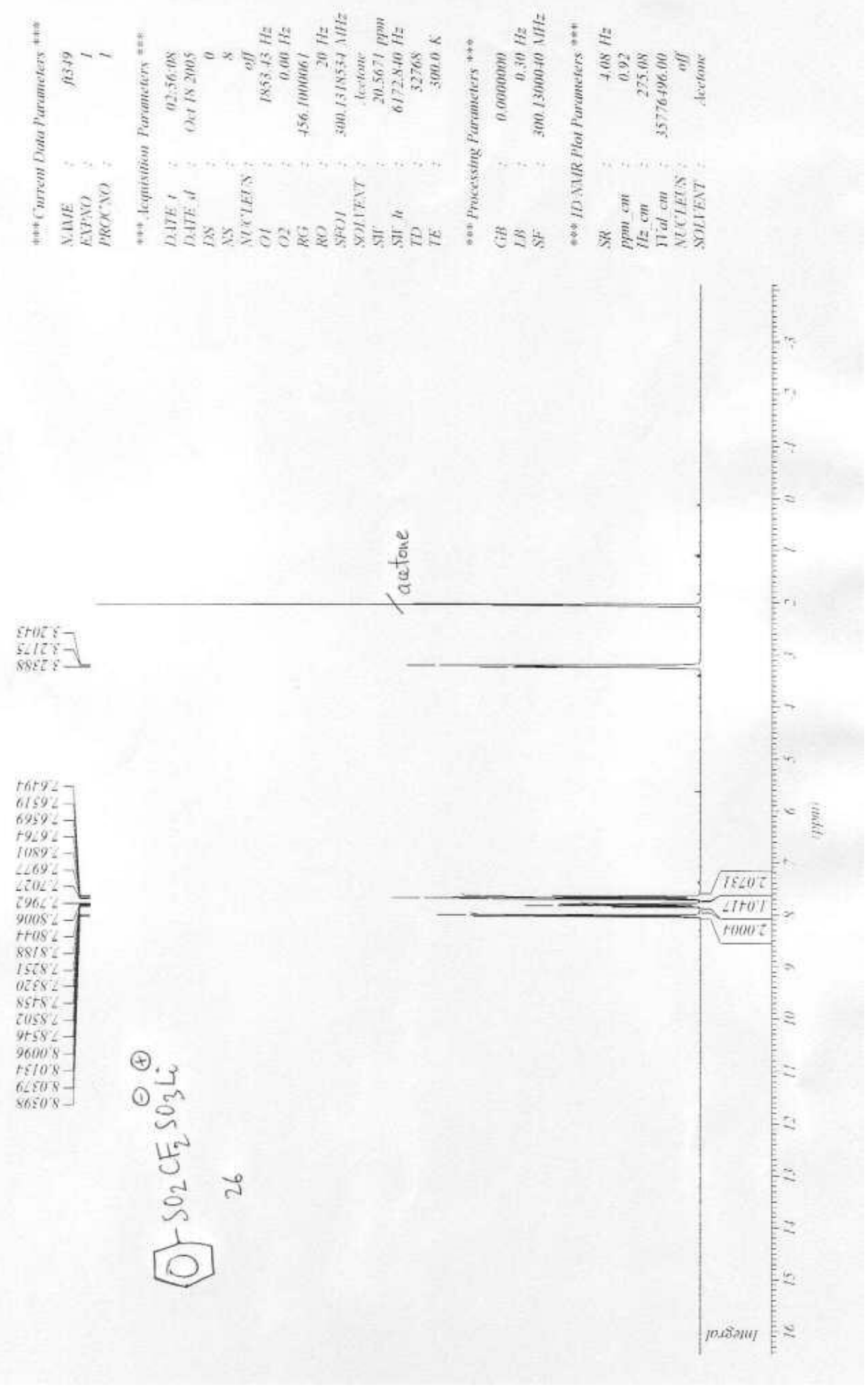

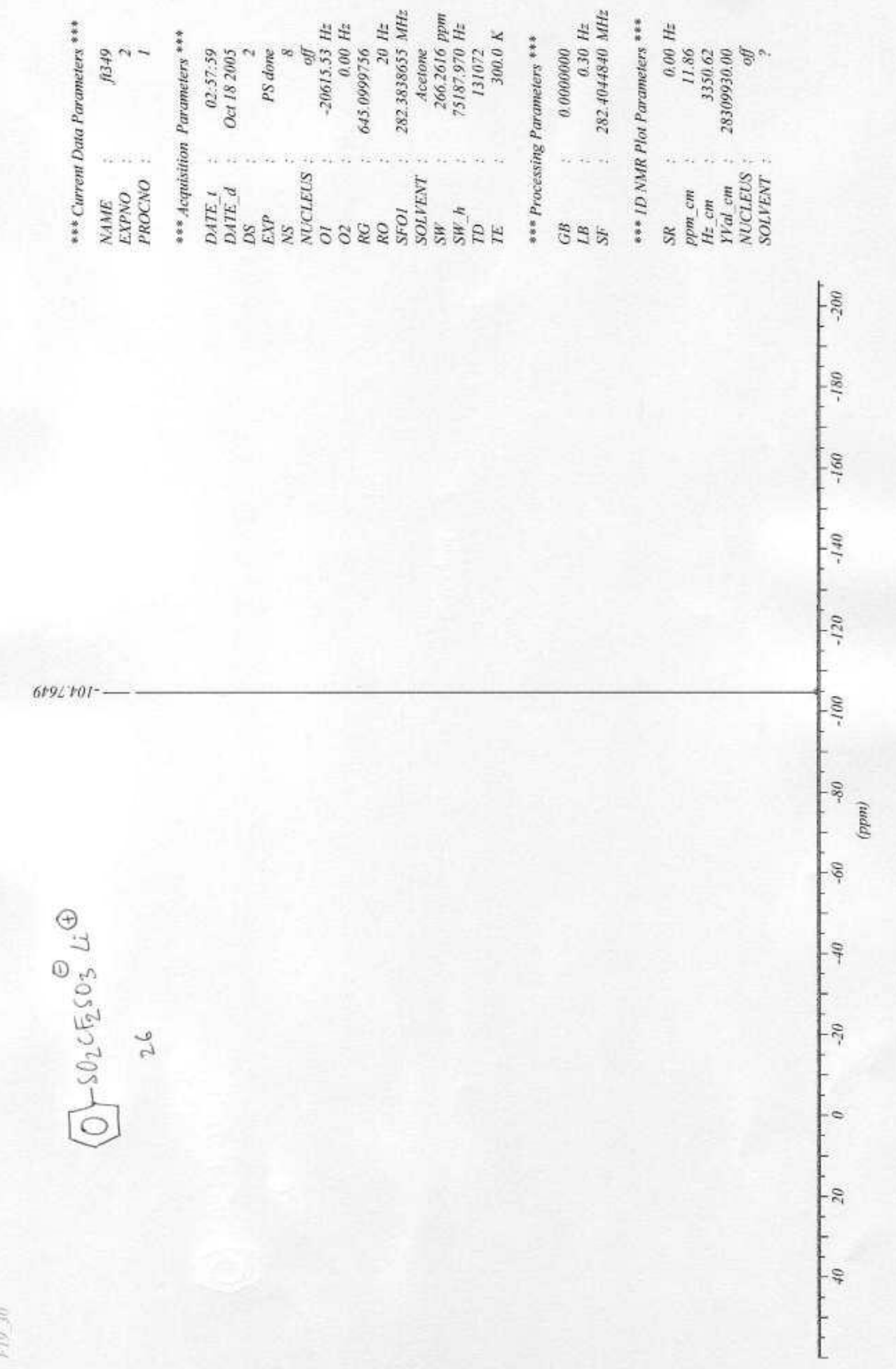

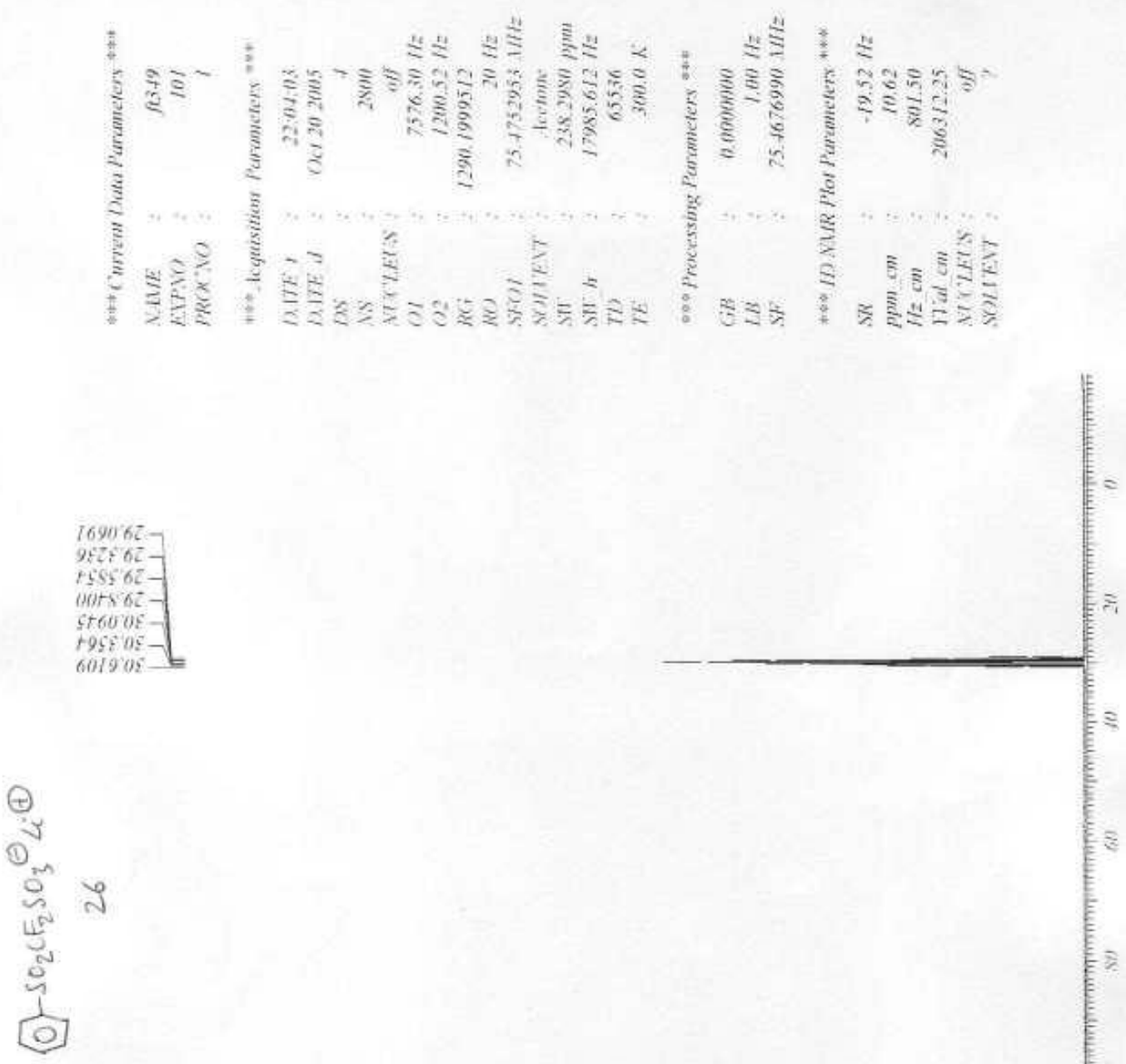

S885 $511-$

1255611

$226]+2]-$

$59 \mathrm{~s} 6 \mathrm{al}$

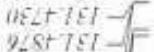

IT05 $\mid \mathrm{El}-$

If $065 s$ -

15: ges]

$289520 z-7$

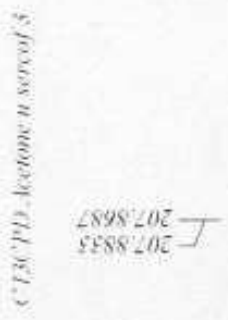



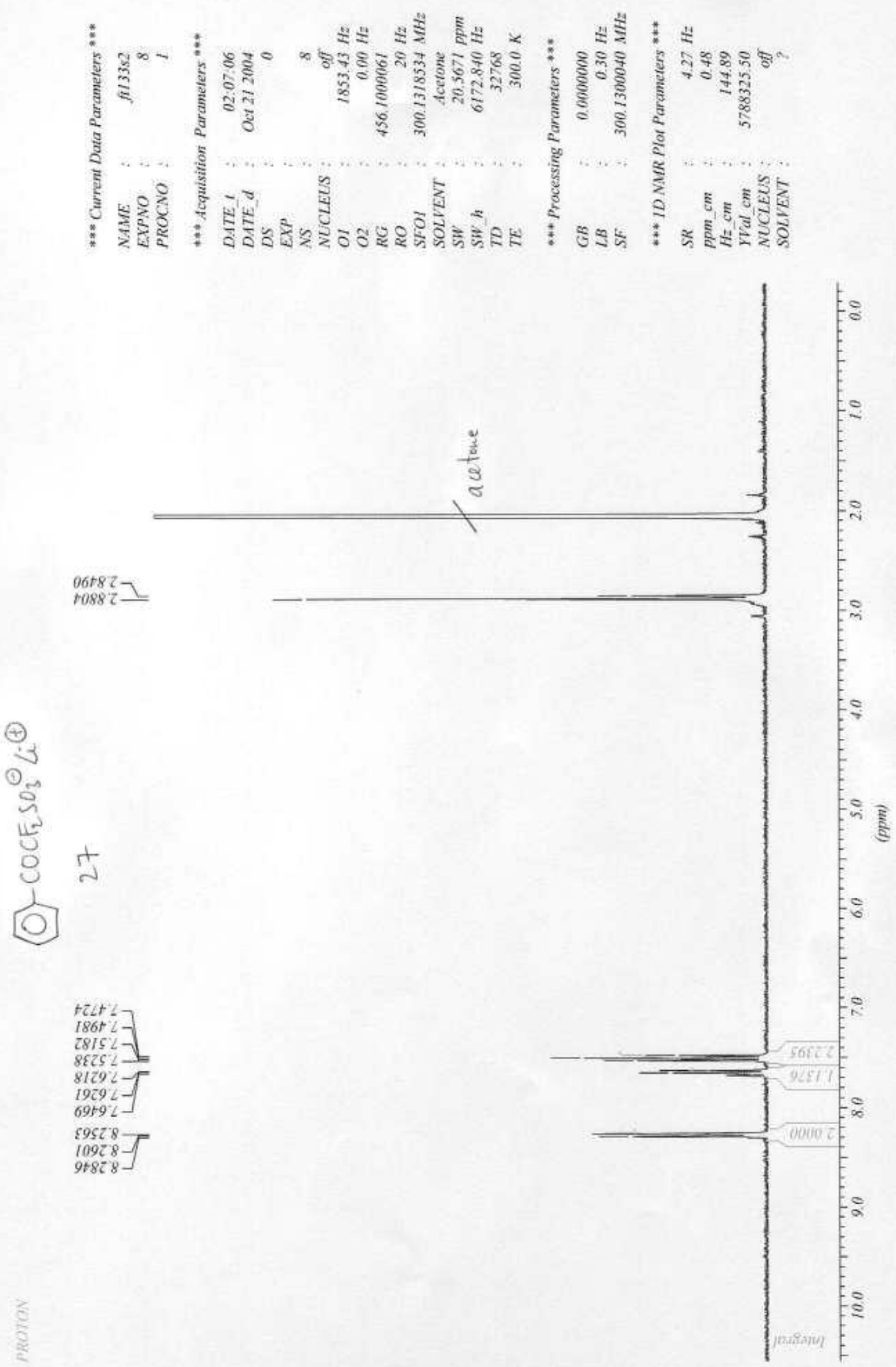
缙

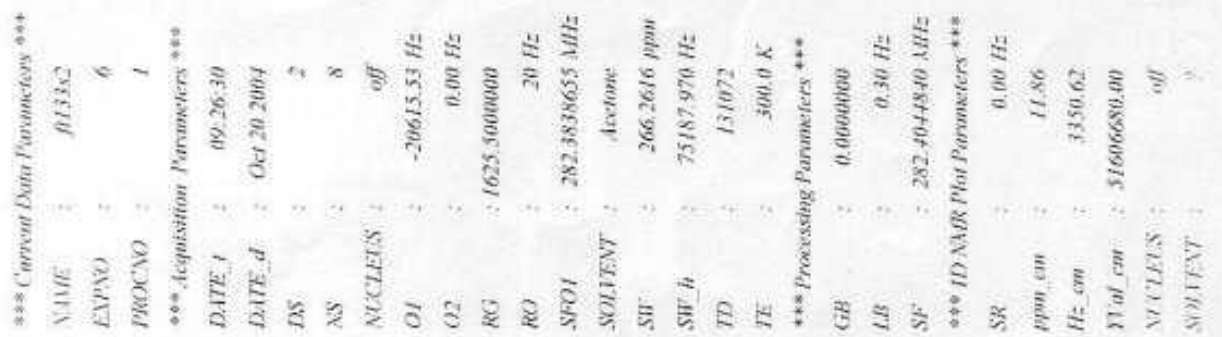

(†)

$0^{3}$

$y^{2} \pi$
3
1
0 


$$
\text { 嚾 }
$$

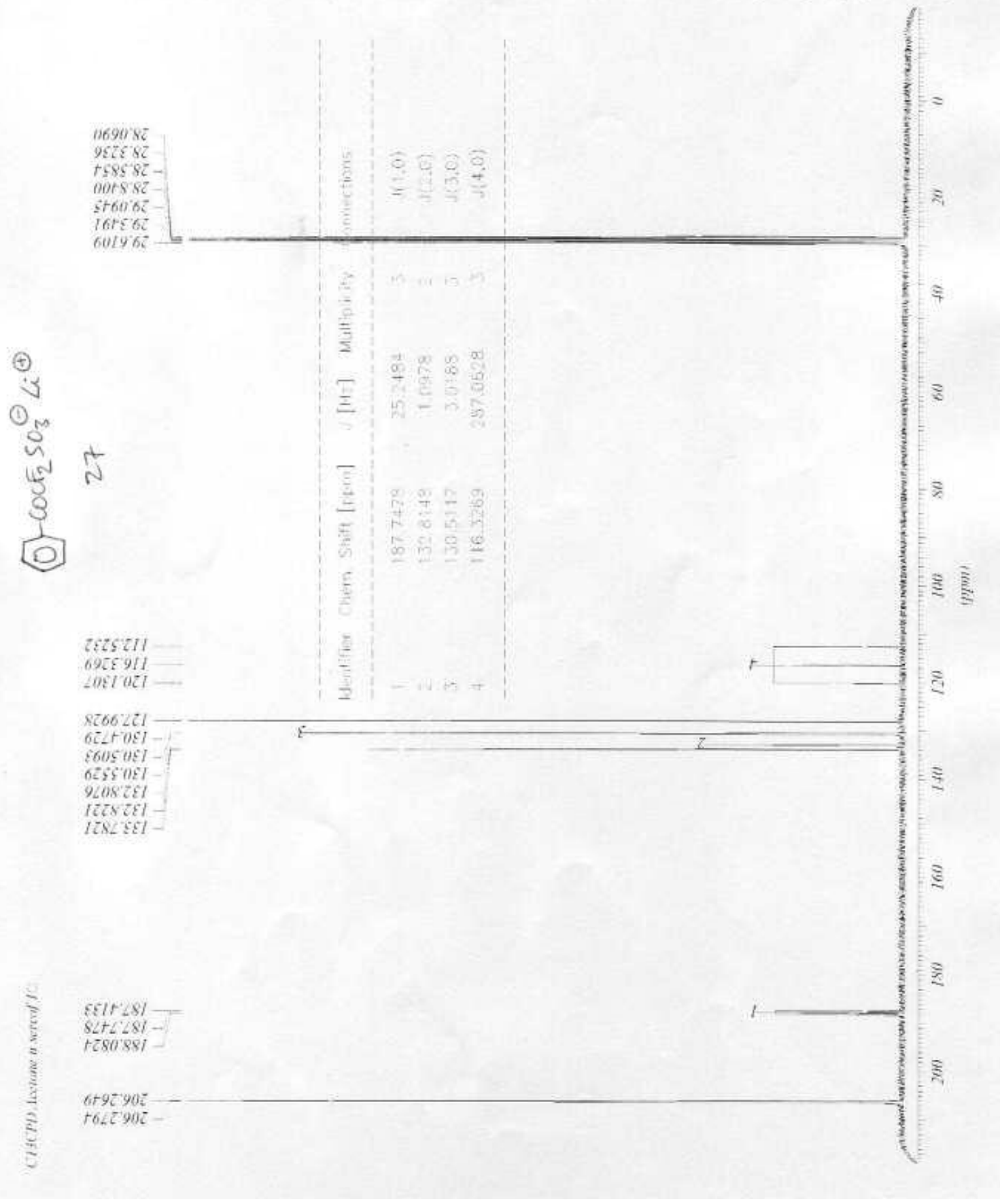

